Reduktionssysteme zur Berechnung einer Auflösung der orthogonalen freien Quantengruppen $\mathcal{A}_{o}(n)$

\author{
Dissertation \\ zur Erlangung des mathematisch-naturwissenschaftlichen Doktorgrades \\ „Doctor rerum naturalium“ \\ der Georg-August-Universität Göttingen \\ vorgelegt von \\ Johannes Härtel \\ aus Frankfurt am Main
}

Göttingen 2008 
Referent: Prof. Dr. Thomas Schick Korreferent: Prof. Dr. Andreas Thom Tag der mündlichen Prüfung: 


\section{Vorwort}

Schon Mitte der sechziger Jahre des 20. Jahrhunderts hatte Kac [Kac63] eine neue Klasse (,Ringgruppen“) von mathematischen Objekten zur Analyse der Pontryagindualität eingeführt, die später unter dem Begriff „Quantengruppen“ klassifiziert wurde.

Etwa zwanzig Jahre später beschäftigte sich Woronowicz [Wor87] [Wor88] mit „Pseudogruppen“, als er eine wichtige Familie von Matrixquantengruppen einführte. Sie lieferte neuartige Deformationen der zuvor noch als rigide eingestuften Liegruppe $S U(2)$. Diese Quanten- $S U(2)$-Gruppen lieferten wichtige Beispiele für die Quantenphysik, in der $S U(2)$ beispielsweise als Isospingruppe in der schwachen Wechselwirkung auftritt.

Woronowicz erklärte dabei die Quanten- $S U(2)$-Gruppen über die Charaktere einer axiomatisch vorgegebenen Hopfalgebra. Entsprechend stellen die Quanten- $S U(2)$ Gruppen Beispiele für Räume in der nicht-kommutativen Geometrie von Connes [Con85] dar. Diese Konstruktionen entsprechen denen von Drinfeld [Dri86] und Jimbo [Jim85] (vgl. [Ros87]).

Später, im Jahr 1995, definierten Wang [Wan95] und Van Daele [VDW96] die orthogonalen und die unitären freien Quantengruppen. Diese stellten sich als universell heraus, in dem Sinn, dass jede kompakte Matrixquantengruppe isomorph ist zu einem direkten Produkt von Unteralgebren der freien Quantengruppe. Dieses Paper zeichnet sich durch einen sehr konkreten, direkten Zugang zu Quantengruppen aus. Meine Arbeit bezieht sich auf Wangs Beschreibung von freien Quantengruppen.

Entsprechend der Universalität von Wang und Van Daeles Konstruktion ist die Homologie der beiden freien Quantengruppen von besonderem Interesse. Unter der Voraussetzung, dass eine bestimmte Sequenz exakt ist, haben Collins und Thom in [TC06] Schlüsse über die Homologien der orthogonalen und der unitären freien Quantengruppen gezogen, mit dem Ziel, ihre $\ell^{2}$-Bettizahlen zu bestimmen. Eine Übersicht zu orthogonalen Quantengruppen findet sich bei Collins und Banica [ $[\mathrm{BC} 07 \mathrm{a}]$.

Das wesentliche Problem, auf das Collins und Thom in ihrer Arbeit stoßen, ist, dass sie zwar explizit Erzeuger und Relationen der freien Quantengruppe, jedoch keine Basis kennen. In dieser Arbeit wird der Formalismus von Reduktionssystemen genutzt, um exemplarisch eine solche Basis zu berechnen und die Exaktheit von Collins und Thoms projektiver Auflösung zu beweisen.

Reduktionssysteme (synonym Gröbnerbasen oder Standardbasen) wurden 1964 und 1965 von Buchberger [Buc06], Hironaka [Hir64] sowie Knuth und Bendix [KB70] unabhängig voneinander beschrieben. 1978 zeigte Bergman [Ber78a], dass auch Gröbnerbasen bezüglich Moduln berechnet werden können.

Reduktionssysteme entwickelten sich zu einem wichtigen Werkzeug in der theoreti- 
schen Informatik, für Theorembeweise und funktionale Programmierung. Es gibt inzwischen sehr gute Software zur Berechnung von Reduktionssystemen im Kontext kommutativer Algebren (z.B. SINGULAR [GPS05]), und auch in der Literatur werden vornehmlich kommutative Algebren behandelt. Auch für nicht-kommutative Algebren gibt es Software (z.B. PLURAL [GLS03], MAGMA [BCP97], GAP [GAP06]), allerdings beschränkt diese sich auf Spezialfälle. Zur Berechnung werden Vervollständigungsalgorithmen (siehe [Buc76] und [KB70]) benutzt. Die mir bekannten Implementierungen reduzieren in jedem Schritt alle bekannten Regeln vollständig und nutzen Symmetrien nicht aus, daher terminieren sie für die freien Quantengruppen nur für $n<10$.

In Kapitel 1 trage ich die Ergebnisse für nicht-kommutative Algebren zusammen und formuliere sie direkt unter Verwendung des Diamantenlemmas. Insbesondere wird gezeigt, dass sich die Verifizierung eines Reduktionssystems für den Kern eines Modulhomomorphismus vereinfachen lässt, falls für eine Algebra ein Reduktionssystem bekannt ist.

Im zweiten Kapitel wird ein vollständiges Reduktionssystem bezüglich der freien orthogonalen Quantengruppen an und verifiziert. Für den Fall $n=2$ sind es nur sehr wenige Regeln und es kann ein endlicher Automat angegeben werden, der sämtliche Basiselemente darstellt:

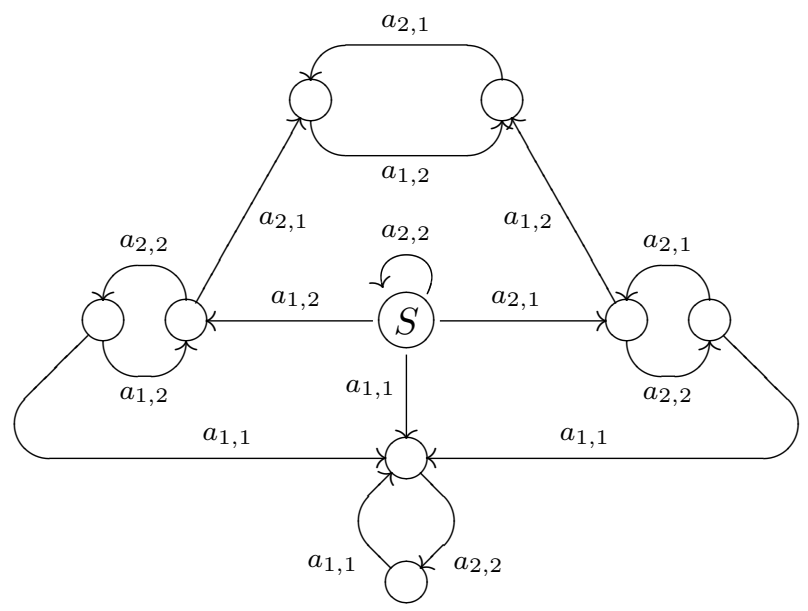

In Kapitel 3 werden die Ergebnisse aus Kapitel 1 und 2 genutzt, um eine Basis für die Kerne einer freien Auflösung der freien Quantengruppe als Bimodul zu beweisen.

Insbesondere im Fall von Collins und Thoms Sequenz liefert diese Methode die korrekten Kerne.

Abschließend wird im letzten Kapitel mit der nun verifizierten Auflösung die Homologie der orthogonalen freien Quantengruppen explizit berechnet.

Bedanken möchte ich mich beim Graduiertenkolleg „Gruppen und Geometrie“ für die finanzielle Unterstützung meiner Promotion. Anselm Knebusch danke ich für seine Bereitschaft, bei meinen Rechnungen Hilfestellung zu leisten. Besonders möchte ich mich bei Andreas Lochmann für seine Unterstützung mit seinem Wissen zu Ordnungen bedanken. Ivan Yudin schulde ich tiefen Dank für seinen Beistand während des gesamten Zeitraumes und seine hilfreichen Ratschläge. 


\section{Inhaltsverzeichnis}

\begin{tabular}{llr}
\hline 1 & Reduktionssysteme & 9
\end{tabular}

1.1 Abstrakte Reduktionssysteme . . . . . . . . . . . . . . . . . . . . 10

1.1 .1 Definitionen . . . . . . . . . . . . . . . . . 10

1.1 .2 Konfluenz . . . . . . . . . . . . . . . . . . . . 11

1.1 .3 Diamantenlemma . . . . . . . . . . . . . . . . . 15

1.2 Reduktionssystem bezüglich einer Algebra . . . . . . . . . . . . . . 18

1.2 .1 Definitionen . . . . . . . . . . . . . . . . 18

1.2 .2 Konfluenz . . . . . . . . . . . . . . . . . . . . . . . 22

1.2 .3 Überschneidungen . . . . . . . . . . . . . . . . . . . 23

1.2 .4 Ordnungen $\ldots \ldots \ldots \ldots \ldots$. . . . . . . . . . . . . . . . . . . . . . . . . . .

1.2 .5 Konstruktion von $r \ldots \ldots \ldots \ldots \ldots$

1.2 .6 Noethersch . . . . . . . . . . . . . . . . . . 33

1.3 Reduktionssystem bezüglich eines Moduls . . . . . . . . . . . . . . 34

$1.3 .1 \quad \mathcal{P}$-Vollständigkeit $\ldots \ldots \ldots \ldots \ldots \ldots$

$1.3 .2 \quad \boldsymbol{A} \otimes \boldsymbol{A}^{o p}$-Moduln $\ldots \ldots \ldots \ldots . \ldots \ldots$

$1.3 .3 \quad$ Kern eines $\boldsymbol{A} \otimes \boldsymbol{A}^{\text {op }}$-Homomorphismus . . . . . . . . . . . 43

\begin{tabular}{|lll}
2 & Konvergentes Reduktionssystem für $\mathcal{A}(n)$ & $\mathbf{5 1}$
\end{tabular}

$2.1 \quad$ Definition $\mathcal{A}(n) \quad \ldots \ldots \ldots \ldots \ldots \ldots \ldots \ldots$

2.2 Behebung der minimalen Überschneidungen . . . . . . . . . . . . 54

2.2 .1 Rechenregeln . . . . . . . . . . . . . . . 55

$2.2 .2 \quad$ Reduktionswege für $a_{p, 1} a_{q, 1} a_{y, 1} \ldots \ldots \ldots \ldots$. . . . . . . 59

2.2 .3 Reduktionswege für $a_{1, p} a_{1, q} a_{1, y} \ldots \ldots \ldots \ldots \ldots$. . . . . 60

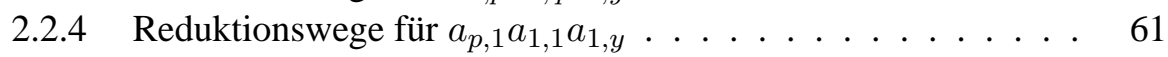

$2.2 .5 \quad$ Reduktionswege für $a_{1, p} a_{1,1} a_{y, 1} \ldots \ldots \ldots \ldots \ldots \ldots$

2.2.6 Reduktionswege für $a_{1,1} a_{1,1} \ldots \ldots \ldots \ldots \ldots \ldots$

2.2.7 Reduktionswege für $a_{p, 1} a_{q, 1} a_{y, 2} a_{z, 2} \ldots \ldots \ldots \ldots \ldots$

2.2 .8 Reduktionswege für $a_{1, p} a_{1, q} a_{2, y} a_{2, z} \ldots \ldots \ldots \ldots$. . . . 65

2.2.9 Reduktionswege für $a_{p, 1} a_{1,1} a_{2, y} a_{2, z} \ldots \ldots \ldots \ldots$

2.2 .10 Reduktionswege für $a_{1, p} a_{1,1} a_{y, 2} a_{z, 2} \ldots \ldots \ldots \ldots \ldots$

2.2.11 Reduktionswege für $a_{1,1} a_{2,1} a_{2, z} \ldots \ldots \ldots \ldots$. . . . . 70

2.2.12 Reduktionswege für $a_{1,1} a_{1,2} a_{z, 2} \ldots \ldots \ldots \ldots \ldots$. . . . . 71

2.2 .13 Reduktionswege für $a_{p, 1} a_{q, 2} a_{1,2} a_{1, y} \ldots \ldots \ldots \ldots \ldots$. . . . . . . . . . . .

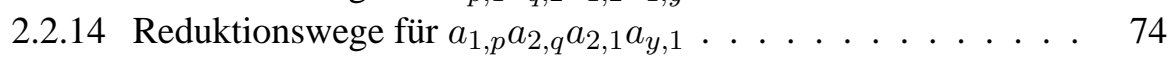

2.2 .15 Reduktionswege für $a_{p, 1} a_{1,2} a_{1,2} \ldots \ldots \ldots \ldots \ldots \ldots$

2.2.16 Reduktionswege für $a_{1, p} a_{2,1} a_{2,1} \ldots \ldots \ldots \ldots \ldots$ 
2.2.17 Reduktionswege für $a_{p, 1} a_{q, 2} a_{1,2} a_{2, y} a_{2, z} \ldots \ldots \ldots \ldots 78$

2.2 .18 Reduktionswege für $a_{1, p} a_{2, q} a_{2,1} a_{y, 2} a_{z, 2} \ldots \ldots \ldots \ldots$

2.2.19 Reduktionswege für $a_{p, 1} a_{1,2} a_{2,2} a_{2, z} \ldots \ldots \ldots \ldots$. . . . 82

2.2.20 Reduktionswege für $a_{1, p} a_{2,1} a_{2,2} a_{z, 2} \ldots \ldots \ldots \ldots \ldots$. . . 84

2.2.21 Reduktionswege für $a_{1,1} a_{2,2} a_{2,2} \ldots \ldots \ldots \ldots \ldots$

2.3 Lineare Basis für $\mathcal{A}(2)] \ldots \ldots \ldots$. . . . . . . . . . 88

3 Projektive Auflösungen 93

3.1 Auflösung von $\mathcal{A}(n)$ als Bimodul . . . . . . . . . . . . . . . . . . . . 94

$9.2 \Phi_{1} \ldots \ldots \ldots \ldots \ldots \ldots$

3.2.1 Übersichtstabelle für $\Phi_{1} \ldots \ldots \ldots \ldots$. . . . . . . . . . . . . . 97

$3.2 .2 \quad r_{\operatorname{Kern}\left(\Phi_{1}\right)} \ldots \ldots \ldots \ldots \ldots \ldots \ldots \ldots$

$3.2 .3 \quad r_{f s} \ldots \ldots \ldots \ldots \ldots \ldots \ldots$

$3.2 .4 \quad$ Rechenregeln . . . . . . . . . . . . . . . . . . . . . . . . . . . . . . 99

$3.2 .5 \quad$ Reduktionswege für $a_{p, 1} a_{q, 1} e \ldots \ldots \ldots \ldots$

3.2.6 Reduktionswege für $a_{1, p} a_{1, q} e \ldots \ldots \ldots \ldots \ldots$

3.2.7 Reduktionswege für $a_{p, 1} a_{q, 2} a_{r, 2} e \ldots \ldots \ldots \ldots$

3.2.8 Reduktionswege für $a_{1, p} a_{2, q} a_{2, r} \boldsymbol{e} \ldots \ldots \ldots \ldots$. . . . 106

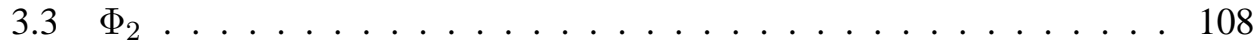

3.3.1 Übersichtstabelle für $\Phi_{2} \quad \ldots \ldots \ldots \ldots$. . . . . . . . . . . 108

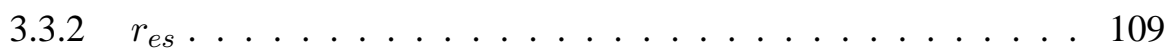

$3.3 .3 \quad r_{\operatorname{Kern}\left(\Phi_{2}\right)} \ldots \ldots \ldots \ldots \ldots \ldots \ldots$

3.3 .4 Rechenregeln . . . . . . . . . . . . . . . . . . . . . . . 110

$3.3 .5 \quad$ Reduktionswege für $a_{1, p} a_{1, n} e_{q, n} \ldots \ldots \ldots \ldots \ldots$

3.3.6 Reduktionswege für $a_{1, p} a_{2, q} a_{2, n} e_{r, n} \ldots \ldots \ldots \ldots \ldots$

3.3 .7 Reduktionswege für $a_{p, 1} a_{n, 1} \boldsymbol{e}_{n, q} \ldots \ldots \ldots \ldots \ldots \ldots$

3.3.8 Reduktionswege für $a_{p, 1} a_{q, 2} a_{n, 2} e_{n, r} \ldots \ldots \ldots \ldots \ldots$

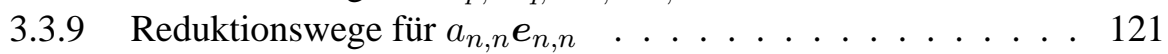

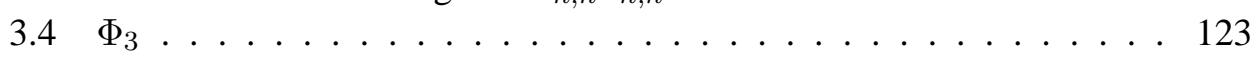

3.4 .1 Übersichtstabelle für $\Phi_{3} \ldots \ldots \ldots \ldots \ldots$. . . . . . . . . . . . . . . . . . . 123

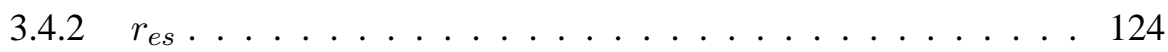

3.4 .3 Konflikte: . . . . . . . . . . . . . . . . . . . . . . . . . 124

3.4 .4 Reduktionswege für $a_{p, 1} a_{n, 1} \boldsymbol{f}_{n, n} a_{n, 1} \ldots \ldots \ldots \ldots$

3.4.5 Reduktionswege für $a_{n, 1} \boldsymbol{f}_{n, n} a_{n, 1} a_{q, 1} \ldots \ldots \ldots \ldots \ldots$

3.4.6 Reduktionswege für $a_{n, 1} \boldsymbol{f}_{n, n} a_{n, 1} a_{q, 2} a_{r, 2} \ldots \ldots \ldots \ldots$

3.4.7 Reduktionswege für $a_{p, 1} a_{n, 1} \boldsymbol{f}_{n, n} a_{n, 2} a_{q, 2} \ldots \ldots \ldots \ldots \ldots$

$3.4 .8 \quad$ Reduktionswege für $a_{p, 1} a_{q, 1} a_{n, 2} f_{n, n} a_{n, 2} \ldots \ldots \ldots \ldots \ldots$

3.4.9 Reduktionswege für $a_{1, p} a_{1,1} a_{n, 2} f_{n, n} a_{n, 2} \ldots \ldots \ldots \ldots$. . . 134

3.4.10 Reduktionswege für $a_{1, p} a_{2, q} a_{2,1} a_{n, 2} f_{n, n} a_{n, 2} \ldots \ldots \ldots \ldots$

3.4.11 Reduktionswege für $a_{n, 1} \boldsymbol{f}_{n, n} a_{n, 2} a_{1,2} a_{1, q} \mid \ldots \ldots \ldots$

3.4 .12 Reduktionswege für $a_{n, 1} \boldsymbol{f}_{n, n} a_{n, 2} a_{1,2} a_{2, q} a_{2, r} \ldots \ldots \ldots 144$

\begin{tabular}{|ccc}
\hline & (Ko)-Homologie & 149
\end{tabular}

4.0 .13 Spezialfall $n=1 \ldots \ldots \ldots \ldots$. . . . . . . . . . 150

4.0 .14 Auflösung für $n \geq 3 \ldots \ldots \ldots \ldots \ldots 151$

4.1 Tor . . . . . . . . . . . . . . . . . . . . . . 153

$4.1 .1 \Phi_{1 *}: \boldsymbol{E} \mapsto \Omega e-\Lambda \boldsymbol{e} \ldots \ldots \ldots \ldots \ldots 154$ 
$4.1 .2 \quad \Phi_{2 *}: \boldsymbol{F} \mapsto \Omega \boldsymbol{E}^{t}+\left(\Lambda \boldsymbol{E}^{t}\right)^{t} \ldots \ldots \ldots \ldots \ldots \ldots$

$4.1 .3 \quad 1$. Fall: $\mathbb{K}=\mathbb{R}$ und $n=2 \ldots \ldots \ldots \ldots$

$4.1 .4 \quad$ 2. Fall: $\Omega=\Lambda$, also $\Omega \Lambda^{t}=\mathrm{id} \ldots \ldots \ldots \ldots . \ldots 158$

4.1.5 3. Fall: $\Omega=-\Lambda$, also $\Omega \Lambda^{t}=-\mathrm{id} \ldots \ldots \ldots$. . . . . . 159

$4.1 .6 \quad$ Allgemeiner Fall $\ldots \ldots \ldots$. . . . . . . . . . . . . . . . . 160

$4.1 .7 \quad \Phi_{3 *}: \boldsymbol{f} \mapsto-\operatorname{tr}\left(\Lambda \Omega^{t} \boldsymbol{F}\right)+\operatorname{tr}(\boldsymbol{F}) \ldots \ldots . . \ldots . .164$

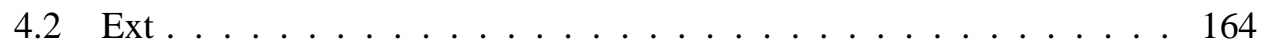

$4.2 .1 \quad \phi_{1}^{*}: \overline{\boldsymbol{e}} \mapsto(\Omega-\Lambda) \overline{\boldsymbol{E}} \ldots \ldots \ldots \ldots \ldots \ldots$

$4.2 .2 \quad \phi_{2}^{*}: \overline{\boldsymbol{E}} \mapsto\left(\Omega^{t} \overline{\boldsymbol{F}}\right)^{t}+\left(\Lambda^{t} \overline{\boldsymbol{F}}^{t}\right)^{t} \ldots \ldots \ldots \ldots \ldots \ldots$

$4.2 .3 \quad \phi_{3}^{*}: \overline{\boldsymbol{F}} \mapsto\left(\left(\Lambda \Omega^{t}\right)^{t}+\mathrm{id}\right) \overline{\boldsymbol{f}} \ldots \ldots \ldots \ldots \ldots \ldots$ 


\section{Kapitel 1}

\section{Reduktionssysteme}

Zum Rechnen in durch Gleichungen definierten Strukturen können Reduktionssysteme genutzt werden. Beispiele für solche Strukturen, die in dieser Arbeit betrachtet werden, sind Halbgruppen, Gruppen, Ringe, Algebren und Moduln. In den folgenden Kapiteln werden für Wörter über einem festen Alphabet Reduktionssysteme, man spricht auch von Wortersetzungssystemen oder Semi-Thue-Systemen, genutzt, um Gröbnerbasen zu Idealen zu berechnen.

Weitere Anwendungsbereiche für Reduktionssysteme, die hier nicht weiter betrachtet werden, sind Termersetzungssysteme für das Rechnen in logischen Strukturen. Sie finden Anwendung bei effizienten Verfahren zum automatischen Theorem-Beweisen in der Prädikatenlogik und zur Beschreibung von abstrakten Datentypen, insbesondere für Korrektheitsbeweise für Programme in einer hohen Programmiersprache.

Das Problem in durch Gleichungen definierten Strukturen ist, dass Gleichungen in beide Richtungen angewendet werden können und dass dadurch der Suchraum auch für eine Computer gestützte Berechnung zu groß wird. Der Trick bei Reduktionssystemen besteht darin die Gleichungen nur in eine Richtung anzuwenden. Der Suchraum wird dadurch stark eingeschränkt und man erhält eine mächtige Simplifikation.

Ein Beispiel dafür ist das Wortproblem. Sei $\mathcal{E}$ eine Menge und sei $\sim$ eine Äquivalenzrelation auf $\mathcal{E}$. Seien $v, w \in \mathcal{E}$. Die Frage, ob $v$ und $w$ äquivalent, also $v \sim w$, sind, heißt Wortproblem.

Das Problem ist schon für sehr einfache Gruppen nicht auf den ersten Blick lösbar.

Beispiel 1.0.1
Betrachten wir die Gruppe $G=<a, b \mid a b a, b^{2}>$. Gilt hier $a^{2} \sim b ?_{1}^{1}$

Um das Wortproblem zu lösen wählt man zunächst eine wohldefinierte Partialordnung ,>“ und Reduktionsrelationen $\mapsto$ auf $\mathcal{E}$, so dass gilt:

- Reduktion: Jede Relation verkleinert, also $\forall w_{1}, w_{2} \in \mathcal{E}$ mit $w_{1} \mapsto w_{2}$ gilt: $w_{1} \sim w_{2}$ und $w_{1}>w_{2}$.

\footnotetext{
${ }^{1} a b \sim a b a b a \sim b a$, also ist $b \sim b a b a \sim b b a a \sim a^{2}$.
} 
- Konvergenz: Zu jedem Element gibt es genau eine Normalform, also für jede Äquivalenzklasse $[w]$ existiert ein Element $\hat{w} \in[w]$, so dass es für jedes $w \in[w]$ eine Folge von Relationen gibt mit $w \mapsto \ldots \mapsto \hat{w}$.

Die Reduktionsrelationen erhält man, indem man die Äquivalenzrelationen durch ein Gleichungssystem beschreibt und dann die Relationen nimmt, die man durch Anwenden der Gleichungen von rechts nach links erhält. Dann startet man die Vervollständigung nach Knuth-Bendix ([KB70]). Dieser Algorithmus versucht zu einem gerichteten Gleichungssystem ein äquivalentes konfluentes Reduktionssystem zu finden.

Dieses aus drei Abschnitten bestehende Kapitel basiert auf [Ave95], [New42], [Gre03] und [Ber78b]. Zunächst werden abstrakte Reduktionssysteme vorgestellt, danach Ersetzungssysteme für Algebren und Moduln. Im letzten Abschnitt wird insbesondere eine effiziente Methode zur Berechnung des Kerns einer A-Modul Abbildung vorgestellt.

\subsection{Abstrakte Reduktionssysteme}

In diesem Abschnitt werden die für Reduktionssysteme nötigen Begriffe definiert und es wird ein Beweis für das Diamantenlemma angegeben.

Damit man leicht mit einem Reduktionssystem arbeiten kann, muss es die so genannte Church-Rosser Eigenschaft erfüllen. Der Nachweis dieser Eigenschaft ist sehr mühsam. Leichter ist Konfluenz und noch leichter lokale Konfluenz zu beweisen. Am Ende dieses Abschnittes werden wir mit dem Diamantenlemma zeigen, dass diese drei Eigenschaften unter gewissen Voraussetzungen gleich sind.

\subsubsection{Definitionen}

Zunächst definieren wir für eine beliebige Menge $\mathcal{E}$ ein Reduktionssystem $\mathcal{R}$.

\section{Definition 1.1.1 (Reduktionssystem)}

Eine Teilmenge $\mathcal{R} \subset \mathcal{E} \times \mathcal{E}$ heißt Reduktionssystem auf $\mathcal{E}$.

Ein Element $(x, y) \in \mathcal{R}$ heißt Reduktionsregel und wir schreiben dann:

$$
x \longrightarrow \underset{\mathcal{R}}{ } y .
$$

Wir sagen: „, $x$ wird zu $y$ reduziert“. Wenn eindeutig ist, welches Reduktionssystem gemeint ist, schreiben wir statt $x \longrightarrow{ }_{\mathcal{R}} y$ oft nur $x \longrightarrow y$.

Trotz der Schreibweise ist ein Reduktionssystem keine Abbildung zwischen Mengen. Es ist durchaus erlaubt, dass $x$ zu zwei verschiedenen $y_{1}, y_{2}$ reduziert werden kann. 


\section{Definition 1.1.2 (Unreduzierbar)}

Ein Element $z \in \mathcal{E}$ heißt unreduzierbar, falls es keine Reduktionsregel gibt, bei der $z$ auf der linken Seite steht, also:

$$
\forall w \in \mathcal{E} \text { gilt: }(z, w) \notin \mathcal{R} \text {. }
$$

Definition 1.1.3 (Reduktionsweg …..... $\overrightarrow{\mathcal{R}}_{\mathrm{R}}$ )

Ein Reduktionsweg ist eine Folge von Reduktionsregeln $\left(x_{i}, y_{i}\right)_{i \in I} \in \mathcal{R}$, so dass für alle $i \in I$ gilt:

$$
y_{i}=x_{i+1}
$$

Es gibt also einen Graphen:

$$
x_{1} \overrightarrow{\mathcal{R}}_{1} y_{1} \cdots \overrightarrow{\mathcal{R}}_{y_{n}} \mathrm{\longrightarrow}_{\mathcal{R}} \cdots
$$

Wir schreiben einen endlichen Reduktionsweg als $x_{1} \cdots>_{\mathcal{R}} y_{n}$. Wenn eindeutig ist, welches Reduktionssystem gemeint ist, schreiben wir statt $x \quad \cdots \ldots \ldots . \mathcal{R}_{\mathcal{R}} y$ oft nur $x \quad \cdots \cdots \cdots>y$. Ein Reduktionsweg kann leer, endlich oder unendlich sein.

$\mathrm{Zu}$ einem Element gibt es Reduktionswege, die von ihm ausgehen. Manche dieser Wege enden in einem unreduzierbaren Element. Dies motiviert folgende Definition.

\section{Definition 1.1.4 (Normalform)}

Eine Normalform eines Elements $x \in \mathcal{E}$ ist ein unreduzierbares Element $\mathcal{N}(x) \in \mathcal{E}$, so dass es einen endlichen (oder leeren) Reduktionsweg gibt, der in $x$ beginnt und in $\mathcal{N}(x)$ endet, also :

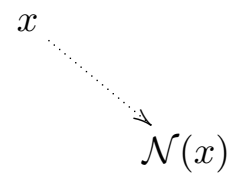

\section{Bemerkung 1.1.5 (Normalform)}

Eine Normalform ist im Allgemeinen nicht eindeutig. In einem abstrakten Reduktionssystem kann es für ein Element verschiedene Reduktionswege geben, die in verschiedenen unreduzierbaren Elementen enden.

\section{Definition 1.1.6 (Church-Rosser-äquivalent)}

Zwei Elemente $y_{1}, y_{n}$ heißen Church-Rosser-äquivalent bezüglich $\mathcal{R}$, falls sie über ungerichtete Reduktionsregeln aus $\mathcal{R}$ miteinander verbunden sind; es also eine endliche Folge $\left(y_{i}, y_{i+1}\right)_{i=1 \ldots n-1}$ gibt, wobei $\left(y_{i}, y_{i+1}\right) \in \mathcal{R}$ oder $\left(y_{i+1}, y_{i}\right) \in \mathcal{R}$.

Für zwei Church-Rosser-äquivalente Elemente $x, \bar{x}$ schreiben wir $x<{ }_{\mathcal{R}}>\bar{x}$.

\subsubsection{Konfluenz}

Ziel ist es möglichst einfach zu erkennen, ob die Normalformen eindeutig sind. Dazu definieren wir einige Eigenschaften für Reduktionssysteme. 


\section{Definition 1.1.7 (noethersch)}

Ein Reduktionssystem $\mathcal{R}$ heißt noethersch oder terminierend, wenn jeder Reduktionsweg endlich ist.

\section{Bemerkung 1.1.8 (Normalform)}

Falls $\mathcal{R}$ noethersch ist, dann hat jedes Element wenigstens eine Normalform.

\section{Definition 1.1.9 (lokal konfluent)}

Ein Reduktionssystem $\mathcal{R}$ heißt lokal konfluent, falls es für je zwei Reduktionsregeln $\left(x, y_{1}\right),\left(x, \bar{y}_{1}\right)$, die vom selben Element $x$ starten, zwei endliche Wege $\left(y_{i}, y_{i+1}\right)_{i=1 \ldots n}$, $\left(\bar{y}_{j}, \bar{y}_{j+1}\right)_{j=1 \ldots \bar{n}}$ gibt, die mit $y_{1}$ bzw. $\bar{y}_{1}$ beginnen und im selben Element $z$ enden, also zu jedem:
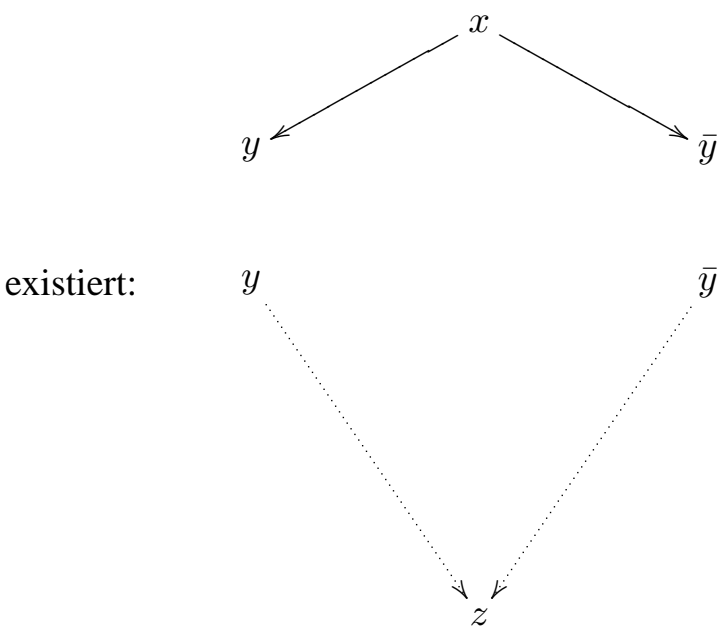

\section{Definition 1.1.10 (total konfluent)}

Ein Reduktionssystem $\mathcal{R}$ heißt total konfluent, falls es zu je zwei endlichen Wegen, die vom selben Element $x$ starten, zwei endliche Wege gibt, die diese Wege so fortsetzen, dass sie im selben Element $z$ enden, also zu jedem:
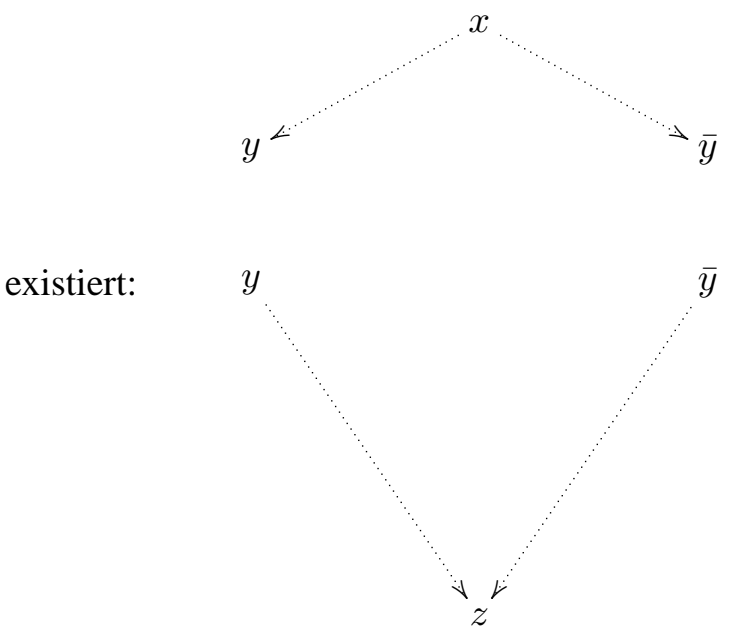

Definition 1.1.11 (Church-Rosser Eigenschaft)

Ein Reduktionssystem $\mathcal{R}$ erfüllt die Church-Rosser Eigenschaft, falls sich je zwei 
Church-Rosser-äquivalente Elemente $y, \bar{y}$ über jeweils nur absteigende Kanten zusammenführen lassen, also zu jedem:

$$
y<\mathcal{R}^{>} \bar{x}
$$

existiert: $\quad y \quad \bar{y}$

\section{Definition 1.1.12 (Konvergenz)}

Ein Reduktionssystem $\mathcal{R}$ heißt konvergent, wenn es noethersch ist und es zu jedem $x \in \mathcal{E}$ genau eine Normalform gibt.

In der Literatur findet man anstelle von Konvergenz auch die Begriffe kanonisch oder vollständig. Wir werden den Begriff „vollständig“ für einen Spezialfall von Konvergenz gebrauchen.

\section{Bemerkung 1.1.13 (totale Konfluenz $\neq$ lokale Konfluenz)}

Aus totaler Konfluenz folgt lokale Konfluenz, die Umkehrung gilt allerdings im Allgemeinen nicht. Dazu betrachten wir folgendes Gegenbeispiel:

\section{Beispiel 1.1.14}

Seien folgende Reduktionsregeln gegeben:

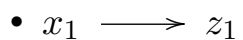

- $x_{1} \longrightarrow x_{2}$

- $x_{2} \longrightarrow x_{1}$

- $x_{2} \longrightarrow z_{2}$

Das zugehörige Reduktionssystem können wir darstellen durch:<smiles></smiles>

Von keinem Element aus gibt es zwei Reduktionsregeln, die nach $z_{1}$ bzw. $z_{2}$ reduzieren; alle anderen Paare von zwei Elementen können wir sogar auf die gleiche Normalform reduzieren. Also ist dieses Reduktionssystem lokal konfluent.

\begin{tabular}{|c|c|c|c|c|}
\hline & $x_{1}$ & $x_{2}$ & $z_{1}$ & $z_{2}$ \\
\hline$x_{1}$ & $\longrightarrow z_{1}$ & $\longrightarrow z_{1}$ & $\longrightarrow z_{1}$ & $\longrightarrow z_{2}$ \\
\hline$x_{2}$ & $\longrightarrow z_{1}$ & $\longrightarrow z_{1}$ & $\longrightarrow z_{1}$ & $\longrightarrow z_{2}$ \\
\hline$z_{1}$ & $\longrightarrow z_{1}$ & $\longrightarrow z_{1}$ & $\longrightarrow z_{1}$ & nicht reduzierbar \\
\hline$z_{2}$ & $\longrightarrow z_{1}$ & $\longrightarrow z_{1}$ & nicht reduzierbar & $\longrightarrow z_{2}$ \\
\hline
\end{tabular}


Die Elemente $z_{1}$ und $z_{2}$ sind unreduzierbar, aber es gibt in $x_{1}$ startende Reduktionswege, die nach $z_{1}$ und $z_{2}$ reduzieren. Also ist dieses Reduktionssystem nicht total konfluent.

Da es einen unendlich langen Redukionsweg gibt, nämlich

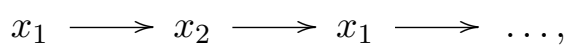

ist dieses Reduktionssystem nicht noethersch.

Lemma 1.1.15 (total konfluent $\Leftrightarrow$ Church-Rosser Eigenschaft)

Ein Reduktionssystem erfüllt genau dann die Church-Rosser Eigenschaft, wenn es total konfluent ist.

Beweis: [Lemma 1.1.15]

„Æ“:

Sei für ein Reduktionssystem die Church-Rosser Eigenschaft erfüllt, dann sind alle Paare, die über ungerichtete Regeln miteinander verbunden sind, über ausschließlich absteigende Kanten zusammenführbar. Also ist das Reduktionssystem total konfluent. , $\Rightarrow$ ":

Für die Umkehrung wollen wir zeigen, dass sich in einem total konfluenten Reduktionssystem, je zwei Elemente, die über ungerichtete Regeln miteinander verbunden sind, auf das gleiche Element reduzieren lassen. Für den Beweis nutzen wir eine vollständige Induktion:

Seien $x_{1}$ und $x_{2}$ über $n$ Church-Rosser-äquivalent.

- Induktionsbeginn $n=0$ : Für den Weg der Länge 0 gilt $x_{1}=x_{2}$.

- Induktionsannahme: Alle Elemente, die über $n$ ungerichtete Regeln miteinander verbunden sind, lassen sich auf das gleiche Element reduzieren.

- Induktionsschritt $n \Rightarrow n+1$ : Dann gibt es ein $y$ mit:
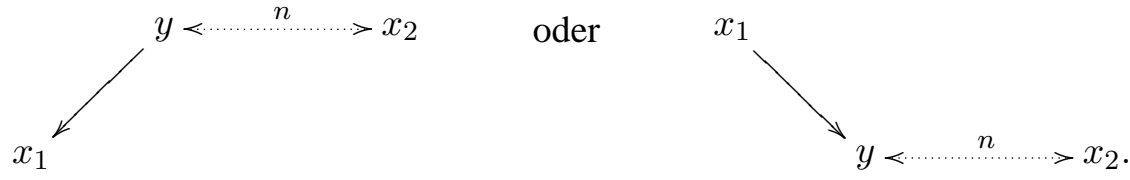

Nach Induktionsannahme lassen sich $y$ und $x_{2}$ über einen Weg $\quad->\mathrm{zu} z$ reduzieren.

Im ersten Fall gibt es wegen totaler Konfluenz zu $x_{1}$ und $z$ Wege $\ldots \ldots \cdots>>$, die sich in $z^{\prime}$ wieder zusammenführen lassen. Der Weg von $x_{2}$ nach $z$ lässt sich mit dem Weg $z$ nach $z^{\prime}$ fortsetzen.

Im zweiten Fall lässt sich der Weg von $x_{1}$ nach $y$ durch den Weg $y$ nach $z$ fortsetzen.

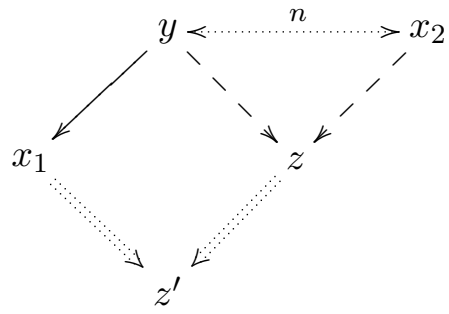

oder

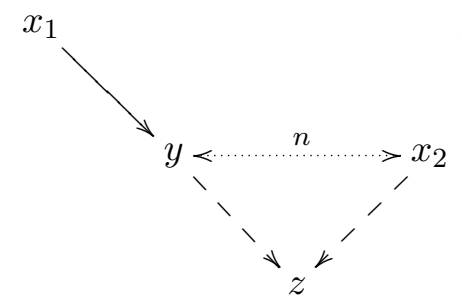


So ist in beiden Fällen die Church-Rosser Eigenschaft für $x_{1}$ und $x_{2}$ und deshalb auch für Wege der Länge $n+1$ erfüllt.

\section{Beispiel 1.1.16}

Folgendes ist ein noethersches Reduktionssystem auf $\mathcal{E}$, so dass es zu einem $x \in \mathcal{E}$ unendlich viele Church-Rosser-äquivalente Elemente gibt:

Sei

$\mathcal{E}:=\left\{x_{i} \mid i \in \mathbb{N}\right\} \cup\left\{z_{i} \mid i \in \mathbb{N}_{0}\right\}$ und $\mathcal{R}:=\left\{\left(x_{i}, z_{i}\right) \mid i \in \mathbb{N}\right\} \cup\left\{\left(x_{i}, z_{i-1} \mid i \in \mathbb{N}\right\}\right.$,

dann ist $x_{1}<\underset{\mathcal{R}}{>} x_{i}$ für jedes $i \in \mathbb{N}$, da:

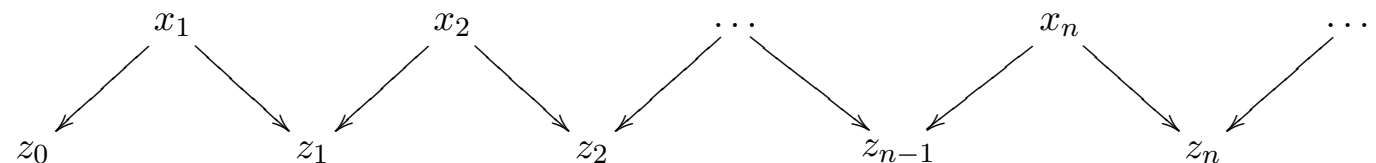

Wenn wir zusätzlich noch $z_{0}=z_{1}=\cdots=z_{n}=\ldots$ setzen, ist das Reduktionssystem sogar konvergent.

\subsubsection{Diamantenlemma}

Im Allgemeinen sind totale Konfluenz und die Church-Rosser Eigenschaft sehr schwer nachzuweisen. In diesem Abschnitt wollen wir zeigen, dass für noethersche Reduktionssysteme diese beiden Eigenschaften und lokale Konfluenz äquivalent sind.

Den folgenden Satz findet man in seiner Grundform in [New42]. Sein Name kommt daher, dass sich die Definitionen von lokaler Konfluenz, totaler Konfluenz und der Church-Rosser Eigenschaft leicht an einem Diamanten veranschaulichen lassen. Lokale Konfluenz bedeutet, dass je zwei Ecken, die von der Spitze über eine Kante erreichbar sind, über jeweils nur absteigende Kanten wieder verbunden werden können. Totale Konfluenz bedeutet, dass zwei beliebige Ecken, die über absteigenden Kanten von der Spitze erreichbar sind, über jeweils nur absteigende Kanten wieder verbunden werden können. Die Church-Rosser Eigenschaft bedeutet, dass zwei beliebige Ecken auf einem Diamanten über jeweils nur absteigende Kanten wieder verbunden werden 
können.

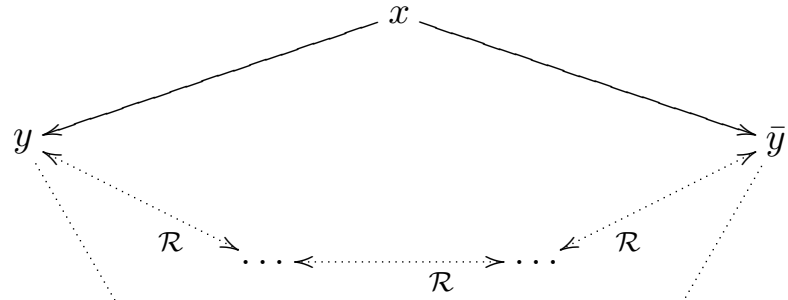

$z$

Satz 1.1.17 (Newmans Diamantenlemma)

Sei $\mathcal{R}$ noethersch, dann ist äquivalent:

1. Zu jedem $x \in \mathcal{E}$ gibt es genau eine Normalform.

2. Das Reduktionssystem $\mathcal{R}$ ist total konfluent.

3. Das Reduktionssystem $\mathcal{R}$ ist lokal konfluent.

4. Das Reduktionssystem $\mathcal{R}$ erfüllt die Church-Rosser Eigenschaft.

Beweis: [Satz 1.1.17] Nehmen wir also an, dass $\mathcal{R}$ noethersch ist, dann gibt es zu jedem Element aus $\mathcal{E}$ wenigstens eine Normalform.

- $1 \Rightarrow 2$ : Sei die Normalform eindeutig. Wir betrachten zwei Reduktionswege, die im selben $x$ beginnen und zu $y$ bzw. $\bar{y}$ reduzieren:

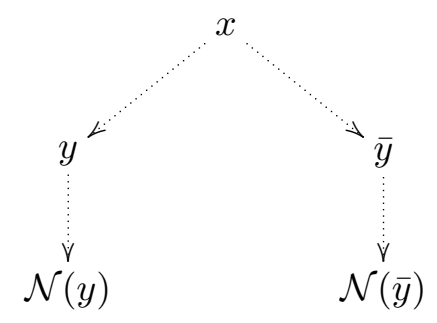

Da das Reduktionssystem noethersch ist, haben $y$ und $\bar{y}$ eine Normalform. Diese Normalformen sind auch Normalformen für $x$. Da die Normalform eindeutig ist, gilt $\mathcal{N}(y)=\mathcal{N}(\bar{y})$. Also ist das Reduktionssystem total konfluent. 
- $2 \Rightarrow 3$ : Zu zeigen: Aus total konfluent folgt lokal konfluent. Da Wege der Länge eins gerade Reduktionsregeln sind, ist jedes total konfluente auch ein lokal konfluentes Reduktionssystem.

- $3 \Rightarrow 1$ : Sei $\mathcal{R}$ lokal konfluent. Wir werden mit einem Widerspruchsbeweis zeigen, dass die Normalform eindeutig ist. Nehmen wir also an, dass es zwei unterschiedliche Normalformen $y_{n}$ und $\bar{y}_{m}$ zu einem $x$ gibt.

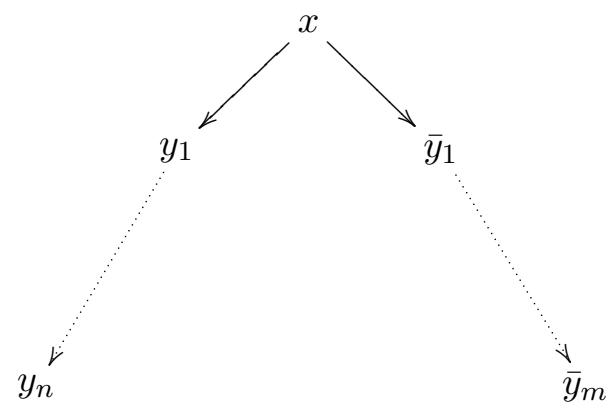

Da $\mathcal{R}$ lokal konfluent ist, lassen sich $y_{1}$ und $\bar{y}_{1}$ zu demselben Element $z_{1}$ reduzieren. Da $\mathcal{R}$ noethersch ist, gibt es eine Normalform $\mathcal{N}\left(z_{1}\right)$. Da sich $y_{n}$ und $\bar{y}_{m}$ unterscheiden, gilt $\mathcal{N}\left(z_{1}\right) \neq y_{n}$ oder $\mathcal{N}\left(z_{1}\right) \neq \bar{y}_{m}$. OBdA sei $\mathcal{N}\left(z_{1}\right) \neq y_{n}$. Dann hat auch $y_{1}$ zwei unterschiedliche Normalformen, nämlich $y_{n}$ und $\mathcal{N}\left(z_{1}\right)$.

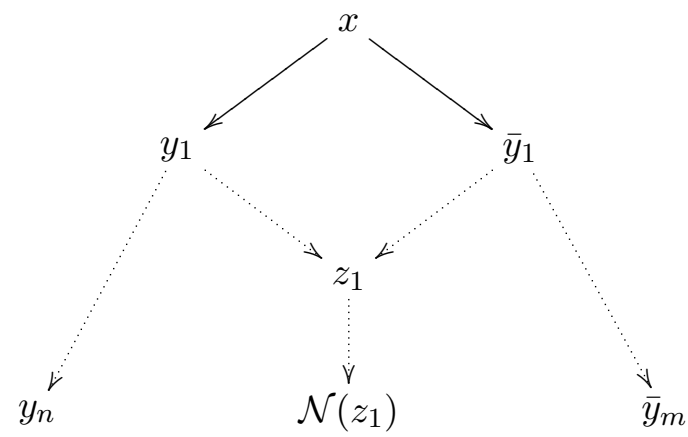

Nun nennen wir $y_{1}$ in $x_{1}$ um. Dann gilt: $x \longrightarrow x_{1}$, insbesondere $x \neq x_{1}$ und $x_{1}$ hat zwei Normalformen. Genauso wie für $x$ finden wir zu $x_{1}$ ein $x_{2}$, für welches gilt: $x_{1} \longrightarrow x_{2}$, insbesondere $x_{1} \neq x_{2}$ und $x_{2}$ hat zwei Normalformen. Durch Wiederholung erhalten wir einen unendlich langen Reduktionsweg:

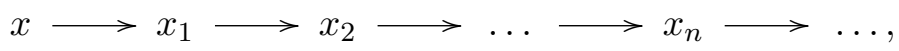

der ein Widerspruch zu noethersch ist.

- $3 \Leftrightarrow 4$ : Mit Lemma 1.1 .15 folgt die Behauptung.

\section{Bemerkung 1.1.18 (Konvergenz)}

Ein Reduktionssystem $\mathcal{R}$ heißt konvergent, wenn es noethersch ist und eine (und damit alle) der folgenden Bedingungen erfüllt: 
- Zu jedem $x \in \mathcal{E}$ gibt es genau eine Normalform.

- Das Reduktionssystem $\mathcal{R}$ ist total konfluent.

- Das Reduktionssystem $\mathcal{R}$ ist lokal konfluent.

- Das Reduktionssystem $\mathcal{R}$ erfüllt die Church-Rosser Eigenschaft.

\subsection{Reduktionssystem bezüglich einer Algebra}

In diesem Kapitel wollen wir eine Algebra $\boldsymbol{A}$ als Quotient einer freien Algebra $\mathcal{F}$ auffassen. So können wir jedes $a \in \boldsymbol{A}$ als Äquivalenzklasse in $\mathcal{F}$ betrachten. Wir werden $\mathcal{R}$ so wählen, dass $f_{1}, f_{2} \in \mathcal{F}$ genau dann in derselben Äquivalenzklasse liegen, wenn sie dieselbe Normalform haben. Die lokale Konfluenz dieses Reduktionssystems werden wir mittels minimaler Überschneidungen von Silben beweisen.

Die in dieser Arbeit betrachteten Spezialfälle von Reduktionssystemen nennt man auch Wortersetzungssysteme.

\subsubsection{Definitionen}

Sei $\mathbb{K}$ ein Körper. Sei $\mathcal{A}$ ein Alphabet.

Definition 1.2.1 (Terme $\mathcal{T}$ )

Ein (endliches) Produkt aus Elementen $a \in \mathcal{A}$ und einem Koeffizient $\lambda \in \mathbb{K}$ heißt Term. Die Menge der Terme bezeichnen wir mit $\mathcal{T}$, also:

$$
\mathcal{T}:=\left\{\lambda a_{1} \cdots a_{n} \mid a_{1}, \ldots, a_{n} \in \mathcal{A}, \lambda \in \mathbb{K}^{*}, n \in \mathbb{N}\right\} .
$$

Definition 1.2.2 (Monome $\mathcal{M}$ )

Terme ohne Koeffizient aus $\mathbb{K}$ heißen Monome oder Worte. Die Menge der Monome bezeichnen wir mit $\mathcal{M}$, also:

$$
\mathcal{M}:=\left\{a_{1} \cdots a_{n} \mid a_{1}, \ldots, a_{n} \in \mathcal{A}\right\} .
$$

Es ist auch das leere Monom zugelassen.

\section{Vereinbarung 1.2.3}

Im Folgenden werden wir nur noch Reduktionssysteme auf Mengen $\mathcal{E}$ betrachten, die $\mathcal{T}$ enthalten.

$$
\mathcal{M} \subset \mathcal{T} \subset \mathcal{E},
$$

wobei wir $\mathcal{E}$ später genauer festlegen werden.

Definition 1.2.4 (Wortersetzungssystem)

Ein Reduktionssystem $r$, in dem bei jeder Reduktionsregel auf der linken Seite ein Monom steht, heißt Wortersetzungssystem, also:

$$
r \subset \mathcal{M} \times \mathcal{E} .
$$




\section{Definition 1.2.5 (Algebra $A$ )}

Ein $\mathbb{K}$-Vektorraum $\boldsymbol{A}$ mit einer $\mathbb{K}$-bilinearen Verknüpfung

$$
\boldsymbol{A} \times \boldsymbol{A} \rightarrow \boldsymbol{A},
$$

für die zusätzlich gilt:

- Assoziativität: $a *(b * c)=(a * b) * c \quad \forall a, b, c \in \boldsymbol{A}$,

- Eins: $\exists 1 \in \boldsymbol{A}: 1 * a=a * 1=a \quad \forall a \in \boldsymbol{A}$,

heißt Algebra $\boldsymbol{A}$.

Definition 1.2.6 (freie Algebra $\mathcal{F}$ )

Sei $\mathcal{A}$ ein Alphabet und sei $\mathcal{M}$ die Menge von Monomen über $\mathcal{A}$. Wir bezeichnen als freie Algebra $\mathcal{F}$ den Ring:

$$
\mathcal{F}:=\left\{\begin{array}{l|l}
\sum_{m \in \mathcal{M}} \lambda_{m} m & \begin{array}{c}
\#\left\{\lambda_{m} \neq 0\right\}<\infty \\
\lambda_{m} \in \mathbb{K} \\
m \in \mathcal{M}
\end{array}
\end{array}\right\} .
$$

Für ein $f \in \mathcal{F}$ bezeichnen wir mit supp $\{f\}$ die Menge von Monomen, deren Koeffizient in $f$ ungleich Null ist.

Also sind die Monome über dem Alphabet $\mathcal{A}$ eine lineare Basis für $\mathcal{F}$. Manchmal schreiben wir für eine freie Algebra $\mathcal{F}_{n}$ um deutlich zu machen, dass es sich um die freie Algebra über einem Alphabet mit $n$ Buchstaben handelt.

Aus einem Wortersetzungssystem $r \subset \mathcal{M} \times \mathcal{F}$ lässt sich ein größeres Reduktionssystem auf der freien Algebra definieren.

Definition 1.2.7 (induziertes Reduktionssystem $\mathcal{R}$ )

Sei $r \subset \mathcal{M} \times \mathcal{F}$ ein Wortersetzungssystem, dann definieren wir:

$$
\mathcal{R}:=\left\{\begin{array}{l|c}
(\lambda p x s+q, \lambda p(y) s+q) & \begin{array}{c}
\lambda \in \mathbb{K} \\
(x, y) \in r \\
p, s \in \mathcal{M} \\
q \in \mathcal{F} \text { mit } p x s \notin \operatorname{supp}\{q\}
\end{array}
\end{array}\right\} .
$$

Wir sagen, $\mathcal{R}$ ist das durch $r$ auf $\mathcal{F}$ induzierte Reduktionssystem. Um deutlich zu machen, dass $y$ ein Polynom und nicht nur ein Monom wie $x$ ist, setzen wir Klammern.

In keinem Summanden, der in $q$ vorkommt, darf $p x s$ als Wort vorkommen, da sonst dieser Summand mit $\lambda p x s$ zusammengefasst werden könnte. Für Beweise ist es umständlich jeweils pxs $\notin \operatorname{supp}\{q\}$ zu formulieren (vergl. [Ber78b]). Im Folgenden übertragen wir daher $\mathcal{R}$ in ein größeres Reduktionssystem $\widetilde{\mathcal{R}}$, das auf einer größeren Menge definiert ist, in der mit + nicht zusammengefasst werden kann. Das Reduktionssystem $\widetilde{\mathcal{R}}$ soll so gewählt werden, dass sich die Menge der unreduzierbaren Elemente nicht verändert. Es sollen alle Regeln aus $\mathcal{R}$ enthalten sein, also müssen wir zusätzlich Regeln finden, die dem Zusammenfassen von Summanden in $\mathcal{F}$ entsprechen. Es verlängern sich die Reduktionswege, wodurch die Argumente deutlicher werden. 
Definition 1.2.8 ( $\operatorname{Rig} R$ )

Ein Rig ist eine Menge $\boldsymbol{R}$, die mit zwei Verknüpfungen Addition ${ }^{\prime}{ }^{\prime}$ und Multiplikation - versehen ist, so dass gilt:

- $\left(\boldsymbol{R},+^{\prime}\right)$ ist ein kommutativer Monoid mit neutralem Element 0:

$$
\begin{aligned}
\left(a++^{\prime} b\right)+^{\prime} c & =a+{ }^{\prime}\left(b+{ }^{\prime} c\right), \\
0+^{\prime} a & =a+{ }^{\prime} 0=a, \\
a++^{\prime} b & =b+{ }^{\prime} a .
\end{aligned}
$$

- $(\boldsymbol{R}, \cdot)$ ist ein Monoid mit neutralem Element 1:

$$
\begin{aligned}
(a \cdot b) \cdot c & =a \cdot(b \cdot c), \\
1 \cdot a & =a \cdot 1=a .
\end{aligned}
$$

- Distributiv:

$$
\begin{aligned}
& a \cdot\left(b+^{\prime} c\right)=(a \cdot b)++^{\prime}(a \cdot c), \\
& \left(a++^{\prime} b\right) \cdot c=(a \cdot c)+^{\prime}(b \cdot c) .
\end{aligned}
$$

- Null ist ein Annulator:

$$
0 \cdot a=a \cdot 0=0 .
$$

Wir wollen im Folgenden einen speziellen Rig betrachten. Dieser Rig soll die freie Algebra $\mathcal{F}$ enthalten. Dazu betrachten wir zunächst folgendes Beispiel.

\section{Beispiel 1.2.9}

Sei $(M, 1, \cdot)$ ein Monoid und sei $\left(H(M), 0,+^{\prime}\right)$ ein freier kommutativer durch $M$ erzeugter Monoid. Also ist $H(M)$ die Menge von formalen Summen

$$
m_{1}+{ }^{\prime} m_{2}+^{\prime} \cdots+{ }^{\prime} m_{k}
$$

von Elementen aus $M$.

Die Menge $\left(H(M), 1,0,+^{\prime}, \cdot\right)$ hat die Struktur eines Rigs, wobei die Multiplikation aus $M$ auf $H(M)$ erweitert wird:

$$
\left(m_{1}+{ }^{\prime} m_{2}+{ }^{\prime} \cdots+{ }^{\prime} m_{k}\right)\left(n_{1}+{ }^{\prime} n_{2}+{ }^{\prime} \cdots+{ }^{\prime} n_{l}\right):=\sum_{i=1}^{k}, \sum_{j=1}^{l}{ }^{\prime} m_{i} n_{j} .
$$

\section{Beispiel 1.2.10}

Sei $H$ wie in Beispiel 1.2.9 definiert. Die Menge von Termen $\mathcal{T}:=\left\{\lambda_{w} w \mid w \in \mathcal{M}\right\}$ ist über

$$
\lambda_{w} w \cdot \lambda_{\bar{w}} \bar{w}=\left(\lambda_{w} \lambda_{\bar{w}}\right) w \bar{w}
$$

ein Monoid, daher ist $H(\mathcal{T})$ ein Rig. 
Definition 1.2.11 $(\operatorname{Rig} \widetilde{\mathcal{F}})$

Sei $\mathcal{T}$ die Menge von Termen über einem Alphabet $\mathcal{A}$. Wir definieren den Rig über $\mathcal{A}$ durch:

$$
\widetilde{\mathcal{F}}:=H(\mathcal{T}),
$$

wobei $H$ wie in Beispiel 1.2 .9 definiert ist. Im Folgenden sprechen wir auch von dem Rig $\widetilde{\mathcal{F}}$, wenn eindeutig ist, welches $\mathcal{A}$ gemeint ist.

Wir können den $\operatorname{Rig} \widetilde{\mathcal{F}}$ auch auffassen als:

$$
\widetilde{\mathcal{F}}:=\left\{\begin{array}{l|l}
\sum_{w \in \mathcal{M}} \prime \sum_{i=1}^{k_{w}} \lambda^{\prime} \lambda_{w, i} w & \begin{array}{c}
\#\left\{k_{w} \neq 0\right\}<\infty \\
\lambda_{w, i} \in \mathbb{K}^{*} \\
w \in \mathcal{M}
\end{array}
\end{array}\right\} .
$$

Ein Element $\widetilde{f} \in \widetilde{\mathcal{F}}$ ist eine Menge von Familien ${ }^{2}$ über $\mathcal{M}$ mit Elementen in $\mathbb{K}^{*}$. Also ist $\tilde{f}$ ein Ausdruck von Summen mit Termen, die mit „, $+^{\prime \prime}$ anstelle von „,+ "verknüpft sind. Im Gegensatz zur Summe in der freien Algebra können die Koeffizienten vor gleichen Monomen nicht zusammengefasst werden. Es ist also zu beachten, dass diese Summe nicht distributiv mit der Multiplikation des Körpers ist, also:

$$
\lambda w+^{\prime} \mu w \neq(\lambda+\mu) w .
$$

Zwei Ausdrücke sind dann gleich, wenn sie die gleichen Summanden in höchstens unterschiedlicher Reihenfolge besitzen.

\section{Vereinbarung 1.2.12}

Wir betrachten $\mathcal{F} \subset \widetilde{\mathcal{F}}$ in folgender Weise:

$$
\text { Sei } f=\sum_{w \in \mathcal{M}} \alpha_{w} w \in \mathcal{F} \text {, dann ist } f=\sum_{w \in \mathcal{M}}{ }^{\prime} \sum_{i=1}^{k_{w}}{ }^{\prime} \alpha_{w} w \in \widetilde{\mathcal{F}},
$$

wobei $k_{w}=\left\{\begin{array}{cc}0 & \text { für } \alpha_{w}=0 \\ 1 & \text { sonst }\end{array}\right.$. Bei dieser Einbettung ist zu beachten, dass sie mit den Algebra-Verknüpfungen nicht verträglich ist.

Wir wollen nun ein größeres Reduktionssystem definieren, das $\mathcal{R}$ enthält.

\section{Definition 1.2.13 (induziertes Reduktionssystem $\widetilde{\mathcal{R}}$ )}

Sei $r \mathcal{M} \times \mathcal{F}$ ein Wortersetzungssystem und sei:

$$
\begin{aligned}
& \operatorname{Rel}:=\left\{\begin{array}{l|l}
\left(\lambda p x s+{ }^{\prime} q, \lambda p(y) s+^{\prime} q\right) & \begin{array}{c}
\lambda \in \mathbb{K}^{*} \\
p, s \in \mathcal{M} \\
q \in \widetilde{\mathcal{F}} \\
(x, y) \in r
\end{array}
\end{array}\right\}, \\
& \widetilde{\operatorname{Rel}}:=\left\{\begin{array}{l|l}
\left(\lambda w+^{\prime} \mu w+^{\prime} q,(\lambda+\mu) w+^{\prime} q\right) & \begin{array}{c}
\lambda, \mu \in \mathbb{K}^{*} \\
w \in \mathcal{M} \\
q \in \widetilde{\mathcal{F}}
\end{array}
\end{array}\right\} .
\end{aligned}
$$

Das durch

$$
\widetilde{\mathcal{R}}:=\operatorname{Rel} \cup \widetilde{\operatorname{Rel}}
$$

definierte Reduktionssystem heißt das durch $r$ auf $\widetilde{\mathcal{F}}$ induzierte.

\footnotetext{
${ }^{2}$ In einer Familie kann ein Element mehrmals vorkommen.
} 


\subsubsection{Konfluenz}

Sei $\mathcal{R}$ bzw. $\widetilde{\mathcal{R}}$ das durch $r$ auf $\mathcal{F}$ bzw. $\widetilde{\mathcal{F}}$ induzierte Reduktionssystem. In diesem Abschnitt zeigen wir, dass die Mengen von unreduzierbaren Elementen aus $\mathcal{R}$ und aus $\widetilde{\mathcal{R}}$ übereinstimmen und dass sich Konfluenz von $\widetilde{\mathcal{R}}$ nach $\mathcal{R}$ überträgt, falls $\widetilde{\mathcal{R}}$ noethersch ist.

$\operatorname{Satz} 1.2 .14(\mathcal{N}=\tilde{\mathcal{N}})$

Sei $\mathcal{N}$ die Menge von unreduzierbaren Elementen in $\mathcal{F}$ bezüglich $\mathcal{R}$ und sei $\widetilde{\mathcal{N}}$ die Menge der unreduzierbaren Elemente in $\widetilde{\mathcal{F}}$ bezüglich $\widetilde{\mathcal{R}}$. Es gilt:

$$
\tilde{\mathcal{N}} \subseteq \mathcal{F}
$$

und

$$
\mathcal{N}=\tilde{\mathcal{N}}
$$

Beweis: [Satz 1.2.14] Sei $f \in \widetilde{\mathcal{N}}$. In $f$ können keine zwei Terme mit gleichen Mono-

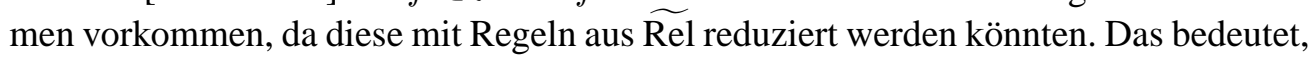
dass $f$ in $\mathcal{F}$ liegt.

Für den zweiten Teil des Satzes bemerken wir zunächst, dass $z \in \mathcal{N}$ genau dann gilt, wenn $z$ ein Polynom in $\mathcal{F}$ ist, in dem kein Summand eine Silbe enthält, die sich mit einer Regel aus $r$ reduzieren lässt. Mit 1.2.12 können wir $z$ als ein Element aus $\widetilde{\mathcal{F}}$ auffassen, für welches keine zwei Terme mit gleichen Monomen vorkommen. Es kann also keine Regel aus $\widetilde{\mathcal{R}}$ angewendet werden. Also ist $z$ auch in $\widetilde{\mathcal{F}}$ ein unreduzierbares Element.

Für die umgekehrte Richtung sei $z \in \widetilde{\mathcal{N}}$. Das ist genau dann der Fall, wenn weder

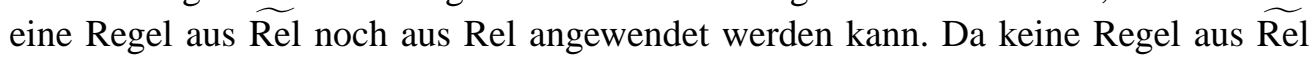
angewendet werden kann, können in $z$ keine zwei Terme mit gleichen Monomen vorkommen, wir können $z$ also auch als Element in $\mathcal{F}$ auffassen. Da nach Voraussetzung auch keine Regel aus Rel angewendet werden kann, können wir auch keine Regel aus $\mathcal{R}$ anwenden, also gilt $z \in \mathcal{N}$.

Ein Reduktionsweg in $\widetilde{\mathcal{R}}$, der in $\mathcal{F}$ beginnt und endet, impliziert jedoch keinen entsprechenden Reduktionsweg $\mathcal{R}$. Dazu betrachten wir folgendes Beispiel.

\section{Beispiel 1.2.15}

Sei $r$ ein Wortersetzungssystem, das nur aus folgenden zwei Regeln besteht:

$$
x \longrightarrow{ }_{r} z+y \text { und } y \longrightarrow{ }_{r} \text {. }
$$

Dann gibt es einen Reduktionsweg in $\widetilde{\mathcal{R}}$ :

$$
x+^{\prime}(-y) \longrightarrow \widetilde{\mathcal{R}}\left(z+^{\prime} y\right)+^{\prime}(-y) \longrightarrow \overrightarrow{\mathcal{R}}\left(z+^{\prime} \bar{z}\right)+^{\prime}(-y),
$$

der in $\mathcal{F}$ beginnt und endet; aber es gibt keinen entsprechenden Reduktionsweg in $\mathcal{R}$, 
da es für $x-y$ in $\mathcal{R}$ nur folgende Reduktionswege gibt:

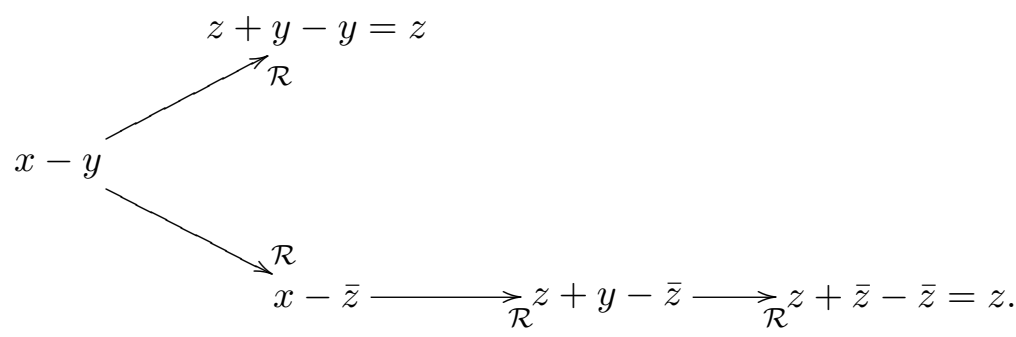

Die umgekehrte Richtung gilt jedoch. Dazu betrachten wir folgendes Lemma.

Lemma 1.2.16 $\left(\cdots \cdots \cdots,>_{\mathcal{R}} \subset \quad \cdots \cdots\right.$

Zu jedem Reduktionsweg in $\mathcal{R}$ gibt es einen Reduktionsweg in $\widetilde{\mathcal{R}}$.

Beweis: [Lemma 1.2.16] Direkt aus der Definition 1.2.13 folgt, dass jede Regel aus $\mathcal{R}$ sich durch eine Regel aus Rel und mehrere Regeln aus Rel schreiben lässt. Also lässt sich auch jeder Weg in $\mathcal{R}$ in einen (eventuell längeren) Weg in $\widetilde{\mathcal{R}}$ übersetzen.

Satz 1.2.17 (Konvergenz)

Sei $\widetilde{\mathcal{R}}$ konvergent, dann ist auch $\mathcal{R}$ konvergent .

Beweis: [Satz 1.2.17

Sei $\widetilde{\mathcal{R}}$ noethersch. Wegen Lemma 1.2.16 lässt sich jede Reduktionsregel in $\mathcal{R}$ in eine

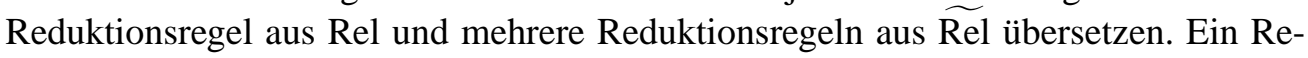
duktionsweg $\cdots \cdots \cdots \mathcal{R}_{\mathcal{R}}$ in $\mathcal{F}$ kann demnach in einen längeren Reduktionsweg $\cdots \cdots \cdots \widetilde{\mathcal{R}}^{\rightarrow}$ in $\widetilde{\mathcal{F}}$ überführt werden. Wenn dieser längere Reduktionsweg endlich ist, dann ist es auch der in $\mathcal{F}$. Also ist $\mathcal{R}$ noethersch. Daraus folgt: Jedes $f \in \mathcal{F}$ hat wenigstens eine Normalform bezüglich $\mathcal{R}$.

Für die Konfluenz betrachten wir folgenden Widerspruchsbeweis:

Sei nun $\mathcal{R}$ nicht konfluent, dann gibt es ein $f \in \mathcal{F}$ mit zwei verschiedenen Normalformen $z_{1}$ und $z_{2}$ bezüglich $\mathcal{R}$. Es gibt also zwei Reduktionswege: $f \quad \ldots \ldots \ldots \overrightarrow{\mathcal{R}}_{\mathcal{R}} z_{1}$ und $f \quad \ldots . . . . .{ }_{\mathcal{R}} z_{2} . \mathrm{Zu}$ diesen Reduktionswegen in $\mathcal{R}$ können wir mit 1.2 .16 Reduktionswege in $\widetilde{\mathcal{R}}$ finden. Nun sind $z_{1}$ und $z_{1}$ nach 1.2 .14 unreduzierbar bezüglich $\widetilde{\mathcal{R}}$. Dies ist ein Widerspruch zur Konfluenz von $\widetilde{\mathcal{R}}$.

Bemerkung 1.2.18 (Induzierter Reduktionsweg)

Sei $g_{1} \quad \cdots \ldots \ldots \ldots$ …

$$
a g_{1} b+^{\prime} q \quad \cdots \cdots \cdots \underset{\widetilde{\mathcal{R}}}{\underset{2}{*}} a g_{2} b+^{\prime} q
$$

ein Reduktionsweg, wobei $a, b, q$ beliebige Elemente aus dem Rig $\widetilde{\mathcal{F}}$ sein können.

\subsection{3 Überschneidungen}

Im letzten Abschnitt hatten wir gesehen, dass es reicht die Konvergenz von $\widetilde{\mathcal{R}}$ zu zeigen um die Konvergenz von $\mathcal{R}$ zu beweisen. Jedoch ist es viel Arbeit alle möglichen 
Reduktionswege von jedem Element aus $\widetilde{\mathcal{F}}$ zu überprüfen. Daher wollen wir nun zeigen, dass es für den Beweis der Konfluenz ausreicht sich auf eine Teilmenge zu beschränken. Wie auch in den vorhergehenden Abschnitten seien $\mathcal{R}$ und $\widetilde{\mathcal{R}}$ durch ein Wortersetzungssystem $r$ induziert.

Definition 1.2.19 (Überschneidung $(w, V, \bar{V})$ )

Eine Überschneidung $(w, V, \bar{V})$ ist ein Monom $w \in \widetilde{\mathcal{F}}$, zusammen mit zwei unterschiedlichen Reduzierungsregeln $V=(x, y), \bar{V}=(\bar{x}, \bar{y}) \in r$, so dass es $p, \bar{p}, s, \bar{s} \in$ $\mathcal{M}$ und $\lambda, \bar{\lambda} \in \mathbb{K}^{*}$ und $q, \bar{q} \in \widetilde{\mathcal{F}}$ gibt mit:

$$
w=\lambda p x s+{ }^{\prime} q=\bar{\lambda} \bar{p} \bar{x} \bar{s}+^{\prime} \bar{q} .
$$

\section{Bemerkung 1.2.20 (leeres Monom)}

Die Präfixe $p, \bar{p}$ und die Suffixe $s, \bar{s}$ können auch das leere Wort sein.

Manche Überschneidungen lassen sich durch kürzere ersetzen. Betrachtet man zum Beispiel die Überschneidung $p x=\bar{p} \bar{x}$, dann beginnen die Monome $p, \bar{p}$ gleich, sie lassen sich also zerlegen in $p=q \cdot p_{1}$ und $\bar{p}=q \cdot \bar{p}_{1}$, wobei $p_{1}$ oder $\bar{p}_{1}$ leer ist. Ähnliches gilt für Suffixe. Daher ist folgende Definition nahe liegend.

\section{Definition 1.2.21 (minimale Überschneidung, kritisches Paar)}

Eine minimale Überschneidung $(w, V, \bar{V})$ ist ein Monom $w \in \mathcal{M}$, zusammen mit zwei unterschiedlichen Reduzierungsregeln $V=(x, y), \bar{V}=(\bar{x}, \bar{y}) \in r$, so dass es $p, s \in \mathcal{M}$ gibt mit:

$$
w=p x=\bar{x} s \quad \text { oder } \quad w=p x s=\bar{x} .
$$

Eine minimale Überschneidung besteht also maximal aus einem Präfix und einem Suffix. Manchmal sagen wir zu einer minimalen Überschneidung auch kritisches Paar.

Definition 1.2.22 (zusammenführbar, behebbar)

Eine minimale Überschneidung $\left(w, V_{1}, \bar{V}_{1}\right)$ heißt zusammenführbar oder behebbar bezüglich $\mathcal{R}$ bzw. $\widetilde{\mathcal{R}}$, wenn es ein $z \in \mathcal{F}$ bzw. $\in \widetilde{\mathcal{F}}$ zusammen mit zwei endlichen Reduktionswegen $\left(V_{i}\right)_{i=1 \ldots n}$ und $\left(\bar{V}_{i}\right)_{i=1 \ldots \bar{n}}$ in $\mathcal{R}$ bzw. $\widetilde{\mathcal{R}}$ gibt, die im selben Element $z$ enden und es somit Wege gibt, die die beiden Regeln wieder zusammenführen.

\section{Definition 1.2.23 (vollständig)}

Ein Ersetzungssystem $r$ heißt vollständig bezüglich $\mathcal{R}$ bzw. $\widetilde{\mathcal{R}}$, falls jede minimale Überschneidung mit Reduktionsregeln aus $\mathcal{R}$ bzw. $\widetilde{\mathcal{R}}$ behebbar ist.

Satz 1.2.24 ( $r$ vollständig $\Rightarrow \widetilde{\mathcal{R}}$ lokal konfluent)

Sei $\widetilde{\mathcal{R}}$ noethersch. Falls $r$ vollständig bezüglich $\widetilde{\mathcal{R}}$ ist, dann gilt:

$$
\widetilde{\mathcal{R}} \text { ist lokal konfluent. }
$$

Beweis: [Satz 1.2.24 Seien alle minimalen Überschneidungen aus $r$ behebbar. Es muss gezeigt werden, dass es zu jedem $g_{1} \in \widetilde{\mathcal{F}}$, zu dem es in $\widetilde{\mathcal{R}}$ zwei verschiedene

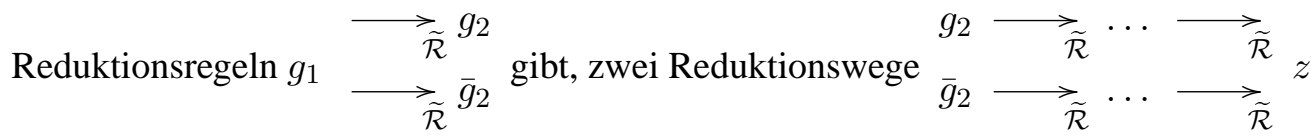
gibt, die im gleichen $z$ enden. 
Die Reihenfolge der Summanden spielt keine Rolle. Deshalb schreiben wir die zu betrachtenden Summanden immer an den Anfang. Sei $(x, y)$ eine Reduktionsregel aus $r$. Für die Reduktionsregel in Rel schreiben wir dann:

$$
p x s+^{\prime} q \longrightarrow \underset{\widetilde{\mathcal{R}}}{\longrightarrow} p(y) s+^{\prime} q .
$$

Sei $(\bar{x}, \bar{y})$ eine weitere Reduktionsregel aus $r$.

Wir müssen folgende Arten von Überschneidungen betrachten:

- Beginnend mit Reduktionsregeln jeweils aus Rel:

Eine Reduktionsregel aus Rel überführt ein ${ }^{\prime}{ }^{\prime}$ in die übliche Addition. Die Reihenfolge, in der Reduktionsregeln aus $\widetilde{R e l}$ angewendet werden, spielt also keine Rolle.

- Beginnend mit einer Reduktionsregel aus Rel und einer aus Rel:

Wir weisen für die unterschiedlichen Fälle nach, dass sie zum gleichen Element reduziert werden können.

Fall „Getrennt“: Sei $g_{1}=\lambda p x s+{ }^{\prime} \mu \bar{x}+{ }^{\prime} \bar{\mu} \bar{x}+{ }^{\prime} q$.

Hier betrachten wir:

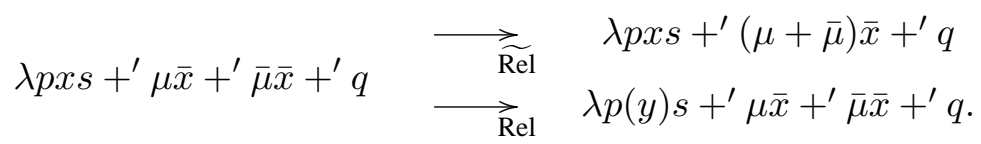

Diese Elemente lassen sich mit Regeln aus Rel bzw. $\widetilde{\text { Rel }}$ weiter reduzieren zu jeweils $\lambda p(y) s+^{\prime}(\mu+\bar{\mu}) \bar{x}+^{\prime} q$.

Fall „Inverses“: Sei $g_{1}=\lambda p x s+^{\prime}(-\lambda p x s)+^{\prime} q$.

Hier betrachten wir:

$$
\lambda p x s+^{\prime}(-\lambda p x s)+^{\prime} q \underset{\text { Rel }}{\stackrel{\overrightarrow{\mathrm{Rel}}}{\longrightarrow}} \lambda p(y) s+^{\prime}(-\lambda p x s)+^{\prime} q .
$$

Der untere Teil lässt sich mit Regeln aus Rel weiter reduzieren zu $\lambda p(y) s+^{\prime}$ $(-\lambda p(y) s)+{ }^{\prime} q$ und dann mit Regeln aus $\widetilde{\operatorname{Rel}}$ zu $q$ reduzieren.

Fall „Überlagert“: Sei $g_{1}=\lambda p x s+{ }^{\prime} \mu p x s+^{\prime} q$.

Hier betrachten wir:

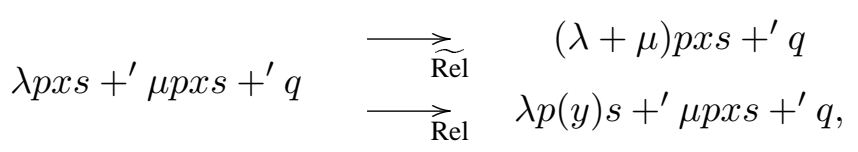

da gilt:

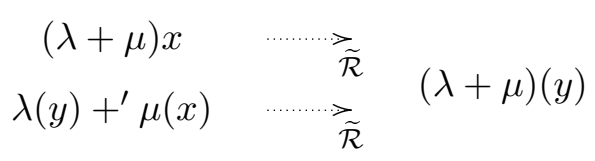

und wegen Bemerkung 1.2.18 lässt sich diese Überschneidung zum selben Element zusammenführen. 
- Beginnend mit zwei Reduktionsregeln aus Rel:

Fall „Getrennt“: Sei $g_{1}=\lambda p x s+{ }^{\prime} \mu \bar{p} \bar{x} \bar{s}+{ }^{\prime} q$.

Hier betrachten wir:

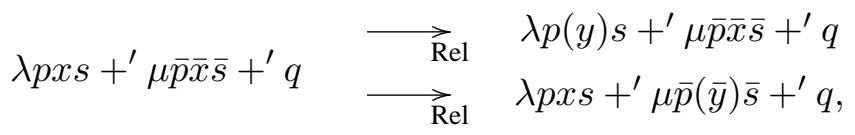

da gilt:

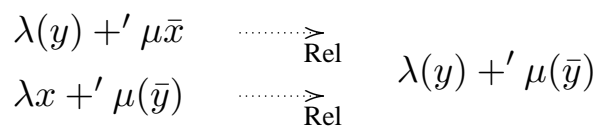

und wegen Bemerkung 1.2.18 lässt sich diese Überschneidung zum selben Element zusammenführen.

Fall: „Überschneidung“: Sei $g_{1}=\lambda p x s \bar{x} \bar{s}+{ }^{\prime} q$.

Hier betrachten wir:

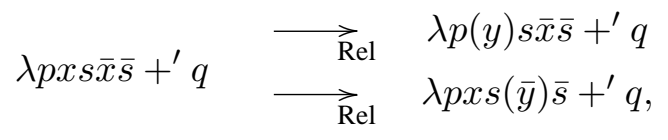

da gilt:

$$
\begin{array}{ll}
(y) s \bar{x} & \overrightarrow{\mathrm{Rel}} \\
x s(\bar{y}) & \mathrm{R} \mathrm{el}
\end{array}
$$

und wegen Bemerkung 1.2.18 lässt sich diese Überschneidung zum selben Element zusammenführen.

Dies waren die Fälle, die unabhängig davon sind, ob die minimalen Überschneidungen in $r$ behebbar sind. Sei nun jede minimale Überschneidung aus $r$ mit Regeln aus $\mathcal{R}$ behebbar. Mit Satz 1.2.16 gibt es dann auch einen Reduktionsweg in $\widetilde{\mathcal{R}}$, der die minimale Überschneidung behebt. Sei $(x, y),(\bar{x}, \bar{y}) \in r$. Wir betrachten noch folgende Fälle:

- minimale Teilüberschneidung: Sei $f=p x=\bar{x} s$.

Hier betrachten wir:

$$
\begin{array}{ccc}
\lambda p x b+^{\prime} q & \longrightarrow \text { Rel } & \lambda p(y) b+^{\prime} q \\
\longrightarrow & \text { Rel } & \lambda a(\bar{y}) s b+^{\prime} q,
\end{array}
$$

da nach Voraussetzung

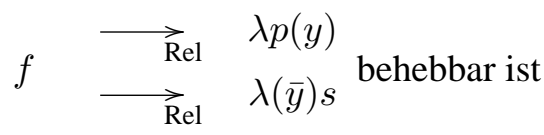

und wegen Bemerkung 1.2.18 lässt sich diese Überschneidung zum selben Element zusammenführen. 
- minimale Totalüberschneidung: Sei $f=p x s=\bar{x}$.

Hier betrachten wir:

$$
\begin{array}{ccc}
\lambda a p x s b+{ }^{\prime} q & \overrightarrow{\operatorname{Rel}} & \lambda a p(y) s b+^{\prime} q \\
& \overrightarrow{\operatorname{Re}} & \lambda a(\bar{y}) b+^{\prime} q,
\end{array}
$$

da nach Voraussetzung:

$$
f \quad \begin{array}{ll}
\longrightarrow \\
\overrightarrow{\operatorname{Re} e l} & \lambda p(y) s \\
\longrightarrow & \lambda(\bar{y})
\end{array} \text { behebbar ist }
$$

und wegen Bemerkung 1.2.18 lässt sich diese Überschneidung zum selben Element zusammenführen.

Wir haben nun nachgewiesen, dass sich das Element für alle Überschneidungen, egal mit welcher Regel begonnen wird, auf dasselbe Element reduzieren lässt.

\subsubsection{Ordnungen}

Im vorhergehenden Kapitel haben wir gesehen, wie wir lokale Konfluenz nachweisen können. Als nächstes wollen wir eine Methode entwickeln, wie wir noethersch nachweisen können.

Dazu werden wir für den Rig $\widetilde{\mathcal{F}}$ eine Ordnung definieren. Die Kunst besteht darin eine geeignete Ordnung auszuwählen, damit die Konvergenz von $\widetilde{\mathcal{R}}$ leicht zu beweisen ist.

\section{Definitionen}

\section{Definition 1.2.25 (Striktordnung)}

Eine strikte Teilordnung, oder kurz Striktordnung, ist eine Relation $<$ auf einer Menge $M$, die irreflexiv ( $a \nless a$ für alle $a \in M$ ) und transitiv ( $a<b$ und $b<c$, dann $a<c$ für alle $a, b, c \in M)$ ist.

\section{Definition 1.2.26 (Quasiordnung)}

Eine Quasiordnung ist eine Relation $\lesssim$ auf einer Menge $M$, die reflexiv ( $a \lesssim a$ für alle $a \in M$ ) und transitiv ist.

Es ist stets möglich, aus einer Quasiordnung eine Äquivalenzrelation zu extrahieren, mittels:

$$
a \approx b: \Leftrightarrow a \lesssim b \text { und } b \lesssim a .
$$

Der verbleibende, strikte Anteil ist dann definiert durch:

$$
a<b: \Leftrightarrow a \lesssim b \text { und nicht } b \lesssim a .
$$

Äquivalenzrelation und strikter Anteil geben gemeinsam die Quasiordnung vollständig wieder. Offensichtlich ist eine Quasiordnung genau dann eine Teilordnung, wenn ihre Äquivalenzrelation die Gleichheit ist. 
Definition 1.2.27 (Wohlordnung)

Eine Striktordnung $<$ auf $M$ heißt (strikte) Wohlordnung, falls folgende Bedingungen erfüllt sind:

- Strikte Totalordnung: Für jedes Paar $a, b \in M$ ist entweder $a=b$ oder $a<b$ oder $b<a$.

- Noethersch: Es gibt keine unendliche, strikt absteigende Folge in $M$. Äquivalent dazu: Jede absteigende Folge wird stationär, d.h.

$$
\left(a_{j}\right)_{j \in \mathbb{N}} \subset M \text { mit } a_{j} \leq a_{j+1} \forall j \in \mathbb{N} \Rightarrow \exists N \in N \forall j \geq N: a_{j}=a_{N} .
$$

Eine Quasiordnung heißt noethersch, wenn ihr strikter Anteil noethersch ist.

Definition 1.2.28 (Multiplikativität)

Eine Quasiordnung $\lesssim$ auf einer Halbgruppe $M$ (bspw. die Menge der Monome über einem Alphabet) heißt multiplikativ, wenn für alle $p, a, b, s \in M$ gilt:

$$
\begin{array}{ll}
a<b \Rightarrow p a<p b & \text { (Präfix-Invarianz) } \\
a & \approx b \Rightarrow p a \approx p b \\
\text { und } \quad a<b \Rightarrow a s<b s & \\
& a \approx b \Rightarrow a s \approx b s \quad \text { (Suffix-Invarianz). }
\end{array}
$$

\section{Definition 1.2.29 (kombinierte Ordnung)}

Seien $\lesssim_{1}$ und $\lesssim_{2}$ zwei Quasiordnungen auf $M$. Dann definieren wir die kombinierte Ordnung $\lesssim 1,2$ durch:

$$
a \lesssim 1,2 b: \Leftrightarrow a<_{1} b \text { oder }\left(a \approx_{1} b \text { und } a \aleph_{2} b\right)
$$

In der kombinierten Ordnung $\lesssim 1,2$ wird zuerst nach der Ordnung $\lesssim 1$ sortiert. Erst wenn zwei Elemente bezüglich $\lesssim 1$ nicht angeordnet sind, wird $\lesssim 2$ zu Rate gezogen (s.a. [Ave95], Kapitel 1.3). Zwei Elemente sind bezüglich ${ }_{1,2}$ äquivalent, wenn sie sowohl in $\approx_{1}$ als auch in $\approx_{2}$ äquivalent sind.

\section{Satz 1.2.30 (kombinierte Ordnung)}

Die kombinierte Ordnung $\lesssim_{1,2}$ ist eine Quasiordnung.

Beweis: [Satz 1.2.30] Reflexivität: Folgt aus der Reflexivität von $\lesssim_{1}$ und $\varsigma_{2}$. Transitivität: Sei $a \lesssim{ }_{1,2} b \lesssim{ }_{1,2} c$ mit $a, b, c \in M$. Dann gibt es vier Fälle:

- $a<_{1} b<_{1} c: \quad a<_{1,2} c$ folgt direkt.

- $a \approx_{1} b<_{1} c:$ Es gilt $a \lesssim_{1} c$, aber nicht $a \approx_{1} c$, also $a<_{1} c$.

- $a<_{1} b \approx_{1} c$ : Wie Fall (2).

- $a \approx_{1} b \approx_{1} c:$ Es gilt $a \approx_{1} c$ und $a \lesssim_{2} c$. 
Sind $\lesssim_{1}$ und $\lesssim_{2}$ noethersch, so ist es auch $\lesssim_{1,2}$ :

Sei $\left(a_{j}\right) \subset \mathcal{A}$ eine in $\lesssim 1,2$ strikt absteigende Folge. Es kann nur eine endliche Unterfolge geben, die auch in $\lesssim_{1}$ strikt abfällt. Alle darauffolgenden Folgenglieder müssen in $\lesssim 2$ eine absteigende Folge bilden. Diese muss stationär werden, da $\lesssim 2$ noethersch ist.

\section{Satz 1.2.31 (multiplikativ)}

Sind $\lesssim_{1}$ und $\lesssim_{2}$ multiplikativ, so ist es auch $\lesssim_{1,2}$.

Beweis: [Satz 1.2.31]

Sei $p \in M$. Für die Präfix-Invarianz müssen wir die folgenden Fälle betrachten:

- $a<_{1,2} b$

Falls $a<_{1} b$, dann folgt $p a<_{1} p b$. Also gilt $p a<_{1,2} p b$.

Falls $a \approx_{1} b$ und $a<_{2} b$, dann ist $p a \approx_{1} p b$ und $p a<_{2} p b$, folglich gilt $p a<_{1,2} p b$.

- $a \approx_{1,2} b$, dann ist $a \approx_{1} b$ und $a \approx_{2} b$.

Es folgt, dass $p a \approx_{1} p b$ und $p a \approx_{2} p b$. Also gilt $p a \approx_{1,2} p b$.

Die Suffix-Invarianz beweist man analog.

Sei nun $(\mathcal{A},<)$ eine partiell geordnete Menge.

Definition 1.2.32 (protolexikografische Ordnung)

Die protolexikografische Ordnung auf den Monomen über $\mathcal{A}$ ist definiert durch:

$$
\begin{aligned}
& a_{1} \ldots a_{n}<_{\text {plex }} a_{1}^{\prime} \ldots a_{m}^{\prime} \\
& : \Leftrightarrow n=m \text { und } \begin{cases} & a_{1}<a_{1}^{\prime} \\
\text { oder } & a_{1}=a_{1}^{\prime}, a_{2}<a_{2}^{\prime} \\
\text { oder } & \ldots \\
\text { oder } & a_{1}=a_{1}^{\prime}, \ldots, a_{n-1}=a_{n-1}^{\prime}, a_{n}<a_{n}^{\prime}\end{cases}
\end{aligned}
$$

für beliebige $a_{j}, a_{j}^{\prime} \in \mathcal{A}, j=1 \ldots n$.

Diese Ordnung ist nur eine strikte Teilordnung, da Worte verschiedener Länge nicht verglichen werden können. Sie ist multiplikativ nach Definition. Allerdings ist $<_{\text {plex }}$ eine Totalordnung, wenn man sich auf Monome fester Länge beschränkt, und das Alphabet $\mathcal{A}$ total geordnet ist.

Ist $\mathcal{A}$ noethersch (z.B. weil $\mathcal{A}$ endlich ist), so ist auch < plex noethersch: Da wir nur Wörter gleicher und endlicher Länge vergleichen, muss jede absteigende Folge letztlich auf eine absteigende Folge von Buchstaben aus $\mathcal{A}$ an einer stationären Stelle in den Wörtern hinauslaufen.

\section{Definition 1.2.33 (lexikografische Ordnung)}

Die lexikografische Ordnung erhält man aus der protolexikografischen, indem man $\mathcal{A}_{e}=\mathcal{A} \cup\{e\}$ setzt, mit einem neuen kleinsten Element $e<a$ für alle $a \in \mathcal{A}$. Die lexikografische Ordnung auf $\mathcal{A}$ ist dann

$$
a<_{\operatorname{lex}} a^{\prime}: \Leftrightarrow a e^{\left|a^{\prime}\right|}<_{\text {plex }} a^{\prime} e^{|a|},
$$

wobei $<_{\text {plex }}$ die protolexikografische Ordnung auf $\mathcal{A}_{e}$ ist. D.h., es werden lediglich genügend viele $e$ an die Enden der Monome angehängt, um ihre Längen anzugleichen. 
Falls $\mathcal{A}$ total geordnet ist, ist auch die lexikografische Ordnung eine totale Ordnung, aber nicht noethersch: Ein Gegenbeispiel ist die Folge $b>a b>a a b>a a a b>\ldots$ mit $a<b$. Sie ist auch nicht multiplikativ: Mit $a<b$ ist zwar $a<a a$, aber $a b>a a b$.

\section{Definition 1.2.34 (Knuth-Bendix-Ordnung)}

Die Knuth-Bendix-Ordnung auf den Monomen über $\mathcal{A}$ zu einer Gewichtsfunktion $g: \mathcal{A} \rightarrow \mathbb{N}_{+}$ist definiert durch:

$$
a \lesssim a^{\prime}: \Leftrightarrow G(a) \leq G\left(a^{\prime}\right)
$$

wobei $G\left(a_{1} \ldots a_{n}\right):=\sum_{j=1}^{n} g\left(a_{j}\right)$.

Die Knuth-Bendix-Ordnung ist eine Quasiordnung (mit reell-positiven Koeffizienten ist sie genau dann eine strikte Totalordnung, wenn die Menge der Gewichte $\left\{G\left(a_{j}\right)\right.$ : $\left.a_{j} \in \mathcal{A}\right\}$ linear unabhängig über $\mathbb{Q}$ ist). Sie ist offensichtlich multiplikativ, und auch noethersch, da die natürliche Ordnung auf $\mathbb{N}_{+}$noethersch ist.

\section{Definition 1.2.35 (Längenordnung)}

Die Längenordnung $\lesssim$ Länge auf den Monomen über $\mathcal{A}$ ist gegeben durch die KnuthBendix-Ordnung zur Gewichtsfunktion $G(a)=1 \forall a \in \mathcal{A}$.

Die Längenordnung zählt nur die Zahl der Buchstaben in einem Monom. Wie die allgemeine Knuth-Bendix-Ordnung ist auch die Längenordnung im nicht-trivialen Fall eine strikte Teilordnung, multiplikativ und noethersch. In der Längenordnung sind zwei Monome genau dann äquivalent, wenn sie die gleiche Länge besitzen.

\section{Definition 1.2.36 (kanonische Ordnung)}

Die kanonische Ordnung oder entgegengesetzt lexikographische Ordnung $\lesssim$ kan auf den Monomen über einer total geordneten Menge $\mathcal{A}$ ist $\lesssim$ Länge, plex.

Die kanonische Ordnung ist eine Teilordnung: Zwei Monome sind in ihr äquivalent, wenn sie gleiche Länge haben und aus den gleichen Buchstaben bestehen. Also ist $\approx_{\text {kan }}$ die Gleichheit. Sie ist sogar eine Totalordnung (wenn $\mathcal{A}$ total geordnet ist), da $<_{\text {plex }}$ eine Totalordnung auf den Mengen gleich langer Monome ist.

Sie ist zudem multiplikativ und für noethersches $\mathcal{A}$ auch noethersch, da sie eine Kombination von kürzbar-multiplikativen und noetherschen Ordnungen ist. Sie lässt sich auch analog zur lexikographischen Ordnung schreiben als

$$
a<_{\text {kan }} a^{\prime}: \Leftrightarrow e^{\left|a^{\prime}\right|} a<_{\text {plex }} e^{|a|} a^{\prime},
$$

da kürzere Monome hier automatisch als kleiner eingeordnet werden. Sofern nicht anders vereinbart, wird auf den Monomen über einem gegebenen geordneten Alphabet stets die kanonische Ordnung angenommen.

\section{Definition 1.2.37 (Silbenordnung)}

Seien nun $\left(M,<_{M}\right)$ und $\left(N,<_{N}\right)$ strikt total geordnete Mengen (beispielsweise Monome über einem Alphabet mit einer der obigen Ordnungen), und

$$
\mathcal{M}(M, N):=\left\{\mu_{1} \nu_{1} \mu_{2} \nu_{2} \ldots \mu_{n} \nu_{n} \mu_{n+1}: \mu_{j} \in M, \nu_{j} \in N, n \in \mathbb{N}_{0}\right\}
$$


die Menge der Worte mit Silben abwechselnd aus $M$ und $N$. Auf dieser Menge sind die Striktordnungen

$$
\begin{aligned}
& \mu_{1} \nu_{1} \ldots \mu_{n+1}<_{M^{\prime}} \mu_{1}^{\prime} \nu_{1}^{\prime} \ldots \mu_{m+1}^{\prime}: \Leftrightarrow \mu_{1} \mu_{2} \ldots \mu_{n+1}<_{\operatorname{kan}(M)} \mu_{1}^{\prime} \mu_{2}^{\prime} \ldots \mu_{m+1}^{\prime} \\
& \mu_{1} \nu_{1} \ldots \mu_{n+1}<_{N^{\prime}} \mu_{1}^{\prime} \nu_{1}^{\prime} \ldots \mu_{m+1}^{\prime}: \Leftrightarrow \nu_{1} \nu_{2} \ldots \nu_{n}<_{\operatorname{kan}(N)} \nu_{1}^{\prime} \nu_{2}^{\prime} \ldots \nu_{m}^{\prime}
\end{aligned}
$$

durch die Projektionen von $\mathcal{M}(M, N)$ auf die Monome über $M$ und $N$ definiert. Besteht $N$ nur aus einem Element $c \in \mathcal{A}$, und ist $M$ seinerseits die Menge der Monome über $\mathcal{A} \backslash\{c\}$ mit kanonischer Ordnung, so nennen wir die kombinierte Ordnung $<_{N^{\prime}, M^{\prime}}$ auf der Menge $\mathcal{M}(M, N)$ der Monome über $\mathcal{A}$ Silbenordnung zum Trennbuchstaben $c$. Allgemein, wenn $N=\mathcal{B} \subset \mathcal{A}$ mit induzierter Ordnung von $\mathcal{A}$ ist, und $M$ die Menge der Monome über $\mathcal{A} \backslash \mathcal{B}$ mit kanonischer Ordnung, dann nennen wir $<_{N^{\prime}, M^{\prime}}$ Silbenordnung zu den Trennbuchstaben $\mathcal{B}$.

Bei der Silbenordnung ist zu beachten, dass die kanonische Ordnung auf zwei getrennten Ebenen stattfindet, die folgenden Beispiele mögen dies erläutern:

\section{Beispiel 1.2.38}

Sei $\mathcal{A}=\left\{a_{1}, a_{2}, a_{3}, b_{1}, b_{2}\right\}$ mit $a_{1}<a_{2}<a_{3}$ und $b_{1}<b_{2}$. Dann gilt für Monome über $\mathcal{A}$ in kanonischer Ordnung:

$$
\begin{aligned}
a_{3} & <a_{1} a_{2}, \text { da das linke Monom kürzer ist, } \\
a_{1} a_{2} & <a_{3} a_{1} \text {, da an der ersten Unterscheidungsstelle gilt: } a_{1}<a_{3} .
\end{aligned}
$$

Ist $\mathcal{B}=\left\{b_{1}, b_{2}\right\} \subset \mathcal{A}$ die Menge der Trennbuchstaben, dann gilt für die Silbenordnung zu den Trennbuchstaben $\mathcal{B}$ :

$$
\begin{aligned}
a_{3} b_{1} a_{3} & <a_{1} b_{2} a_{1} a_{2} & & , \text { da } b_{1}<b_{2}, \\
a_{3} b_{1} a_{3} a_{1} a_{2} a_{1} a_{2} a_{3} a_{1} a_{2} & <a_{3} b_{1} b_{1} & & \text {, da } b_{1}<b_{1} b_{1}, \\
a_{1} b_{1} a_{1} a_{2} b_{2} & <a_{3} b_{2} a_{1} a_{2} b_{1} & & \text {, da } b_{1} b_{2}<b_{2} b_{1}, \\
a_{3} a_{2} a_{3} & <b_{1} & & \text {, da } \emptyset<b_{1}, \\
a_{1} b_{1} a_{3} & <a_{1} b_{1} a_{2} a_{1} & & \text {, da } a_{3}<a_{2} a_{1}, \\
a_{2} b_{1} a_{3} a_{2} & <a_{1} a_{3} b_{1} & & \text {, da } a_{2}<a_{1} a_{3} .
\end{aligned}
$$

Im letzten Beispiel ist zu beachten, dass $\emptyset$ durchaus ein Monom über $\mathcal{A}$ ist und damit der linke wie der rechte Ausdruck je zwei Silben enthält, der linke $a_{2}$ und $a_{3} a_{2}$, der rechte $a_{1} a_{3}$ und $\emptyset$. Nach Längenordnung auf Ebene der Monome von Monomen sind sie gleich, also $\left|\left(a_{2}\right)\left(a_{3} a_{2}\right)\right|=\left|\left(a_{1} a_{3}\right)(\emptyset)\right|=2$, und es entscheiden die ersten Silben $a_{2}$ und $a_{1} a_{3}$, die ihrerseits Monome über $\mathcal{A}$ sind. Auf dieser unteren Ebene ist dann $a_{2}<a_{1} a_{3}$ wie üblich.

\section{Satz 1.2.39 (Silbenordnung)}

Sei $\mathcal{A}$ noethersch (z.B. endlich) und $\mathcal{B} \subset \mathcal{A}$. Dann ist die Silbenordnung zu den Trennbuchstaben $\mathcal{B}$ auf der Menge $\mathcal{M}_{\mathcal{A}}$ der Monome über $\mathcal{A}$ noethersch und multiplikativ.

Beweis: [Satz 1.2.39] Wir brauchen nur zu zeigen, dass die Projektionsordnungen $<_{M}$ und $<_{N}$ mit $M=\mathcal{A} \backslash \mathcal{B}$ und $N=\mathcal{B}$ noethersch und multiplikativ sind, dann folgt die Behauptung aus den Eigenschaften der kombinierten Ordnung. 
Noethersch: Jede in $<_{M}$ strikt abfallende Folge $\left(a_{j}\right) \subset \mathcal{M}_{\mathcal{A}}$ induziert eine strikt absteigende Folge $\left(a_{j}^{\prime}\right) \subset \mathcal{M}_{M}$ in den Monomen über $M$. Die kanonische Ordnung auf $\mathcal{M}_{M}$ ist aber noethersch, da sie von der kanonischen Ordnung auf $\mathcal{M}_{\mathcal{A}}$ induziert wird. Folglich gibt es keine strikt absteigende Folge in $\mathcal{M}_{M}$, und damit ist $<_{M}$ noethersch. Analog gilt das für $<_{N}$.

Multiplikativität: $\mathrm{Da}<_{\text {kan }}$ multiplikativ ist, gilt für $a \in M$.

$$
\begin{aligned}
& \mu_{1} \nu_{1} \ldots \mu_{n+1}<_{M} \mu_{1}^{\prime} \nu_{1}^{\prime} \ldots \mu_{m+1}^{\prime} \\
& \Leftrightarrow \quad \mu_{1} \ldots \mu_{n+1}<_{\text {kan }} \mu_{1}^{\prime} \ldots \mu_{m+1}^{\prime} \\
& \Rightarrow \quad \mu_{1} \ldots \mu_{n+1} a<_{\text {kan }} \mu_{1}^{\prime} \ldots \mu_{m+1}^{\prime} a \\
& \Leftrightarrow \quad \mu_{1} \nu_{1} \ldots \mu_{n+1} a<_{M} \mu_{1}^{\prime} \nu_{1}^{\prime} \ldots \mu_{m+1}^{\prime} a .
\end{aligned}
$$

Ist $a$ dagegen in $N$, so bleibt die Projektion nach $\mathcal{M}_{M}$ unverändert, und es gilt nach wie vor $\mu_{1} \nu_{1} \ldots \mu_{m+1} a<_{M} \quad \mu_{1} \nu_{1} \ldots \mu_{m+1} a$.

Analog gilt das für Linksmultiplikation und $<_{N}$.

\subsubsection{Konstruktion von $r$}

Sei $\boldsymbol{A}$ eine endlich erzeugte Algebra gegeben durch die Erzeuger $a_{1}, \ldots a_{\boldsymbol{n}_{\boldsymbol{A}}}$ und die Relationen $\left\{s_{1}, \ldots, s_{n_{I_{A}}}\right\}$. Dann existiert eine exakte Sequenz von Vektorräumen:

$$
0 \rightarrow I_{\boldsymbol{A}} \rightarrow \mathcal{F}_{\boldsymbol{n}_{\boldsymbol{A}}} \rightarrow \boldsymbol{A} \rightarrow 0,
$$

wobei $I_{\boldsymbol{A}}$, das durch $\left\{s_{1}, \ldots, s_{n_{I_{\boldsymbol{A}}}}\right\}$ erzeugte Ideal ist.

Sei auf dem Alphabet $\mathcal{A}=\left\{a_{1}, \ldots a_{n}\right\}$ eine Totalordnung gegeben und sei $<_{\mathcal{M}}$ die kanonische Ordnung auf den Monomen über $\mathcal{A}$. Als Partialordnung auf $\widetilde{\mathcal{F}}$ wählen wir die folgende:

\section{Definition 1.2.40 (Ordnung auf $\widetilde{\mathcal{F}}$ )}

Solange die Terme, in denen das jeweils größte Monom vorkommt, für beide zu vergleichenden Elemente übereinstimmen, ignorieren wir diese Terme.

Wenn die größten Monome sich unterscheiden, dann vergleichen wir nach der kanonischen Ordnung $<_{\mathcal{M}}$.

\section{Beispiel 1.2.41}

Seien $w_{1}, w_{2}, w_{3} \in \mathcal{M}$ mit $w_{1}<_{\mathcal{M}} w_{2}<_{\mathcal{M}} w_{3}$ und $\lambda_{1}, \lambda_{2}, \lambda_{3}, \bar{\lambda}_{3} \in \mathbb{K}$ mit $\lambda_{3} \neq \bar{\lambda}_{3}$, dann gilt bezüglich der Ordnung auf $\widetilde{\mathcal{F}}$ :

- $\lambda_{1} w_{1}+{ }^{\prime} \lambda_{2} w_{2}<\lambda_{3} w_{3}$, da $w_{2}<w_{3}$.

- $\lambda_{1} w_{1}+{ }^{\prime} \lambda_{3} w_{3}>\lambda_{3} w_{3}$, da $w_{1}>0$.

- $\lambda_{1} w_{1}+{ }^{\prime} \lambda_{3} w_{3}<\lambda_{2} w_{2}+{ }^{\prime} \lambda_{3} w_{3}$, da $w_{1}<w_{2}$.

- Aber: $\lambda_{1} w_{1}+{ }^{\prime} \bar{\lambda}_{3} w_{3}$ und $\lambda_{2} w_{2}+{ }^{\prime} \lambda_{3} w_{3}$ sind unvergleichbar, da die größten Monome gleich sind, sich die Koeffizienten aber unterscheiden. 
Wir schreiben jede Relation $s=0$ der Algebra so um, dass das jeweils größte Monom $w$ auf der linken Seite und der Rest, eine Summe von Termen, auf der rechten Seite des Gleichheitszeichens steht. Die Gleichung von links nach rechts gelesen ergibt dann eine Ersetzungsregel. Sie ist von der Form:

$$
w \rightarrow \sum_{i=1}^{n} \lambda_{i} w_{i}
$$

Jede Reduktionsregel hat also die Eigenschaft, dass jedes Monom auf der rechten Seite kleiner ist als das auf der linken.

Die Menge dieser Reduktionsregeln bezeichnen wir mit $r$. Sie induzieren eine Menge von Reduktionsregeln auf $\mathcal{F}$ bzw. $\widetilde{\mathcal{F}}$, die wir mit $\mathcal{R}$ bzw. $\widetilde{\mathcal{R}}$ (siehe: 1.2.7 bzw. 1.2.13 bezeichnen.

\section{Definition 1.2.42 (Gröbnerbasis)}

Falls $r$ vollständig ist, nennen wir die Menge $\left\{s_{1}, \ldots, s_{n_{I_{A}}}\right\}$ eine Gröbnerbasis von $I_{\boldsymbol{A}}$ bezüglich $<_{\mathcal{M}}$.

\section{Bemerkung 1.2.43 (lineare Basis)}

Falls $r$ vollständig ist, dann bilden die Wörter in $\mathcal{F}$ die sich nicht reduzieren lassen eine lineare Basis für $\boldsymbol{A}$.

Da $\widetilde{\mathcal{F}}$ eine Rig ist, induzieren die Verknüpfungen für jede Ersetzungsregel eine Vielzahl an weiteren Regeln:

$$
p \cdot w \cdot s+^{\prime} q \longrightarrow{ }_{\widetilde{\mathcal{R}}} \sum_{i=1}^{n}{ }^{\prime} p \cdot \lambda_{i} w_{i} \cdot s+^{\prime} q,
$$

wobei $p, w, w_{i} s \in \mathcal{M}$ und $q \in \widetilde{\mathcal{F}}$. Da wir die Ordnung geeignet gewählt haben, verkleinert auch jede so erzeugte Regel.

\section{Bemerkung 1.2.44 (Wahl von $S$ )}

Wir wollen hier noch einmal besonders darauf hinweisen, dass es zu einer Algebra verschiedene Mengen von Relationen gibt, die sie definieren. Von der Wahl der Relationen hängt es ab, ob das induzierte Reduktionssystem vollständig ist oder nicht.

\subsubsection{Noethersch}

\section{Satz 1.2.45 (Noethersch)}

Die Ordnung auf $\widetilde{\mathcal{F}}$ ist noethersch, und für jedes $(x, y) \in \widetilde{\mathcal{R}}$ gilt $x>y$, insbesondere ist $\widetilde{\mathcal{R}}$ noethersch.

Beweis: [] Da die Ordnung $<_{\mathcal{M}}$ noethersch und multiplikativ ist, ist es auch die induzierte Ordnung auf $\widetilde{\mathcal{F}}$. Dass jede Reduktionsregel verkleinert, folgt direkt aus der Definition (1.2.13).

Satz 1.2.46 (Vollständig)

Falls die Ordnung auf Monomen $<_{\mathcal{M}}$ noethersch und multiplikativ ist und falls $r$ vollständig ist, dann gilt: 
1. Die induzierten Reduktionssysteme $\mathcal{R}$ und $\widetilde{\mathcal{R}}$ auf $\mathcal{F}$ bzw. $\widetilde{\mathcal{F}}$ sind konvergent.

2. Zwei Elemente aus $\mathcal{F}$ haben genau dann dieselbe Normalform, wenn sie durch die kanonische Projektion $\pi_{\boldsymbol{A}}: \mathcal{F} \rightarrow \boldsymbol{A}$ auf dasselbe Element abgebildet werden.

Beweis: []

Zu 1.: Da die Ordnung multiplikativ ist, gilt nach 1.2 .45 , dass auch $\widetilde{\mathcal{R}}$ noethersch ist. Da $r$ vollständig ist, folgt aus 1.2 .24 dass $\widetilde{\mathcal{R}}$ lokal konfluent ist. Mit 1.1 .17 ist $\widetilde{\mathcal{R}}$ total konfluent und nach 1.2.17 ist auch $\mathcal{R}$ total konfluent.

$\mathrm{Zu} 2 .:$ " $\Rightarrow$ "Seien $f, \bar{f} \in \mathcal{F}$ mit derselben Normalform. Zuerst überlegen wir uns, dass $f-\mathcal{N}(f) \in I_{\boldsymbol{A}}$ gilt: Sei $\left(f, f_{1}\right)$ eine beliebige Reduktionsregel in $\mathcal{R}$, die mit $f$ beginnt. Sei $s=0$ die Relation, die die Reduktionsregel $\left(w, \sum_{i}^{n} \lambda_{i} w\right)$ in $r$ erzeugt, die $\left(f, f_{1}\right)$ induziert. Dann ist

$$
f-f_{1}=p\left(w-\sum_{i}^{n} \lambda_{i} w\right) s \in I_{\boldsymbol{A}}
$$

Durch mehrfaches Anwenden sehen wir, dass auch $f-\mathcal{N}(f) \in I_{\boldsymbol{A}}$.

Für die Differenz von $f$ und $\bar{f}$ gilt dann also:

$$
f-\bar{f}=f-\mathcal{N}(f)+(\mathcal{N}(\bar{f})-\bar{f}) \in I_{A}
$$

somit werden sie auf dasselbe Element in $\boldsymbol{A}$ projiziert.

“ $\Leftarrow$ "Sei $\pi_{\boldsymbol{A}}(f)=\pi_{\boldsymbol{A}}(\bar{f})$, dann gilt:

$$
\begin{aligned}
f-\bar{f} \in I_{\boldsymbol{A}} & \Rightarrow f-\bar{f}=\sum_{k=1}^{m} x_{i_{k}} s_{i_{k}} y_{i_{k}} \\
& \Rightarrow f \text { und } \bar{f} \text { sind Church-Rosser-äquivalent bezüglich } \mathcal{R} \\
& \stackrel{1.1 .15}{\Rightarrow} \mathcal{N}(f)=\mathcal{N}(\bar{f}) .
\end{aligned}
$$

\subsection{Reduktionssystem bezüglich eines Moduls}

Reduktionssysteme kann man auch für Moduln und für Modulabbildungen nutzen, vgl. [Ber78b]. Wir wollen in diesem Kapitel aber keine neue Theorie entwickeln, sondern einen Modul als Teilmenge einer Algebra beschreiben. Genauer, wir beschreiben $\boldsymbol{M}$ als eine Untermenge der freien Algebra $\mathcal{F}_{\boldsymbol{n}_{A}+\boldsymbol{n}_{M}}$, damit wir die in 1.2 beschriebenen Methoden für ein Reduktionssystem für Algebren nutzen können. 


\subsection{1 $\mathcal{P}$-Vollständigkeit}

In 1.2.24 hatten wir gesehen, dass wir Konfluenz nachweisen können, indem wir die minimalen Überschneidungen untersuchen. Nun werden wir zeigen, dass wir nicht alle minimalen Überschneidungen betrachten müssen.

Definition 1.3.1 ( $\mathcal{R}$-Prädikat $\mathcal{P}$ )

Sei $\mathcal{P}: \mathcal{F} \rightarrow\{$ wahr, falsch $\}$ ein Prädikat, das folgende Bedingung erfüllt:

- Falls $(x, y) \in \mathcal{R}$ und $\mathcal{P}(x)=$ wahr, dann ist auch $\mathcal{P}(y)=$ wahr.

Ein solches Prädikat nennen wir $\mathcal{R}$-Prädikat.

Definition 1.3.2 $\left(\mathcal{R}_{\mathcal{P}}\right)$

Für ein $\mathcal{R}$-Prädikat $\mathcal{P}$ bezeichnen wir die Regeln aus $\mathcal{R}$, die mit einem $x$ mit $\mathcal{P}(x)=$ wahr beginnen, mit $\mathcal{R}_{\mathcal{P}}$, also

$$
\mathcal{R}_{\mathcal{P}}:=\{(x, y) \in \mathcal{R} \mid \mathcal{P}(x)=\mathbf{w a h r}\} .
$$

Die Untermenge in $\mathcal{E}$, die Urbild von wahr unter $\mathcal{P}$ ist, bezeichnen wir mit $\mathcal{E}_{\mathcal{P}}$, also

$$
\mathcal{E}_{\mathcal{P}}:=\{x \in \mathcal{E} \mid \mathcal{P}(x)=\mathbf{w a h r}\} .
$$

\section{Bemerkung 1.3.3 (Folgerung)}

Sei $\mathcal{P}$ ein $\mathcal{R}$-Prädikat. Folgende Eigenschaften ergeben sich für $\mathcal{R}_{\mathcal{P}}$ direkt aus der Definition:

- Es gilt:

$$
\mathcal{R}_{\mathcal{P}}=\{(x, y) \in \mathcal{R} \mid \mathcal{P}(x)=\mathbf{w a h r} \text { und } \mathcal{P}(y)=\mathbf{w a h r}\}
$$

- Sei $y_{1} \quad \cdots \cdots \rightarrow_{\mathcal{R}} y_{n}$ ein Reduktionsweg in $\mathcal{R}$ und sei $\mathcal{P}\left(y_{1}\right)=$ wahr, dann gilt für jedes $i=1 \ldots n$ :

$$
\mathcal{P}\left(y_{i}\right)=\mathbf{w a h r}
$$

- Sei $y_{1} \quad \underset{\mathcal{R}}{y_{n}}$ ein Reduktionsweg in $\mathcal{R}$ und sei $\mathcal{P}\left(y_{n}\right)=$ falsch, dann gilt für jedes $i=1 \ldots n$ :

$$
\mathcal{P}\left(y_{i}\right)=\text { falsch }
$$

- Ist $\mathcal{R}$ konvergent, dann ist auch die Teilmenge $\mathcal{R}_{\mathcal{P}}$ auf $\mathcal{E}_{\mathcal{P}}$ ein konvergentes Reduktionssystem.

\section{Definition 1.3.4 (Silbenverträglichkeit)}

Ein Prädikat $\mathcal{P}: \widetilde{\mathcal{F}} \rightarrow\{$ wahr, falsch $\}$ heißt silbenverträglich, falls für jedes $p, s \in \mathcal{M}$ folgende zwei Axiome erfüllt sind:

- Summandenverträglichkeit:

$$
\mathcal{P}\left(p+{ }^{\prime} s\right)=\mathbf{w a h r} \Leftrightarrow \mathcal{P}(p)=\mathbf{w a h r}=\mathcal{P}(s) .
$$


- Faktorenverträglichkeit:

$$
\mathcal{P}(p s)=\mathbf{w a h r} \Rightarrow \mathcal{P}(p)=\mathbf{w a h r}=\mathcal{P}(s) .
$$

\section{Bemerkung 1.3.5 (Alternative Definition)}

Alternativ könnten wir Silbenverträglichkeit auch definieren durch das Axiom.

Sei $q:=\sum_{w \in \mathcal{M}}{ }^{\prime} \sum_{i=1}^{N_{w}} ' \lambda_{w, i} w$, dann gilt:

$$
\mathcal{P}(q)=\mathbf{w a h r} \Rightarrow \mathcal{P}(w)=\mathbf{w a h r} \text { für alle } w \text { mit } N_{w}>0 .
$$

$\operatorname{Satz}$ 1.3.6 $\left(\underset{\overrightarrow{\mathcal{R}}_{\mathcal{P}}}{\longrightarrow} \quad \overrightarrow{\widetilde{\mathcal{R}}}_{\mathcal{P}}\right)$

Sei $\mathcal{P}$ ein silbenverträgliches $\widetilde{\mathcal{R}}$-Prädikat, dann induziert jede Regel aus $\mathcal{R}_{\mathcal{P}}$ einen Weg in $\widetilde{\mathcal{R}}_{\mathcal{P}}$.

Beweis: [Satz 1.3.6]

Sei $\left(g_{1}, g_{2}\right) \in \mathcal{R}_{\mathcal{P}}$, dann gibt es $(x, y) \in r$ mit:

$$
g_{1}=\lambda p x s+q \text { und } g_{2}=\lambda p(y) s+q .
$$

Mit Lemma 1.2.16 lässt sich $\left(g_{1}, g_{2}\right)$ als Weg in $\widetilde{\mathcal{R}}$ schreiben:

$$
\lambda p x s+{ }^{\prime} q \quad \underset{\widetilde{\mathcal{R}}}{>} \lambda p(y) s+^{\prime} q .
$$

Nach Voraussetzung ist $\mathcal{P}\left(\lambda p x s+{ }^{\prime} q\right)=$ wahr und mit Bemerkung 1.3.3 liegt dann der gesamte Weg in $\widetilde{\mathcal{R}}_{\mathcal{P}}$.

Satz 1.3.7 (Konvergenz)

Sei $\mathcal{P}$ ein silbenverträgliches $\widetilde{\mathcal{R}}$-Prädikat. Sei $\widetilde{\mathcal{R}}_{\mathcal{P}}$ konvergent, dann ist auch $\mathcal{R}_{\mathcal{P}}$ konvergent.

Beweis: [Satz 1.3.7] Durch mehrfaches Anwenden von Satz 1.3.6 folgt, dass alle Wege aus $\mathcal{R}_{\mathcal{P}}$ einen Weg in $\widetilde{\mathcal{R}}_{\mathcal{P}}$ bilden. Nun ist dieser Beweis nach Ersetzung von $\mathcal{F}, \widetilde{\mathcal{F}}, \mathcal{R}, \widetilde{\mathcal{R}}$ durch $\mathcal{F}_{P}, \widetilde{\mathcal{F}}_{P}, \mathcal{R}_{P}, \widetilde{\mathcal{R}}_{P}$ identisch mit dem für Satz 1.2 .17

\section{Definition 1.3.8 ( $\mathcal{P}$-minimale Überschneidung)}

Sei $\mathcal{P}$ ein silbenverträgliches $\widetilde{\mathcal{R}}$-Prädikat. Eine minimale Überschneidung $(w, V, \bar{V})$ mit $\mathcal{P}(w)=$ wahr, heißt $\mathcal{P}$-minimale Überschneidung.

\section{Definition 1.3.9 ( $\mathcal{P}$-vollständig)}

Ein Wortersetzungssystem $r$ heißt $\mathcal{P}$-vollständig, falls jede $\mathcal{P}$-minimale Überschneidung behebbar bezüglich $\widetilde{\mathcal{R}}$ ist.

Satz 1.3.10 ( $\mathcal{P}$-vollständig $\Rightarrow$ Konfluenz)

Sei r ein $\mathcal{P}$-vollständiges Wortersetzungssystem, dann ist $\left(\widetilde{\mathcal{F}}_{\mathcal{P}}, \widetilde{\mathcal{R}}_{\mathcal{P}}\right)$ ein lokal konfluentes Reduktionssystem.

Beweis: [Satz 1.3.10

Sei $f \in \widetilde{\mathcal{F}}_{\mathcal{P}}$ und $\left(f, g_{1}\right),\left(f, g_{2}\right) \in \widetilde{\mathcal{R}}_{\mathcal{P}}$, dann gilt auch $\left(f, g_{1}\right),\left(f, g_{2}\right) \in \widetilde{\mathcal{R}}$. Falls 
$\left(f, g_{1}\right),\left(f, g_{2}\right) \notin$ Rel gilt, dann liegt wenigstens einer von beiden in Rel. Der Beweis ist nun genauso wie der für Satz 1.2.24, nur dass wir anstelle von Bemerkung 1.2.18 den Satz 1.3.6 nutzen.

Falls $\left(f, g_{1}\right),\left(f, g_{2}\right) \in$ Rel gilt, dann lassen sich $f$ und $g_{i}$ schreiben als:

$$
\begin{aligned}
f & =\lambda_{1} p_{1} x_{1} s_{1}+^{\prime} q_{1}=\lambda_{2} p_{2} x_{2} s_{2}+^{\prime} q_{2}, \\
g_{i} & =\lambda_{i} p_{i}\left(y_{i}\right) s_{i}+^{\prime} q_{i},
\end{aligned}
$$

wobei $\lambda_{i} \in \mathbb{K}^{*}$ und $\left(x_{i}, y_{i}\right) \in r$, sowie $p_{i}, s_{i} \in \mathcal{M}$ und $q_{i} \in \widetilde{\mathcal{F}}$.

Falls $q_{1} \neq q_{2}$, dann muss $q_{1}=\lambda_{2} p_{2} x_{2} s_{2}$ und $q_{2}=\lambda_{1} p_{1} x_{1} s_{1}$ sein. In diesem Fall lassen sich $g_{1}$ und $g_{2}$ zu $\lambda_{1} p_{1} x_{1} s_{1}+{ }^{\prime} \lambda_{2} p_{2} x_{2} s_{2}$ reduzieren.

Sei also $q_{1}=q_{2}$.

Falls $\lambda_{1} p_{1} x_{1} s_{1}=\lambda_{2} p_{2} x_{2} s_{2}$, dann ist der Beweis genauso wie der für Satz 1.2.24

Falls $\lambda_{1} p_{1} x_{1} s_{1} \neq \lambda_{2} p_{2} x_{2} s_{2}$, dann gibt es folgende Möglichkeiten:

1. getrennte Silben: Es gibt ein $\boldsymbol{w} \in \mathcal{M}$ mit:

$$
p_{1} x_{1} s_{1}=p_{1} x_{1} \boldsymbol{w} x_{2} s_{2}=p_{2} x_{2} s_{2} .
$$

2. getrennte Silben vertauscht: Es gibt ein $\boldsymbol{w} \in \mathcal{M}$ mit:

$$
p_{1} x_{1} s_{1}=p_{2} x_{2} \boldsymbol{w} x_{1} s_{1}=p_{2} x_{2} s_{2} .
$$

3. volle Überschneidung: Es gibt $\boldsymbol{w}_{\mathbf{1}}, \boldsymbol{w}_{\mathbf{2}} \in \mathcal{M}$ mit:

$$
x_{1}=\boldsymbol{w}_{\mathbf{1}} x_{2} \boldsymbol{w}_{\mathbf{2}}
$$

4. volle Überschneidung vertauscht: Es gibt $\boldsymbol{w}_{\mathbf{1}}, \boldsymbol{w}_{\mathbf{2}} \in \mathcal{M}$ mit:

$$
\boldsymbol{w}_{1} x_{1} \boldsymbol{w}_{2}=x_{2}
$$

5. Teil-Überschneidung: Es gibt $\boldsymbol{w}_{1}, \boldsymbol{w}_{\mathbf{2}} \in \mathcal{M}$ mit:

$$
\boldsymbol{w}_{\mathbf{1}} x_{1}=x_{2} \boldsymbol{w}_{\mathbf{2}}
$$

6. Teil-Überschneidung vertauscht: Es gibt $\boldsymbol{w}_{1}, \boldsymbol{w}_{2} \in \mathcal{M}$ mit:

$$
x_{1} \boldsymbol{w}_{\mathbf{2}}=\boldsymbol{w}_{\mathbf{1}} x_{2}
$$


Die Fälle 1 und2können wir wieder wie in Satz 1.2.24 behandeln.

Die Fälle 3 und 4 sind symmetrisch, wir zeigen 3

Hier gilt $\mathcal{P}(f)=$ wahr. Da $f=p_{1} x_{1} s_{1}+^{\prime} q$ gilt, ist wegen der Silbenverträglichkeit von $\mathcal{P}$ auch $\mathcal{P}\left(p_{1} x_{1} s_{1}\right)=$ wahr, also ist auch $\mathcal{P}\left(x_{1}\right)=$ wahr.

Demnach ist die minimale Überschneidung $\left(x_{1}=\boldsymbol{w}_{\mathbf{1}} x_{2} \boldsymbol{w}_{\mathbf{2}},\left(x_{1}, y_{1}\right),\left(x_{2}, y_{2}\right)\right)$ eine $\mathcal{P}$-minimale Überschneidung. Nach Voraussetzung lässt sie sich beheben. Es gibt also Reduktionswege:

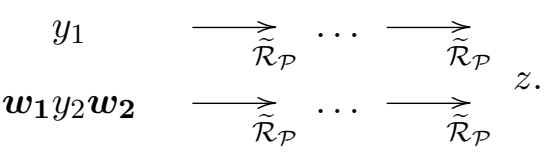

Wegen Satz1.3.6 gibt es dann auch Reduktionswege:

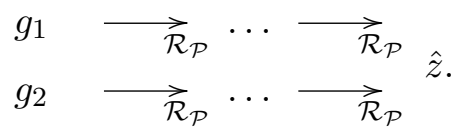

Die Fälle 5 und 6 sind symmetrisch, wir zeigen 5 .

Hier ist $\boldsymbol{w}_{\mathbf{1}}$ ein Teilwort aus $p_{1}$ und $\mathcal{P}(f)=\mathbf{w a h r}$, also ist auch $\mathcal{P}\left(p_{1} x_{1} s_{1}\right)=\mathbf{w a h r}$ und auch $\mathcal{P}\left(\boldsymbol{w}_{\mathbf{1}} x_{1}\right)=$ wahr.

Demnach ist die minimale Überschneidung $\left(\boldsymbol{w}_{\mathbf{1}} x_{1}=x_{2} \boldsymbol{w}_{\mathbf{2}},\left(x_{1}, y_{1}\right),\left(x_{2}, y_{2}\right)\right)$ eine $\mathcal{P}$-minimale Überschneidung. Nach Voraussetzung lässt sie sich beheben. Es gibt also Reduktionswege:

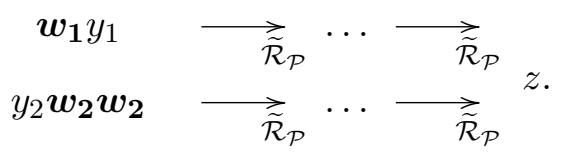

Wegen Satz1.3.6 gibt es dann auch Reduktionswege:

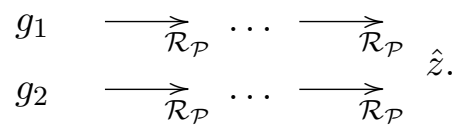

Es lassen sich also alle Überschneidungen beheben, somit ist $\widetilde{\mathcal{R}}_{\mathcal{P}}$ lokal konfluent.

\subsection{2 $A \otimes A^{o p}$-Moduln}

Wie in den vorhergehenden Abschnitten sei $\mathcal{A}$ das Alphabet mit den Buchstaben $a_{1}, \ldots, a_{\boldsymbol{n}_{\boldsymbol{A}}}$ und sei $\mathcal{M}$ die Menge der Monome über $\mathcal{A}$, weiter sei $\boldsymbol{A}$ eine Algebra und $\mathcal{F}_{\boldsymbol{n}_{\boldsymbol{A}}}$ die freie Algebra mit Erzeugern aus $\mathcal{A}$. Bezüglich $\boldsymbol{A}$ sei $r_{\boldsymbol{A}}$ ein vollständiges Reduktionssystem.

Nun sei $\boldsymbol{M}$ ein durch $\boldsymbol{e}_{1}, \ldots, \boldsymbol{e}_{\boldsymbol{n}_{M}}$ endlich erzeugter $\boldsymbol{A} \otimes \boldsymbol{A}^{o p}$-Modul. Mit $\mathcal{M}_{\boldsymbol{A}, \boldsymbol{M}}$ bezeichnen wir die Menge der Monome über dem Alphabet mit den Buchstaben $\mathcal{A} \cup$ $\left\{\boldsymbol{\epsilon}_{1}, \ldots, \boldsymbol{\epsilon}_{\boldsymbol{n}_{M}}\right\}$. Sei $\mathcal{F}_{\boldsymbol{n}_{\boldsymbol{A}}+\boldsymbol{n}_{M}}$ die freie Algebra über $\mathcal{M}_{\boldsymbol{A}, \boldsymbol{M}}$ mit der Silbenordnung zu den Trennungsbuchstaben $\left\{\boldsymbol{\epsilon}_{1}, \ldots, \boldsymbol{\epsilon}_{\boldsymbol{n}_{M}}\right\}$.

Nehmen wir an, dass es $\boldsymbol{n}_{\overline{\boldsymbol{M}}} \in \mathbb{N}$ und $S:=\left\{\overline{\boldsymbol{e}}_{1}, \ldots, \overline{\boldsymbol{e}}_{\boldsymbol{n}_{\bar{M}}}\right\}$ gibt, so dass folgende Sequenz exakt ist:

$$
0 \rightarrow \overline{\boldsymbol{M}} \hookrightarrow\left(\boldsymbol{A} \otimes \boldsymbol{A}^{o p}\right)^{\boldsymbol{n}_{M}} \stackrel{\varpi_{M}}{\rightarrow} \boldsymbol{M} \rightarrow 0
$$


wobei $\overline{\boldsymbol{M}}$ der durch durch $\mathrm{S}$ erzeugte $\boldsymbol{A} \otimes \boldsymbol{A}^{o p}$-Modul ist.

Ein Element $c \in\left(\boldsymbol{A} \otimes \boldsymbol{A}^{o p}\right)^{\boldsymbol{n}_{M}}$ schreiben wir als

$$
c=\sum_{\sigma=1}^{N} p_{\sigma} \boldsymbol{\epsilon}_{i_{\sigma}} s_{\sigma}, \text { wobei } p_{\sigma}, s_{\sigma} \in \boldsymbol{A} \text { und } \boldsymbol{\epsilon}_{i_{\sigma}} \text { Erzeuger von } \boldsymbol{M} \text { sind. }
$$

Da $\overline{\boldsymbol{M}} \hookrightarrow\left(\boldsymbol{A} \otimes \boldsymbol{A}^{o p}\right)^{\boldsymbol{n}_{M}}$ injektiv ist, können wir jeden Erzeuger $\overline{\boldsymbol{e}}_{\iota}$ von $\overline{\boldsymbol{M}}$ mit seinem Bild identifizieren. Es gibt also $\iota_{\sigma} \in\left\{1 \ldots \boldsymbol{n}_{\boldsymbol{M}}\right\}$ und $\bar{p}_{\sigma}, \bar{s}_{\sigma} \in \boldsymbol{A}$, so dass

$$
\bar{e}_{\iota}=\sum_{\sigma=1}^{\bar{n}_{\iota}} \bar{p}_{\sigma} \epsilon_{\iota \sigma} \bar{s}_{\sigma}
$$

Für jedes $\bar{p}_{\sigma}, \bar{s}_{\sigma}$ wählen wir ein Urbild unter der kanonischen Projektion $\pi_{\boldsymbol{A}}: \mathcal{F}_{\boldsymbol{n}_{\boldsymbol{A}}} \rightarrow$ $\boldsymbol{A}$ und erhalten so ein Element in $\left(\mathcal{F}_{\boldsymbol{n}_{\boldsymbol{A}}} \otimes \mathcal{F}_{\boldsymbol{n}_{\boldsymbol{A}}}\right)^{\boldsymbol{n}_{M}} \subset \mathcal{F}_{\boldsymbol{n}_{\boldsymbol{A}}+\boldsymbol{n}_{M}}$ :

$$
\tilde{e}_{\iota}=\sum_{\sigma=1}^{\bar{n}_{\iota}} \tilde{p}_{\sigma} \epsilon_{\iota_{\sigma}} \tilde{s}_{\sigma}
$$

Aus den Erzeugern von $\overline{\boldsymbol{M}}$ werden wir nun ein Reduktionssystem $r_{\boldsymbol{M}} \subset \mathcal{M}_{\boldsymbol{A}, \boldsymbol{M}} \times$ $\mathcal{F}_{\boldsymbol{n}_{\boldsymbol{A}}+\boldsymbol{n}_{M}}$ konstruieren. Dazu stellen wir die obige Summe so um, dass der größte Summand, wir bezeichnen ihn mit $x_{\iota}$, ohne seinen Koeffizienten $\lambda_{\iota}$ auf der linken Seite steht:

$$
r_{\boldsymbol{M}}:=\left\{\left(x_{\iota}, x_{\iota}-\lambda_{\iota}^{-1} \tilde{e}_{\iota}\right) \mid \iota=1 \ldots \boldsymbol{n}_{\overline{\boldsymbol{M}}}\right\} .
$$

Um zu verdeutlichen, dass dieses Wortersetzungssystem vom Erzeugersystem $S$ des Moduls $\bar{M}$ abhängt, nennen wir $r_{M}$ auch das durch $S$ induzierte Reduktionssystem. Die Reduktionsregeln in $r_{M}$ beginnen jeweils mit einem Wort, in dem genau ein Erzeuger des Moduls vorkommt.

\section{Bemerkung 1.3.11 (Wahl des Erzeugersystems)}

Wenn man das Erzeugersystem $S$ für $\overline{\boldsymbol{M}}$ geeignet gewählt hat, wird das induzierte Reduktionssystem $r_{\boldsymbol{A}, Q}$ vollständig. Diese Wahl ist oft jedoch sehr schwer. Es gibt Algorithmen, die aus einem gegebenen Erzeugersystem ein neues Erzeugersystem $S^{\prime}$ konstruieren, so dass das induzierte Wortersetzungssystem vollständig ist. Leider terminieren diese Algorithmen nicht in jedem Fall.

Zur Erinnerung, in den vorhergehenden Kapiteln haben wir ein Wortersetzungssystem $r_{\boldsymbol{A}}$ bezüglich einer Algebra $\boldsymbol{A}$ konstruiert. Wir haben $r_{\boldsymbol{A}}$ aus einem Erzeugersystem $S_{\boldsymbol{A}}$ für das Ideal $I_{\boldsymbol{A}}$ konstruiert, wobei $I_{\boldsymbol{A}}$ definiert war durch:

$$
0 \rightarrow I_{\boldsymbol{A}} \hookrightarrow \mathcal{F}_{n} \rightarrow \boldsymbol{A} \rightarrow 0 .
$$

Sei $r_{M}$ wie oben konstruiert, dann setzen wir:

$$
r_{\boldsymbol{A}, M}:=r_{\boldsymbol{A}} \cup r_{\boldsymbol{M}} \text {. }
$$

Wir wollen nun ein Prädikat auf $\mathcal{F}_{\boldsymbol{n}_{A}+\boldsymbol{n}_{M}}$ definieren, mit dem wir die Elemente des Moduls markieren können. Dazu betrachten wir zunächst folgende Definition. 
Definition 1.3.12 $(\mathcal{F}(0), \mathcal{F}(1))$

Die Menge der Elemente aus $\mathcal{F}_{\boldsymbol{n}_{\boldsymbol{A}}+\boldsymbol{n}_{M}}$, deren Summanden keinen bzw. genau einen Erzeuger von $\boldsymbol{M}$ enthalten, bezeichnen wir mit $\mathcal{F}(0)$ bzw. $\mathcal{F}(1)$.

Lemma 1.3.13 $(\mathcal{F}(1))$

Die kanonische Projektion $\pi_{M}: \mathcal{F}(1) \rightarrow M$ ist surjektiv.

Beweis: [Lemma 1.3.13] Sei $c \in \boldsymbol{M}$, dann gibt es $\iota_{\sigma} \in\left\{1 \ldots \boldsymbol{n}_{\boldsymbol{M}}\right\}$ und $\bar{p}_{\sigma}, \bar{s}_{\sigma} \in \boldsymbol{A}$ mit:

$$
c=\sum_{\sigma=1}^{N} p_{i_{\sigma}} \boldsymbol{\epsilon}_{i_{\sigma}} s_{i_{\sigma}} .
$$

Da die kanonische Projektion $\pi_{\boldsymbol{A}}: \mathcal{F}_{\boldsymbol{n}_{\boldsymbol{A}}} \rightarrow \boldsymbol{A}$ surjektiv ist, können wir für jedes $p_{i}, s_{i}$ ein Urbild $\tilde{p}_{i}, \tilde{s}_{i} \in \mathcal{F}_{\boldsymbol{n}_{\boldsymbol{A}}}$ wählen. Dann gilt:

$$
\pi_{M}\left(\sum_{\sigma=1}^{N} \tilde{p}_{i_{\sigma}} \boldsymbol{\epsilon}_{i_{\sigma}} \tilde{s}_{i_{\sigma}}\right)=c .
$$

Bemerkung 1.3.14 $\left(\pi_{M}=\varpi_{M} \circ \varpi_{A}\right)$

Mit $\left(\mathcal{F}_{\boldsymbol{n}_{\boldsymbol{A}}} \otimes \mathcal{F}_{\boldsymbol{n}_{\boldsymbol{A}}}\right)^{\boldsymbol{n}_{M}} \subset \mathcal{F}(1) \subset \mathcal{F}_{\boldsymbol{n}_{\boldsymbol{A}}+\boldsymbol{n}_{M}}$ können wir die kanonische Projektion $\pi_{\boldsymbol{M}}: \mathcal{F}(1) \rightarrow \boldsymbol{M}$ als Verknüpfung der folgenden Abbildungen schreiben:

$$
\begin{aligned}
& \varpi_{\boldsymbol{A}}: \mathcal{F}(1) \rightarrow\left(\boldsymbol{A} \otimes \boldsymbol{A}^{o p}\right)^{\boldsymbol{n}_{M}} \text { und } \\
& \varpi_{\boldsymbol{M}}:\left(\boldsymbol{A} \otimes \boldsymbol{A}^{o p}\right)^{\boldsymbol{n}_{M}} \rightarrow \boldsymbol{M} .
\end{aligned}
$$

Lemma 1.3.15 (Zerlegung von $\operatorname{Kern}\left(\varpi_{A}\right)$ )

Sei $I:=\operatorname{Kern}\left(\pi_{\boldsymbol{A}}: \mathcal{F}_{\boldsymbol{n}_{\boldsymbol{A}}} \rightarrow \boldsymbol{A}\right)$, dann lässt sich $\operatorname{Kern}\left(\varpi_{\boldsymbol{A}}\right)$ wie folgt zerlegen:

$$
\operatorname{Kern}\left(\varpi_{\boldsymbol{A}}\right)=\sum_{i=1}^{\boldsymbol{n}_{M}} I \epsilon_{i} \mathcal{F}_{\boldsymbol{n}_{\boldsymbol{A}}}+\sum_{i=1}^{\boldsymbol{n}_{\boldsymbol{M}}} \mathcal{F}_{\boldsymbol{n}_{\boldsymbol{A}}} \epsilon_{i} I .
$$

Beweis: [Lemma 1.3.15] Wir zeigen zunächst ,つ“"

Alle Summanden liegen schon im Kern, da

$$
\varpi_{\boldsymbol{A}}\left(I \epsilon_{i} \mathcal{F}_{\boldsymbol{n}_{\boldsymbol{A}}}\right)=\varpi_{\boldsymbol{A}}(I) \epsilon_{i} \varpi_{\boldsymbol{A}}\left(\mathcal{F}_{\boldsymbol{n}_{\boldsymbol{A}}}\right)=0
$$

und auch

$$
\varpi_{\boldsymbol{A}}\left(\mathcal{F}_{\boldsymbol{n}_{\boldsymbol{A}}} \epsilon_{i} I\right)=\varpi_{\boldsymbol{A}}\left(\mathcal{F}_{\boldsymbol{n}_{\boldsymbol{A}}}\right) \epsilon_{i} \varpi_{\boldsymbol{A}}(I)=0 .
$$

Als nächstes zeigen wir „ৎ““:

Sei

$$
f=\sum_{i=1}^{n_{M}} \sum_{j=1}^{m_{i}} \lambda_{i, j} p_{i, j} \epsilon_{i} s_{i, j} \in \operatorname{Kern}\left(\varpi_{\boldsymbol{A}}\right) \subset \mathcal{F}(1),
$$

wobei $\lambda_{i, j} \in \mathbb{K}$ und $p_{i, j}, s_{i, j} \in \mathcal{M}$.

Es gilt also:

$$
0=\varpi_{\boldsymbol{A}}(f)=\sum_{i=1}^{n_{M}} \sum_{j=1}^{m_{i}} \lambda_{i, j} \pi_{\boldsymbol{A}}\left(p_{i, j}\right) \epsilon_{i} \pi_{\boldsymbol{A}}\left(s_{i, j}\right) .
$$


Nun ist $\left\{\epsilon_{1}, \ldots, \epsilon_{\boldsymbol{n}_{M}}\right\}$ eine Basis des freien $\boldsymbol{A}$-Bimoduls $\left(\boldsymbol{A} \otimes \boldsymbol{A}^{o p}\right)^{\boldsymbol{n}_{M}}$, also ist für jedes $i=1, \ldots \boldsymbol{n}_{\boldsymbol{M}}$ :

$$
0=\sum_{j=1}^{m_{i}} \pi_{\boldsymbol{A}}\left(p_{i, j}\right) \otimes \pi_{\boldsymbol{A}}\left(s_{i, j}\right)
$$

Anders gesagt, ist

$$
\sum_{j=1}^{m_{i}}\left(p_{i, j}\right) \otimes\left(s_{i, j}\right) \in \operatorname{Kern}\left(\pi_{\boldsymbol{A}} \otimes \pi_{\boldsymbol{A}}: \mathcal{F}_{\boldsymbol{n}_{\boldsymbol{A}}} \otimes \mathcal{F}_{\boldsymbol{n}_{\boldsymbol{A}}} \rightarrow \boldsymbol{A} \otimes \boldsymbol{A}\right) .
$$

Für diesen Kern gilt jedoch:

$$
\operatorname{Kern}\left(\pi_{\boldsymbol{A}} \otimes \pi_{\boldsymbol{A}}: \mathcal{F}_{\boldsymbol{n}_{\boldsymbol{A}}} \otimes \mathcal{F}_{\boldsymbol{n}_{\boldsymbol{A}}} \rightarrow \boldsymbol{A} \otimes \boldsymbol{A}\right)=I \otimes \mathcal{F}_{\boldsymbol{n}_{\boldsymbol{A}}}+\mathcal{F}_{\boldsymbol{n}_{\boldsymbol{A}}} \otimes I .
$$

Also ist

$$
\varpi_{\boldsymbol{A}}(f) \subset \sum_{i=1}^{\boldsymbol{n}_{M}}\left(I \epsilon_{i} \mathcal{F}_{\boldsymbol{n}_{\boldsymbol{A}}}+\mathcal{F}_{\boldsymbol{n}_{\boldsymbol{A}}} \epsilon_{i} I\right)
$$

Satz 1.3.16 (Normalform)

Sei $r_{A, M}$ wie oben konstruiert und sei $\mathcal{R}$ durch $r_{\boldsymbol{A}, M}$ induziert. Seien $f, \bar{f} \in \mathcal{F}(1)$, dann sind die Aussagen

1. $\pi_{M}(f)=\pi_{M}(\bar{f})$

2. $f<\ldots, \bar{R}$

äquivalent. Also stimmen, falls $\mathcal{R}$ noethersch ist, die Menge der Normalformen und das Bild von $\pi_{M}$ überein.

Beweis: [Satz 1.3.16]

$1 \Rightarrow 2$ :

Sei $\pi_{\boldsymbol{M}}(f)=\pi_{\boldsymbol{M}}(\bar{f})$, also ist $\pi_{\boldsymbol{M}}(f-\bar{f})=0$ und $\varpi_{\boldsymbol{A}}(f-\bar{f}) \in \operatorname{Kern}\left(\varpi_{\boldsymbol{M}}\right)=\overline{\boldsymbol{M}}$. Es gibt also $\iota_{\sigma} \in\left\{1 \ldots \boldsymbol{n}_{\boldsymbol{M}}\right\}$ und $\bar{p}_{\sigma}, \bar{s}_{\sigma} \in \boldsymbol{A}$, so dass

$$
\varpi_{\boldsymbol{A}}(f-\bar{f})=\sum_{\sigma=1}^{n} \bar{p}_{\sigma} \boldsymbol{\epsilon}_{\iota_{\sigma}} \bar{s}_{\sigma} .
$$

Für jedes $\bar{p}_{\sigma}, \bar{s}_{\sigma}$ wählen wir ein Urbild unter der kanonischen Projektion $\pi_{\boldsymbol{A}}: \mathcal{F}_{\boldsymbol{n}_{\boldsymbol{A}}} \rightarrow$ $\boldsymbol{A}$ und erhalten so ein Urbild unter $\varpi_{\boldsymbol{A}}$ :

$$
\sum_{\sigma=1}^{n} \bar{p}_{\sigma} \boldsymbol{\epsilon}_{\iota_{\sigma}} \bar{s}_{\sigma}=\varpi_{\boldsymbol{A}}\left(\sum_{\sigma=1}^{n} \tilde{p}_{\sigma} \boldsymbol{\epsilon}_{\iota_{\sigma}} \tilde{s}_{\sigma}\right) .
$$

Nun gilt mit Lemma1.3.15:

$$
\underbrace{f-\bar{f}-\sum_{\sigma=1}^{n} \tilde{p}_{\sigma} \boldsymbol{\epsilon}_{\iota_{\sigma}} \tilde{s}_{\sigma}}_{\in \operatorname{Kern}\left(\varpi_{\boldsymbol{A}}\right)}=\underbrace{g_{1}}_{\in \sum I \epsilon_{i} \mathcal{F}_{n_{\boldsymbol{A}}}}+\underbrace{g_{2}}_{\sum \mathcal{F}_{\boldsymbol{n}_{\boldsymbol{A}}} \epsilon_{i} I}
$$


Der Anteil in $I$ für jeden Summanden aus $g_{1}$ und $g_{2}$ lässt sich schreiben als $p(x-y) s$, wobei $p, s \in \mathcal{F}_{\boldsymbol{n}_{\boldsymbol{A}}}$ und $(x, y) \in r_{\boldsymbol{A}}$; ähnlich gilt $\epsilon_{i}=\left(x_{i}, y_{i}\right)$ mit $\left(x_{i}, y_{i}\right) \in r_{\boldsymbol{M}}$. Zusammen sehen wir, dass $f<\kappa_{\mathcal{R}}>\bar{f}$.

$2 \Rightarrow 1$ : Sei $g_{1}<{ }_{\mathcal{R}}>g_{n}$, dann gibt es eine endliche Folge $\left(g_{i}, g_{i+1}\right)$, wobei $\left(g_{i}, g_{i+1}\right) \in$ $\mathcal{R}$ oder $\left(g_{i+1}, g_{i}\right) \in \mathcal{R}$. Für die Behauptung reicht es also zu zeigen: Wenn $g, \bar{g} \in \mathcal{R}$ ist, dann gilt auch $\pi_{\boldsymbol{M}}(g)=\pi_{\boldsymbol{M}}(\bar{g})$.

Eine Regel $(g, \bar{g}) \in \mathcal{R})$ lässt sich schreiben als:

$$
(\lambda p x s+q, \lambda p y s+q),
$$

wobei $p, s \in \mathcal{M}_{\boldsymbol{A}, \boldsymbol{M}}, q \in \mathcal{F}_{\boldsymbol{n}_{\boldsymbol{A}}+\boldsymbol{n}_{\boldsymbol{M}}}$ und $(x, y) \in r_{\boldsymbol{A}} \cup r_{\boldsymbol{M}}$.

Also gilt: $g-\bar{g}=p(x-y) s$ und somit auch

$$
\varpi_{\boldsymbol{A}}(g-\bar{g})=\varpi_{\boldsymbol{A}}(p(x-y) s)=\varpi_{\boldsymbol{A}}(p) \varpi_{\boldsymbol{A}}(x-y) \varpi_{\boldsymbol{A}}(s) .
$$

Wir betrachten folgende zwei Fälle:

- Falls $(x, y) \in r_{\boldsymbol{A}}$, dann gilt $\varpi_{\boldsymbol{A}}(x-y)=0$. Dann ist schon $\varpi_{\boldsymbol{A}}(g-\bar{g})=0$.

- Falls $(x, y) \in r_{M}$, dann gilt $x-y=\lambda^{-1} \tilde{e}$, wobei $\tilde{e}$ wie zu Beginn des Abschnitts aus einem Erzeuger $\bar{e}$ von $\bar{M}$ konstruiert ist. Es folgt $\varpi_{\boldsymbol{A}}(x-y)=\bar{e} \in$ $\overline{\boldsymbol{M}}$, also $\varpi_{M}(\bar{e})=0$.

Da $\pi_{M}=\varpi_{M} \circ \varpi_{A}$, folgt in beiden Fällen $\pi_{M}(g)=\pi_{M}(\bar{g})$.

Definition 1.3.17 (Modul Prädikat $\mathcal{P}_{M}$ )

Sei $\widetilde{\mathcal{R}}$ das Reduktionssystem auf $\mathcal{F}_{\boldsymbol{n}_{\boldsymbol{A}}+\boldsymbol{n}_{M}}$, das durch

$$
r_{\boldsymbol{A}, M}:=r_{\boldsymbol{A}} \cup r_{M}
$$

induziert ist. Das Modul Prädikat $\mathcal{P}_{\boldsymbol{M}}$ ist folgendermaßen definiert. Sei $c \in \mathcal{F}_{\boldsymbol{n}_{\boldsymbol{A}}+\boldsymbol{n}_{M}}$, dann gilt:

$$
\mathcal{P}_{\boldsymbol{M}}(c)=\mathbf{w a h r} \Leftrightarrow c \in \mathcal{F}(0) \cup \mathcal{F}(1) .
$$

\section{Satz 1.3.18 (Konvergenz)}

Sei die Ordnung auf den Monomen $\mathcal{M}_{A, M}$ so gewählt, dass sie noethersch und multi-

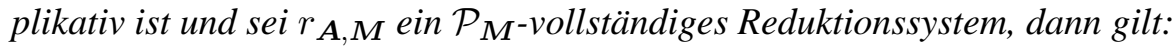

1. Die Reduktionssysteme $\mathcal{R}_{\mathcal{P}_{M}}$ und $\widetilde{\mathcal{R}}_{\mathcal{P}_{M}}$ auf $\mathcal{F}_{\mathcal{P}_{M}}$ bzw. $\widetilde{\mathcal{F}}_{\mathcal{P}_{M}}$ sind konvergent.

2. Zwei Elemente aus $\mathcal{F}(1) \subset \mathcal{F}_{\mathcal{P}_{M}}$ haben genau dann dieselbe Normalform, wenn sie durch die kanonische Projektion $\pi_{M}: \mathcal{F}(1) \rightarrow M$ auf dasselbe Element abgebildet werden.

Beweis: [Satz 1.3.18] Zu 1.: Da die Ordnung multiplikativ ist, folgt aus Satz 1.2.45, dass $\widetilde{\mathcal{R}}_{\mathcal{P}_{M}} \subset \mathcal{R}$ auch noethersch ist. Da $r_{\boldsymbol{A}, M} \mathcal{P}_{M^{-}}$-vollständig ist, folgt aus Satz 
1.3.10, dass auch $\widetilde{\mathcal{R}}_{\mathcal{P}_{M}}$ lokal konfluent ist. Mit Satz 1.1.17 ist $\widetilde{\mathcal{R}}_{\mathcal{P}_{M}}$ total konfluent und nach Satz 1.3 .10 ist auch $\mathcal{R}_{\mathcal{P}_{M}}$ total konfluent.

$\mathrm{Zu} 2$. :

, $\Rightarrow$ “:

Seien $f, \bar{f} \in \mathcal{F}(1)$ mit derselben Normalform, dann gilt auch $f<{ }_{\mathcal{R}}>\bar{f}$ und mit Satz 1.3 .16 gilt

$$
\pi(f)=\pi(\bar{f})
$$

,$\Leftarrow$ ":

Sei $\pi(f)=\pi(\bar{f})$, dann gilt nach Satz 1.3 .16 auch $f \ll \ldots \overline{\mathcal{R}} \bar{f}$. Da $\mathcal{R}$ nach 1 . konvergent ist, stimmen nach Satz 1.1.17 die Normalformen von $f$ und $\bar{f}$ überein.

\section{Definition 1.3.19 (Gröbnerbasis)}

Sei $r_{\boldsymbol{A}, \boldsymbol{M}}$ wie oben konstruiert. Falls $r_{\boldsymbol{A}, \boldsymbol{M}}$ ein $\mathcal{P}_{\boldsymbol{M}}$-vollständiges Reduktionssystem ist, dann heißt $\left\{\tilde{\boldsymbol{e}}_{1}, \ldots, \tilde{\boldsymbol{e}}_{\boldsymbol{n}_{M}}\right\}$ Gröbnerbasis von dem Untermodul $\overline{\boldsymbol{M}} \subset\left(\boldsymbol{A} \otimes \boldsymbol{A}^{o p}\right)^{\boldsymbol{n}_{M}}$.

Um zu zeigen, dass $\mathcal{R}$ konvergent ist, brauchen wir also nur nachzuweisen, dass die minimalen Überschneidungen in $r_{\boldsymbol{A}} \cup r_{\boldsymbol{M}}$ mit höchstens einem Modulerzeuger behebbar sind.

\subsubsection{Kern eines $A \otimes A^{o p}$-Homomorphismus}

Sei $\boldsymbol{A}=\mathcal{F} / I$ eine Algebra und $r_{\boldsymbol{A}} \subset \mathcal{M} \times \mathcal{F}$ ein Reduktionssystem für $I$. Wir wollen nun eine Methode angeben, wie wir den Kern einer Abbildung zwischen freien $\boldsymbol{A} \otimes \boldsymbol{A}^{o p}$-Moduln berechnen können.

Sei $\phi:\left(\boldsymbol{A} \otimes \boldsymbol{A}^{o p}\right)^{n_{1}} \rightarrow\left(\boldsymbol{A} \otimes \boldsymbol{A}^{o p}\right)^{n_{2}}$ ein $\boldsymbol{A} \otimes \boldsymbol{A}^{o p}$-Homomorphismus. Den Graphen der Abbildung bezeichnen wir mit

$$
\Gamma(\phi):=\left\{(\phi(g), g) \mid g \in\left(\boldsymbol{A} \otimes \boldsymbol{A}^{o p}\right)^{n_{1}}\right\} .
$$

Sei $n_{Q}:=n_{1}+n_{2}$. Mit $\left(\boldsymbol{A} \otimes \boldsymbol{A}^{o p}\right)^{n_{1}} \oplus\left(\boldsymbol{A} \otimes \boldsymbol{A}^{o p}\right)^{n_{2}} \cong\left(\boldsymbol{A} \otimes \boldsymbol{A}^{o p}\right)^{n_{Q}}$ ist $\Gamma(\phi)$ Untermodul eines freien Moduls. Wir setzen:

$$
Q:=\left(\boldsymbol{A} \otimes \boldsymbol{A}^{o p}\right)^{n_{Q}} / \Gamma(\phi)
$$

und erhalten ähnlich wie im Abschnitt1.3.2 eine exakte Sequenz:

$$
0 \rightarrow \bar{Q} \hookrightarrow\left(\boldsymbol{A} \otimes \boldsymbol{A}^{o p}\right)^{n_{Q}} \rightarrow Q \rightarrow 0,
$$

wobei $\bar{Q}=\Gamma(\phi)$. Sei die Ordnung auf $\mathcal{F}_{\boldsymbol{n}_{\boldsymbol{A}}+n_{Q}}$ die Silbenordnung zu den Trennungsbuchstaben $\boldsymbol{f}_{1}<\ldots \boldsymbol{f}_{n_{1}}<\boldsymbol{e}_{1}<\cdots<\boldsymbol{e}_{n_{2}}$, wobei $\boldsymbol{f}_{1}, \ldots, \boldsymbol{f}_{n_{1}}$ die Erzeuger des Urbildmoduls und $\boldsymbol{e}_{1}, \ldots, \boldsymbol{e}_{n_{2}}$ die Erzeuger des Bildmoduls sind.

Wie in Abschnitt 1.3.2 konstruieren wir aus einem Erzeugersystem für $\bar{Q}$ ein Reduktionssystem $r_{Q}$.

\section{Bemerkung 1.3.20 (Wahl des Erzeugersystems)}

Von der Wahl des Erzeugersystems für $\bar{Q}$ hängt es ab, ob das induzierte $r_{Q}$ geeignet ist (vgl. 1.3.11). 


\section{Lemma 1.3.21 (Erzeugersystem)}

Seien $\boldsymbol{f}_{1}, \ldots, \boldsymbol{f}_{n_{1}}$ die Erzeuger des Urbildmoduls, dann ist

$$
\left\{\phi\left(\boldsymbol{f}_{i}\right), \boldsymbol{f}_{i} \mid i=1 \ldots n_{1}\right\}
$$

ein Erzeugersystem für $\Gamma(\phi)=\bar{Q}$.

Beweis: [Lemma 1.3.21] Sei $g \in\left(\boldsymbol{A} \otimes \boldsymbol{A}^{o p}\right)^{n_{1}}$, dann lässt es sich schreiben als:

$$
g=\sum_{\sigma=1}^{N} b_{\sigma} \boldsymbol{f}_{i_{\sigma}} c_{\sigma},
$$

wobei $b_{\sigma}, c_{\sigma} \in \boldsymbol{A} \otimes \boldsymbol{A}^{o p}$ und $i_{\sigma} \in\left\{1, \ldots, n_{1}\right\}$. Da $\phi$ ein $\boldsymbol{A} \otimes \boldsymbol{A}^{o p}$-Homomorphismus ist, gilt:

$$
\phi(g)=\sum_{\sigma=1}^{N} b_{\sigma} \phi\left(\boldsymbol{f}_{i_{\sigma}}\right) c_{\sigma} .
$$

Also lässt sich jedes $(\phi(g), g) \in \Gamma(\phi)$ durch $\left(\phi\left(\boldsymbol{f}_{i}\right), \boldsymbol{f}_{i}\right)$ erzeugen.

Die Reduktionsregeln in $r_{Q}$ lassen sich wegen Bemerkung 1.3.2 zerlegen in:

$$
\begin{aligned}
r_{f} & :=\left\{(x, y) \in r_{Q} \mid i \in\left\{1, \ldots, n_{1}\right\}, p, s \in \mathcal{M} \text { mit } x=p \boldsymbol{f}_{i} s\right\} \text { und } \\
r_{e} & :=\left\{(x, y) \in r_{Q} \mid i \in\left\{1, \ldots, n_{2}\right\}, p, s \in \mathcal{M} \text { mit } x=p \boldsymbol{e}_{i} s\right\} .
\end{aligned}
$$

Bemerkung 1.3.22 (Ordnung)

Nach der gewählten Ordnung ist jeder Erzeuger des Urbild-Moduls kleiner als ein Erzeuger des Bild-Moduls. Da eine Regel $(x, y) \in r_{f}$ verkleinert, enthält auch jeder Summand, der in $y$ vorkommt, jeweils genau einen Erzeuger des Urbild-Moduls.

\begin{tabular}{|c|c|c|c|c|}
\hline Name : & $g$ & $\rightarrow$ & $\bar{g}$ & mit \\
\hline$\widetilde{\operatorname{Rel}}:$ & $\lambda_{1} w+^{\prime} \lambda_{2} w+^{\prime} q$ & $\rightarrow$ & $\left(\lambda_{1}+\lambda_{2}\right) w+^{\prime} q$ & \\
\hline $\operatorname{Rel}_{A}$ & $\lambda w x \bar{w}+^{\prime} q$ & $\rightarrow$ & $\lambda w(y) \bar{w}+^{\prime} q$ & $(x, y) \in r_{\boldsymbol{A}}$ \\
\hline $\operatorname{Rel}_{f}:$ & $\lambda p x s+{ }^{\prime} q$ & $\rightarrow$ & $\lambda p(y) s+^{\prime} q$ & $(x, y) \in r_{f}$ \\
\hline $\operatorname{Rel}_{e}:$ & $\lambda p x s+^{\prime} q$ & $\rightarrow$ & $\lambda p(y) s{+^{\prime}}^{\prime} q$ & $(x, y) \in r_{e}$ \\
\hline
\end{tabular}

Sei im Folgenden $\widetilde{\mathcal{R}}_{\boldsymbol{A}, Q}$ das durch $r_{\boldsymbol{A}, Q}$ auf $\widetilde{\mathcal{F}}_{\boldsymbol{n}_{\boldsymbol{A}}+n_{Q}}$ induzierte Reduktionssystem, wobei $n_{Q}:=n_{1}+n_{2}$.

Bemerkung 1.3.23 (Rel)

Eine Reduktionsregel $(g, \bar{g}) \in \widetilde{\mathcal{R}}_{A, Q}$ ist von genau einer der folgenden Formen:

Dabei sei $\lambda, \bar{\lambda} \in \mathbb{K}^{*}$, sowie $p, s \in \mathcal{M}$ und $w, \bar{w} \in \mathcal{M}_{\boldsymbol{A}, Q}$ und $q \in \widetilde{\mathcal{F}}_{\boldsymbol{n}_{\boldsymbol{A}}+n_{Q}}$. 
Ähnlich wie $\varpi_{\boldsymbol{A}}$ im Abschnitt 1.3.2 nutzen wir im Folgenden oft die beiden Projektionen

$$
\begin{aligned}
& \pi_{0}: \tilde{\mathcal{F}}(0) \rightarrow \boldsymbol{A}, \\
& \pi_{1}: \widetilde{\mathcal{F}}(1) \rightarrow\left(\boldsymbol{A} \otimes \boldsymbol{A}^{o p}\right)^{n_{Q}},
\end{aligned}
$$

wobei $\widetilde{\mathcal{F}}(0)$ bzw. $\widetilde{\mathcal{F}}(1)$ die Teilmengen aus $\widetilde{\mathcal{F}}_{\boldsymbol{n}_{\boldsymbol{A}}+n_{Q}}$ bezeichnen, deren Elemente nur Summanden, mit keinem bzw. genau einem Erzeuger enthalten.

Die Menge der Elemente aus $\widetilde{\mathcal{F}}(1)$, deren Summanden genau einen Erzeuger aus dem Urbildmodul enthalten, bezeichnen wir mit $\widetilde{\mathcal{F}}\left(1_{f}\right)$.

Sei $\xi: \widetilde{\mathcal{F}}_{\boldsymbol{n}_{\boldsymbol{A}}+n_{Q}} \rightarrow \widetilde{\mathcal{F}}_{\boldsymbol{n}_{\boldsymbol{A}}+n_{Q}}$ der Homomorphismus, der dadurch definiert ist, dass er die Erzeuger des Urbildmoduls $\boldsymbol{f}_{i}$ auf Null abbildet und für alle anderen Erzeuger die Identität ist.

Bemerkung 1.3.24 (Kern $(\xi))$

Jeder Summand eines Elements aus $\widetilde{\mathcal{F}}\left(1_{f}\right)$ enthält einen Erzeuger des Urbildmoduls, also gilt $\widetilde{\mathcal{F}}\left(1_{f}\right) \subset \operatorname{Kern}(\xi)$.

Wir zerlegen $\widetilde{\mathcal{R}}_{\boldsymbol{A}, Q}$ in die Teilmengen:

$$
\begin{aligned}
& \mathcal{R}_{\xi}:=\left\{(g, \bar{g}) \in \widetilde{\mathcal{R}}_{\boldsymbol{A}, Q} \mid \xi(g)=\xi(\bar{g}) \text { und } x \in \widetilde{\mathcal{F}}(1)\right\} \\
& \mathcal{R}_{\Xi}:=\widetilde{\mathcal{R}}_{\boldsymbol{A}, Q}-\mathcal{R}_{\xi} .
\end{aligned}
$$

Wir wollen uns zunächst überlegen, wie die Regeln in $\mathcal{R}_{\xi}$ aussehen.

\section{Bemerkung 1.3.25 (Regeln in $\mathcal{R}_{\xi}$ )}

Sei $(g, \bar{g}) \in \mathcal{R}_{\xi}$, dann gilt mit den Bezeichnungen aus Bemerkung 1.3.23.

- Falls $(g, \bar{g}) \in \widetilde{\operatorname{Re} l}$, dann ist $w \in \widetilde{\mathcal{F}}\left(1_{f}\right)$.

- Falls $(g, \bar{g}) \in \operatorname{Rel}_{\boldsymbol{A}}$, dann ist $w x \bar{w} \in \widetilde{\mathcal{F}}\left(1_{f}\right)$.

- Die Regel $(g, \bar{g})$ liegt nicht in $\operatorname{Re}_{e}$.

- Falls $(g, \bar{g}) \in \operatorname{Rel}_{f}$, dann ist $p x s, p y s \in \widetilde{\mathcal{F}} 1_{f}$.

Es wird also niemals eine Regel aus $r_{e}$ genutzt, aber es kann jeweils vorkommen, dass $q$ einen Erzeuger $\boldsymbol{e}_{i}$ des Bildmoduls hat.

\section{Definition 1.3.26 (induzierter Modul $L$ )}

Sei $\varpi_{\boldsymbol{A}}: \widetilde{\mathcal{F}}(1) \rightarrow\left(\boldsymbol{A} \otimes \boldsymbol{A}^{o p}\right)^{n_{Q}}$ wie im Abschnitt 1.3.2 konstruiert. Sei $L$ der Untermodul des Urbildmoduls, der folgendermaßen definiert ist:

$$
L:=\left\langle\pi_{1}(x)-\pi_{1}(y) \mid(x, y) \in r_{f}\right\rangle .
$$

Wir nennen $L$ auch den durch $r_{f}$ induzierten Modul. 


\section{Lemma 1.3.27 (Modul L)}

Seien $g_{1}, g_{n} \in \widetilde{\mathcal{F}}(1)$ mit $g<\mathcal{R}_{\xi}>q_{n}$, dann gilt:

$$
\pi_{1}(g)-\pi_{1}\left(g_{n}\right) \in L
$$

Beweis: [Lemma 1.3.27] Sei $g_{1}<\mathcal{R}_{\xi}>q_{n}$, dann gibt es eine endliche Folge $\left(g_{i}, g_{i+1}\right)$, wobei $\left(g_{i}, g_{i+1}\right) \in \mathcal{R}_{\xi}$ oder $\left(g_{i+1}, g_{i}\right) \in \mathcal{R}_{\xi}$. Für die Behauptung reicht es also zu zeigen: Wenn $(g, \bar{g}) \in \mathcal{R}_{\xi}$, dann auch $\pi_{1}(g)-\pi_{1}(\bar{g}) \in L$.

Sei $(g, \bar{g}) \in \mathcal{R}_{\xi}$. Mit den Bezeichnern aus Bemerkung 1.3.23 unterscheiden wir folgende Fälle:

- Falls $(g, \bar{g}) \in \widetilde{\operatorname{Rel}}$, dann gilt:

$$
\begin{aligned}
\pi_{1}(g) & =\pi_{1}\left(\lambda_{1} w+{ }^{\prime} \lambda_{2} w+{ }^{\prime} q\right)=\lambda_{1} \pi_{1}(w)+\lambda_{2} \pi_{1}(w)+\pi_{1}(q) \\
& =\pi_{1}\left(\left(\lambda_{1}+\lambda_{2}\right) w\right)+\pi_{1}(q)=\pi_{1}\left(\left(\lambda_{1}+\lambda_{2}\right) w+{ }^{\prime} q\right) \\
& =\pi_{1}(\bar{g}) .
\end{aligned}
$$

Also gilt $\pi_{1}(g)-\pi_{1}(\bar{g})=0 \in L$.

- Falls $(g, \bar{g}) \in \operatorname{Rel}_{A}$, dann liegt entweder $w$ oder $\bar{w}$ in $\widetilde{\mathcal{F}}(1)$. Sei $\bar{w} \in \widetilde{\mathcal{F}}(1)$. Es ist $\pi_{0}(x)=\pi_{0}(y)$, da $(x, y) \in r_{\boldsymbol{A}}$, und daher gilt:

$$
\begin{aligned}
\pi_{1}(g) & =\pi_{1}\left(\lambda w x \bar{w}+{ }^{\prime} q\right)=\lambda \pi_{0}(w) \pi_{0}(x) \pi_{1}(\bar{w})+\pi_{1}(q) \\
& =\lambda \pi_{0}(w) \pi_{0}(y) \pi_{1}(\bar{w})+\pi_{1}(q)=\pi_{1}\left(\lambda w y \bar{w}+{ }^{\prime} q\right) \\
& =\pi_{1}(\bar{g}) .
\end{aligned}
$$

Also gilt $\pi_{1}(g)-\pi_{1}(\bar{g})=0 \in L$. Den Fall $w \in \widetilde{\mathcal{F}}(1)$ behandeln wir analog.

- Falls $(g, \bar{g}) \in \operatorname{Rel}_{f}$, dann gilt:

$$
\begin{aligned}
& \pi_{1}(g)=\pi_{1}\left(\lambda p x s+^{\prime} q\right)=\lambda \pi_{0}(p) \pi_{1}(x) \pi_{0}(s)+\pi_{1}(q), \\
& \pi_{1}(\bar{g})=\pi_{1}\left(\lambda p y s+^{\prime} q\right)=\lambda \pi_{0}(p) \pi_{1}(y) \pi_{0}(s)+\pi_{1}(q) .
\end{aligned}
$$

Also gilt wegen $\pi_{1}(x)-\pi_{1}(y) \in L$ auch $\pi_{1}(g)-\pi_{1}(\bar{g}) \in L$.

Sei im Folgenden $[\widetilde{\mathcal{F}}]:=\widetilde{\mathcal{F}}_{\boldsymbol{n}_{\boldsymbol{A}}+n_{Q}} / \underset{\mathcal{R}_{\xi}}{<\ldots .>}$ und sei

$$
\left[\widetilde{\mathcal{R}}_{\boldsymbol{A}, Q}\right]:=\left\{([g],[\bar{g}]) \in[\widetilde{\mathcal{F}}] \times[\widetilde{\mathcal{F}}] \mid(g, \bar{g}) \in \widetilde{\mathcal{R}}_{\boldsymbol{A}, Q} \text { und }[g] \neq[\bar{g}]\right\} .
$$

Lemma 1.3.28 (Äquivalenzklassen sind verträglich mit $\widetilde{\mathcal{F}}\left(1_{f}\right)$ )

Sei $g \in \widetilde{\mathcal{F}}\left(1_{f}\right)$, dann gilt das auch für jeden Repräsentanten in $[g]$.

Beweis: [Lemma 1.3.28] Sei $g \in \widetilde{\mathcal{F}}\left(1_{f}\right)$ und sei $g_{n} \in[g]$, dann ist $g=g_{1}<{ }_{\mathcal{R}_{\xi}} q_{n}$, also gibt es eine endliche Folge $\left(g_{i}, g_{i+1}\right)$, wobei $\left(g_{i}, g_{i+1}\right) \in \mathcal{R}_{\xi}$ oder $\left(g_{i+1}, g_{i}\right) \in \mathcal{R}_{\xi}$. Für die Behauptung reicht es also zu zeigen: Wenn $(g, \bar{g}) \in \mathcal{R}_{\xi}$ und $g$ oder $\bar{g} \in \mathcal{F}\left(1_{f}\right)$, dann liegen beide in $\widetilde{\mathcal{F}}\left(1_{f}\right)$.

Sei $(g, \bar{g}) \in \mathcal{R}_{\xi}$. Mit den Bezeichnern aus Bemerkung 1.3.23 unterscheiden wir folgende Fälle: 
- Falls $(g, \bar{g}) \in \widetilde{\mathrm{Rel}}$, dann führt die Regel nur zwei Terme mit gleichen Monomen zusammen; es kommen in $\bar{g}$ die gleichen Monome wie in $g$ vor.

Also gilt $g \in \widetilde{\mathcal{F}}\left(1_{f}\right) \Leftrightarrow \bar{g} \in \widetilde{\mathcal{F}}\left(1_{f}\right)$.

- Falls $(g, \bar{g}) \in \operatorname{Rel}_{\boldsymbol{A}}$, dann ist $x, y \in \widetilde{\mathcal{F}}(0)$. Da entweder gilt $g$ oder $\bar{g} \in \widetilde{\mathcal{F}}\left(1_{f}\right)$, muss $q \in \widetilde{\mathcal{F}}\left(1_{f}\right)$ und entweder $w$ oder $\bar{w} \in \widetilde{\mathcal{F}}\left(1_{f}\right)$ sein.

Also gilt $g \in \widetilde{\mathcal{F}}\left(1_{f}\right) \Leftrightarrow \bar{g} \in \widetilde{\mathcal{F}}\left(1_{f}\right)$.

- Falls $(g, \bar{g}) \in \operatorname{Rel}_{f}$, dann betrachten wir die folgenden zwei Fälle:

- Sei $g \in \widetilde{\mathcal{F}}\left(1_{f}\right)$, dann sind auch $p x s, q \in \widetilde{\mathcal{F}}\left(1_{f}\right)$. Mit Bemerkung 1.3.22 ist $y \in \widetilde{\mathcal{F}}\left(1_{f}\right)$ und da $p, s \in \mathcal{M}$, gilt auch pys $\in \widetilde{\mathcal{F}}\left(1_{f}\right)$.

- Sei $\bar{g} \in \widetilde{\mathcal{F}}\left(1_{f}\right)$, dann ist auch $q \in \widetilde{\mathcal{F}}\left(1_{f}\right)$. Da $(x, y) \in r_{f}$, ist $x \in \widetilde{\mathcal{F}}\left(1_{f}\right)$ und da $p, s \in \mathcal{M}$, gilt auch $p x s \in \widetilde{\mathcal{F}}\left(1_{f}\right)$.

Lemma 1.3.29 (Reduktionsregeln sind verträglich mit $\widetilde{\mathcal{F}}\left(1_{f}\right)$ )

$\operatorname{Sei}(g, \bar{g}) \in \widetilde{\mathcal{R}}_{\boldsymbol{A}, Q}$ mit $g \in \widetilde{\mathcal{F}}\left(1_{f}\right)$, dann gilt:

1. $(g, \bar{g}) \in \mathcal{R}_{\xi}$.

2. $\bar{g} \in \widetilde{\mathcal{F}}\left(1_{f}\right)$.

Beweis: [Lemma 1.3.29] $\mathrm{Zu} 1$.

Sei $(g, \bar{g}) \in \widetilde{\mathcal{R}}_{\boldsymbol{A}, Q}$ und $g \in \widetilde{\mathcal{F}}\left(1_{f}\right)$. Mit den Bezeichnern aus Bemerkung 1.3.23 unterscheiden wir folgende Fälle:

- Falls $(g, \bar{g}) \in \widetilde{\text { Rel}}$, dann führt die Regel nur zwei Terme mit gleichen Monomen zusammen; es kommen in $\bar{g}$ die gleichen Monome wie in $g$ vor.

Also folgt aus $g \in \widetilde{\mathcal{F}}\left(1_{f}\right)$ auch $\bar{g} \in \widetilde{\mathcal{F}}\left(1_{f}\right)$ und deshalb gilt $\xi(g)=0=\xi(\bar{g})$.

- Falls $(g, \bar{g}) \in \operatorname{Rel}_{A}$, dann ist $x, y \in \widetilde{\mathcal{F}}(0)$. Da entweder gilt $g$ oder $\bar{g} \in \widetilde{\mathcal{F}}\left(1_{f}\right)$, muss entweder $w$ oder $\bar{w} \in \widetilde{\mathcal{F}}\left(1_{f}\right)$ sein.

Also folgt $g \in \widetilde{\mathcal{F}}\left(1_{f}\right)$ und auch $\bar{g} \in \widetilde{\mathcal{F}}\left(1_{f}\right)$ und deshalb gilt $\xi(g)=0=\xi(\bar{g})$.

- Falls $(g, \bar{g}) \in \operatorname{Rel}_{f}$, dann folgt aus Bemerkung 1.3.22 dass auch $\bar{g} \in \widetilde{\mathcal{F}}\left(1_{f}\right)$

$\mathrm{Zu} 2$. :

Mit 1. folgt aus $(g, \bar{g}) \in \widetilde{\mathcal{R}}_{A, Q}$, so dass gilt: $[g]=[\bar{g}]$. Mit Lemma 1.3.28 folgt dann die Behauptung.

Satz 1.3.30 (Kern)

Sei $\left[\widetilde{\mathcal{R}}_{A, Q}\right]$ konvergent, dann gilt:

$$
\left\langle\pi_{1}(x-y) \mid(x, y) \in r_{f}\right\rangle=\operatorname{Kern}(\phi) .
$$

Beweis: [Satz 1.3.30

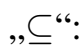


Sei $(x, y) \in r_{f}$, dann ist wegen Bemerkung $1.3 .22\left(0, \pi_{1}(x-y)\right) \in \bar{Q}=\Gamma(\phi)$. Also ist $\phi\left(\pi_{1}(x-y)\right)=0$.

,卫“":

Sei $h \in \operatorname{Kern}(\phi)$, dann ist $(0, h) \in \Gamma(\phi)$. Wegen Satz 1.3 .16 gilt $(0, h) \underset{\mathcal{R}_{\boldsymbol{A}, Q}}{\prec_{2}}(0,0)$.

Da wir jede Regel aus $\mathcal{R}$ auch als einen Weg in $\widetilde{\mathcal{R}}$ schreiben können (vgl. 1.2.16, gilt:

$$
\begin{aligned}
& (0, h) \underset{\mathcal{R}_{\boldsymbol{A}, Q}}{<\ldots \ldots .}(0,0) \text { und } \\
& {[(0, h)] \underset{\left[\widetilde{\mathcal{R}}_{\boldsymbol{A}, Q}\right]}{\stackrel{\cdots}{\cdots}]}[(0,0)] .}
\end{aligned}
$$

Nun ist $\left[\widetilde{\mathcal{R}}_{\boldsymbol{A}, Q}\right]$ konvergent, es gibt also Wege:

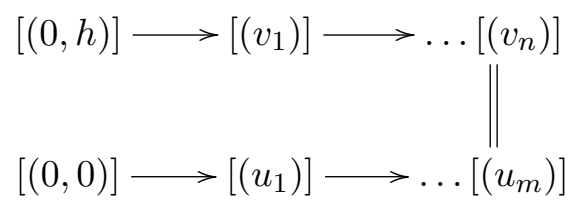

und wir erhalten folgendes Diagramm:

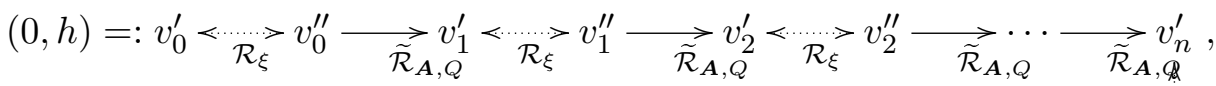

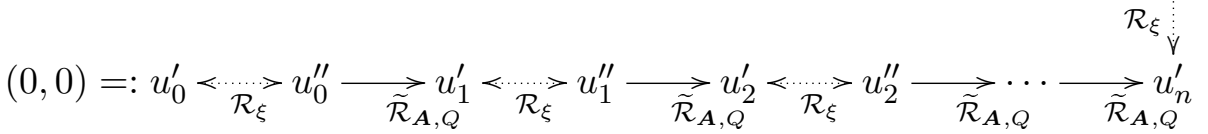

wobei $v_{i}^{\prime \prime}, v_{i}^{\prime} \in\left[v_{i}\right]$ und $u_{i}^{\prime \prime}, u_{i}^{\prime} \in\left[u_{i}\right]$.

Nach Lemma 1.3.29 und Lemma 1.3.28 liegen alle $v_{i}^{\prime \prime}, v_{i}^{\prime}, u_{i}^{\prime \prime}, u_{i}^{\prime} \in \mathcal{F}\left(1_{f}\right)$. Nach Lemma 1.3.29 gilt $\pi_{1}\left(v_{i}^{\prime}\right)=\pi_{1}\left(v_{i+1}^{\prime}\right)$ und $\pi_{1}\left(u_{i}^{\prime}\right)=\pi_{1}\left(u_{i+1}^{\prime}\right)$. Zusammen mit Lemma 1.3 .27 folgt:

$$
\pi_{1}\left(v_{i}^{\prime}\right)-\pi_{1}\left(v_{i}^{\prime \prime}\right), \pi_{1}\left(u_{i}^{\prime}\right)-\pi_{1}\left(u_{i}^{\prime \prime}\right) \in L .
$$

Um ein Erzeugersystem für den Kern zu erhalten, reicht es also ein konvergentes $\left[\mathcal{R}_{\boldsymbol{A}, Q}\right]$ zu kennen. Wenn $\mathcal{R}_{\boldsymbol{A}, Q}$ konvergent ist, dann ist es auch $\left[\mathcal{R}_{\boldsymbol{A}, Q}\right]$. Um die Konvergenz von $\mathcal{R}_{A, Q}$ nachzuweisen kann man zeigen, dass alle $\mathcal{P}$-minimalen Überschneidungen behebbar sind (vgl. 1.3.18). Wir wollen nun zeigen, dass wir nicht alle diese Überschneidungen überprüfen müssen.

Zunächst werden wir zeigen, dass die Regeln aus $\mathcal{R}_{\xi}$ mit denen aus $\mathcal{R}_{\Xi}$ kommutieren. Dazu betrachten wir folgendes Lemma.

Lemma 1.3.31 ( $\mathcal{R}_{\Xi}$ kommutiert mit $\left.\mathcal{R}_{\xi}\right)$

- Sei $\left(g_{1}, g_{2}\right) \in \mathcal{R}_{\xi}$ und $\left(g_{2}, h_{2}\right) \in \mathcal{R}_{\Xi}$, dann gibt es ein $h_{1} \in \widetilde{\mathcal{F}}(1)$, so dass $\left(g_{1}, h_{1}\right) \in \mathcal{R}_{\Xi}$ und $\left(h_{1}, h_{2}\right) \in \mathcal{R}_{\xi}$, also

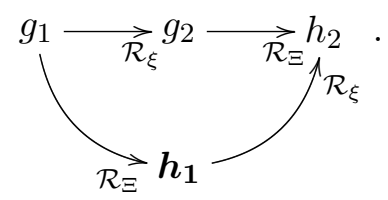


- Sei $\left(g_{1}, g_{2}\right) \in \mathcal{R}_{\xi}$ und $\left(g_{1}, h_{1}\right) \in \mathcal{R}_{\Xi}$, dann gibt es ein $h_{2} \in \widetilde{\mathcal{F}}(1)$, so dass $\left(h_{1}, h_{2}\right) \in \mathcal{R}_{\xi}$ und $\left(g_{2}, h_{2}\right) \in \mathcal{R}_{\Xi}$, also

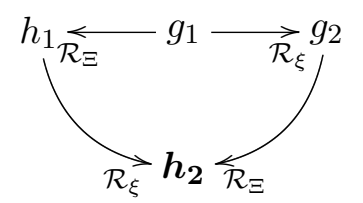

- Sei $\left(g_{1}, h_{1}\right) \in \mathcal{R}_{\Xi}$ und sei $g_{2} \underset{\mathcal{R}_{\xi}}{<\ldots} g_{1}$, dann gibt es ein $h_{2} \in \widetilde{\mathcal{F}}(1)$ mit $\left(g_{2}, h_{2}\right) \in \mathcal{R}_{\Xi}$ und $h_{2}<\ldots \ldots \mathcal{R}_{\xi}>h_{1}$, also

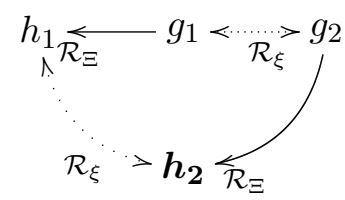

Beweis: [Lemma 1.3.31] Ein Element $g \in \widetilde{\mathcal{F}}(1)$ können wir zerlegen in einen Anteil in $g_{\xi} \in \widetilde{\mathcal{F}}\left(1_{f}\right)$ und einen Anteil $g_{\Xi}$, der nur Monome mit jeweils einem Erzeuger $\boldsymbol{e}_{i}$ des Bildmoduls enthält:

$$
g=\left(g_{\Xi}, g_{\xi}\right)
$$

Sei $\left(\left(g_{\Xi}, g_{\xi}\right) \rightarrow \bar{g}\right) \in \mathcal{R}_{\xi}$ eine Regel, die mit $g$ beginnt; sie verändert nur die zweite Stelle, also $\bar{g}=\left(g_{\Xi}, \bar{g}_{\xi}\right)$.

Da $\widetilde{\mathcal{R}}_{\boldsymbol{A}, Q}$ durch $r_{\boldsymbol{A}, \boldsymbol{M}}$ induziert ist, gibt es für jedes $v \in \widetilde{\mathcal{F}}(1)$ die Regel $\left(\left(v, g_{\xi}\right) \rightarrow\right.$ $\left.\left(v, \bar{g}_{\xi},\right)\right) \in \mathcal{R}_{\xi}$.

Sei $\left(\left(g_{\Xi}, g_{\xi}\right) \rightarrow h\right) \in \mathcal{R}_{\Xi}$ eine Regel, die mit $g$ beginnt; sie verändert $g_{\xi}$ nicht, also ist $h=\left(h_{\Xi}, h_{\xi}+^{\prime} g_{\xi}\right)$.

Da $\widetilde{\mathcal{R}}_{\boldsymbol{A}, Q}$ durch $r_{\boldsymbol{A}, \boldsymbol{M}}$ induziert ist, gibt es für jedes $v \in \widetilde{\mathcal{F}}(1)$ die Regel $\left(\left(g_{\Xi}, v\right) \rightarrow\right.$ $\left.\left(h_{\Xi}, h_{\xi}+{ }^{\prime} v\right)\right) \in \mathcal{R}_{\Xi}$.

Also „kommutiert“ jede Regel aus $\mathcal{R}_{\Xi}$ mit jeder aus $\mathcal{R}_{\xi}$, weshalb die beiden ersten Punkte gelten. Den letzten Punkt zeigt man durch mehrfaches Anwenden der ersten beiden.

\section{Definition 1.3.32 (schwach vollständig)}

Sei $r_{\boldsymbol{A}, Q}:=r_{\boldsymbol{A}} \cup r_{Q}$ und sei $\mathcal{P}_{Q}$ das Modulprädikat. Falls jede $\mathcal{P}_{Q}$-minimale Überschneidung $(w, \pi, \bar{\pi})$ mit $\pi, \bar{\pi} \in r_{\boldsymbol{A}} \cup r_{e}$ mit Regeln aus $\widetilde{\mathcal{R}}_{\boldsymbol{A}, Q}$ behebbar ist, dann nennen wir $r_{A, Q}$ schwach vollständig.

Satz 1.3.33 (Hauptsatz)

Sei $r_{A, Q}$ schwach vollständig, dann ist

$$
\left[\widetilde{\mathcal{R}}_{\boldsymbol{A}, Q}\right] \subset \widetilde{\mathcal{F}}(1) \times \widetilde{\mathcal{F}}(1) \text { lokal konfluent. }
$$

Beweis: [Satz 1.3.33] Sei $r_{\boldsymbol{A}, Q}$ schwach vollständig und seien $([g],[h]),([g],[\bar{h}])$ zwei 
Reduzierungsregeln, die mit $[g]$ beginnen, dann existieren $g_{1}, g_{2} \in[g]$ und $h_{1} \in[h]$ sowie $\bar{h}_{2} \in[\bar{h}]$ mit:

$$
h<\mathcal{R}_{\xi}>h_{1} \overbrace{\widetilde{\mathcal{R}}_{\boldsymbol{A}, Q}}^{g_{1<\ldots \ldots}>g_{2}} \underbrace{}_{\widetilde{\mathcal{R}}_{\boldsymbol{A}, Q}} \bar{h}_{2}<\ldots \ldots>\bar{h} .
$$

Nun gilt $[g] \in \widetilde{\mathcal{F}}(1)$ und damit auch $g_{1}, g_{2}, h_{1}, h, \bar{h}_{1}, \bar{h} \in \widetilde{\mathcal{F}}(1)$. Da $\left(\left[g_{i}\right],\left[h_{i}\right]\right) \in$ $\left[\widetilde{\mathcal{R}}_{\boldsymbol{A}, Q}\right]$, ist $\left[g_{i}\right] \neq\left[h_{i}\right]$, also liegen $\left(g_{i}, h_{i}\right)$ in $\mathcal{R}_{\Xi}$. Mit Lemma 1.3.31 vereinfacht sich das Diagramm zu:

$$
h<_{\mathcal{R}_{\xi}}>h_{1}<_{\mathcal{R}_{\xi}}>\boldsymbol{h}_{\mathbf{2}} \swarrow_{\mathcal{R}_{\Xi}}^{g_{2}} \overbrace{\mathcal{R}_{\Xi}}{ }_{\bar{h}_{2}<\mathcal{R}_{\xi}}>\bar{h} .
$$

Da $\left(g_{2}, h_{2}\right),\left(g_{2}, \bar{h}_{2}\right) \in \mathcal{R}_{\Xi}$, sind sie von einer Regel aus $r_{\boldsymbol{A}} \cup r_{e}$ induziert, nach Voraussetzung und ähnlich wie Satz 1.3 .10 können wir zeigen, dass es Wege $h_{2} \ldots \ldots \ldots . .>z$ und $\bar{h} \quad \cdots . . . . . .>z$ gibt. Diese Wege sind auf den Äquivalenzklassen von folgender Form:

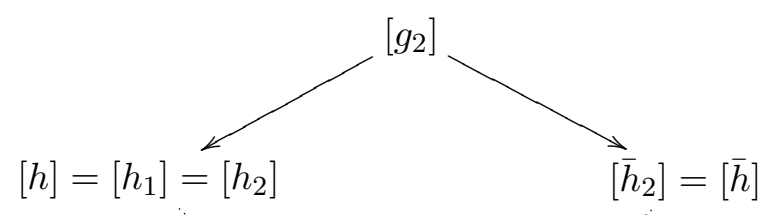

$[z]$.

Wir erhalten also ein Erzeugersystem für den Kern, indem wir nachweisen, dass alle minimalen Überschneidungen in $r_{\boldsymbol{A}, \boldsymbol{M}}$, die höchstens einen Erzeuger des Bildmoduls enthalten, bezüglich $\mathcal{R}_{A, Q}$ behebbar sind. 
Kapitel 2

\title{
Konvergentes Reduktionssystem
}

\author{
für $\mathcal{A}(n)$
}


In diesem Kapitel werden wir für die orthogonale freie Quantengruppe ein vollständiges Reduktionssystem angeben.

\subsection{Definition $\mathcal{A}(n)$}

Definition 2.1.1 $(\mathcal{A}(n))$

Sei $\mathcal{A}(n)$ die $\mathbb{K}$-Algebra, die von $a_{i, j}$, wobei $i, j \in\{1, \ldots, n\}$, und den Relationen

$$
\begin{aligned}
& \sum_{p=1}^{n} a_{i, p} a_{j, p}=\delta_{i, j} \\
& \sum_{p=1}^{n} a_{p, i} a_{p, j}=\delta_{i, j}
\end{aligned}
$$

erzeugt wird. Es gilt also $\mathcal{A}(n)=\mathcal{F}_{n^{2}} / I_{\mathcal{A}(n)}$, wobei

$$
I_{\mathcal{A}(n)}:=\left\langle\sum_{p=1}^{n} a_{i, p} a_{j, p}-\delta_{i, j}, \sum_{p=1}^{n} a_{p, i} a_{p, j}=\delta_{i, j} \mid i, j=1 \ldots n\right\rangle .
$$

Wir nennen $\mathcal{A}(n)$ die orthogonale freie Quantengruppe mit $n^{2}$-Erzeuger.

\section{Bemerkung 2.1.2}

Schreibt man die Erzeuger als Matrix:

$$
A:=\left(a_{i, j}\right)_{i, j},
$$

dann lassen sich die Relationen schreiben als:

$$
\begin{aligned}
& A A^{t}=\mathrm{id}, \\
& A^{t} A=\mathrm{id},
\end{aligned}
$$

wobei id die Identitätsmatrix und $A^{t}$ die transponierte Matrix von $A$ ist.

Satz 2.1.3 (vollständiges Reduktionssystem $r_{\mathcal{A}(n)}$ )

$$
\begin{aligned}
\text { Sei } r_{\mathcal{A}(n)} & :=\left\{\widetilde{Z}_{p, q}, \widetilde{S}_{p, q}, \widetilde{Z}_{p, q, r}, \widetilde{S}_{p, q, r} \mid p, q, r=1, \ldots, n\right\}, \text { wobei } \\
\widetilde{Z}_{p, q} & :=\left(a_{p, 1} a_{q, 1},-\sum_{i=2}^{n} a_{p, i} a_{q, i}+\delta_{p, q}\right), \\
\widetilde{S}_{p, q} & :=\left(a_{1, p} a_{1, q},-\sum_{i=2}^{n} a_{i, p} a_{i, q}+\delta_{p, q}\right), \\
\widetilde{Z}_{p, q, r} & :=\left(a_{p, 1} a_{q, 2} a_{r, 2},-a_{p, 1} \sum_{i=3}^{n} a_{q, i} a_{r, i}+a_{p, 1} \delta_{q, r}-\left(-\sum_{i=2}^{n} a_{p, i} a_{q, i}+\delta_{p, q}\right) a_{r, 1}\right), \\
\widetilde{S}_{p, q, r} & :=\left(a_{1, p} a_{2, q} a_{2, r},-a_{1, p} \sum_{i=3}^{n} a_{i, q} a_{i, r}+a_{1, p} \delta_{q, r}-\left(-\sum_{i=2}^{n} a_{i, p} a_{i, q}+\delta_{p, q}\right) a_{1, r}\right),
\end{aligned}
$$

dann ist $r_{\mathcal{A}(n)}$ mit der kanonischen Ordnung ein vollständiges Wortersetzungssystem für $\mathcal{A}(n)$.

Bemerkung 2.1.4 (Spezialfälle $n=1$ und $n=2$ )

Falls $n=1$, gibt es nur einen Erzeuger $a_{1,1}$ und nur eine Relation $\left(a_{1,1}^{2}, 1\right)$.

Falls $n=2$, fällt in $\widetilde{Z}_{p, q, r}$ und $\widetilde{S}_{p, q, r}$ auf der rechten Seite jeweils die erste Summe weg. 


\section{Vereinbarung 2.1.5}

Um die Lesbarkeit zu erhöhen, setzen wir:

$$
\begin{aligned}
Z_{p, q} & :=-\sum_{i=2}^{n} a_{p, i} a_{q, i}+\delta_{p, q}, \\
S_{p, q} & :=-\sum_{i=2}^{n} a_{i, p} a_{i, q}+\delta_{p, q}, \\
Z_{p, q, r} & :=-a_{p, 1} \sum_{i=3}^{n} a_{q, i} a_{r, i}+a_{p, 1} \delta_{q, r}, \\
S_{p, q, r} & :=-a_{1, p} \sum_{i=3}^{n} a_{i, q} a_{i, r}+a_{1, p} \delta_{q, r} .
\end{aligned}
$$

Die Regeln in $\tilde{r}_{A(n)}$ verkürzen sich dann zu:

$$
\begin{aligned}
a_{p, 1} a_{q, 1} & \mid \stackrel{\widetilde{Z}_{p, q}}{\longrightarrow} Z_{p, q}, \\
a_{1, p} a_{1, q} & \mid \stackrel{\widetilde{S}_{p, q}}{\longrightarrow} S_{p, q}, \\
a_{p, 1} a_{q, 2} a_{r, 2} & \stackrel{\widetilde{Z}_{p, q, r}}{\longrightarrow} Z_{p, q, r}-Z_{p, q} a_{r, 1}, \\
a_{1, p} a_{2, q} a_{2, r} & \stackrel{\widetilde{S}_{p, q, r}}{\longrightarrow} S_{p, q, r}-S_{p, q} a_{1, r} .
\end{aligned}
$$

Bevor wir den Satz 2.1.3 beweisen, bemerken wir noch, dass wegen Satz 1.2.46 und der Definition 1.2.40, das durch $r_{\mathcal{A}(n)}$ induzierte Reduktionssystem konvergent ist.

Beweis: [Satz 2.1.3] Es sind zwei Dinge zu zeigen:

1. Es gilt: $\left\langle x-y \mid(x, y) \in r_{\mathcal{A}(n)}\right\rangle=I_{\mathcal{A}(n)}$.

2. Das Ersetzungssystem $r_{\mathcal{A}(n)}$ ist vollständig.

$\mathrm{Zu} 1$.:

Es gilt: Nach Definition ist

$$
I_{\mathcal{A}(n)}:=\left\langle-\left(\widetilde{Z}_{p, q}-a_{p, 1} a_{q, 1}\right),-\left(\widetilde{S}_{p, q}-a_{p, 1} a_{q, 1}\right) \mid p, q=1, \ldots, n\right\rangle .
$$

Also gilt: $I_{\mathcal{A}(n)} \subset \tilde{r}_{\mathcal{A}(n)}$.

Andererseits sehen wir, dass $Z_{p, q, r}, S_{p, q, r} \subset I_{\mathcal{A}(n)}$ gilt durch folgende Gleichungen:

$$
\begin{aligned}
Z_{p, q} a_{r_{1}} & =a_{p, 1} a_{q, 1} a_{r_{1}}=a_{p, 1} Z_{q, r}, \\
S_{p, q} a_{1, r} & =a_{1, p} a_{1, q} a_{1, r}=a_{1, p} S_{q, r} .
\end{aligned}
$$

$\mathrm{Zu} 2$. :

Um Vollständigkeit zu zeigen müssen alle minimalen Überschneidungen behebbar sein. In der folgenden Tabelle sind alle minimalen Überschneidungen aufgeführt. Ein Eintrag in der Tabelle besteht aus drei Teilen: der Länge der Überschneidung, dem Monom, für das es zwei Reduzierungsregeln (Zeile $\cdot s$ und $p \cdot$ Spalte) gibt, und einer 
Seitenangabe, auf der die Überschneidung behoben wird.

\begin{tabular}{|c|c|c|}
\hline & $\widetilde{Z}_{x, y}=a_{x, 1} a_{y, 1}$ & $\widetilde{S}_{x, y}=a_{1, x} a_{1, y}$ \\
\hline$\widetilde{Z}_{p, q}$ & $\begin{array}{l}1: a_{p, 1} a_{q, 1} a_{y, 1} \\
2: \text { beide gleich }\end{array}$ & $\begin{array}{l}1: a_{p, 1} a_{1,1} a_{1, y} \\
2: a_{1,1} a_{1,1}\end{array}$ \\
\hline$\widetilde{S}_{p, q}$ & $\begin{array}{ll}1: a_{1, p} a_{1,1} a_{y, 1} & 2.2 .5 \\
2: a_{1,1} a_{1,1} & 2.2 .6\end{array}$ & $\begin{array}{l}1: a_{1, p} a_{1, q} a_{1, y} \\
2: \text { beide gleich }\end{array}$ \\
\hline$\widetilde{Z}_{p, q, r}$ & $\begin{array}{l}1: \text { nicht möglich } \\
2: \text { nicht möglich }\end{array}$ & $\begin{array}{l}1: a_{p, 1} a_{q, 2} a_{1,2} a_{1, y} \\
2: a_{p, 1} a_{1,2} a_{1,2}\end{array}$ \\
\hline$\widetilde{S}_{p, q, r}$ & $\begin{array}{ll}1: a_{1, p} a_{2, q} a_{2,1} a_{y, 1} & 2.2 .14 \\
2: a_{1, p} a_{2,1} a_{2,1} & 2.2 .16\end{array}$ & $\begin{array}{l}1: \text { nicht möglich } \\
2: \text { nicht möglich }\end{array}$ \\
\hline & $\widetilde{Z}_{x, y, z}=a_{x, 1} a_{y, 2} a_{z, 2}$ & $\widetilde{S}_{x, y, z}=a_{1, x} a_{2, y} a_{2, z}$ \\
\hline$\widetilde{Z}_{p, q}$ & $\begin{array}{l}1: a_{p, 1} a_{q, 1} a_{y, 2} a_{z, 2} \\
2: \text { nicht möglich }\end{array}$ & $\begin{array}{ll}1: a_{p, 1} a_{1,1} a_{2, y} a_{2, z} & 2.2 .9 \\
2: a_{1,1} a_{2,1} a_{2, z} & 2.2 .11\end{array}$ \\
\hline$\widetilde{S}_{p, q}$ & $\begin{array}{ll}1: a_{1, p} a_{1,1} a_{y, 2} a_{z, 2} & 2.2 .10 \\
2: a_{1,1} a_{1,2} a_{z, 2} & \end{array}$ & $\begin{array}{l}1: a_{1, p} a_{1,1} a_{2, y} a_{2, z} \\
2: \text { nicht möglich }\end{array}$ \\
\hline$\widetilde{Z}_{p, q, r}$ & $\begin{array}{l}1: \text { nicht möglich } \\
2: \text { nicht möglich } \\
3: \text { beide gleich }\end{array}$ & \begin{tabular}{ll|}
$1: a_{p, 1} a_{q, 2} a_{1,2} a_{2, y} a_{2, z}$ & 2.2 .17 \\
$2: a_{p, 1} a_{1,2} a_{2,2} a_{2, z}$ & 2.2 .19 \\
$3: a_{1,1} a_{2,2} a_{2,2}$ & 2.2 .21 \\
\end{tabular} \\
\hline$\widetilde{S}_{p, q, r}$ & $\begin{array}{ll}1: a_{1, p} a_{2, q} a_{2,1} a_{y, 2} a_{z, 2} & 2.2 .18 \\
2: a_{1, p} a_{2,1} a_{2,2} a_{z, 2} & \\
3: a_{1,1} a_{2,2} a_{2,2} & \end{array}$ & $\begin{array}{l}1: \text { nicht möglich } \\
2: \text { nicht möglich } \\
3: \text { beide gleich }\end{array}$ \\
\hline
\end{tabular}

\subsection{Behebung der minimalen Überschneidungen}

In den folgenden Rechnungen steht $\delta_{x, y}$ immer für das Kroneckersymbol,

$$
\delta_{x, y}=\left\{\begin{array}{cc}
1 & \text { für } x=y \\
0 & \text { sonst }
\end{array} .\right.
$$




\section{Bemerkung 2.2.1 (Symmetrie)}

Durch Vertauschen der Indizes aus $Z_{p, q}$ erhält man $S_{p, q}$, und durch Vertauschen der Indizes aus $Z_{p, q, r}$ erhält man $S_{p, q, r}$, weshalb einige der Rechnungen symmetrisch sind.

\subsubsection{Rechenregeln}

\section{Satz 2.2.2 (Rechenregel)}

In der freien Algebra $\mathcal{F}_{n^{2}}$ gilt:

1 .

$$
\sum_{j=2}^{n} Z_{p, j} a_{j, y}=\sum_{i=2}^{n} a_{p, i} S_{i, y}+\left(\delta_{1, y}-\delta_{p, 1}\right) a_{p, y}
$$

2.

$$
\sum_{j=3}^{n} Z_{p, 1, j} a_{j, z}=\sum_{i=3}^{n} a_{p, 1} S_{i, i, z}-\left(1-\delta_{1, z}-\delta_{2, z}\right) a_{p, 1} a_{1, z}
$$

3.

$$
\sum_{j=2}^{n} Z_{p, j, j}=\sum_{i=3}^{n} a_{p, 1} S_{i, i}+a_{p, 1}
$$

4.

$$
\sum_{j=3}^{n} Z_{p, 1} a_{j, 1} a_{j, z}=\sum_{i=2}^{n} a_{p, i} S_{i, 1, z}-\delta_{1, z} \sum_{i=2}^{n} a_{p, i} a_{1, i}+\delta_{p, 1} \sum_{j=3}^{n} a_{j, 1} a_{j, z}
$$

5.

$$
Z_{p, q, 1}\left(\sum_{j=3}^{n} a_{j, y} a_{j, z}-\delta_{y, z}\right)=a_{p, 1} \sum_{i=3}^{n} a_{q, i} S_{i, y, z}+\delta_{q, 1} a_{p, 1}\left(\sum_{j=3}^{n} a_{j, y} a_{j, z}-\delta_{y, z}\right)
$$

6.

$$
\sum_{j=2}^{n} Z_{p, q, j} a_{j, y}=\sum_{i=3}^{n} a_{p, 1} a_{q, i} S_{i, y}+\delta_{y, 1} a_{p, 1} a_{q, 1}+\delta_{y, 2} a_{p, 1} a_{q, 2}-\delta_{q, 1} a_{p, 1} a_{1, y}
$$

Beweis: [Satz 2.2.2] 
Zu 1.:

$$
\begin{aligned}
& \sum_{j=2}^{n} Z_{p, j} a_{j, y} \\
& =-\sum_{j=2}^{n}\left(\sum_{i=2}^{n} a_{p, i} a_{j, i}-\delta_{p, j}\right) a_{j, y} \\
& =-\sum_{i=2}^{n} \sum_{j=2}^{n} a_{p, i} a_{j, i} a_{j, y}+(\sum_{i=2}^{n} a_{p, i} \delta_{i, y}-\underbrace{\sum_{i=2}^{n} a_{p, i} \delta_{i, y}}_{=a_{p, y}\left(1-\delta_{1, y}\right)})+\underbrace{\sum_{j=2}^{n} \delta_{p, j} a_{j, y}}_{=\left(1-\delta_{p, 1}\right) a_{p, y}} \\
& =\sum_{i=2}^{n} a_{p, i} S_{i, y}+\left(\delta_{1, y}-\delta_{p, 1}\right) a_{p, y}
\end{aligned}
$$

$\mathrm{Zu} 2$. :

$$
\begin{aligned}
& \sum_{j=3}^{n} Z_{p, 1, j} a_{j, z} \\
= & -\sum_{j=3}^{n}\left(a_{p, 1} \sum_{i=3}^{n} a_{1, i} a_{j, i}-a_{p, 1} \delta_{1, j}\right) a_{j, z} \\
= & -\sum_{i=3}^{n} \sum_{j=3}^{n} a_{p, 1} a_{1, i} a_{j, i} a_{j, z} \\
= & -\sum_{i=3}^{n} a_{p, 1}\left(a_{1, i} \sum_{j=3}^{n} a_{j, i} a_{j, z}-a_{1, i} \delta_{i, z}+a_{1, i} \delta_{i, z}\right) \\
= & \sum_{i=3}^{n} a_{p, 1} S_{i, i, z}-\sum_{i=3}^{n} a_{p, 1} a_{1, i} \delta_{i, z} \\
= & \sum_{i=3}^{n} a_{p, 1} S_{i, i, z}-\left(1-\delta_{1, z}-\delta_{2, z}\right) a_{p, 1} a_{1, z}
\end{aligned}
$$

$\mathrm{Zu} 3$. :

$$
\begin{aligned}
& \sum_{j=2}^{n} Z_{p, j, j} \\
= & -\sum_{j=2}^{n}\left(a_{p, 1} \sum_{i=3}^{n} a_{j, i} a_{j, i}-a_{p, 1} \delta_{j, j}\right) \\
= & -\sum_{i=3}^{n} a_{p, 1}\left(\sum_{j=2}^{n} a_{j, i} a_{j, i}-\delta_{i, i}\right)+a_{p, 1} \\
= & \sum_{i=3}^{n} a_{p, 1} S_{i, i}+a_{p, 1}
\end{aligned}
$$


$\mathrm{Zu}$ 4.:

$$
\begin{aligned}
& \sum_{j=3}^{n} Z_{p, 1} a_{j, 1} a_{j, z} \\
= & -\sum_{j=3}^{n}\left(\sum_{i=2}^{n} a_{p, i} a_{1, i}-\delta_{p, 1}\right) a_{j, 1} a_{j, z} \\
= & -\sum_{i=2}^{n} \sum_{j=3}^{n} a_{p, i} a_{1, i} a_{j, 1} a_{j, z}+\sum_{j=3}^{n} \delta_{p, 1} a_{j, 1} a_{j, z} \\
= & -\sum_{i=2}^{n} a_{p, i}\left(a_{1, i} \sum_{j=3}^{n} a_{j, 1} a_{j, z}-a_{1, i} \delta_{1, z}+a_{1, i} \delta_{1, z}\right)+\sum_{j=3}^{n} \delta_{p, 1} a_{j, 1} a_{j, z} \\
= & \sum_{i=2}^{n} a_{p, i} S_{i, 1, z}-\sum_{i=2}^{n} a_{p, i} a_{1, i} \delta_{1, z}+\sum_{j=3}^{n} \delta_{p, 1} a_{j, 1} a_{j, z}
\end{aligned}
$$

$\mathrm{Zu}$ 5.:

$$
\begin{aligned}
& Z_{p, q, 1}\left(\sum_{j=3}^{n} a_{j, y} a_{j, z}-\delta_{y, z}\right) \\
= & \left(-a_{p, 1} \sum_{i=3}^{n} a_{q, i} a_{1, i}+a_{p, 1} \delta_{q, 1}\right)\left(\sum_{j=3}^{n} a_{j, y} a_{j, z}-\delta_{y, z}\right) \\
= & -a_{p, 1} \sum_{i=3}^{n} a_{q, i}\left(a_{1, i} \sum_{j=3}^{n} a_{j, y} a_{j, z}-a_{1, i} \delta_{y, z}\right)+\delta_{q, 1} a_{p, 1}\left(\sum_{j=3}^{n} a_{j, y} a_{j, z}-\delta_{y, z}\right) \\
= & a_{p, 1} \sum_{i=3}^{n} a_{q, i} S_{i, y, z}+\delta_{q, 1} a_{p, 1}\left(\sum_{j=3}^{n} a_{j, y} a_{j, z}-\delta_{y, z}\right)
\end{aligned}
$$


$\mathrm{Zu} 6 .:$

$$
\begin{aligned}
& \sum_{j=2}^{n} Z_{p, q, j} a_{j, y} \\
= & -\sum_{j=2}^{n}\left(a_{p, 1} \sum_{i=3}^{n} a_{q, i} a_{j, i}-a_{p, 1} \delta_{q, j}\right) a_{j, y} \\
= & -\sum_{i=3}^{n} a_{p, 1} a_{q, i}\left(\sum_{j=2}^{n} a_{j, i} a_{j, y}-\delta_{i, y}+\delta_{i, y}\right)+\sum_{j=2}^{n} a_{p, 1} \delta_{q, j} a_{j, y} \\
= & -\sum_{i=3}^{n} a_{p, 1} a_{q, i}\left(\sum_{j=2}^{n} a_{j, i} a_{j, y}-\delta_{i, y}\right)-\sum_{i=3}^{n} a_{p, 1} a_{q, i} \delta_{i, y}+\sum_{j=2}^{n} a_{p, 1} \delta_{q, j} a_{j, y} \\
= & \sum_{i=3}^{n} a_{p, 1} a_{q, i} S_{i, y}-\left(1-\delta_{y, 1}-\delta_{y, 2}\right) a_{p, 1} a_{q, y}+\left(1-\delta_{q, 1}\right) a_{p, 1} a_{q, y} \\
= & \sum_{i=3}^{n} a_{p, 1} a_{q, i} S_{i, y}+\delta_{y, 1} a_{p, 1} a_{q, 1}+\delta_{y, 2} a_{p, 1} a_{q, 2}-\delta_{q, 1} a_{p, 1} a_{1, y}
\end{aligned}
$$




\subsubsection{Reduktionswege für $a_{p, 1} a_{q, 1} a_{y, 1}$}

Beginnend mit $\left(a_{p, 1} a_{q, 1} a_{y, 1}, Z_{p, q} a_{y, 1}\right)$ :

$$
\underline{a_{p, 1} a_{q, 1} a_{y, 1}} \stackrel{\widetilde{Z}_{p, q}}{\longrightarrow} Z_{p, q} a_{y, 1}
$$

Beginnend mit $\left(a_{p, 1} a_{q, 1} a_{y, 1}, a_{p, 1} Z_{q, y}\right)$ :

$$
\begin{aligned}
& a_{p, 1} \underline{a_{q, 1} a_{y, 1}} \mid \stackrel{\widetilde{Z}_{q, y}}{\longrightarrow} a_{p, 1} Z_{q, y}=a_{p, 1}\left(-\sum_{i=2}^{n} a_{q, i} a_{y, i}+\delta_{q, y}\right) \\
& =-\underline{a_{p, 1} a_{q, 2} a_{y, 2}}+Z_{p, q, y} \\
& \stackrel{\widetilde{Z}_{p, q, y}}{\longrightarrow}-Z_{p, q, y}+Z_{p, q} a_{y, 1}+Z_{p, q, y} \\
& =Z_{p, q} a_{y, 1}
\end{aligned}
$$

Bemerkung 2.2.3 (Symmetrie)

Diese Rechnungen sind nach Vertauschen der Indizes identisch mit denen in 2.2.3. 
2.2.3 Reduktionswege für $a_{1, p} a_{1, q} a_{1, y}$

Beginnend mit $\left(a_{1, p} a_{1, q} a_{1, y}, S_{p, q} a_{1, y}\right)$ :

$$
\underline{a_{1, p} a_{1, q}} a_{1, y} \stackrel{\widetilde{S}_{p, q}}{\longrightarrow} S_{p, q} a_{1, y}
$$

Beginnend mit $\left(a_{1, p} a_{1, q} a_{1, y}, a_{1, p} S_{q, y}\right)$ :

$$
\begin{aligned}
a_{1, p} a_{1, q} a_{1, y} & \stackrel{\widetilde{S}_{q, y}}{\longrightarrow} a_{1, p} S_{q, y}=a_{1, p}\left(-\sum_{i=2}^{n} a_{i, q} a_{i, y}+\delta_{q, y}\right) \\
& =-\underline{a_{1, p} a_{2, q} a_{2, y}}+S_{p, q, y} \\
1 \stackrel{\widetilde{S}_{p, q, y}}{\longrightarrow} & -S_{p, q, y}+S_{p, q} a_{1, y}+S_{p, q, y} \\
& =S_{p, q} a_{1, y}
\end{aligned}
$$

\section{Bemerkung 2.2.4 (Symmetrie)}

Diese Rechnungen sind nach Vertauschen der Indizes identisch mit denen in 2.2.2. 


\subsubsection{Reduktionswege für $a_{p, 1} a_{1,1} a_{1, y}$}

Beginnend mit $\left(a_{p, 1} a_{1,1} a_{1, y}, Z_{p, 1} a_{1, y}\right)$ :

$$
\begin{aligned}
& \underline{a_{p, 1} a_{1,1} a_{1, y}} \mid \stackrel{\widetilde{Z}_{p, 1}}{\longrightarrow}\left(-\sum_{i=2}^{n} a_{p, i} a_{1, i}+\delta_{p, 1}\right) a_{1, y} \\
& =-\sum_{i=2}^{n} a_{p, i} \underline{a_{1, i} a_{1, y}}+\delta_{p, 1} a_{1, y} \\
& \stackrel{\widetilde{S}_{i, y}}{\longrightarrow}-\sum_{i=2}^{n} a_{p, i} S_{i, y}+\delta_{p, 1} a_{1, y} \\
& =\sum_{i=2}^{n} a_{p, i}\left(\sum_{j=2}^{n} a_{j, i} a_{j, y}-\delta_{i, y}\right)+\delta_{p, 1} a_{1, y} \\
& =\sum_{i=2}^{n} \sum_{j=2}^{n} a_{p, i} a_{j, i} a_{j, y}-\left(1-\delta_{1, y}\right) a_{p, y}+\delta_{p, 1} a_{1, y} \\
& =\sum_{i=2}^{n} \sum_{j=2}^{n} a_{p, i} a_{j, i} a_{j, y}-a_{p, y}+\delta_{1, y} a_{p, 1}+\delta_{p, 1} a_{1, y}
\end{aligned}
$$

Beginnend mit $\left(a_{p, 1} a_{1,1} a_{1, y}, a_{p, 1} S_{1, y}\right)$ :

$$
\begin{aligned}
a_{p, 1} a_{1,1} a_{1, y} \mid \stackrel{\widetilde{S}_{1, y}}{\longrightarrow} & -a_{p, 1}\left(\sum_{j=2}^{n} a_{j, 1} a_{j, y}-\delta_{1, y}\right) \\
= & -\sum_{j=2}^{n} \frac{a_{p, 1} a_{j, 1} a_{j, y}+a_{p, 1} \delta_{1, y}}{\widetilde{Z}_{p, j}} \longrightarrow \\
\longrightarrow & -\sum_{j=2}^{n} Z_{p, j} a_{j, y}+a_{p, 1} \delta_{1, y} \\
= & \sum_{j=2}^{n}\left(\sum_{i=2}^{n} a_{p, i} a_{j, i}-\delta_{p, j}\right) a_{j, y}+a_{p, 1} \delta_{1, y} \\
= & \sum_{j=2}^{n} \sum_{i=2}^{n} a_{p, i} a_{j, i} a_{j, y}-\left(1-\delta_{p, 1}\right) a_{p, y}+a_{p, 1} \delta_{1, y} \\
= & \sum_{j=2}^{n} \sum_{i=2}^{n} a_{p, i} a_{j, i} a_{j, y}-a_{p, y}+\delta_{p, 1} a_{1, y}+\delta_{1, y} a_{p, 1}
\end{aligned}
$$

Bemerkung 2.2.5 (Symmetrie)

Diese Rechnungen sind nach Vertauschen der Indizes identisch mit denen in 2.2.5. 


\subsubsection{Reduktionswege für $a_{1, p} a_{1,1} a_{y, 1}$}

Beginnend mit $\left(a_{1, p} a_{1,1} a_{y, 1}, S_{p, 1} a_{y, 1}\right)$ :

$$
\begin{aligned}
a_{1, p} a_{1,1} a_{y, 1} \mid \stackrel{\widetilde{S}_{p, 1}}{\longrightarrow} & S_{p, 1} a_{y, 1}=\left(-\sum_{i=2}^{n} a_{i, p} a_{i, 1}+\delta_{p, 1}\right) a_{y, 1} \\
& =-\sum_{i=2}^{n} a_{i, p} \underline{a_{i, 1} a_{y, 1}}+\delta_{1, p} a_{y, 1} \\
\mid \stackrel{\widetilde{Z}_{i, y}}{\longrightarrow} & -\sum_{i=2}^{n} a_{i, p} Z_{i, y}+\delta_{1, p} a_{y, 1} \\
& =\sum_{i=2}^{n} a_{i, p}\left(\sum_{j=2}^{n} a_{i, j} a_{y, j}-\delta_{i, y}\right)+\delta_{1, p} a_{y, 1} \\
& =\sum_{i=2}^{n} \sum_{j=2}^{n} a_{i, p} a_{i, j} a_{y, j}-\underbrace{\sum_{i=2}^{n} a_{i, p} \delta_{i, y}}_{=\left(1-\delta_{y, 1}\right) a_{1, p}}+\delta_{1, p} a_{y, 1}
\end{aligned}
$$

Beginnend mit $\left(a_{1, p} a_{1,1} a_{y, 1}, a_{1, p} Z_{1, y}\right)$ :

$$
\begin{aligned}
a_{1, p} \underline{a_{1,1} a_{y, 1}} \mid \stackrel{\widetilde{Z}_{1, y}}{\longrightarrow} a_{1, p} Z_{1, y}=-a_{1, p}\left(\sum_{j=2}^{n} a_{1, j} a_{y, j}-\delta_{1, y}\right) \\
=-\sum_{j=2}^{n} \frac{a_{1, p} a_{j, 1}}{a_{i, y}}+a_{1, p} \delta_{y, 1} \\
\mid \stackrel{\widetilde{S}_{p, j}}{\longrightarrow}-\sum_{j=2}^{n} S_{p, j} a_{y, j}+a_{1, p} \delta_{y, 1} \\
=\sum_{j=2}^{n}\left(\sum_{i=2}^{n} a_{i, p} a_{i, j}-\delta_{p, j}\right) a_{y, j}+a_{1, p} \delta_{y, 1} \\
=\sum_{j=2}^{n} \sum_{i=2}^{n} a_{i, p} a_{i, j} a_{y, j}-\underbrace{\sum_{j=2}^{n} \delta_{p, j} a_{y, j}}_{=\left(1-\delta_{p, 1}\right) a_{y, 1}}+a_{1, p} \delta_{y, 1}
\end{aligned}
$$

Bemerkung 2.2.6 (Symmetrie)

Diese Rechnungen sind nach Vertauschen der Indizes identisch mit denen in 2.2.4. 


\subsubsection{Reduktionswege für $a_{1,1} a_{1,1}$}

Beginnend mit $\left(a_{1,1} a_{1,1}, Z_{1,1}\right)$ :

$$
\begin{aligned}
& a_{1,1} a_{1,1} \mid \stackrel{\widetilde{Z}_{1,1}}{\longrightarrow}-\sum_{i=2}^{n} a_{1, i} a_{1, i}+\delta_{1,1} \\
& \mid \stackrel{\widetilde{S}_{i, i}}{\longrightarrow} \sum_{i=2}^{n}\left(\sum_{j=2}^{n} a_{j, i} a_{j, i}-\delta_{i, i}\right)+1
\end{aligned}
$$

Beginnend mit $\left(a_{1,1} a_{1,1}, S_{1,1}\right)$ :

$$
\begin{aligned}
\stackrel{a_{1,1} a_{1,1}}{ } \mid \stackrel{\widetilde{S}_{1,1}}{\longrightarrow}-\sum_{j=2}^{n} a_{j, 1} a_{j, 1}+\delta_{1,1} \\
\mid \stackrel{\widetilde{Z}_{j, j}}{\longrightarrow} \sum_{j=2}^{n}\left(\sum_{i=2}^{n} a_{j, i} a_{j, i}-\delta_{j, j}\right)+1
\end{aligned}
$$


2.2.7 Reduktionswege für $a_{p, 1} a_{q, 1} a_{y, 2} a_{z, 2}$

Beginnend mit $\left(a_{p, 1} a_{q, 1} a_{y, 2} a_{z, 2}, Z_{p, q} a_{y, 2} a_{z, 2}\right)$ :

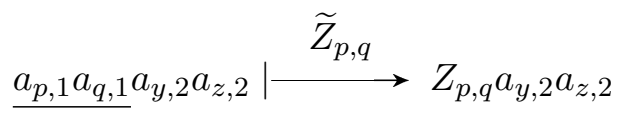

Beginnend mit $\left(a_{p, 1} a_{q, 1} a_{y, 2} a_{z, 2}, a_{p, 1}\left(Z_{q, y, z}-Z_{q, y} a_{z, 1}\right)\right)$ :

$$
\begin{aligned}
& a_{p, 1} \underline{a_{q, 1} a_{y, 2} a_{z, 2}} \mid \stackrel{\widetilde{Z}_{q, y, z}}{\longrightarrow} a_{p, 1}\left(Z_{q, y, z}-Z_{q, y} a_{z, 1}\right) \\
& =-a_{p, 1}\left(a_{q, 1} \sum_{i=3}^{n} a_{y, i} a_{z, i}-a_{q, 1} \delta_{y, z}\right) \\
& +a_{p, 1}\left(\sum_{i=2}^{n} a_{q, i} a_{y, i}-\delta_{q, y}\right) a_{z, 1} \\
& =-\underline{a_{p, 1} a_{q, 1}}\left(\sum_{i=3}^{n} a_{y, i} a_{z, i}-\delta_{y, z}\right) \\
& +\left(\underline{a_{p, 1} a_{q, 2} a_{y, 2}}-Z_{p, q, y}\right) a_{z, 1} \\
& \mid \stackrel{\widetilde{Z}_{p, q}, \widetilde{Z}_{p, q, y}}{\longrightarrow}-Z_{p, q}\left(\sum_{i=3}^{n} a_{y, i} a_{z, i}-\delta_{y, z}\right) \\
& +\left(Z_{p, q, y}-Z_{p, q} a_{y, 1}-Z_{p, q, y}\right) a_{z, 1} \\
& =-Z_{p, q}\left(\sum_{i=3}^{n} a_{y, i} a_{z, i}-\delta_{y, z}+\underline{a_{y, 1} a_{z, 1}}\right) \\
& \mid \stackrel{\widetilde{Z}_{y, z}}{\longrightarrow}=-Z_{p, q}\left(\sum_{i=3}^{n} a_{y, i} a_{z, i}-\delta_{y, z}+Z_{y, z}\right) \\
& =-Z_{p, q}\left(-a_{2, y} a_{2, z}\right)
\end{aligned}
$$

\section{Bemerkung 2.2.7 (Symmetrie)}

Diese Rechnungen sind nach Vertauschen der Indizes identisch mit denen in 2.2.8. 


\subsubsection{Reduktionswege für $a_{1, p} a_{1, q} a_{2, y} a_{2, z}$}

Beginnend mit $\left(a_{1, p} a_{1, q} a_{2, y} a_{2, z}, S_{p, q} a_{2, y} a_{2, z}\right)$ :

$$
\underline{a_{1, p} a_{1, q}} a_{2, y} a_{2, z} \mid \stackrel{\widetilde{S}_{p, q}}{\longrightarrow} S_{p, q} a_{2, y} a_{2, z}
$$

Beginnend mit $\left(a_{1, p} a_{1, q} a_{2, y} a_{2, z}, a_{1, p}\left(S_{q, y, z}-S_{q, y} a_{1, z}\right)\right)$ :

$$
\begin{aligned}
& a_{1, p} \underline{a_{1, q} a_{2, y} a_{2, z}} \mid \stackrel{\widetilde{S}_{q, y, z}}{\longrightarrow} a_{1, p}\left(S_{q, y, z}-S_{q, y} a_{1, z}\right) \\
& =-a_{1, p}\left(a_{1, q} \sum_{i=3}^{n} a_{i, y} a_{i, z}-a_{1, q} \delta_{y, z}\right) \\
& +a_{1, p}\left(\sum_{i=2}^{n} a_{i, q} a_{i, y}-\delta_{q, y}\right) a_{1, z} \\
& =-\underline{a_{1, p} a_{1, q}}\left(\sum_{i=3}^{n} a_{i, y} a_{i, z}-\delta_{y, z}\right) \\
& +\left(\underline{a_{1, p} a_{q, 2} a_{2, y}}-S_{p, q, y}\right) a_{1, z} \\
& \stackrel{\widetilde{S}_{p, q}, \widetilde{S}_{p, q, y}}{\longrightarrow}-S_{p, q}\left(\sum_{i=3}^{n} a_{i, y} a_{i, z}-\delta_{y, z}\right) \\
& +\left(S_{p, q, y}-S_{p, q} a_{y, 1}-S_{p, q, y}\right) a_{1, z} \\
& =-S_{p, q}\left(\sum_{i=3}^{n} a_{i, y} a_{i, z}-\delta_{y, z}+\underline{a_{y, 1} a_{1, z}}\right) \\
& \mid \stackrel{\widetilde{S}_{y, z}}{\longrightarrow}=-S_{p, q}\left(\sum_{i=3}^{n} a_{i, y} a_{i, z}-\delta_{y, z}+S_{y, z}\right) \\
& =-S_{p, q}\left(-a_{2, y} a_{2, z}\right)
\end{aligned}
$$

Bemerkung 2.2.8 (Symmetrie)

Diese Rechnungen sind nach Vertauschen der Indizes identisch mit denen in 2.2.7. 
2.2.9 Reduktionswege für $a_{p, 1} a_{1,1} a_{2, y} a_{2, z}$

Bemerkung 2.2.9 (Symmetrie)

Diese Rechnungen sind nach Vertauschen der Indizes identisch mit denen in 2.2.10.

Beginnend mit $\left(a_{p, 1} a_{1,1} a_{2, y} a_{2, z}, Z_{p, 1} a_{2, y} a_{2, z}\right)$ :

$$
\begin{aligned}
& \underline{a_{p, 1} a_{1,1}} a_{2, y} a_{2, z} \mid \stackrel{\widetilde{Z}_{p, 1}}{\longrightarrow}\left(-\sum_{i=2}^{n} a_{p, i} a_{1, i}+\delta_{p, 1}\right) a_{2, y} a_{2, z} \\
& =-\sum_{i=2}^{n} a_{p, i} \underline{a_{1, i} a_{2, y} a_{2, z}}+\delta_{p, 1} a_{2, y} a_{2, z} \\
& \mid \stackrel{\widetilde{S}_{i, y, z}}{\longrightarrow}-\sum_{i=2}^{n} a_{p, i}\left(S_{i, y, z}-S_{i, y} a_{1, z}\right)+\delta_{p, 1} a_{2, y} a_{2, z} \\
& =\sum_{i=2}^{n} a_{p, i}\left(a_{1, i} \sum_{j=3}^{n} a_{j, y} a_{j, z}-a_{1, i} \delta_{y, z}\right) \\
& +\sum_{i=2}^{n} a_{p, i} S_{i, y} a_{1, z}+\delta_{p, 1} a_{2, y} a_{2, z} \\
& =\sum_{i=2}^{n} \sum_{j=3}^{n} a_{p, i} a_{1, i} a_{j, y} a_{j, z}-\sum_{i=2}^{n} a_{p, i} a_{1, i} \delta_{y, z} \\
& +\sum_{i=2}^{n} a_{p, i} S_{i, y} a_{1, z}+\delta_{p, 1} a_{2, y} a_{2, z} \\
& =\sum_{i=2}^{n} \sum_{j=3}^{n} a_{p, i} a_{1, i} a_{j, y} a_{j, z}+\left(Z_{p, 1}-\delta_{p, 1}\right) \delta_{y, z} \\
& +\sum_{i=2}^{n} a_{p, i} S_{i, y} a_{1, z}+\delta_{p, 1} a_{2, y} a_{2, z} \\
& =\sum_{i=2}^{n} \sum_{j=3}^{n} a_{p, i} a_{1, i} a_{j, y} a_{j, z}+Z_{p, 1} \delta_{y, z} \\
& +\sum_{i=2}^{n} a_{p, i} S_{i, y} a_{1, z}-\delta_{p, 1}\left(\delta_{y, z}-a_{2, y} a_{2, z}\right)
\end{aligned}
$$


Beginnend mit $\left(a_{p, 1} a_{1,1} a_{2, y} a_{2, z}, a_{p, 1}\left(S_{1, y, z}-S_{1, y} a_{1, z}\right)\right)$ :

$$
\begin{aligned}
& a_{p, 1} \underline{a_{1,1} a_{2, y} a_{2, z}} \\
& \stackrel{\widetilde{S}_{1, y, z}}{\longrightarrow}-a_{p, 1}\left(a_{1,1} \sum_{j=3}^{n} a_{j, y} a_{j, z}-a_{1,1} \delta_{y, z}\right) \\
& +a_{p, 1}\left(\sum_{j=2}^{n} a_{j, 1} a_{j, y}-\delta_{1, y}\right) a_{1, z} \\
& =-\underline{a_{p, 1} a_{1,1}}\left(\sum_{j=3}^{n} a_{j, y} a_{j, z}-\delta_{y, z}\right) \\
& +\sum_{j=2}^{n} \underline{a_{p, 1} a_{j, 1}} a_{j, y} a_{1, z}-a_{p, 1} \delta_{1, y} a_{1, z} \\
& \stackrel{\widetilde{Z}_{p, 1}, \widetilde{Z}_{p, j}}{\longrightarrow}\left(\sum_{i=2}^{n} a_{p, i} a_{1, i}-\delta_{p, 1}\right)\left(\sum_{j=3}^{n} a_{j, y} a_{j, z}-\delta_{y, z}\right) \\
& +\sum_{j=2}^{n} Z_{p, j} a_{j, y} a_{1, z}-a_{p, 1} \delta_{1, y} a_{1, z} \\
& \stackrel{2.2 .2}{=} \sum_{i=2}^{n} \sum_{j=3}^{n} a_{p, i} a_{1, i} a_{j, y} a_{j, z}-\delta_{p, 1} \sum_{j=3}^{n} a_{j, y} a_{j, z}+Z_{p, 1} \delta_{y, z} \\
& +\left(\sum_{i=2}^{n} a_{p, i} S_{i, y}+\left(\delta_{1, y}-\delta_{p, 1}\right) a_{p, y}\right) a_{1, z}-a_{p, 1} \delta_{1, y} a_{1, z} \\
& =\sum_{i=2}^{n} \sum_{j=3}^{n} a_{p, i} a_{1, i} a_{j, y} a_{j, z}+Z_{p, 1} \delta_{y, z}+\sum_{i=2}^{n} a_{p, i} S_{i, y} a_{1, z} \\
& -\delta_{p, 1}\left(\sum_{j=3}^{n} a_{j, y} a_{j, z}+\underline{a_{1, y} a_{1, z}}\right) \\
& \stackrel{\widetilde{S}_{y, z}}{\longrightarrow} \sum_{i=2}^{n} \sum_{j=3}^{n} a_{p, i} a_{1, i} a_{j, y} a_{j, z}+Z_{p, 1} \delta_{y, z}+\sum_{i=2}^{n} a_{p, i} S_{i, y} a_{1, z} \\
& -\delta_{p, 1}\left(\sum_{j=3}^{n} a_{j, y} a_{j, z}-\left(\sum_{j=2}^{n} a_{j, y} a_{j, z}-\delta_{y, z}\right)\right) \\
& =\sum_{i=2}^{n} \sum_{j=3}^{n} a_{p, i} a_{1, i} a_{j, y} a_{j, z}+Z_{p, 1} \delta_{y, z}+\sum_{i=2}^{n} a_{p, i} S_{i, y} a_{1, z} \\
& -\delta_{p, 1}\left(\delta_{y, z}-a_{2, y} a_{2, z}\right)
\end{aligned}
$$


2.2.10 Reduktionswege für $a_{1, p} a_{1,1} a_{y, 2} a_{z, 2}$

Bemerkung 2.2.10 (Symmetrie)

Diese Rechnungen sind nach Vertauschen der Indizes identisch mit denen in 2.2.9.

Beginnend mit $\left(a_{1, p} a_{1,1} a_{y, 2} a_{z, 2}, S_{p, 1} a_{y, 2} a_{z, 2}\right)$ :

$$
\begin{aligned}
& \underline{a_{1, p} a_{1,1}} a_{y, 2} a_{z, 2} \mid \stackrel{\widetilde{S}_{p, 1}}{\longrightarrow}\left(-\sum_{i=2}^{n} a_{i, p} a_{i, 1}+\delta_{p, 1}\right) a_{y, 2} a_{z, 2} \\
& =-\sum_{i=2}^{n} a_{i, p} \underline{a_{i, 1} a_{y, 2} a_{z, 2}}+\delta_{p, 1} a_{y, 2} a_{z, 2} \\
& \mid \stackrel{\widetilde{Z}_{i, y, z}}{\longrightarrow}-\sum_{i=2}^{n} a_{i, p}\left(Z_{i, y, z}-Z_{i, y} a_{z, 1}\right)+\delta_{p, 1} a_{y, 2} a_{z, 2} \\
& =\sum_{i=2}^{n} a_{i, p}\left(a_{i, 1} \sum_{j=3}^{n} a_{y, j} a_{z, j}-a_{i, 1} \delta_{y, z}\right) \\
& +\sum_{i=2}^{n} a_{i, p} Z_{i, y} a_{z, 1}+\delta_{p, 1} a_{y, 2} a_{z, 2} \\
& =\sum_{i=2}^{n} \sum_{j=3}^{n} a_{i, p} a_{i, 1} a_{y, j} a_{z, j}-\sum_{i=2}^{n} a_{i, p} a_{i, 1} \delta_{y, z} \\
& +\sum_{i=2}^{n} a_{i, p} Z_{i, y} a_{z, 1}+\delta_{p, 1} a_{y, 2} a_{z, 2} \\
& =\sum_{i=2}^{n} \sum_{j=3}^{n} a_{i, p} a_{i, 1} a_{y, j} a_{z, j}+\left(S_{p, 1}-\delta_{p, 1}\right) \delta_{y, z} \\
& +\sum_{i=2}^{n} a_{i, p} Z_{i, y} a_{z, 1}+\delta_{p, 1} a_{y, 2} a_{z, 2} \\
& =\sum_{i=2}^{n} \sum_{j=3}^{n} a_{i, p} a_{i, 1} a_{y, j} a_{z, j}+S_{p, 1} \delta_{y, z} \\
& +\sum_{i=2}^{n} a_{i, p} Z_{i, y} a_{z, 1}-\delta_{p, 1}\left(\delta_{y, z}-a_{y, 2} a_{z, 2}\right)
\end{aligned}
$$


Beginnend mit $\left(a_{1, p} a_{1,1} a_{y, 2} a_{z, 2}, a_{1, p}\left(Z_{1, y, z}-Z_{1, y} a_{z, 1}\right)\right)$ :

$$
\begin{aligned}
& a_{1, p} \underline{a_{1,1} a_{y, 2} a_{z, 2}} \\
& \stackrel{\widetilde{Z}_{1, y, z}}{\longrightarrow}-a_{1, p}\left(a_{1,1} \sum_{j=3}^{n} a_{y, j} a_{z, j}-a_{1,1} \delta_{y, z}\right) \\
& +a_{1, p}\left(\sum_{j=2}^{n} a_{1, j} a_{y, j}-\delta_{1, y}\right) a_{z, 1} \\
& =-\underline{a_{1, p} a_{1,1}}\left(\sum_{j=3}^{n} a_{y, j} a_{z, j}-\delta_{y, z}\right) \\
& +\sum_{j=2}^{n} \underline{a_{1, p} a_{1, j}} a_{y, j} a_{z, 1}-a_{1, p} \delta_{1, y} a_{z, 1} \\
& \mid \stackrel{\widetilde{S}_{p, 1}, \widetilde{S}_{p, j}}{\longrightarrow}\left(\sum_{i=2}^{n} a_{i, p} a_{i, 1}-\delta_{p, 1}\right)\left(\sum_{j=3}^{n} a_{y, j} a_{z, j}-\delta_{y, z}\right) \\
& +\sum_{j=2}^{n} S_{p, j} a_{y, j} a_{z, 1}-a_{1, p} \delta_{1, y} a_{z, 1} \\
& \stackrel{2.2 .2}{=} \sum_{i=2}^{n} \sum_{j=3}^{n} a_{i, p} a_{i, 1} a_{y, j} a_{z, j}-\delta_{p, 1} \sum_{j=3}^{n} a_{y, j} a_{z, j}+S_{p, 1} \delta_{y, z} \\
& +\left(\sum_{i=2}^{n} a_{i, p} Z_{i, y}+\left(\delta_{1, y}-\delta_{p, 1}\right) a_{y, p}\right) a_{z, 1}-a_{1, p} \delta_{1, y} a_{z, 1} \\
& =\sum_{i=2}^{n} \sum_{j=3}^{n} a_{i, p} a_{i, 1} a_{y, j} a_{z, j}+S_{p, 1} \delta_{y, z}+\sum_{i=2}^{n} a_{i, p} Z_{i, y} a_{z, 1} \\
& -\delta_{p, 1}\left(\sum_{j=3}^{n} a_{y, j} a_{z, j}+\underline{a_{y, 1} a_{z, 1}}\right) \\
& \stackrel{\widetilde{Z}_{y, z}}{\longrightarrow} \sum_{i=2}^{n} \sum_{j=3}^{n} a_{i, p} a_{i, 1} a_{y, j} a_{z, j}+S_{p, 1} \delta_{y, z}+\sum_{i=2}^{n} a_{i, p} Z_{i, y} a_{z, 1} \\
& -\delta_{p, 1}\left(\sum_{j=3}^{n} a_{y, j} a_{z, j}-\left(\sum_{j=2}^{n} a_{y, j} a_{z, j}-\delta_{y, z}\right)\right) \\
& =\sum_{i=2}^{n} \sum_{j=3}^{n} a_{i, p} a_{i, 1} a_{y, j} a_{z, j}+S_{p, 1} \delta_{y, z}+\sum_{i=2}^{n} a_{i, p} Z_{i, y} a_{z, 1} \\
& -\delta_{p, 1}\left(\delta_{y, z}-a_{y, 2} a_{z, 2}\right)
\end{aligned}
$$


2.2.11 Reduktionswege für $a_{1,1} a_{2,1} a_{2, z}$

Beginnend mit $\left(a_{1,1} a_{2,1} a_{2, z}, Z_{1,2} a_{2, z}\right)$ :

$$
\begin{aligned}
\underline{a_{1,1} a_{2,1}} a_{2, z} \mid \stackrel{\widetilde{Z}_{1,2}}{\longrightarrow} & =\left(-\sum_{i=2}^{n} a_{1, i} a_{2, i}+\delta_{1,2}\right) a_{2, z} \\
& =-\sum_{i=2}^{n} \underline{a_{1, i} a_{2, i} a_{2, z}} \\
\mid \stackrel{\widetilde{S}_{i, i, z}}{\longrightarrow} & -\sum_{i=2}^{n}\left(S_{i, i, z}-S_{i, i} a_{1, z}\right)
\end{aligned}
$$

Beginnend mit $\left(a_{1,1} a_{2,1} a_{1, z}, S_{1,1, z}-S_{1,1} a_{1, z}\right)$ :

$$
\begin{aligned}
& \underline{a_{1,1} a_{2,1} a_{1, z}} \\
& \mid \stackrel{\widetilde{S}_{1,1, z}}{\longrightarrow}\left(-a_{1,1} \sum_{i=3}^{n} a_{i, 1} a_{i, z}+a_{1,1} \delta_{1, z}\right)+\left(\sum_{j=2}^{n} \underline{a_{j, 1} a_{j, 1}}-\delta_{1,1}\right) a_{1, z} \\
& \stackrel{\widetilde{Z}_{j, j}}{\longrightarrow}-\sum_{j=3}^{n} \underline{a_{1,1} a_{j, 1}} a_{j, z}+a_{1,1} \delta_{1, z} \\
& -\sum_{i=2}^{n}\left(\sum_{j=2}^{n} a_{j, i} a_{j, i}-\delta_{j, j}\right) a_{1, z}-a_{1, z} \\
& \stackrel{\widetilde{Z}_{1, j}}{\longrightarrow} \sum_{j=3}^{n}\left(\sum_{i=2}^{n} a_{1, i} a_{j, i}-\delta_{1, j}\right) a_{j, z}+a_{1,1} \delta_{1, z} \\
& -\sum_{i=2}^{n}\left(\sum_{j=2}^{n} a_{j, i} a_{j, i}-\delta_{i, i}\right) a_{1, z}-a_{1, z} \\
& =\sum_{i=2}^{n} \sum_{j=3}^{n} a_{1, i} a_{j, i} a_{j, z}+a_{1,1} \delta_{1, z} \\
& +\sum_{i=2}^{n} S_{i, i} a_{1, z}-\left(a_{1,1} \delta_{1, z}+\sum_{i=2}^{n} a_{1, i} \delta_{i, z}\right) \\
& =-\sum_{i=2}^{n}\left(S_{i, i, z}-S_{i, i} a_{1, z}\right)
\end{aligned}
$$

Bemerkung 2.2.11 (Symmetrie)

Diese Rechnungen sind nach Vertauschen der Indizes identisch mit denen in 2.2.12) . 


\subsubsection{Reduktionswege für $a_{1,1} a_{1,2} a_{z, 2}$}

Beginnend mit $\left(a_{1,1} a_{1,2} a_{z, 2}, S_{1,2} a_{z, 2}\right)$ :

$$
\begin{aligned}
& \underline{a_{1,1} a_{1,2}} a_{z, 2} \mid \stackrel{\widetilde{S}_{1,2}}{\longrightarrow}=\left(-\sum_{i=2}^{n} a_{i, 1} a_{i, 2}+\delta_{1,2}\right) a_{z, 2} \\
& =-\sum_{i=2}^{n} \underline{a_{i, 1} a_{i, 2} a_{z, 2}} \\
& \stackrel{\widetilde{Z}_{i, i, z}}{\longrightarrow}-\sum_{i=2}^{n}\left(Z_{i, i, z}-Z_{i, i} a_{z, 1}\right)
\end{aligned}
$$

Beginnend mit $\left(a_{1,1} a_{1,2} a_{z, 1}, Z_{1,1, z}-Z_{1,1} a_{z, 1}\right)$ :

$$
\begin{aligned}
\mid \stackrel{\widetilde{Z}_{1,1, z}}{\longrightarrow} & \left(-a_{1,1} \sum_{i=3}^{n} a_{1, i} a_{z, i}+a_{1,1} \delta_{1, z}\right)+\left(\sum_{j=2}^{n} \frac{a_{1, j} a_{1, j}}{a_{z, 1}}-\delta_{1,1}\right) a_{z, 1} \\
\stackrel{\widetilde{S}_{j, j}}{\longrightarrow}- & -\sum_{j=3}^{n} \frac{a_{1,1} a_{1, j}}{{ }^{\prime}} a_{z, j}+a_{1,1} \delta_{1, z} \\
& -\sum_{i=2}^{n}\left(\sum_{j=2}^{n} a_{i, j} a_{i, j}-\delta_{j, j}\right) a_{z, 1}-a_{z, 1} \\
\widetilde{S}_{1, j} \rightarrow & \sum_{j=3}^{n}\left(\sum_{i=2}^{n} a_{i, 1} a_{i, j}-\delta_{1, j}\right) a_{z, j}+a_{1,1} \delta_{1, z} \\
& -\sum_{i=2}^{n}\left(\sum_{j=2}^{n} a_{i, j} a_{i, j}-\delta_{i, i}\right) a_{z, 1}-a_{z, 1} \\
= & \sum_{i=2}^{n} \sum_{j=3}^{n} a_{i, 1} a_{i, j} a_{z, j}+a_{1,1} \delta_{1, z} \\
& +\sum_{i=2}^{n} Z_{i, i} a_{z, 1}-\left(a_{1,1} \delta_{1, z}+\sum_{i=2}^{n} a_{i, 1} \delta_{i, z}\right) \\
= & -\sum_{i=2}^{n}\left(Z_{i, i, z}-Z_{i, i} a_{z, 1}\right)
\end{aligned}
$$

\section{Bemerkung 2.2.12 (Symmetrie)}

Diese Rechnungen sind nach Vertauschen der Indizes identisch mit denen in 2.2.11). 
2.2.13 Reduktionswege für $a_{p, 1} a_{q, 2} a_{1,2} a_{1, y}$

Beginnend mit $\left(a_{p, 1} a_{q, 2} a_{1,2} a_{1, y},\left(Z_{p, q, 1}-Z_{p, q} a_{1,1}\right) a_{1, y}\right)$ :

$$
\begin{aligned}
\mid \widetilde{Z}_{p, q, 1} & \frac{a_{p, 1} a_{q, 2} a_{1,2}}{\longrightarrow} a_{1, y} \\
\hline \widetilde{S}_{1, y} & \left(-a_{p, 1} \sum_{i=3}^{n} a_{q, i} a_{1, i}+a_{p, 1} \delta_{q, 1}\right) a_{1, y}-Z_{p, q} \underline{a_{1,1} a_{1, y}} \\
\hline \widetilde{S}_{i, y} & -a_{p, 1} \sum_{i=3}^{n} a_{q, i} \underline{a_{1, i} a_{1, y}}+a_{p, 1} \delta_{q, 1} a_{1, y}-Z_{p, q} S_{1, y} \\
\longrightarrow & \sum_{i=3}^{n} a_{p, 1} a_{q, i}\left(\sum_{j=2}^{n} a_{j, i} a_{j, y}-\delta_{i, y}\right)+a_{p, 1} \delta_{q, 1} a_{1, y}-Z_{p, q} S_{1, y} \\
= & \sum_{i=3}^{n} \sum_{i=2}^{n} a_{p, 1} a_{q, i} a_{j, i} a_{j, y}-Z_{p, q} S_{1, y} \\
& -\sum_{i=3}^{n} a_{p, 1} a_{q, i} \delta_{i, y}+a_{p, 1} \delta_{q, 1} a_{1, y} \\
= & \sum_{i=3}^{n} \sum_{i=2}^{n} a_{p, 1} a_{q, i} a_{j, i} a_{j, y}-Z_{p, q} S_{1, y} \\
& -\left(1-\left(\delta_{1, y}+\delta_{2, y}\right)\right) a_{p, 1} a_{q, y}+a_{p, 1} \delta_{q, 1} a_{1, y} \\
= & \sum_{i=3}^{n} \sum_{i=2}^{n} a_{p, 1} a_{q, i} a_{j, i} a_{j, y}-Z_{p, q} S_{1, y} \\
& -a_{p, 1} a_{q, y}+\delta_{1, y} a_{p, 1} a_{q, 1}+\delta_{2, y} a_{p, 1} a_{q, 2}+a_{p, 1} \delta_{q, 1} a_{1, y}
\end{aligned}
$$


Beginnend mit $\left(a_{p, 1} a_{q, 2} a_{1,2} a_{1, y}, a_{p, 1} a_{q, 2} S_{2, y}\right)$ :

$$
\begin{aligned}
& a_{p, 1} a_{q, 2} a_{1,2} a_{1, y} \\
& \stackrel{\widetilde{S}_{2, y}}{\longrightarrow}-a_{p, 1} a_{q, 2}\left(\sum_{j=2}^{n} a_{j, 2} a_{j, y}-\delta_{2, y}\right) \\
& =-\sum_{j=2}^{n} \underline{a_{p, 1} a_{q, 2} a_{j, 2}} a_{j, y}+a_{p, 1} a_{q, 2} \delta_{2, y} \\
& \stackrel{\widetilde{Z}_{p, q, j}}{\longrightarrow}-\sum_{j=2}^{n}\left(Z_{p, q, j}-Z_{p, q} a_{j, 1}\right) a_{j, y}+a_{p, 1} a_{q, 2} \delta_{2, y} \\
& =\sum_{j=2}^{n}\left(a_{p, 1} \sum_{i=3}^{n} a_{q, i} a_{j, i}-a_{p, 1} \delta_{q, j}\right) a_{j, y}+Z_{p, q} \sum_{j=2}^{n} a_{j, 1} a_{j, y} \\
& +a_{p, 1} a_{q, 2} \delta_{2, y} \\
& =\sum_{j=2}^{n}\left(a_{p, 1} \sum_{i=3}^{n} a_{q, i} a_{j, i}-a_{p, 1} \delta_{q, j}\right) a_{j, y}-Z_{p, q} S_{1, y} \\
& +Z_{p, q} \delta_{1, y}+a_{p, 1} a_{q, 2} \delta_{2, y} \\
& =\sum_{i=3}^{n} \sum_{j=2}^{n} a_{p, 1} a_{q, i} a_{j, i} a_{j, y}-Z_{p, q} S_{1, y} \\
& -\underbrace{\sum_{j=2}^{n} a_{p, 1} \delta_{q, j} a_{j, y}}_{(1-\delta 1, q) a_{p, 1} a_{q, y}}+Z_{p, q} \delta_{1, y}+a_{p, 1} a_{q, 2} \delta_{2, y} \\
& =\sum_{i=3}^{n} \sum_{j=2}^{n} a_{p, 1} a_{q, i} a_{j, i} a_{j, y}-Z_{p, q} S_{1, y} \\
& -a_{p, 1} a_{q, y}+\delta_{1, q} a_{p, 1} a_{1, y}+Z_{p, q} \delta_{1, y}+a_{p, 1} a_{q, 2} \delta_{2, y}
\end{aligned}
$$

\section{Bemerkung 2.2.13 (Symmetrie)}

Diese Rechnungen sind nach Vertauschen der Indizes identisch mit denen in 2.2.14 
2.2.14 Reduktionswege für $a_{1, p} a_{2, q} a_{2,1} a_{y, 1}$

Beginnend mit $\left(a_{1, p} a_{2, q} a_{2,1} a_{y, 1},\left(S_{p, q, 1}-S_{p, q} a_{1,1}\right) a_{y, 1}\right)$ :

$$
\begin{aligned}
& \widetilde{Z}_{p, q, 1} \underline{a_{1, p} a_{2, q} a_{2,1}} a_{y, 1} \\
& \hline \widetilde{S}_{1, y} \longrightarrow\left.-a_{1, p} \sum_{i=3}^{n} a_{i, q} a_{i, 1}+a_{1, p} \delta_{q, 1}\right) a_{y, 1}-S_{p, q} \underline{a_{1,1} a_{y, 1}} \\
& \hline \widetilde{S}_{i, y} a_{i, q} a_{i, 1} a_{y, 1}+a_{1, p} \delta_{q, 1} a_{y, 1}-S_{p, q} Z_{1, y} \\
& \longrightarrow \sum_{i=3}^{n} a_{1, p} a_{i, q}\left(\sum_{j=2}^{n} a_{i, j} a_{y, j}-\delta_{i, y}\right)+a_{1, p} \delta_{q, 1} a_{y, 1}-S_{p, q} Z_{1, y} \\
&= \sum_{i=3}^{n} \sum_{i=2}^{n} a_{1, p} a_{i, q} a_{i, j} a_{y, j}-S_{p, q} Z_{1, y} \\
&-\sum_{i=3}^{n} a_{1, p} a_{i, q} \delta_{i, y}+a_{1, p} \delta_{q, 1} a_{y, 1} \\
&= \sum_{i=3}^{n} \sum_{i=2}^{n} a_{1, p} a_{i, q} a_{i, j} a_{y, j}-S_{p, q} Z_{1, y} \\
&-\left(1-\left(\delta_{1, y}+\delta_{2, y}\right)\right) a_{1, p} a_{y, q}+a_{1, p} \delta_{q, 1} a_{y, 1} \\
&= \sum_{i=3}^{n} \sum_{i=2}^{n} a_{1, p} a_{i, q} a_{i, j} a_{y, j}-S_{p, q} Z_{1, y} \\
&-a_{1, p} a_{y, q}+\delta_{1, y} a_{1, p} a_{1, q}+\delta_{2, y} a_{1, p} a_{2, q}+a_{1, p} \delta_{q, 1} a_{y, 1}
\end{aligned}
$$


Beginnend mit $\left(a_{1, p} a_{2, q} a_{2,1} a_{y, 1}, a_{1, p} a_{2, q} Z_{2, y}\right)$ :

$$
\begin{aligned}
& a_{1, p} a_{2, q} \underline{a_{2,1} a_{y, 1}} \\
& \mid \stackrel{\widetilde{S}_{2, y}}{\longrightarrow}-a_{1, p} a_{2, q}\left(\sum_{j=2}^{n} a_{2, j} a_{y, j}-\delta_{2, y}\right) \\
& =-\sum_{j=2}^{n} \underline{a_{1, p} a_{2, q} a_{2, j}} a_{y, j}+a_{1, p} a_{2, q} \delta_{2, y} \\
& \stackrel{\widetilde{Z}_{p, q, j}}{\longrightarrow}-\sum_{j=2}^{n}\left(S_{p, q, j}-S_{p, q} a_{1, j}\right) a_{y, j}+a_{1, p} a_{2, q} \delta_{2, y} \\
& =\sum_{j=2}^{n}\left(a_{1, p} \sum_{i=3}^{n} a_{i, q} a_{i, j}-a_{1, p} \delta_{q, j}\right) a_{y, j}+S_{p, q} \sum_{j=2}^{n} a_{1, j} a_{y, j} \\
& +a_{1, p} a_{2, q} \delta_{2, y} \\
& =\sum_{j=2}^{n}\left(a_{1, p} \sum_{i=3}^{n} a_{i, q} a_{i, j}-a_{1, p} \delta_{q, j}\right) a_{y, j}-S_{p, q} Z_{1, y} \\
& +S_{p, q} \delta_{1, y}+a_{1, p} a_{2, q} \delta_{2, y} \\
& =\sum_{i=3}^{n} \sum_{j=2}^{n} a_{1, p} a_{i, q} a_{i, j} a_{y, j}-S_{p, q} Z_{1, y} \\
& -\underbrace{\sum_{j=2}^{n} a_{1, p} \delta_{q, j} a_{y, j}}_{(1-\delta 1, q) a_{1, p} a_{y, q}}+S_{p, q} \delta_{1, y}+a_{1, p} a_{2, q} \delta_{2, y} \\
& =\sum_{i=3}^{n} \sum_{j=2}^{n} a_{1, p} a_{i, q} a_{i, j} a_{y, j}-S_{p, q} Z_{1, y} \\
& -a_{1, p} a_{y, q}+\delta_{1, q} a_{1, p} a_{y, 1}+S_{p, q} \delta_{1, y}+a_{1, p} a_{2, q} \delta_{2, y}
\end{aligned}
$$

\section{Bemerkung 2.2.14 (Symmetrie)}

Diese Rechnungen sind nach Vertauschen der Indizes identisch mit denen in 2.2.13 


\subsubsection{Reduktionswege für $a_{p, 1} a_{1,2} a_{1,2}$}

Beginnend mit $\left(a_{p, 1} a_{1,2} a_{1,2}, Z_{p, 1,1}-Z_{p, 1} a_{1,1}\right)$ :

$$
\begin{aligned}
& a_{p, 1} a_{1,2} a_{1,2} \\
& \mid \stackrel{\widetilde{Z}_{p, 1,1}}{\longrightarrow}-a_{p, 1} \sum_{i=3}^{n} \underline{a_{1, i} a_{1, i}}+a_{p, 1} \delta_{1,1}+\sum_{i=2}^{n} a_{p, i} \underline{a_{1, i} a_{1,1}}-\delta_{p, 1} a_{1,1} \\
& \stackrel{\widetilde{S}_{i, i}, \widetilde{S}_{i, 1}}{\longrightarrow}-a_{p, 1} \sum_{i=3}^{n} S_{i, i}+a_{p, 1}+\sum_{i=2}^{n} a_{p, i} S_{i, 1}-\delta_{p, 1} a_{1,1} \\
& =-a_{p, 1} \sum_{i=3}^{n} S_{i, i}+\sum_{i=2}^{n} a_{p, i} S_{i, 1}+\left(1-\delta_{p, 1}\right) a_{p, 1}
\end{aligned}
$$

Beginnend mit $\left(a_{p, 1} a_{1,2} a_{1,2}, a_{p, 1} S_{2,2}\right)$ :

$$
\begin{aligned}
& a_{p, 1} \underline{a_{1,2} a_{1,2}} \mid \stackrel{\widetilde{S}_{2,2}}{\longrightarrow}-\sum_{j=2}^{n} \underline{a_{p, 1} a_{j, 2} a_{j, 2}}+a_{p, 1} \delta_{2,2} \\
& \stackrel{\widetilde{Z}_{p, j, j}}{\longrightarrow}-\sum_{j=2}^{n}\left(Z_{p, j, j}-Z_{p, j} a_{j, 1}\right)+a_{p, 1} \\
& =\sum_{j=2}^{n}\left(a_{p, 1} \sum_{i=3}^{n} a_{j, i} a_{j, i}-a_{p, 1} \delta_{j, j}\right) \\
& -\sum_{j=2}^{n}\left(\sum_{i=2}^{n} a_{p, i} a_{j, i}-\delta_{p, j}\right) a_{j, 1}+a_{p, 1} \\
& =a_{p, 1} \sum_{i=3}^{n}\left(\sum_{j=2}^{n} a_{j, i} a_{j, i}-\delta_{i, i}\right)-a_{p, 1} \\
& -\sum_{i=2}^{n} a_{p, i} \sum_{j=2}^{n}\left(a_{j, i} a_{j, 1}-0\right)+\underbrace{\sum_{j=2}^{n} \delta_{p, j} a_{j, 1}}_{=\left(1-\delta_{p, 1}\right) a_{p, 1}}+a_{p, 1} \\
& =-a_{p, 1} \sum_{i=3}^{n} S_{i, i}+\sum_{i=2}^{n} a_{p, i} S_{i, 1}+\left(1-\delta_{p, 1}\right) a_{p, 1}
\end{aligned}
$$

Bemerkung 2.2.15 (Symmetrie)

Diese Rechnungen sind nach Vertauschen der Indizes identisch mit denen in 2.2.16 


\subsubsection{Reduktionswege für $a_{1, p} a_{2,1} a_{2,1}$}

Beginnend mit $\left(a_{1, p} a_{2,1} a_{2,1}, S_{p, 1,1}-S_{p, 1} a_{1,1}\right)$ :

$$
\begin{aligned}
\mid \stackrel{\widetilde{S}_{p, 1,1}}{\longrightarrow}-a_{1, p} \sum_{i=3}^{n} \underline{a_{i, 1} a_{i, 1}}+a_{1, p} \delta_{1,1}+\sum_{i=2}^{n} a_{i, p} \underline{a_{i, 1} a_{1,1}}-\delta_{p, 1} a_{1,1} \\
\widetilde{Z}_{i, i}, \widetilde{Z}_{i, 1} \\
\stackrel{-}{\longrightarrow}-a_{1, p} \sum_{i=3}^{n} Z_{i, i}+a_{1, p}+\sum_{i=2}^{n} a_{i, p} Z_{i, 1}-\delta_{p, 1} a_{1,1} \\
=-a_{1, p} \sum_{i=3}^{n} Z_{i, i}+\sum_{i=2}^{n} a_{i, p} Z_{i, 1}+\left(1-\delta_{p, 1}\right) a_{1, p}
\end{aligned}
$$

Beginnend mit $\left(a_{1, p} a_{2,1} a_{2,1}, a_{1, p} Z_{2,2}\right)$ :

$$
\begin{aligned}
& a_{1, p} \underline{a_{2,1} a_{2,1}} \mid \stackrel{\widetilde{Z}_{2,2} \longrightarrow}{\longrightarrow}-\sum_{j=2}^{n} \frac{a_{1, p} a_{2, j} a_{2, j}}{\widetilde{S}_{p, j, j} \longrightarrow}+a_{1, p} \delta_{2,2} \\
&-\sum_{j=2}^{n}\left(S_{p, j, j}-S_{p, j} a_{1, j}\right)+a_{1, p} \\
&=\sum_{j=2}^{n}\left(a_{1, p} \sum_{i=3}^{n} a_{i, j} a_{i, j}-a_{1, p} \delta_{j, j}\right) \\
&-\sum_{j=2}^{n}\left(\sum_{i=2}^{n} a_{i, p} a_{i, j}-\delta_{p, j}\right) a_{1, j}+a_{1, p} \\
&= a_{1, p} \sum_{i=3}^{n}\left(\sum_{j=2}^{n} a_{i, j} a_{i, j}-\delta_{i, i}\right)-a_{1, p} \\
&-\sum_{i=2}^{n} a_{i, p} \sum_{j=2}^{n}\left(a_{i, j} a_{1, j}-0\right)+\underbrace{\sum_{j=2}^{n} \delta_{p, j} a_{1, j}}_{=\left(1-\delta_{p, 1}\right) a_{1, p}}+a_{1, p} \\
&=-a_{1, p} \sum_{i=3}^{n} Z_{i, i}+\sum_{i=2}^{n} a_{i, p} Z_{i, 1}+\delta_{\left(1-\delta_{p, 1}\right)}^{\left(1-a_{1, p}\right.}
\end{aligned}
$$

Bemerkung 2.2.16 (Symmetrie)

Diese Rechnungen sind nach Vertauschen der Indizes identisch mit denen in 2.2.15 
2.2.17 Reduktionswege für $a_{p, 1} a_{q, 2} a_{1,2} a_{2, y} a_{2, z}$

Beginnend mit $\left(a_{p, 1} a_{q, 2} a_{1,2} a_{2, y} a_{2, z},\left(Z_{p, q, 1}-Z_{p, q} a_{1,1}\right) a_{2, y} a_{2, z}\right)$ :

$$
\begin{aligned}
\mid \widetilde{Z}_{p, q, 1} & \frac{a_{p, 1} a_{q, 2} a_{1,2}}{\longrightarrow} a_{2, y} a_{2, z} \\
\stackrel{\widetilde{S}_{1, y, z}}{\longrightarrow} & -\left(a_{p, 1} \sum_{i=3}^{n} a_{q, i} a_{1, i}-a_{p, 1} \delta_{q, 1}\right) a_{2, y} a_{2, z}-Z_{p, q} \underline{a_{1,1} a_{2, y} a_{2, z}} \\
\hline & -\sum_{i=3}^{n} a_{p, 1} a_{q, i} \underline{a_{1, i} a_{2, y} a_{2, z}}+a_{p, 1} \delta_{q, 1} a_{2, y} a_{2, z} \\
\mid \stackrel{\widetilde{S}_{i, y, z}}{\longrightarrow} & -\sum_{i=3}^{n} a_{p, 1} a_{q, i}\left(S_{1, y, z}-S_{1, y} a_{1, z}\right) \\
& -Z_{p, q}\left(S_{1, y, z}-S_{1, y} a_{1, z}\right)+a_{p, 1} \delta_{q, 1} a_{2, y} a_{2, z}
\end{aligned}
$$

Beginnend mit $\left(a_{p, 1} a_{q, 2} a_{1,2} a_{2, y} a_{2, z}, a_{p, 1} a_{q, 2}\left(S_{2, y, z}-S_{2, y} a_{1, z}\right)\right)$ :

$$
\begin{aligned}
& a_{p, 1} a_{q, 2} \frac{a_{1,2} a_{2, y} a_{2, z}}{\widetilde{S}_{2, y, z}} \longrightarrow \\
\longrightarrow & -a_{p, 1} a_{q, 2}\left(a_{1,2} \sum_{j=3}^{n} a_{j, y} a_{j, z}-a_{1,2} \delta_{y, z}\right) \\
& +a_{p, 1} a_{q, 2}\left(\sum_{j=2}^{n} a_{j, 2} a_{j, y}-\delta_{2, y}\right) a_{1, z} \\
= & -\underline{a_{p, 1} a_{q, 2} a_{1,2}}\left(\sum_{j=3}^{n} a_{i, y} a_{i, z}-\delta_{y, z}\right) \\
& +\sum_{j=2}^{n} \frac{a_{p, 1} a_{q, 2} a_{j, 2} a_{j, y} a_{1, z}-a_{p, 1} a_{q, 2} \delta_{2, y} a_{1, z}}{\longrightarrow} \\
\widetilde{Z}_{p, q, 1}, \widetilde{Z}_{p, q, j} & -\left(Z_{p, q, 1}-Z_{p, q} a_{1,1}\right)\left(\sum_{j=3}^{n} a_{j, y} a_{j, z}-\delta_{y, z}\right) \\
& +\sum_{j=2}^{n}\left(Z_{p, q, j}-Z_{p, q} a_{j, 1}\right) a_{j, y} a_{1, z}-a_{p, 1} a_{q, 2} \delta_{2, y} a_{1, z}
\end{aligned}
$$




$$
\begin{aligned}
& \stackrel{2.2 .2}{=}-\left(a_{p, 1} \sum_{i=3}^{n} a_{q, i} S_{i, y, z}+\delta_{q, 1} a_{p, 1}\left(\sum_{j=3}^{n} a_{j, y} a_{j, z}-\delta_{y, z}\right)\right) \\
& -Z_{p, q} S_{1, y, z}+\left(\sum_{i=3}^{n} a_{p, 1} a_{q, i} S_{i, y}+\delta_{y, 1} a_{p, 1} a_{q, 1} .\right. \\
& \left.+\delta_{y, 2} a_{p, 1} a_{q, 2}-\delta_{q, 1} a_{p, 1} a_{1, y}\right) a_{1, z} \\
& -\sum_{j=2}^{n} Z_{p, q} a_{j, 1} a_{j, y} a_{1, z}-a_{p, 1} a_{q, 2} \delta_{2, y} a_{1, z} \\
& =-\sum_{i=3}^{n} a_{p, 1} a_{q, i}\left(S_{i, y, z}-S_{i, y} a_{1, z}\right) \\
& -\delta_{q, 1} a_{p, 1}\left(\sum_{j=3}^{n} a_{j, y} a_{j, z}-\delta_{y, z}\right)-\delta_{q, 1} a_{p, 1} \underline{a_{1, y} a_{1, z}} \\
& +\left(\delta_{y, 1} \underline{a_{p, 1} a_{q, 1}}-\sum_{j=2}^{n} Z_{p, q} a_{j, 1} a_{j, y}\right) a_{1, z}-Z_{p, q} S_{1, y, z} \\
& \mid \stackrel{\widetilde{Z}_{p, q}, \widetilde{S}_{p, q}}{\longrightarrow}-\sum_{i=3}^{n} a_{p, 1} a_{q, i}\left(S_{i, y, z}-S_{i, y} a_{1, z}\right) \\
& -\delta_{q, 1} a_{p, 1}\left(\sum_{j=3}^{n} a_{j, y} a_{j, z}-\delta_{y, z}\right)-\delta_{q, 1} a_{p, 1} S_{p, q} \\
& +\left(\delta_{y, 1} Z_{p, q}-\sum_{j=2}^{n} Z_{p, q} a_{j, 1} a_{j, y}\right) a_{1, z}-Z_{p, q} S_{1, y, z} \\
& =-\sum_{i=3}^{n} a_{p, 1} a_{q, i}\left(S_{i, y, z}-S_{i, y} a_{1, z}\right)+a_{p, 1} \delta_{q, 1} a_{2, y} a_{2, z} \\
& -Z_{p, q}\left(S_{1, y, z}-S_{1, y} a_{1, z}\right)
\end{aligned}
$$

\section{Bemerkung 2.2.17 (Symmetrie)}

Diese Rechnungen sind nach Vertauschen der Indizes identisch mit denen in 2.2.18 
2.2.18 Reduktionswege für $a_{1, p} a_{2, q} a_{2,1} a_{y, 2} a_{z, 2}$

Beginnend mit $\left(a_{1, p} a_{2, q} a_{2,1} a_{y, 2} a_{z, 2},\left(S_{p, q, 1}-S_{p, q} a_{1,1}\right) a_{y, 2} a_{z, 2}\right)$ :

$$
\begin{aligned}
& \underline{a_{1, p} a_{2, q} a_{2,1}} a_{y, 2} a_{z, 2} \\
& \mid \stackrel{\widetilde{Z}_{p, q, 1}}{\longrightarrow}-\left(a_{1, p} \sum_{i=3}^{n} a_{i, q} a_{i, 1}-a_{1, p} \delta_{q, 1}\right) a_{y, 2} a_{z, 2}-S_{p, q} \underline{a_{1,1} a_{y, 2} a_{z, 2}} \\
& \mid \stackrel{\widetilde{S}_{1, y, z}}{\longrightarrow}-\sum_{i=3}^{n} a_{1, p} a_{i, q} \underline{a_{i, 1} a_{y, 2} a_{z, 2}}+a_{1, p} \delta_{q, 1} a_{y, 2} a_{z, 2} \\
& \text { - } S_{p, q}\left(Z_{1, y, z}-Z_{1, y} a_{z, 1}\right) \\
& \mid \stackrel{\widetilde{S}_{i, y, z}}{\longrightarrow}-\sum_{i=3}^{n} a_{1, p} a_{i, q}\left(Z_{i, y, z}-Z_{i, y} a_{z, 1}\right)+a_{1, p} \delta_{q, 1} a_{y, 2} a_{z, 2} \\
& -S_{p, q}\left(Z_{1, y, z}-Z_{1, y} a_{z, 1}\right)
\end{aligned}
$$

Beginnend mit $\left(a_{1, p} a_{2, q} a_{2,1} a_{y, 2} a_{z, 2}, a_{1, p} a_{2, q}\left(Z_{2, y, z}-Z_{2, y} a_{z, 1}\right)\right)$ :

$$
\begin{aligned}
& a_{1, p} a_{2, q} \frac{a_{2,1} a_{y, 2} a_{z, 2}}{\widetilde{S}_{2, y, z}} \rightarrow \\
\longrightarrow & -a_{1, p} a_{2, q}\left(a_{2,1} \sum_{j=3}^{n} a_{y, j} a_{z, j}-a_{2,1} \delta_{y, z}\right) \\
& +a_{1, p} a_{2, q}\left(\sum_{j=2}^{n} a_{2, j} a_{y, j}-\delta_{2, y}\right) a_{z, 1} \\
= & -\underline{a_{1, p} a_{2, q} a_{2,1}}\left(\sum_{j=3}^{n} a_{y, i} a_{z, i}-\delta_{y, z}\right) \\
& +\sum_{j=2}^{n} \frac{a_{1, p} a_{2, q} a_{2, j} a_{y, j} a_{z, 1}-a_{1, p} a_{2, q} \delta_{2, y} a_{z, 1}}{\longrightarrow} \\
\widetilde{Z}_{p, q, 1}, \widetilde{Z}_{p, q, j} & -\left(S_{p, q, 1}-S_{p, q} a_{1,1}\right)\left(\sum_{j=3}^{n} a_{y, j} a_{z, j}-\delta_{y, z}\right) \\
& +\sum_{j=2}^{n}\left(S_{p, q, j}-S_{p, q} a_{1, j}\right) a_{y, j} a_{z, 1}-a_{1, p} a_{2, q} \delta_{2, y} a_{z, 1}
\end{aligned}
$$




$$
\begin{aligned}
& \stackrel{2.2 .2}{=}-\left(a_{1, p} \sum_{i=3}^{n} a_{i, q} Z_{i, y, z}+\delta_{q, 1} a_{1, p}\left(\sum_{j=3}^{n} a_{y, j} a_{z, j}-\delta_{y, z}\right)\right) \\
& -S_{p, q} Z_{1, y, z}+\left(\sum_{i=3}^{n} a_{p, 1} a_{q, i} S_{i, y}+\delta_{y, 1} a_{p, 1} a_{q, 1} .\right. \\
& \left.+\delta_{y, 2} a_{p, 1} a_{q, 2}-\delta_{q, 1} a_{p, 1} a_{1, y}\right) a_{z, 1} \\
& -\sum_{j=2}^{n} S_{p, q} a_{1, j} a_{y, j} a_{z, 1}-a_{1, p} a_{2, q} \delta_{2, y} a_{z, 1} \\
& =-\sum_{i=3}^{n} a_{1, p} a_{i, q}\left(Z_{i, y, z}-Z_{i, y} a_{z, 1}\right) \\
& -\delta_{q, 1} a_{1, p}\left(\sum_{j=3}^{n} a_{y, j} a_{z, j}-\delta_{y, z}\right)-\delta_{q, 1} a_{1, p} \underline{a_{y, 1} a_{z, 1}} \\
& +\left(\delta_{y, 1} \underline{a_{1, p} a_{1, q}}-\sum_{j=2}^{n} S_{p, q} a_{1, j} a_{y, j}\right) a_{z, 1}-S_{p, q} Z_{1, y, z} \\
& \mid \stackrel{\widetilde{Z}_{p, q}, \widetilde{S}_{p, q}}{\longrightarrow}-\sum_{i=3}^{n} a_{1, p} a_{i, q}\left(Z_{i, y, z}-Z_{i, y} a_{z, 1}\right) \\
& -\delta_{q, 1} a_{1, p}\left(\sum_{j=3}^{n} a_{y, j} a_{z, j}-\delta_{y, z}\right)-\delta_{q, 1} a_{1, p} Z_{p, q} \\
& +\left(\delta_{y, 1} S_{p, q}-\sum_{j=2}^{n} S_{p, q} a_{1, j} a_{y, j}\right) a_{z, 1}-S_{p, q} Z_{1, y, z} \\
& =-\sum_{i=3}^{n} a_{1, p} a_{i, q}\left(Z_{i, y, z}-Z_{i, y} a_{z, 1}\right)+a_{1, p} \delta_{q, 1} a_{y, 2} a_{z, 2} \\
& -S_{p, q}\left(Z_{1, y, z}-Z_{1, y} a_{z, 1}\right)
\end{aligned}
$$

\section{Bemerkung 2.2.18 (Symmetrie)}

Diese Rechnungen sind nach Vertauschen der Indizes identisch mit denen in 2.2.17 
2.2.19 Reduktionswege für $a_{p, 1} a_{1,2} a_{2,2} a_{2, z}$

Beginnend mit $\left(a_{p, 1} a_{1,2} a_{2,2} a_{2, z},\left(Z_{p, 1,2}-Z_{p, 1} a_{2,1}\right) a_{2, z}\right)$ :

$$
\begin{aligned}
\underline{a p, 1}_{p, 2} a_{2,2} a_{2, z} \mid \stackrel{\widetilde{Z}_{p, 1,2}}{\longrightarrow} & -\left(a_{p, 1} \sum_{i=3}^{n} a_{1, i} a_{2, i}-a_{p, 1} \delta_{1,2}\right) a_{2, z} \\
& +\left(\sum_{i=2}^{n} a_{p, i} a_{1, i}-\delta_{p, 1}\right) a_{2,1} a_{2, z} \\
= & -\sum_{i=3}^{n} a_{p, 1} \underline{a_{1, i} a_{2, i} a_{2, z}} \\
& +\sum_{i=2}^{n} a_{p, i} \underline{a_{1, i} a_{2,1} a_{2, z}}-\delta_{p, 1} a_{2,1} a_{2, z} \\
\widetilde{S}_{i, i, z}, \widetilde{S}_{i, 1, z} \longrightarrow & -\sum_{i=3}^{n} a_{p, 1}\left(S_{i, i, z}-S_{i, i} a_{1, z}\right) \\
& +\sum_{i=2}^{n} a_{p, i}\left(S_{i, 1, z}-S_{i, 1} a_{1, z}\right)-\delta_{p, 1} a_{2,1} a_{2, z}
\end{aligned}
$$

Beginnend mit $\left(a_{p, 1} a_{1,2} a_{2,2} a_{2, z}, a_{p, 1}\left(S_{2,2, z}-S_{2,2} a_{1, z}\right)\right)$ :

$$
\begin{aligned}
\widetilde{S}_{2,2, z} & a_{p, 1} \\
\stackrel{a_{1,2} a_{2,2} a_{2, z}}{\longrightarrow} & -a_{p, 1}\left(a_{1,2} \sum_{j=3}^{n} a_{j, 2} a_{j, z}-a_{1,2} \delta_{2, z}\right) \\
& +a_{p, 1}\left(\sum_{j=2}^{n} a_{j, 2} a_{j, 2}-\delta_{2,2}\right) a_{1, z} \\
= & -\sum_{j=3}^{n} \underline{a_{p, 1} a_{1,2} a_{j, 2}} a_{j, z}+a_{p, 1} a_{1,2} \delta_{2, z} \\
& +\sum_{j=2}^{n} \underline{a_{p, 1} a_{j, 2} a_{j, 2}} a_{1, z}-a_{p, 1} a_{1, z}
\end{aligned}
$$




$$
\begin{aligned}
& \mid \stackrel{\widetilde{Z}_{p, 1, j}, \widetilde{Z}_{p, j, j}}{\longrightarrow}-\sum_{j=3}^{n}\left(Z_{p, 1, j}-Z_{p, 1} a_{j, 1}\right) a_{j, z}-\left(1-\delta_{2, z}\right) a_{p, 1} a_{1, z} \\
& +\sum_{j=2}^{n}\left(Z_{p, j, j}-Z_{p, j} a_{j, 1}\right) a_{1, z} \\
& \stackrel{2.2 .2}{=}-\left(\sum_{i=3}^{n} a_{p, 1} S_{i, i, z}-\left(1-\delta_{1, z}-\delta_{2, z}\right) a_{p, 1} a_{1, z}\right) \\
& +\left(\sum_{i=2}^{n} a_{p, i} S_{i, 1, z}-\sum_{i=2}^{n} a_{p, i} a_{1, i} \delta_{1, z}+\sum_{j=3}^{n} \delta_{p, 1} a_{j, 1} a_{j, z}\right) \\
& -\left(1-\delta_{2, z}\right) a_{p, 1} a_{1, z}+\left(\sum_{i=3}^{n} a_{p, 1} S_{i, i}+a_{p, 1}\right) a_{1, z} \\
& -\left(\sum_{i=2}^{n} a_{p, i} S_{i, 1}+\left(\delta_{1,1}-\delta_{p, 1}\right) a_{p, 1}\right) a_{1, z} \\
& =-\sum_{i=3}^{n} a_{p, 1}\left(S_{i, i, z}-S_{i, i} a_{1, z}\right)+\sum_{i=2}^{n} a_{p, i}\left(S_{i, 1, z}-S_{i, 1} a_{1, z}\right) \\
& -\delta_{p, 1} a_{2,1} a_{2, z}-\delta_{1, z}\left(\underline{\left.\sum_{i=2}^{n} a_{p, i} a_{1, i}+a_{p, 1} a_{1,1}\right)}\right. \\
& +\delta_{p, 1}\left(\sum_{j=2}^{n} a_{j, 1} a_{j, z}+\underline{a_{1,1} a_{1, z}}\right) \\
& \mid \stackrel{\widetilde{Z}_{p, 1}, \widetilde{Z}_{1, z}}{\longrightarrow}-\sum_{i=3}^{n} a_{p, 1}\left(S_{i, i, z}-S_{i, i} a_{1, z}\right)+\sum_{i=2}^{n} a_{p, i}\left(S_{i, 1, z}-S_{i, 1} a_{1, z}\right) \\
& -\delta_{p, 1} a_{2,1} a_{2, z}-\delta_{1, z}\left(\sum_{i=2}^{n} a_{p, i} a_{1, i}+Z_{p, 1}\right) \\
& +\delta_{p, 1}\left(\sum_{j=2}^{n} a_{j, 1} a_{j, z}+Z_{1, z}\right) \\
& =-\sum_{i=3}^{n} a_{p, 1}\left(S_{i, i, z}-S_{i, i} a_{1, z}\right) \\
& +\sum_{i=2}^{n} a_{p, i}\left(S_{i, 1, z}-S_{i, 1} a_{1, z}\right)-\delta_{p, 1} a_{2,1} a_{2, z}
\end{aligned}
$$

\section{Bemerkung 2.2.19 (Symmetrie)}

Diese Rechnungen sind nach Vertauschen der Indizes identisch mit denen in 2.2.20 
2.2.20 Reduktionswege für $a_{1, p} a_{2,1} a_{2,2} a_{z, 2}$

Beginnend mit $\left(a_{1, p} a_{2,1} a_{2,2} a_{z, 2},\left(S_{p, 1,2}-S_{p, 1} a_{1,2}\right) a_{z, 2}\right)$ :

$$
\begin{aligned}
\underline{a_{1, p} a_{2,1} a_{2,2}} a_{z, 2} \mid \stackrel{\widetilde{Z}_{p, 1,2}}{\longrightarrow} & -\left(a_{1, p} \sum_{i=3}^{n} a_{i, 1} a_{i, 2}-a_{1, p} \delta_{1,2}\right) a_{z, 2} \\
& +\left(\sum_{i=2}^{n} a_{i, p} a_{i, 1}-\delta_{p, 1}\right) a_{1,2} a_{z, 2} \\
= & -\sum_{i=3}^{n} a_{1, p} \underline{a_{i, 1} a_{i, 2} a_{z, 2}} \\
& +\sum_{i=2}^{n} a_{i, p} \underline{a_{i, 1} a_{1,2} a_{z, 2}}-\delta_{p, 1} a_{1,2} a_{z, 2} \\
\mid \widetilde{S}_{i, i, z}, \widetilde{S}_{i, 1, z} & -\sum_{i=3}^{n} a_{1, p}\left(Z_{i, i, z}-Z_{i, i} a_{z, 1}\right) \\
& +\sum_{i=2}^{n} a_{i, p}\left(Z_{i, 1, z}-Z_{i, 1} a_{z, 1}\right)-\delta_{p, 1} a_{1,2} a_{z, 2}
\end{aligned}
$$

Beginnend mit $\left(a_{1, p} a_{2,1} a_{2,2} a_{z, 2}, a_{1, p}\left(Z_{2,2, z}-Z_{2,2} a_{z, 1}\right)\right)$ :

$$
\begin{aligned}
\widetilde{S}_{2,2, z} \longrightarrow & -a_{1, p}\left(a_{2,1} \sum_{j=3}^{n} a_{2, j} a_{z, j}-a_{2,1} \delta_{2, z}\right) \\
& +a_{1, p}\left(\sum_{j=2}^{n} a_{2, j} a_{2, j}-\delta_{2,2}\right) a_{z, 1} \\
= & -\sum_{j=3}^{n} \underline{a_{1, p} a_{2,1} a_{2, j}} a_{z, j}+a_{1, p} a_{2,1} \delta_{2, z} \\
& +\sum_{j=2}^{n} \underline{a_{1, p} a_{2, j} a_{2, j}} a_{z, 1}-a_{1, p} a_{z, 1}
\end{aligned}
$$




$$
\begin{aligned}
& \mid \stackrel{\widetilde{Z}_{p, 1, j}, \widetilde{Z}_{p, j, j}}{\longrightarrow}-\sum_{j=3}^{n}\left(S_{p, 1, j}-S_{p, 1} a_{1, j}\right) a_{z, j}-\left(1-\delta_{2, z}\right) a_{1, p} a_{z, 1} \\
& +\sum_{j=2}^{n}\left(S_{p, j, j}-S_{p, j} a_{1, j}\right) a_{z, 1} \\
& \stackrel{2.2 .2}{=}-\left(\sum_{i=3}^{n} a_{1, p} Z_{i, i, z}-\left(1-\delta_{1, z}-\delta_{2, z}\right) a_{1, p} a_{z, 1}\right) \\
& +\left(\sum_{i=2}^{n} a_{i, p} Z_{i, 1, z}-\sum_{i=2}^{n} a_{i, p} a_{i, 1} \delta_{1, z}+\sum_{j=3}^{n} \delta_{p, 1} a_{1, j} a_{z, j}\right) \\
& -\left(1-\delta_{2, z}\right) a_{1, p} a_{z, 1}+\left(\sum_{i=3}^{n} a_{1, p} Z_{i, i}+a_{1, p}\right) a_{z, 1} \\
& -\left(\sum_{i=2}^{n} a_{i, p} Z_{i, 1}+\left(\delta_{1,1}-\delta_{p, 1}\right) a_{1, p}\right) a_{z, 1} \\
& =-\sum_{i=3}^{n} a_{1, p}\left(Z_{i, i, z}-Z_{i, i} a_{z, 1}\right)+\sum_{i=2}^{n} a_{i, p}\left(Z_{i, 1, z}-Z_{i, 1} a_{z, 1}\right) \\
& -\delta_{p, 1} a_{1,2} a_{z, 2}-\delta_{1, z}\left(\underline{\sum_{i=2}^{n} a_{i, p} a_{i, 1}+a_{1, p} a_{1,1}}\right) \\
& +\delta_{p, 1}\left(\sum_{j=2}^{n} a_{1, j} a_{z, j}+\underline{a_{1,1} a_{z, 1}}\right) \\
& \mid \stackrel{\widetilde{S}_{p, 1}, \widetilde{S}_{1, z}}{\longrightarrow}-\sum_{i=3}^{n} a_{1, p}\left(Z_{i, i, z}-Z_{i, i} a_{z, 1}\right)+\sum_{i=2}^{n} a_{i, p}\left(Z_{i, 1, z}-Z_{i, 1} a_{z, 1}\right) \\
& -\delta_{p, 1} a_{1,2} a_{z, 2}-\delta_{1, z}\left(\sum_{i=2}^{n} a_{i, p} a_{i, 1}+S_{p, 1}\right) \\
& +\delta_{p, 1}\left(\sum_{j=2}^{n} a_{1, j} a_{z, j}+S_{1, z}\right) \\
& =-\sum_{i=3}^{n} a_{1, p}\left(Z_{i, i, z}-Z_{i, i} a_{z, 1}\right) \\
& +\sum_{i=2}^{n} a_{i, p}\left(Z_{i, 1, z}-Z_{i, 1} a_{z, 1}\right)-\delta_{p, 1} a_{1,2} a_{z, 2}
\end{aligned}
$$

\section{Bemerkung 2.2.20 (Symmetrie)}

Diese Rechnungen sind nach Vertauschen der Indizes identisch mit denen in 2.2.19 
2.2.21 Reduktionswege für $a_{1,1} a_{2,2} a_{2,2}$

Beginnend mit $\left(a_{1,1} a_{2,2} a_{2,2}, Z_{1,2,2}-Z_{1,2} a_{2,1}\right)$ :

$$
\begin{aligned}
& \underline{a_{1,1} a_{2,2} a_{2,2}} \\
& \mid \stackrel{\widetilde{Z}_{1,2,2}}{\longrightarrow} Z_{1,2,2}-Z_{1,2} a_{2,1} \\
& =-\left(a_{1,1} \sum_{i=3}^{n} a_{2, i} a_{2, i}-a_{1,1} \delta_{2,2}\right) \\
& +\left(\sum_{i=2}^{n} a_{1, i} a_{2, i}-\delta_{1,2}\right) a_{2,1} \\
& =-\sum_{i=3}^{n} \underline{a_{1,1} a_{2, i} a_{2, i}}+a_{1,1}-\sum_{i=2}^{n} \underline{a_{1, i} a_{2, i} a_{2,1}} \\
& \stackrel{\widetilde{S}_{1, i, i}, \widetilde{S}_{i, i, 1}}{\longrightarrow}-\sum_{i=3}^{n}\left(S_{1, i, i}-S_{1, i} a_{1, i}\right)+a_{1,1}-\sum_{i=2}^{n}\left(S_{i, i, 1}-S_{i, i} a_{1,1}\right) \\
& =-\sum_{i=3}^{n}\left(a_{1,1} \sum_{j=3}^{n} a_{j, i} a_{j, i}-a_{1,1} \delta_{i, i}\right) \\
& -\sum_{i=3}^{n}\left(\sum_{j=2}^{n} a_{j, 1} a_{j, i}-\delta_{1, i}\right) a_{1, i}+a_{1,1} \\
& -\sum_{i=2}^{n}\left(a_{1, i} \sum_{j=3}^{n} a_{j, i} a_{j, 1}-a_{1, i} \delta_{i, 1}\right) \\
& +\sum_{i=2}^{n}\left(\sum_{j=2}^{n} a_{j, i} a_{j, i}-\delta_{i, i}\right) a_{1,1} \\
& =-\sum_{i=3}^{n}\left(a_{1,1} \sum_{j=3}^{n} a_{j, i} a_{j, i}-a_{1,1} \delta_{i, i}\right)-\sum_{i=3}^{n} \sum_{j=2}^{n} a_{j, 1} a_{j, i} a_{1, i} \\
& +a_{1,1}-\sum_{i=2}^{n} \sum_{j=3}^{n} a_{1, i} a_{j, i} a_{j, 1} \\
& +\sum_{i=2}^{n}\left(\sum_{j=2}^{n} a_{j, i} a_{j, i}-\delta_{i, i}\right) a_{1,1}
\end{aligned}
$$


Beginnend mit $\left(a_{1,1} a_{2,2} a_{2,2}, S_{1,2,2}-S_{1,2} a_{1,2}\right)$ :

$$
\begin{aligned}
& \frac{a_{1,1} a_{2,2} a_{2,2}}{\widetilde{S}_{1,2,2}} S_{1,2,2}-S_{1,2} a_{1,2} \\
= & -\left(a_{1,1} \sum_{j=3}^{n} a_{j, 2} a_{j, 2}-a_{1,1} \delta_{2,2}\right) \\
& +\left(\sum_{j=2}^{n} a_{j, 1} a_{j, 2}-\delta_{1,2}\right) a_{1,2} \\
\widetilde{Z}_{1, j, j}, \widetilde{Z}_{j, j, 1} \rightarrow & -\sum_{j=3}^{n}\left(Z_{1, j, j}-Z_{1, j} a_{j, 1}\right)+a_{1,1}+\sum_{j=2}^{n}\left(Z_{j, j} a_{j, 2}+a_{1,1}+\sum_{j=2}^{n} a_{j, 1} a_{j, 2} a_{1,2} a_{1,1}\right) \\
& +\sum_{i=2}^{n}\left(\sum_{j=2}^{n} a_{j, i} a_{j, i}-\delta_{i, i}\right) a_{1,1} \\
= & -\sum_{j=3}^{n} Z_{1, j, j}-\sum_{j=3}^{n}\left(\sum_{i=2}^{n} a_{1, i} a_{j, i}-\delta_{1, j}\right) a_{j, 1} \\
& +a_{1,1}-\sum_{j=2}^{n}\left(a_{j, 1}^{n} \sum_{i=3}^{n} a_{j, i} a_{1, i}-a_{j, 1} \delta_{j, 1}\right)-\sum_{j=2}^{n} Z_{j, j} a_{1,1} a_{j, 1} \\
= & -\sum_{i=3}^{n}\left(a_{1,1} \sum_{j=3}^{n} a_{j, i} a_{j, i}-a_{1,1} \delta_{i, i}\right)-\sum_{i=3}^{n} \sum_{j=2}^{n} a_{j, 1} a_{j, i} a_{1, i}
\end{aligned}
$$




\subsection{Lineare Basis für $\mathcal{A}(2)$}

In diesem Abschnitt werden wir für den Fall $\mathcal{A}(2)$ einen Automaten angeben, der prüft, ob ein Wort ein Basiselement ist. Nach Bemerkung 1.2.43 bilden die Wörter, die sich nicht reduzieren lassen, eine Basis.

Dazu betrachten wir zunächst die folgende Tabelle. Sie enthält die linke Seite aller Reduzierungsregeln aus $r_{\mathcal{A}(n)}$ aus Satz 2.1.3 für $n=2$, also die Teilwörter, die reduziert werden können.

\begin{tabular}{|c|c|c|c|c|}
\hline & $\widetilde{Z}_{p, q}$ & $\widetilde{S}_{p, q}$ & $\widetilde{Z}_{p, q, r}$ \\
$a_{p, 1} a_{q, 1}$ & $a_{1, p} a_{1, q}$ & $a_{p, 1} a_{q, 2} a_{r, 2}$ & $\begin{array}{c}\widetilde{S}_{p, q, r} \\
a_{1, p} a_{2, q} a_{2, r}\end{array}$ \\
\hline$p=1, q=1, r=1$ & $a_{1,1} a_{1,1}$ & $a_{1,1} a_{1,1}$ & $a_{1,1} a_{1,2} a_{1,2}$ & $a_{1,1} a_{2,1} a_{2,1}$ \\
\hline$p=1, q=2, r=1$ & $a_{1,1} a_{2,1}$ & $a_{1,1} a_{1,2}$ & $a_{1,1} a_{2,2} a_{1,2}$ & $a_{1,1} a_{2,2} a_{2,1}$ \\
\hline$p=2, q=1, r=1$ & $a_{2,1} a_{1,1}$ & $a_{1,2} a_{1,1}$ & $a_{2,1} a_{1,2} a_{1,2}$ & $a_{1,2} a_{2,1} a_{2,1}$ \\
\hline$p=2, q=2, r=1$ & $a_{2,1} a_{2,1}$ & $a_{1,2} a_{1,2}$ & $a_{2,1} a_{2,2} a_{1,2}$ & $a_{1,2} a_{2,2} a_{2,1}$ \\
\hline$p=1, q=1, r=2$ & & & $a_{1,1} a_{1,2} a_{2,2}$ & $a_{1,1} a_{2,1} a_{2,2}$ \\
\hline$p=1, q=2, r=2$ & & & $a_{1,1} a_{2,2} a_{2,2}$ & $a_{1,1} a_{2,2} a_{2,2}$ \\
\hline$p=2, q=1, r=2$ & & & $a_{2,1} a_{1,2} a_{2,2}$ & $a_{1,2} a_{2,1} a_{2,2}$ \\
\hline$p=2, q=2, r=2$ & & & $a_{2,1} a_{2,2} a_{2,2}$ & $a_{1,2} a_{2,2} a_{2,2}$ \\
\hline
\end{tabular}

Um zu prüfen ob ein Wort $w$ unreduzierbar ist, kann man wie folgt vorgehen. Zuerst überprüft man, ob es eine Regel in der obigen Tabelle gibt, die mit dem ersten Buchstaben von $w$ beginnt. Da die Regeln höchstens Wörter aus drei Buchstaben reduzieren, reicht es, nur die nächsten zwei Buchstaben zu betrachten. Falls es keine entsprechende Regel gibt, wiederholt man das Verfahren vom nächsten Buchstaben aus, solange bis man am Wortende angelangt ist.

Man kann auch andersherum vorgehen. Dazu schreiben wir alle unreduzierbaren Teilwörter, die aus drei Buchstaben bestehen, in eine neue Tabelle. Sie erhält man aus obiger Tabelle indem man in jede Spalte die Wörter schreibt, die mit dem entsprechenden Buchstaben beginnen, und nicht in der obigen Tabelle vorkommen. 
Tabelle der unreduzierbaren Wörter

\begin{tabular}{|c|c|c|c|c|}
\hline & $a_{1,1}$ & $a_{1,2}$ & $a_{2,1}$ & $a_{2,2}$ \\
\hline & & & & \\
$a_{1,1}$ & & & & $a_{2,2} a_{1,1} \boldsymbol{a}_{\mathbf{2 , 2}}$ \\
& & & & \\
\hline & & & & \\
$a_{1,2}$ & & & $a_{2,1} a_{1,2} \boldsymbol{a}_{\mathbf{2 , 1}}$ & $a_{2,2} a_{1,2} \boldsymbol{a}_{\mathbf{2 , 1}}$ \\
$a_{2,1} a_{1,2} \boldsymbol{a}_{\mathbf{2}, \mathbf{2}}$ \\
\end{tabular}

Um festzustellen, ob ein Wort unreduzierbar ist, können wir nun vom ersten Buchstaben aus die nächsten zwei Buchstaben betrachten und überprüfen, ob das entsprechende Teilwort in der neuen Tabelle vorkommt. Falls es vorkommt wiederholen wir das Verfahren vom nächsten Buchstaben aus, solange bis wir am Wortende angelangt sind.

Bemerkung 2.3.1 $\left(a_{2,2}\right)$

Es gibt in der ersten Tabelle kein Wort, das mit $a_{2,2}$ beginnt. Es können also am Beginn eines unreduzierbaren Wortes beliebig viele $a_{2,2}$ stehen. Daher können wir die letzte Spalte in der neuen Tabelle ignorieren.

Wir wollen nun diese Verfahren durch einen endlichen Automaten abbilden.

Für ein Wort beginnt man in einem Startknoten und wandert jeweils entsprechend des nächsten Buchstaben über Pfeile zu einem neuen Knoten. Falls dieser Knoten nicht der Knoten „reduzierbar“ ist, so ist das Wort unreduzierbar. 


\section{Konstruktion}

Wir starten mit einem Startknoten $S$ und einem Knoten „reduzierbar“, sowie mit einem Knoten für jedes nicht leere Feld der Tabelle, benannt nach der Spalte und der Zeile. Außerdem fügen wir Knoten für jeden Buchstaben hinzu, sowie entsprechende Pfeile vom Startknoten ausgehend.

Wegen Bemerkung 2.3.1 können wir jeweils folgende Knoten zusammenfassen:

- , $a_{2,2}$ “, , , $a_{2,2} a_{2,2}$ “ und , $S$ “",

- , $a_{1,1}$ “ und , , $a_{2,2} a_{1,1}$ “,

- ,, $a_{1,2}$ “ und , $a_{2,2} a_{1,2}$ “,

- , $a_{2,1}$ “ und , $a_{2,2} a_{2,1}$ “.

Für jeden Knoten, der einem Feld in der Tabelle entspricht, fügen wir für jeden Eintrag einen Pfeil, benannt nach dem fett gedrucken Buchstaben, zum Knoten der letzten zwei Buchstaben des Eintrages ein. Als nächstes fügen wir von jedem Knoten für alle fehlenden Buchstaben Pfeile zum Knoten ,unreduzierbar" hinzu.

Sei beispielsweise der erste Buchstabe ein $a_{1,1}$, dann gibt es gemäß der Tabelle der unreduzierbaren Wörter nur zwei weiterführende Knoten „, $a_{1,1}$ “, falls ein $a_{2,2}$ folgt und „reduzierbar“ sonst.

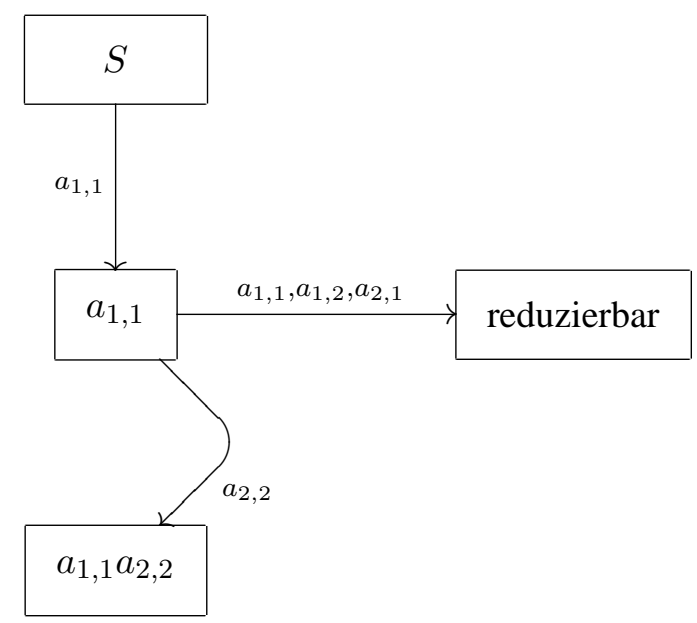

Alle Pfeile aus dem Knoten „reduzierbar“ führen wieder in diesen Knoten zurück. Da wir uns nur für die unreduzierbaren Wörter interessieren, lassen wir den Knoten „reduzierbar“ und die zu ihm führenden Pfeile im Folgenden weg und interpretieren fehlende Pfeile als Pfeile zu diesem Knoten.

So erhalten wir aus der Tabelle der unreduzierbaren Wörter den auf folgender Seite stehenden endlichen Automaten. 


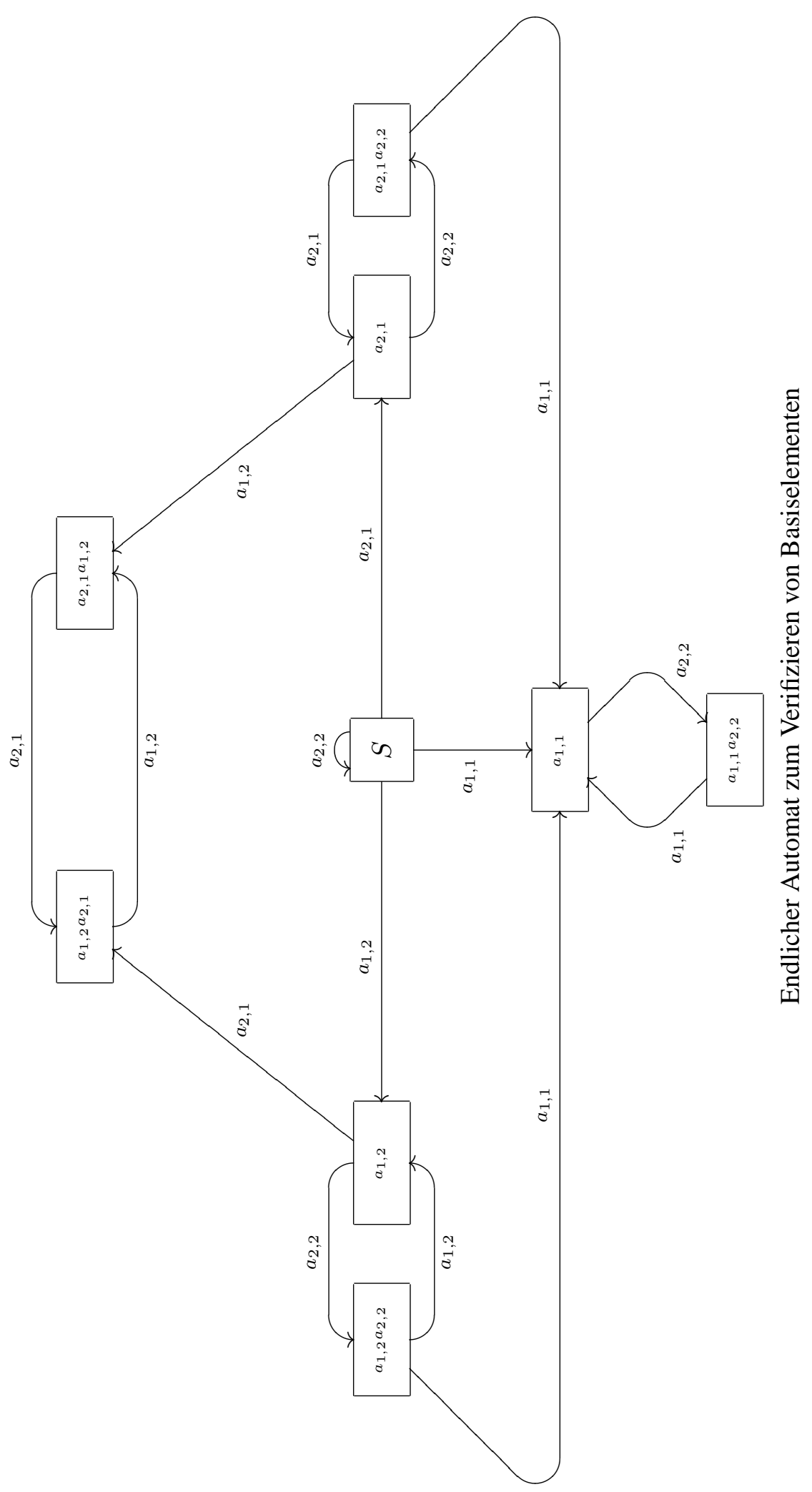


Kapitel 3

\section{Projektive Auflösungen}




\subsection{Auflösung von $\mathcal{A}(n)$ als Bimodul}

In diesem Kapitel konstruieren wir für $n \geq 3$ eine projektive Auflösung für die orthogonale freie Quantengruppe $\mathcal{A}(n)$ als $\mathcal{A}(n) \otimes \mathcal{A}^{o p}(n)$-Modul.

Dazu sei $\mathcal{A}^{e}(n):=\mathcal{A}(n) \otimes_{\mathbb{K}} \mathcal{A}^{o p}(n)$, wobei $\mathcal{A}^{o p}(n)$ die Algebra mit vertauschter Multiplikation ist. Die Elemente in $\mathcal{A}^{e}(n)$ sind erzeugt von Elementen der Form $a \otimes b$, wobei $a, b \in \mathcal{A}(n)$. Um zu verdeutlichen, dass wir $\mathcal{A}^{e}(n)$ als $\mathcal{A}(n)$-Bimodul betrachten, schreiben wir anstelle von $a \otimes b$ oft auch $a e b$, wobei $\boldsymbol{e}$ der Erzeuger des Moduls sein soll. Im Folgenden schreiben wir die Erzeuger der Moduln stets fett gedruckt.

Wir wollen nun zeigen, dass die folgende Sequenz exakt ist:

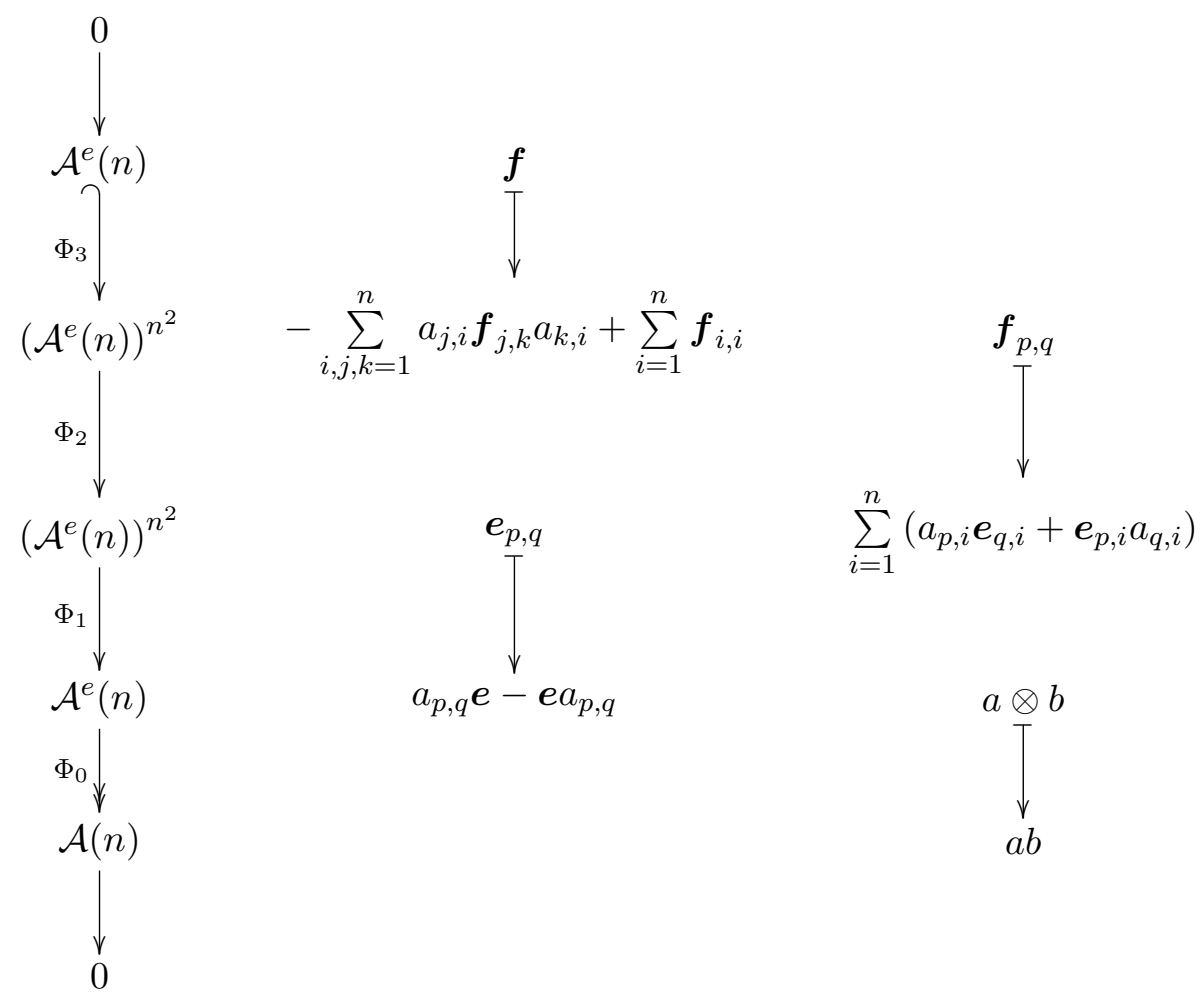

Die Abbildung $\Phi_{0}$ ist die übliche Multiplikationsabbildung. Mit $\Phi_{0}(a \otimes 1)=a$ sehen wir, dass sie surjektiv ist. In [Pie82] wird gezeigt, dass für jede Algebra mit 1 der Kern der Multiplikationsabbildung von $a \otimes 1-1 \otimes a$ aufgespannt wird, wobei $a$ über die gesamte Algebra läuft. In Abschnitt 10.2. in [Pie82] wird sogar gezeigt, dass

$$
\operatorname{Kern}\left(\Phi_{0}\right)=\sum_{a \in \text { Erzeuger }}(a \otimes 1-1 \otimes a) \times \mathcal{A}^{e}(n) .
$$

Somit ist auch die Exaktheit an der zweiten Stelle bewiesen. Im Folgenden betrachten wir nur die anderen Stellen. 
Wenn wir die Erzeuger der Moduln und der Algebra $\mathcal{A}(n)$ als generische Matrizen $\boldsymbol{E}:=\left(\boldsymbol{e}_{p, q}\right)_{p, q=1 \ldots n}, \boldsymbol{F}:=\left(\boldsymbol{f}_{p, q}\right)_{p, q=1 \ldots n}$ und $A:=\left(a_{p, q}\right)_{p, q=1 \ldots n}$ schreiben, können wir die Abbildungen durch folgende Matrizen beschreiben:

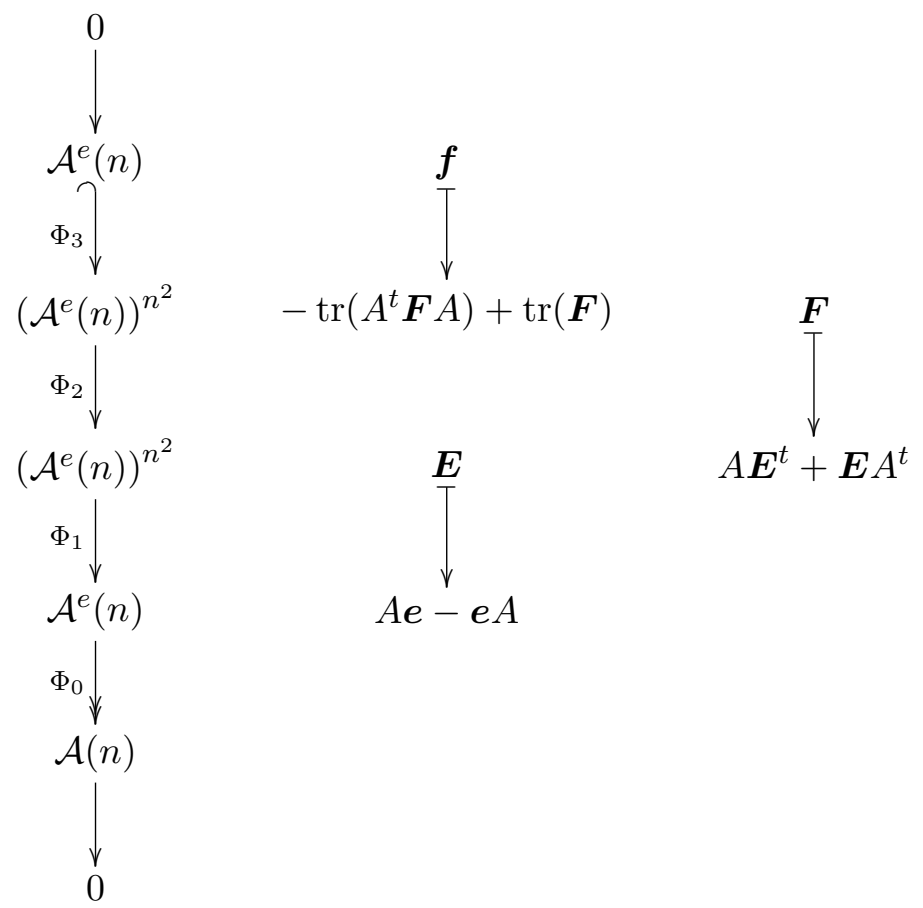

Wir werden für jede Abbildung mit der in 1.3 .3 vorgestellten Methode für den Kern ein Erzeugersystem $S_{\mathrm{Kern}\left(\Phi_{i}\right)}$ berechnen, das mit dem Bild der Erzeuger unter der Abbildung $\Phi_{i+1}$ übereinstimmt. Dazu wählen wir auf $\mathcal{A}^{e}(n)$ die Silbenordnung zu den Trennungsbuchstaben

$$
\begin{array}{ll} 
& \boldsymbol{e}_{n, n}>\cdots>\boldsymbol{e}_{n, 1}>\boldsymbol{e}_{n-1, n}>\cdots>\boldsymbol{e}_{1,1} \\
\text { bzw. } & \boldsymbol{f}_{n, n}>\cdots>\boldsymbol{f}_{n, 1}>\boldsymbol{f}_{n-1, n}>\cdots>\boldsymbol{f}_{1,1} .
\end{array}
$$

Sei $r_{\boldsymbol{A}}:=r_{\mathcal{A}(n)}$ wie in Abschnitt 2.1 definiert. Um ein Erzeugersystem für den $\operatorname{Kern}(\Phi)$ zu erhalten müssen wir ein Erzeugersystem $S=S_{\Phi} \cup S_{e s} \cup S_{\operatorname{Kern}(\Phi)} \cup S_{f s}$ für den Graphen $\Gamma(\Phi)$ angeben, so dass das induzierte Wortersetzungssystem $r_{\boldsymbol{A}, \Gamma(\Phi)}$ schwach vollständig ist (vgl. 1.3.3).

Für jede Abbildung betrachten wir zunächst eine Übersichtstabelle, aus der hervorgeht, welche einzelnen Beweisschritte gemacht werden müssen. Die Tabelle ist wie folgt aufgebaut:

1. Zeile: Die Abbildung in Matrixschreibweise.

2. Zeile: Die Abbildung in Komponentenschreibweise.

3. Zeile: Die Regeln $r_{\Phi}$. Sie erhält man, indem man die Abbildung so umstellt, dass das größte Monom auf der linken Seite steht. Schreibt man sie als Gleichung, so erhält man $S_{\Phi}$.

4. Zeile: Die Regeln $r_{e s}$. Falls $r_{e s}$ nicht benötigt wird, lassen wir die Zeile leer. Schreibt man sie als Gleichung, so erhält man $S_{e s}$. Zusammen mit $r_{\Phi}$ sind es 
die einzigen Regeln, die ein Monom mit einem Erzeuger des Bildmoduls reduzieren, also ist $r_{e}:=r_{\Phi} \cup r_{e s}$. Auf der dahinter angegebenen Seite findet sich ein Beweis, dass $S_{e s} \subset<S_{\Phi}>$.

5. Zeile: Die Regeln $r_{\operatorname{Kern}(\Phi)}$. Schreibt man sie als Gleichung, so erhält man $S_{\operatorname{Kern}(\Phi)}$. Auf der dahinter angegebenen Seite findet sich ein Beweis, dass $S_{\mathrm{Kern}(\Phi)} \subset<$ $S_{\Phi} \cup S_{e s}>$.

6. Zeile: Die Regeln $r_{f s}$. Falls $r_{e s}$ nicht benötigt wird, lassen wir die Zeile leer. Zusammen mit $r_{\text {Kern }(\Phi)}$ sind es die einzigen Regeln die Monome mit einem Erzeuger des Urbildmoduls reduzieren, also ist $r_{f}:=r_{\operatorname{Kern}(\Phi)} \cup r_{f_{s}}$. Nach Satz 1.3.30 und 1.3.33 ist $\left\langle\pi_{1}(x-y) \mid(x, y) \in r_{f}\right\rangle=\operatorname{Kern}(\Phi)$, falls $r_{\boldsymbol{A}} \cup r_{f} \cup r_{e}$ schwach vollständig ist. Schreibt man sie als Gleichung, so erhält man $S_{f s}$. Auf der dahinter angegebenen Seite findet sich ein Beweis, dass $S_{f s} \subset<S_{\Phi} \cup S_{e s}>$ und ein Beweis, dass gilt:

$$
\left\langle\pi_{1}(x-y) \mid(x, y) \in r_{f s}\right\rangle \subset\left\langle\pi_{1}(x-y) \mid(x, y) \in r_{\operatorname{Kern}(\Phi)}\right\rangle .
$$

Also ist $S_{\text {Kern( }(\Phi)}$ ein Erzeugersystem für den Kern.

7. Zeile: Eine Tabelle mit allen minimalen Überschneidungen $r_{A} \cup r_{e} \cup r_{f}$, in denen genau ein Erzeuger des Bildmoduls vorkommt. Um Satz 1.3.33 nutzen zu können müssen alle minimalen Überschneidungen, die höchstens einen Erzeuger des Bildmoduls enthalten, betrachtet werden. Die Überschneidungen, in denen kein Erzeuger vorkommt, wurden jedoch schon in Abschnitt 2.2 überprüft. Ein Eintrag $w$ in der Tabelle ist als Wort einer Überschneidung ( $w$, Zeile, Spalte) zu verstehen.

\section{Bemerkung 3.1.1 (Computerunterstützung)}

Die Regeln $r_{e s}, r_{\text {Kern }(\Phi)}$ und $r_{f s}$ wurden mit Computerunterstützung geraten. Benutzt wurden neben viel selbst geschriebener Software die Programme: Plural [GLS03], GAP [GAP06] und Magma [BCP97]. 


\section{$3.2 \Phi_{1}$}

\subsection{1 Übersichtstabelle für $\Phi_{1}$}

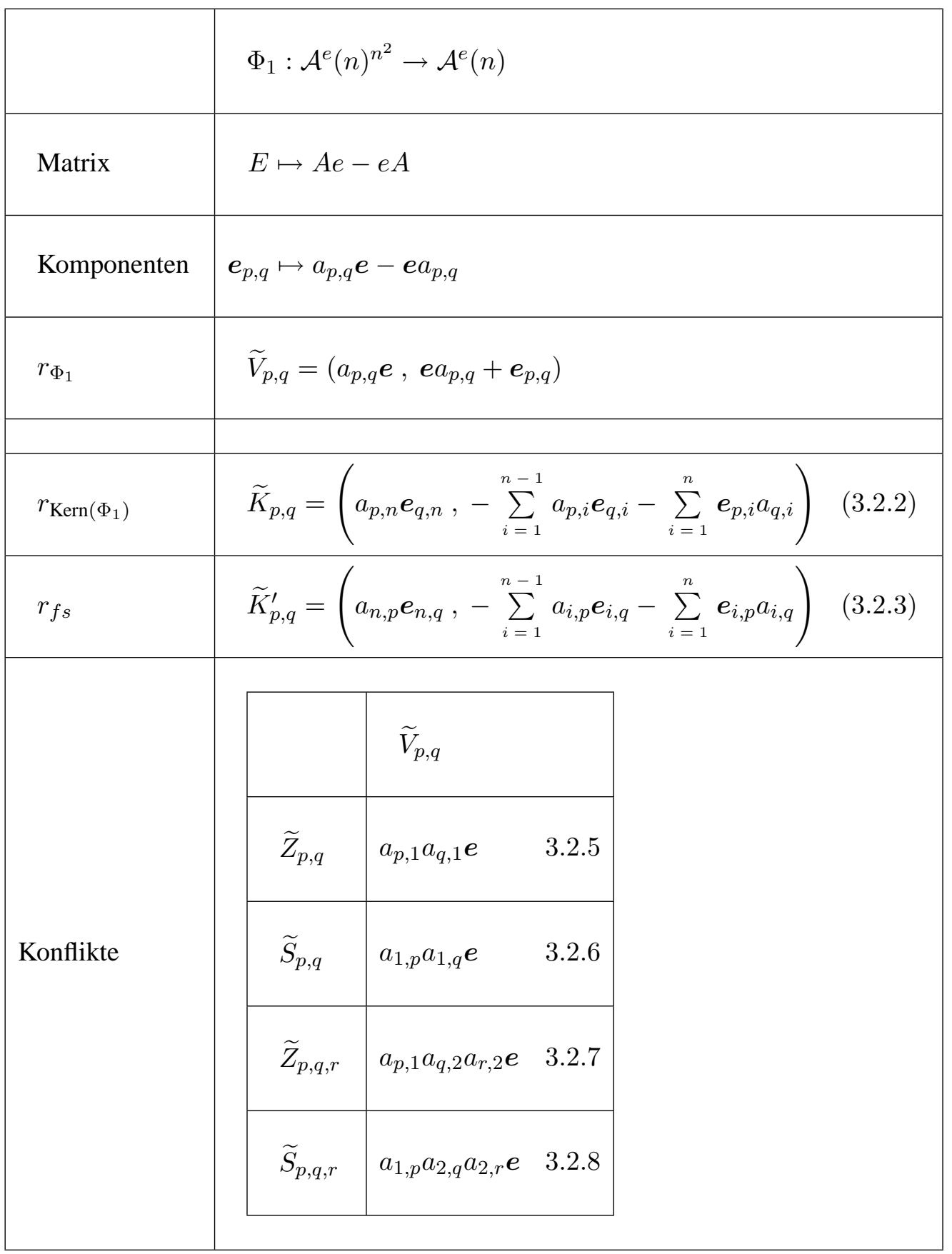




\subsection{2 $r_{\operatorname{Kern}\left(\Phi_{1}\right)}$}

Wir werden zeigen, dass $S_{\operatorname{Kern}\left(\Phi_{1}\right)} \subset\left\langle S_{\Phi_{1}}\right\rangle$. Dazu schreiben wir $S_{\Phi_{1}}$ in Matrizenschreibweise:

$$
\boldsymbol{E}-(A \boldsymbol{e}-\boldsymbol{e} A)
$$

Wenn wir von rechts mit $A^{t}$ multiplizieren, erhalten wir wegen $A A^{t}=\mathrm{id}$ :

$$
\boldsymbol{E} A^{t}-A \boldsymbol{e} A^{t}+\boldsymbol{e} .
$$

Wenn wir zunächst transponieren und dann von links mit $A$ multiplizieren, erhalten wir wegen $A^{t} A=$ id:

$$
A \boldsymbol{E}^{t}-\boldsymbol{e}+A \boldsymbol{e} A^{t} .
$$

Durch Addieren erhalten wir $\boldsymbol{E} A^{t}-A \boldsymbol{E}^{t} \subset\left\langle S_{\Phi_{1}}\right\rangle$.

Da $\boldsymbol{E} A^{t}-A \boldsymbol{E}^{t}$ genau $S_{\text {Kern }\left(\Phi_{1}\right)}$ in Matrixschreibweise ist, folgt die Behauptung.

\subsection{3 $r_{f s}$}

Wir werden zeigen, dass $S_{f_{s}} \subset\left\langle S_{\operatorname{Kern}\left(\Phi_{1}\right)}\right\rangle$. Zusammen mit dem vorhergehenden Abschnitt folgt dann auch $S_{f s} \subset\left\langle S_{\Phi_{1}}\right\rangle$.

Dazu schreiben wir $S_{\mathrm{Kern}\left(\Phi_{1}\right)}$ in Matrizenschreibweise:

$$
\boldsymbol{E} A^{t}-A \boldsymbol{E}^{t}
$$

Wenn wir von links mit $A^{t}$ und von rechts mit $A$ multiplizieren, erhalten wir:

$$
A^{t} \boldsymbol{E}-\boldsymbol{E}^{t} A,
$$

was $S_{f s}$ in Matrizenschreibweise entspricht; also ist $S_{f s} \subset\left\langle S_{\operatorname{Kern}\left(\Phi_{1}\right)}\right\rangle$. 


\subsubsection{Rechenregeln}

Satz 3.2.1 (Rechenregeln)

Es gilt:

$$
\begin{aligned}
& \text { 1. } Z_{p, q} \boldsymbol{e}|\longrightarrow \ldots| \longrightarrow \boldsymbol{e} Z_{p, q}+\boldsymbol{e}_{p, 1} a_{q, 1}+a_{p, 1} e_{q, 1} \\
& \text { 2. } S_{p, q} \boldsymbol{e}|\longrightarrow \ldots| \longrightarrow \boldsymbol{e} S_{p, q}+e_{1, p} a_{1, q}+a_{1, p} e_{1, q} \\
& \text { 3. } Z_{p, q, r} \boldsymbol{e}|\longrightarrow \ldots| \longrightarrow \boldsymbol{e} Z_{p, q, r}+\boldsymbol{e}_{p, 1}\left(Z_{q, r}+a_{q, 2} a_{r, 2}\right)+a_{p, 1} \boldsymbol{e}_{q, 1} a_{r, 1} \\
& +a_{p, 1} \boldsymbol{e}_{q, 2} a_{r, 2}+Z_{p, q} \boldsymbol{e}_{r, 1}+a_{p, 1} a_{q, 2} \boldsymbol{e}_{r, 2} \\
& \text { 4. } S_{p, q, r} \boldsymbol{e}|\longrightarrow \ldots| \longrightarrow \boldsymbol{e} S_{p, q, r}+\boldsymbol{e}_{1, p}\left(S_{q, r}+a_{2, q} a_{2, r}\right)+a_{1, p} \boldsymbol{e}_{1, q} a_{1, r} \\
& +a_{1, p} \boldsymbol{e}_{2, q} a_{2, r}+S_{p, q} \boldsymbol{e}_{1, r}+a_{1, p} a_{2, q} \boldsymbol{e}_{2, r}
\end{aligned}
$$

Beweis: [] Zu 1.:

$$
\begin{aligned}
& Z_{p, q} \boldsymbol{e}=-\sum_{i=2}^{n} a_{p, i} \underline{a_{q, i} \boldsymbol{e}}+\delta_{p, q} \boldsymbol{e} \\
& \stackrel{\widetilde{V}_{q, i}}{\longrightarrow}-\sum_{i=2}^{n}\left(\underline{\left.a_{p, i} \boldsymbol{e} a_{q, i}+a_{p, i} \boldsymbol{e}_{q, i}\right)+\delta_{p, q} \boldsymbol{e}}\right. \\
& \stackrel{\widetilde{V}_{p, i}}{\longrightarrow}-\sum_{i=2}^{n}\left(\boldsymbol{e} a_{p, i} a_{q, i}+\boldsymbol{e}_{p, i} a_{q, i}+a_{p, i} \boldsymbol{e}_{q, i}\right)+\delta_{p, q} \boldsymbol{e} \\
&=\boldsymbol{e} Z_{p, q}-\sum_{i=2}^{n} \boldsymbol{e}_{p, i} a_{q, i}-\sum_{i=2}^{n-1} a_{p, i} \boldsymbol{e}_{q, i}-\underline{a_{p, n} \boldsymbol{e}_{q, n}} \\
& \stackrel{\widetilde{K}_{p, q}}{\longrightarrow} \boldsymbol{e} Z_{p, q}-\sum_{i=2}^{n} \boldsymbol{e}_{p, i} a_{q, i}-\sum_{i=2}^{n-1} a_{p, i} \boldsymbol{e}_{q, i}+\sum_{i=1}^{n-1} a_{p, i} \boldsymbol{e}_{q, i}+\sum_{i=1}^{n} \boldsymbol{e}_{p, i} a_{q, i} \\
&=\boldsymbol{e} Z_{p, q}+\boldsymbol{e}_{p, 1} a_{q, 1}+a_{p, 1} \boldsymbol{e}_{q, 1}
\end{aligned}
$$


$\mathrm{Zu}$ 2.: Diese Rechnung ist nach Vertauschen der Indizes identisch zu der vorhergehenden:

$$
\begin{aligned}
& S_{p, q} \boldsymbol{e}=-\sum_{i=2}^{n} a_{i, p} \underline{a_{i, q} \boldsymbol{e}}+\delta_{p, q} \boldsymbol{e} \\
& \mid \stackrel{\widetilde{V}_{i, q}}{\longrightarrow}-\sum_{i=2}^{n}\left(\underline{a_{i, p} \boldsymbol{e}} a_{i, q}+a_{i, p} \boldsymbol{e}_{i, q}\right)+\delta_{p, q} \boldsymbol{e} \\
& \stackrel{\widetilde{V}_{i, p}}{\longrightarrow}-\sum_{i=2}^{n}\left(\boldsymbol{e} a_{i, p} a_{i, q}+\boldsymbol{e}_{i, p} a_{i, q}+a_{i, p} \boldsymbol{e}_{i, q}\right)+\delta_{p, q} \boldsymbol{e} \\
& \quad=\boldsymbol{e} S_{p, q}-\sum_{i=2}^{n} \boldsymbol{e}_{i, p} a_{i, q}-\sum_{i=2}^{n-1} a_{i, p} \boldsymbol{e}_{i, q}-\underline{a_{n, p} \boldsymbol{e}_{n, q}} \\
& \stackrel{\widetilde{K}_{p, q}^{\prime}}{\longrightarrow} \boldsymbol{e} S_{p, q}-\sum_{i=2}^{n} \boldsymbol{e}_{i, p} a_{i, q}-\sum_{i=2}^{n-1} a_{i, p} \boldsymbol{e}_{i, q}+\sum_{i=1}^{n-1} a_{i, p} \boldsymbol{e}_{i, q}+\sum_{i=1}^{n} \boldsymbol{e}_{i, p} a_{i, q} \\
&=\boldsymbol{e} S_{p, q}+\boldsymbol{e}_{1, p} a_{1, q}+a_{1, p} \boldsymbol{e}_{1, q}
\end{aligned}
$$


$\mathrm{Zu} 3$.:

$$
\begin{aligned}
& Z_{p, q, r} \boldsymbol{e}=-\sum_{i=3}^{n} a_{p, 1} a_{q, i} \underline{a_{r, i} \boldsymbol{e}}+\delta_{q, r} \underline{a_{p, 1} \boldsymbol{e}} \\
& \mid \stackrel{\widetilde{V}_{r, i}, \widetilde{V}_{p, 1}}{\longrightarrow}-\sum_{i=3}^{n} a_{p, 1} \underline{a_{q, i} \boldsymbol{e}} a_{r, i}-\sum_{i=3}^{n} a_{p, 1} a_{q, i} \boldsymbol{e}_{r, i}+\delta_{q, r}\left(\boldsymbol{e} a_{p, 1}+\boldsymbol{e}_{p, 1}\right) \\
& \stackrel{\widetilde{V}_{q, i}}{\longrightarrow}-\sum_{i=3}^{n} \underline{a_{p, 1} \boldsymbol{e}} a_{q, i} a_{r, i}-\sum_{i=3}^{n} a_{p, 1} \boldsymbol{e}_{q, i} a_{r, i} \\
& -\sum_{i=3}^{n-1} a_{p, 1} a_{q, i} \boldsymbol{e}_{r, i}-a_{p, 1} \underline{a_{q, n} \boldsymbol{e}_{r, n}}+\delta_{q, r} \boldsymbol{e} a_{p, 1}+\delta_{q, r} \boldsymbol{e}_{p, 1} \\
& \mid \stackrel{\widetilde{V}_{p, 1}, \widetilde{K}_{q, r}}{\longrightarrow}-\sum_{i=3}^{n} \boldsymbol{e} a_{p, 1} a_{q, i} a_{r, i}-\sum_{i=3}^{n} \boldsymbol{e}_{p, 1} a_{q, i} a_{r, i}-a_{p, 1} \sum_{i=3}^{n} \boldsymbol{e}_{q, i} a_{r, i} \\
& -\sum_{i=3}^{n-1} a_{p, 1} a_{q, i} \boldsymbol{e}_{r, i}+a_{p, 1}\left(\sum_{i=1}^{n-1} a_{q, i} \boldsymbol{e}_{r, i}+\sum_{i=1}^{n} \boldsymbol{e}_{q, i} a_{r, i}\right) \\
& +e a_{p, 1} \delta_{q, r}+e_{p, 1} \delta_{q, r} \\
& =\boldsymbol{e} Z_{p, q, r}+\boldsymbol{e}_{p, 1}\left(Z_{q, r}+a_{q, 2} a_{r, 2}\right)+a_{p, 1}\left(\boldsymbol{e}_{q, 1} a_{r, 1}+\boldsymbol{e}_{q, 2} a_{r, 2}\right) \\
& +a_{p, 1} a_{q, 1} \boldsymbol{e}_{r, 1}+a_{p, 1} a_{q, 2} \boldsymbol{e}_{r, 2} \\
& \stackrel{\widetilde{Z}_{p, q}}{\longrightarrow} e Z_{p, q, r}+\boldsymbol{e}_{p, 1}\left(Z_{q, r}+a_{q, 2} a_{r, 2}\right)+a_{p, 1}\left(\boldsymbol{e}_{q, 1} a_{r, 1}+\boldsymbol{e}_{q, 2} a_{r, 2}\right) \\
& +Z_{p, q} \boldsymbol{e}_{r, 1}+a_{p, 1} a_{q, 2} \boldsymbol{e}_{r, 2}
\end{aligned}
$$


$\mathrm{Zu}$ 4.:

Diese Rechnung ist nach Vertauschen der Indizes identisch zu der vorhergehenden:

$$
\begin{aligned}
& S_{p, q, r} \boldsymbol{e}=-\sum_{i=3}^{n} a_{1, p} a_{i, q} \underline{a_{i, r} \boldsymbol{e}}+\delta_{q, r} \underline{a_{1, p} \boldsymbol{e}} \\
& \mid \stackrel{\widetilde{V}_{i, r}, \widetilde{V}_{1, p}}{\rightarrow}-\sum_{i=3}^{n} a_{1, p} \underline{a_{i, q} \boldsymbol{e}} a_{i, r}-\sum_{i=3}^{n} a_{1, p} a_{i, q} \boldsymbol{e}_{i, r}+\delta_{q, r}\left(\boldsymbol{e} a_{1, p}+\boldsymbol{e}_{1, p}\right) \\
& \stackrel{\widetilde{V}_{i, q}}{\longrightarrow}-\sum_{i=3}^{n} \underline{a_{1, p} \boldsymbol{e}} a_{i, q} a_{i, r}-\sum_{i=3}^{n} a_{1, p} \boldsymbol{e}_{i, q} a_{i, r} \\
& -\sum_{i=3}^{n-1} a_{1, p} a_{i, q} \boldsymbol{e}_{i, r}-a_{1, p} \underline{a_{n, q} \boldsymbol{e}_{n, r}}+\delta_{q, r} \boldsymbol{e} a_{1, p}+\delta_{q, r} \boldsymbol{e}_{1, p} \\
& \stackrel{\widetilde{V}_{1, p}, \widetilde{K}_{q, r}^{\prime}}{\longrightarrow}-\sum_{i=3}^{n} \boldsymbol{e} a_{1, p} a_{i, q} a_{i, r}-\sum_{i=3}^{n} \boldsymbol{e}_{1, p} a_{i, q} a_{i, r}-a_{1, p} \sum_{i=3}^{n} \boldsymbol{e}_{i, q} a_{i, r} \\
& -\sum_{i=3}^{n-1} a_{1, p} a_{i, q} \boldsymbol{e}_{i, r}+a_{1, p}\left(\sum_{i=1}^{n-1} a_{i, q} \boldsymbol{e}_{i, r}+\sum_{i=1}^{n} \boldsymbol{e}_{i, q} a_{i, r}\right) \\
& +\boldsymbol{e} a_{1, p} \delta_{q, r}+\boldsymbol{e}_{1, p} \delta_{q, r} \\
& =\boldsymbol{e} S_{p, q, r}+\boldsymbol{e}_{1, p}\left(S_{q, r}+a_{2, q} a_{2, r}\right)+a_{1, p}\left(\boldsymbol{e}_{1, q} a_{1, r}+\boldsymbol{e}_{2, q} a_{2, r}\right) \\
& +\underline{a_{1, p} a_{1, q}} \boldsymbol{e}_{1, r}+a_{1, p} a_{2, q} \boldsymbol{e}_{2, r} \\
& \stackrel{\widetilde{Z}_{p, q}}{\longrightarrow} e S_{p, q, r}+e_{1, p}\left(S_{q, r}+a_{2, q} a_{2, r}\right)+a_{1, p}\left(e_{1, q} a_{1, r}+e_{2, q} a_{2, r}\right) \\
& +S_{p, q} \boldsymbol{e}_{1, r}+a_{1, p} a_{2, q} \boldsymbol{e}_{2, r}
\end{aligned}
$$




\subsubsection{Reduktionswege für $a_{p, 1} a_{q, 1} \boldsymbol{e}$}

Beginnend mit $\left(a_{p, 1} a_{q, 1} e, Z_{p, q} e\right)$ :

$$
\begin{aligned}
\underline{a_{p, 1} a_{q, 1}} \boldsymbol{e} \mid \stackrel{\widetilde{Z}_{p, q}}{\longrightarrow} Z_{p, q} \boldsymbol{~} \\
\mid \stackrel{3.2 .4}{\longrightarrow} \boldsymbol{e} Z_{p, q}+\boldsymbol{e}_{p, 1} a_{q, 1}+a_{p, 1} \boldsymbol{e}_{q, 1}
\end{aligned}
$$

Beginnend mit $\left(a_{p, 1} a_{q, 1} \boldsymbol{e}, a_{p, 1} V_{q, 1}\right)$ :

$$
\begin{aligned}
& a_{p, 1} \underline{a_{q, 1} \boldsymbol{e}} \mid \stackrel{\widetilde{V}_{q, 1}}{\longrightarrow} \underline{a_{p, 1} \boldsymbol{e} a_{q, 1}}+a_{p, 1} \boldsymbol{e}_{q, 1} \\
& \mid \stackrel{\widetilde{V}_{p, 1}}{\longrightarrow} \boldsymbol{e} \underline{a_{p, 1} a_{q, 1}}+\boldsymbol{e}_{p, 1} a_{q, 1}+a_{p, 1} \boldsymbol{e}_{q, 1} \\
& \mid \stackrel{\widetilde{Z}_{p, q}}{\longrightarrow} \boldsymbol{e} Z_{p, q}+\boldsymbol{e}_{p, 1} a_{q, 1}+a_{p, 1} \boldsymbol{e}_{q, 1}
\end{aligned}
$$

Bemerkung 3.2.2 (Symmetrie)

Diese Rechnungen sind nach Vertauschen der Indizes identisch mit denen in 3.2.6. 


\subsubsection{Reduktionswege für $a_{1, p} a_{1, q} e$}

Beginnend mit $\left(a_{1, p} a_{1, q} \boldsymbol{e}, S_{p, q} \boldsymbol{e}\right)$ :

$$
\begin{aligned}
& \underline{a_{1, p} a_{1, q}} \boldsymbol{e} \mid \stackrel{\widetilde{S}_{p, q}}{\longrightarrow} \underline{S_{p, q} e} \\
& \stackrel{3.2 .4}{\longrightarrow} e S_{p, q}+e_{1, p} a_{1, q}+a_{1, p} \boldsymbol{e}_{1, q}
\end{aligned}
$$

Beginnend mit $\left(a_{1, p} a_{1, q} \boldsymbol{e}, a_{1, p} V_{1, q}\right)$ :

$$
\begin{aligned}
& a_{1, p} \underline{a_{1, q} \boldsymbol{e}} \mid \stackrel{\widetilde{V}_{1, q}}{\longrightarrow} \underline{a_{1, p} \boldsymbol{e} a_{1, q}+a_{1, p} \boldsymbol{e}_{1, q}} \\
& \mid \stackrel{\widetilde{V}_{1, p}}{\longrightarrow} \boldsymbol{e} \underline{a_{1, p} a_{1, q}}+\boldsymbol{e}_{1, p} a_{1, q}+a_{1, p} \boldsymbol{e}_{1, q} \\
& \stackrel{\widetilde{S}_{p, q}}{\longrightarrow} \boldsymbol{e} S_{p, q}+\boldsymbol{e}_{1, p} a_{1, q}+a_{1, p} \boldsymbol{e}_{1, q}
\end{aligned}
$$

Bemerkung 3.2.3 (Symmetrie)

Diese Rechnungen sind nach Vertauschen der Indizes identisch mit denen in 3.2.5. 


\subsubsection{Reduktionswege für $a_{p, 1} a_{q, 2} a_{r, 2} \boldsymbol{e}$}

Beginnend mit $\left(a_{p, 1} a_{q, 2} a_{r, 2} \boldsymbol{e},\left(Z_{p, q, r}-Z_{p, q} a_{r, 1}\right) \boldsymbol{e}\right)$ :

$$
\begin{aligned}
& \underline{a_{p, 1} a_{q, 2} a_{r, 2}} \boldsymbol{e} \\
& \stackrel{\widetilde{Z}_{p, q, r}}{\longrightarrow} \underline{Z_{p, q, r} \boldsymbol{e}}-Z_{p, q} \underline{a_{r, 1} \boldsymbol{e}} \\
& 3.2 .4 . \widetilde{V}_{r, 1} \\
& \boldsymbol{e} Z_{p, q, r}+\boldsymbol{e}_{p, 1}\left(Z_{q, r}+a_{q, 2} a_{r, 2}\right)+a_{p, 1}\left(\boldsymbol{e}_{q, 1} a_{r, 1}+\boldsymbol{e}_{q, 2} a_{r, 2}\right) \\
& +Z_{p, q} \boldsymbol{e}_{r, 1}+a_{p, 1} a_{q, 2} \boldsymbol{e}_{r, 2}-\underline{Z_{p, q}} \boldsymbol{e} a_{r, 1}-Z_{p, q} \boldsymbol{e}_{r, 1} \\
& 3.2 .4 \\
& \boldsymbol{e} Z_{p, q, r}+\boldsymbol{e}_{p, 1}\left(Z_{q, r}+a_{q, 2} a_{r, 2}\right)+a_{p, 1}\left(\boldsymbol{e}_{q, 1} a_{r, 1}+\boldsymbol{e}_{q, 2} a_{r, 2}\right) \\
& +a_{p, 1} a_{q, 2} \boldsymbol{e}_{r, 2}-\left(\boldsymbol{e} Z_{p, q}+\boldsymbol{e}_{p, 1} a_{q, 1}+a_{p, 1} e_{q, 1}\right) a_{r, 1} \\
& =-\boldsymbol{e} Z_{p, q, r}+\boldsymbol{e}_{p, 1}\left(Z_{q, r}-\underline{a_{q, 1} a_{r, 1}}+a_{q, 2} a_{r, 2}\right)+a_{p, 1} \boldsymbol{e}_{q, 2} a_{r, 2} \\
& +a_{p, 1} a_{q, 2} \boldsymbol{e}_{r, 2}-\boldsymbol{e} Z_{p, q} a_{r, 1} \\
& \stackrel{\widetilde{Z}_{q, r}}{\longrightarrow} e Z_{p, q, r}+e_{p, 1}\left(Z_{q, r}-Z_{q, r}+a_{q, 2} a_{r, 2}\right)+a_{p, 1} e_{q, 2} a_{r, 2} \\
& +a_{p, 1} a_{q, 2} \boldsymbol{e}_{r, 2}-\boldsymbol{e} Z_{p, q} a_{r, 1}
\end{aligned}
$$

Beginnend mit $\left(a_{p, 1} a_{q, 2} a_{r, 2} e, a_{p, 1} a_{q, 2} V_{r, 2}\right)$ :

$$
\begin{aligned}
& \mid \stackrel{\widetilde{V}_{r, 2}}{\longrightarrow} a_{p, 1} \underline{a_{q, 2} \boldsymbol{e} a_{r, 2}}+a_{p, 1} a_{q, 2} \boldsymbol{e}_{r, 2} \\
& \stackrel{\widetilde{V}_{q, 2}}{\longrightarrow} \underline{a_{p, 1} \boldsymbol{e} a_{q, 2} a_{r, 2}+a_{p, 1} \boldsymbol{e}_{q, 2} a_{r, 2}+a_{p, 1} a_{q, 2} \boldsymbol{e}_{r, 2}} \\
& \mid \stackrel{\widetilde{V}_{p, 1}}{\longrightarrow} e \underline{\boldsymbol{e}} \underline{a_{p, 1} a_{q, 2} a_{r, 2}}+\boldsymbol{e}_{p, 1} a_{q, 2} a_{r, 2}+a_{p, 1} \boldsymbol{e}_{q, 2} a_{r, 2}+a_{p, 1} a_{q, 2} \boldsymbol{e}_{r, 2} \\
& \stackrel{\widetilde{Z}_{p, q, r}}{\longrightarrow} \boldsymbol{e} Z_{p, q, r}-\boldsymbol{e} Z_{p, q} a_{r, 1}+\boldsymbol{e}_{p, 1} a_{q, 2} a_{r, 2}+a_{p, 1} \boldsymbol{e}_{q, 2} a_{r, 2}+a_{p, 1} a_{q, 2} \boldsymbol{e}_{r, 2}
\end{aligned}
$$

\section{Bemerkung 3.2.4 (Symmetrie)}

Diese Rechnungen sind nach Vertauschen der Indizes identisch mit denen in 3.2.8 


\subsubsection{Reduktionswege für $a_{1, p} a_{2, q} a_{2, r} \boldsymbol{e}$}

Beginnend mit $\left(a_{1, p} a_{2, q} a_{2, r} \boldsymbol{e},\left(S_{p, q, r}-S_{p, q} a_{1, r}\right) \boldsymbol{e}\right)$ :

$$
\begin{aligned}
& a_{1, p} a_{2, q} a_{2, r} \boldsymbol{e} \\
& \stackrel{\widetilde{S}_{p, q, r}}{\longrightarrow} \underline{S_{p, q, r} \boldsymbol{e}}-S_{p, q} \underline{a_{1, r} \boldsymbol{e}} \\
& \text { 3.2.4. } \widetilde{V}_{1, r} \\
& \boldsymbol{e} S_{p, q, r}+\boldsymbol{e}_{1, p}\left(S_{q, r}+a_{2, q} a_{2, r}\right)+a_{1, p}\left(\boldsymbol{e}_{1, q} a_{1, r}+\boldsymbol{e}_{2, q} a_{2, r}\right) \\
& +S_{p, q} \boldsymbol{e}_{1, r}+a_{1, p} a_{2, q} \boldsymbol{e}_{2, r}-S_{p, q} \boldsymbol{e} a_{1, r}-S_{p, q} \boldsymbol{e}_{1, r} \\
& \stackrel{3.2 .4}{\longrightarrow} \boldsymbol{e} S_{p, q, r}+\boldsymbol{e}_{1, p}\left(S_{q, r}+a_{2, q} a_{2, r}\right)+a_{1, p}\left(\boldsymbol{e}_{1, q} a_{1, r}+\boldsymbol{e}_{2, q} a_{2, r}\right) \\
& +a_{1, p} a_{2, q} e_{2, r}-\left(e S_{p, q}+\boldsymbol{e}_{1, p} a_{1, q}+a_{1, p} e_{1, q}\right) a_{1, r} \\
& =-\boldsymbol{e} S_{p, q, r}+\boldsymbol{e}_{1, p}\left(S_{q, r}-\underline{a_{1, q} a_{1, r}}+a_{2, q} a_{2, r}\right)+a_{1, p} \boldsymbol{e}_{2, q} a_{2, r} \\
& +a_{1, p} a_{2, q} \boldsymbol{e}_{2, r}-\boldsymbol{e} S_{p, q} a_{1, r} \\
& \mid \stackrel{\widetilde{S}_{q, r}}{\longrightarrow} e S_{p, q, r}+e_{1, p}\left(S_{q, r}-S_{q, r}+a_{2, q} a_{2, r}\right)+a_{1, p} \boldsymbol{e}_{2, q} a_{2, r} \\
& +a_{1, p} a_{2, q} \boldsymbol{e}_{2, r}-\boldsymbol{e} S_{p, q} a_{1, r}
\end{aligned}
$$

Beginnend mit $\left(a_{1, p} a_{2, q} a_{2, r} \boldsymbol{e}, a_{1, p} a_{2, q} V_{2, r}\right)$ :

$$
\begin{aligned}
& a_{1, p} a_{2, q} a_{2, r} \boldsymbol{e} \\
& \widetilde{V}_{2, r}
\end{aligned}
$$

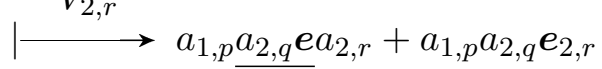

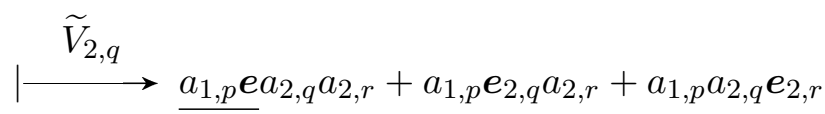

$$
\begin{aligned}
& \mid \stackrel{\widetilde{V}_{1, p}}{\longrightarrow} \boldsymbol{e a}^{a_{1, p} a_{2, q} a_{2, r}}+\boldsymbol{e}_{1, p} a_{2, q} a_{2, r}+a_{1, p} \boldsymbol{e}_{2, q} a_{2, r}+a_{1, p} a_{2, q} \boldsymbol{e}_{2, r} \\
& \stackrel{\widetilde{S}_{p, q, r}}{\longrightarrow} e S_{p, q, r}-e S_{p, q} a_{1, r}+e_{1, p} a_{2, q} a_{2, r}+a_{1, p} e_{2, q} a_{2, r}+a_{1, p} a_{2, q} e_{2, r}
\end{aligned}
$$

\section{Bemerkung 3.2.5 (Symmetrie)}

Diese Rechnungen sind nach Vertauschen der Indizes identisch mit denen in 3.2.7 


\section{3 $\Phi_{2}$}

\subsection{1 Übersichtstabelle für $\Phi_{2}$}

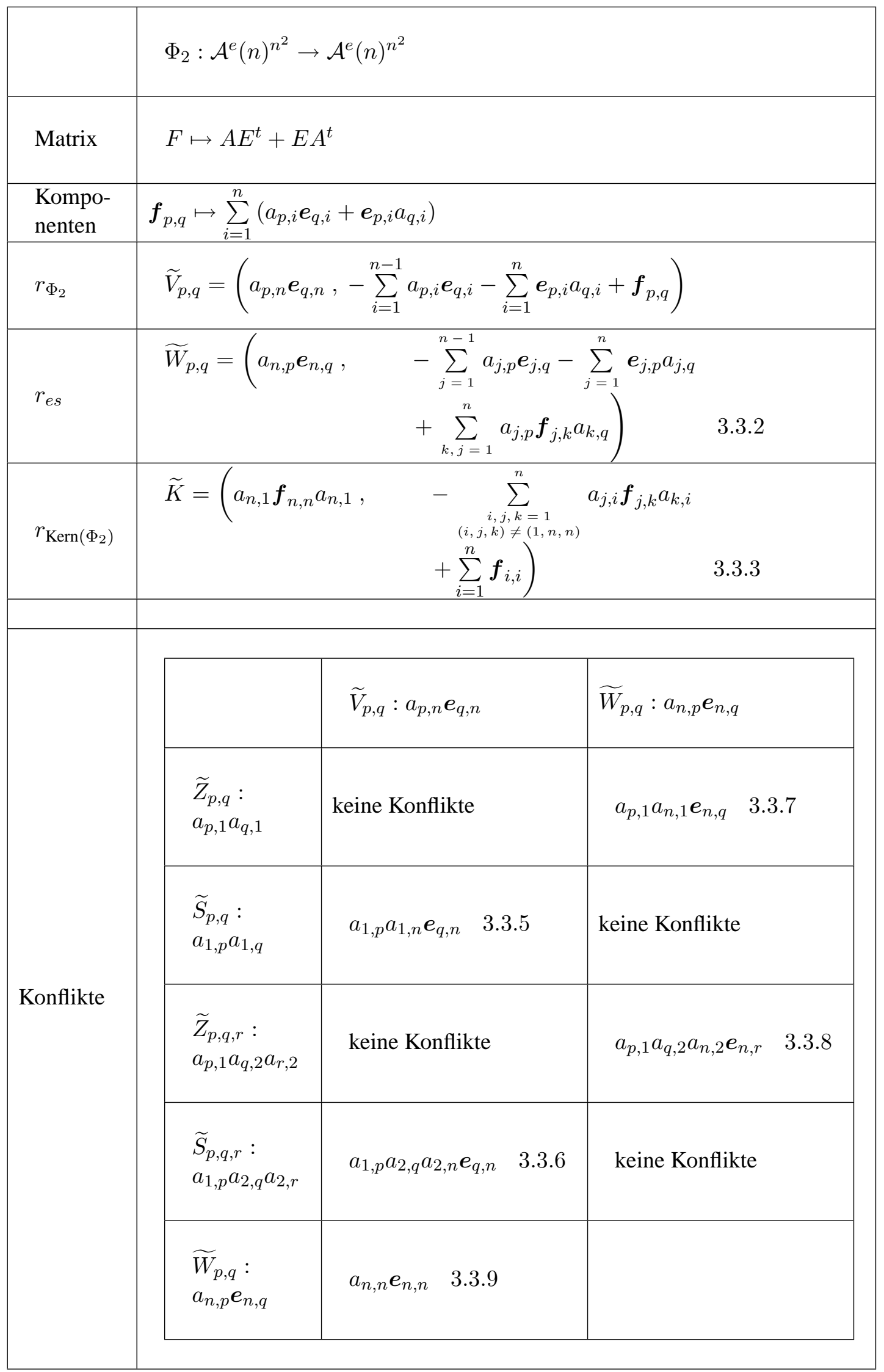




\subsection{2 $r_{e s}$}

Wir werden zeigen, dass $S_{e s} \subset\left\langle S_{\Phi_{2}}\right\rangle$. Dazu schreiben wir $S_{\Phi_{2}}$ in Matrizenschreibweise:

$$
\boldsymbol{F}-\left(A \boldsymbol{E}^{t}+\boldsymbol{E} A^{t}\right) .
$$

Wenn wir von links mit $A^{t}$ und von rechts mit $A$ multiplizieren, erhalten wir, da $A^{t} A=$ id:

$$
A^{t} \boldsymbol{F} A-\boldsymbol{E}^{t} A-A^{t} \boldsymbol{E} .
$$

Also ist $A^{t} \boldsymbol{F} A-\boldsymbol{E}^{t} A-A^{t} \boldsymbol{E} \subset\left\langle S_{\Phi_{2}}\right\rangle$.

Da $A^{t} \boldsymbol{F} A-\boldsymbol{E}^{t} A-A^{t} \boldsymbol{E}$ gerade $S_{e s}$ in Matrixschreibweise ist, folgt die Behauptung.

\subsection{3 $r_{\operatorname{Kern}\left(\Phi_{2}\right)}$}

Wir werden zeigen, dass $S_{\mathrm{Kern}\left(\Phi_{2}\right)} \subset\left\langle S_{\Phi_{2}}\right\rangle$. Dazu schreiben wir $S_{\Phi_{2}}$ in Matrizenschreibweise:

$$
\boldsymbol{F}-\left(A \boldsymbol{E}^{t}+\boldsymbol{E} A^{t}\right) .
$$

Wenn wir die Spur bilden, erhalten wir:

$$
\operatorname{tr}(\boldsymbol{F})-\operatorname{tr}\left(A \boldsymbol{E}^{t}\right)-\operatorname{tr}\left(\boldsymbol{E} A^{t}\right) .
$$

Also ist $\operatorname{tr}(\boldsymbol{F})-\operatorname{tr}\left(A \boldsymbol{E}^{t}\right)-\operatorname{tr}\left(\boldsymbol{E} A^{t}\right) \subset\left\langle S_{\Phi_{2}}\right\rangle$.

Da $S_{\text {Kern }\left(\Phi_{2}\right)}$ in Matizenschreibweise gerade

$$
\operatorname{tr}(\boldsymbol{F})-\operatorname{tr}\left(A^{t} F A\right)
$$

ist, folgt die Behauptung mit folgender Gleichung:

$$
\begin{aligned}
\operatorname{tr}\left(A E^{t}+E A^{t}\right) & =\sum_{i=1}^{n}\left(\left(A E^{t}\right)_{i, i}+\left(E A^{t}\right)_{i, i}\right) \\
& =\sum_{i=1}^{n} \sum_{j=1}^{n}\left(a_{i, j} e_{i, j}+e_{i, j} a_{i, j}\right) \\
& =\sum_{i=j}^{n}\left(\left(E^{t} A\right)_{j, j}+\left(A^{t} E\right)_{j, j}\right) \\
& =\operatorname{tr}\left(A^{t} A E^{t} A+A^{t} E A^{t} A\right)=\operatorname{tr}\left(A^{t} F A\right) .
\end{aligned}
$$




\subsubsection{Rechenregeln}

Satz 3.3.1 (Rechenregeln)

Sei $m \in\{1 \ldots n\}$, dann gilt: 1. :

$$
\sum_{i=1}^{n} a_{p, i} a_{q, i} \mapsto \delta_{p, q}
$$

und $2 .:$

$$
\begin{aligned}
& \sum_{j=m}^{n} a_{j, p} \underline{a_{j, n} \boldsymbol{e}_{q, n}} \mapsto \ldots \mapsto \\
& -\sum_{j=m}^{n} \sum_{i=1}^{n-1} a_{j, p} a_{j, i} \boldsymbol{e}_{q, i}+\sum_{j=1}^{m-1} \sum_{i=1}^{n} a_{j, p} \boldsymbol{e}_{j, i} a_{q, i}+\boldsymbol{e}_{q, p} \\
& -\sum_{j=1}^{m-1} a_{j, p} \boldsymbol{f}_{j, q}
\end{aligned}
$$

und 3.:

$$
\begin{aligned}
& \sum_{i=m}^{n} a_{p, i} a_{n, i} \boldsymbol{e}_{n, q} \mapsto \ldots \mapsto \\
& -\sum_{i=m}^{n} \sum_{j=1}^{n-1} a_{p, i} a_{j, i} \boldsymbol{e}_{j, q}+\sum_{i=1}^{m-1} \sum_{j=1}^{n} a_{p, i} \boldsymbol{e}_{j, i} a_{j, q}+\boldsymbol{e}_{p, q} \\
& -\sum_{j=1}^{n} \boldsymbol{f}_{p, j} a_{j, q}+\sum_{i=m}^{n} \sum_{k, j=1}^{n} a_{p, i} a_{j, i} \boldsymbol{f}_{j, k} a_{k, q}
\end{aligned}
$$

Beweis: $\mathrm{Zu} \mathrm{1.:}$

$$
\begin{aligned}
\sum_{i=1}^{n} a_{p, i} a_{q, i} & =\underline{a_{p, 1} a_{q, 1}}+\sum_{i=2}^{n} a_{p, i} a_{q, i} \\
\mid \stackrel{\widetilde{Z}_{p, q}}{\longrightarrow} & -\sum_{i=2}^{n} a_{p, i} a_{q, i}+\delta_{p, q}+\sum_{i=2}^{n} a_{p, i} a_{q, i} \\
& =\delta_{p, q}
\end{aligned}
$$


$\mathrm{Zu} 2$.:

$$
\begin{aligned}
& \sum_{j=m}^{n} a_{j, p} \underline{a_{j, n} \boldsymbol{e}_{q, n}} \\
\stackrel{\widetilde{V}_{j, q}}{\longrightarrow} & \sum_{j=m}^{n} a_{j, p}\left(-\sum_{i=1}^{n-1} a_{j, i} \boldsymbol{e}_{q, i}-\sum_{i=1}^{n} \boldsymbol{e}_{j, i} a_{q, i}+\boldsymbol{f}_{j, q}\right) \\
= & -\sum_{j=m}^{n} \sum_{i=1}^{n-1} a_{j, p} a_{j, i} \boldsymbol{e}_{q, i}+\sum_{j=m}^{n} a_{j, p} \boldsymbol{f}_{j, q} \\
& -\sum_{i, j=1}^{n-1} a_{j, p} \boldsymbol{e}_{j, i} a_{q, i}-\sum_{j=1}^{n-1} a_{j, p} \boldsymbol{e}_{j, n} a_{q, n} \\
& -\sum_{i=1}^{n-1} \underline{a}_{n, p} \boldsymbol{e}_{n, i} a_{q, i}+\sum_{j=1}^{m-1} \sum_{i=1}^{n} a_{j, p} \boldsymbol{e}_{j, i} a_{q, i}-\underline{a_{n, p} \boldsymbol{e}_{n, n}} a_{q, n}
\end{aligned}
$$

$$
\widetilde{W 2}_{p, i}, \widetilde{W 2}{ }_{p, n}
$$$$
-\sum_{j=m}^{n} \sum_{i=1}^{n-1} a_{j, p} a_{j, i} \boldsymbol{e}_{q, i}+\sum_{j=m}^{n} a_{j, p} \boldsymbol{f}_{j, q}
$$$$
-\sum_{i, j=1}^{n-1} a_{j, p} \boldsymbol{e}_{j, i} a_{q, i}-\sum_{j=1}^{n-1} a_{j, p} \boldsymbol{e}_{j, n} a_{q, n}
$$$$
-\sum_{i=1}^{n-1}\left(-\sum_{j=1}^{n-1} a_{j, p} \boldsymbol{e}_{j, i}-\sum_{j=1}^{n} \boldsymbol{e}_{j, p} a_{j, i}+\sum_{k, j=1}^{n} a_{j, p} \boldsymbol{f}_{j, k} a_{k, i}\right) a_{q, i}
$$$$
+\sum_{j=1}^{m-1} \sum_{i=1}^{n} a_{j, p} \boldsymbol{e}_{j, i} a_{q, i}
$$$$
-\left(-\sum_{j=1}^{n-1} a_{j, p} \boldsymbol{e}_{j, n}-\sum_{j=1}^{n} \boldsymbol{e}_{j, p} a_{j, n}+\sum_{k, j=1}^{n} a_{j, p} \boldsymbol{f}_{j, k} a_{k, n}\right) a_{q, n}
$$$$
=-\sum_{j=m}^{n} \sum_{i=1}^{n-1} a_{j, p} a_{j, i} \boldsymbol{e}_{q, i}+\sum_{j=1}^{m-1} \sum_{i=1}^{n} a_{j, p} \boldsymbol{e}_{j, i} a_{q, i}
$$$$
+\sum_{i, j=1}^{n} \boldsymbol{e}_{j, p} \underline{a_{j, i} a_{q, i}}+\sum_{j=m}^{n} a_{j, p} \boldsymbol{f}_{j, q}-\sum_{i, k, j=1}^{n} a_{j, p} \boldsymbol{f}_{j, k} \underline{a_{k, i} a_{q, i}}
$$ 


$$
\begin{aligned}
\stackrel{3.3 .4}{\longrightarrow} & -\sum_{j=m}^{n} \sum_{i=1}^{n-1} a_{j, p} a_{j, i} \boldsymbol{e}_{q, i}+\sum_{j=1}^{m-1} \sum_{i=1}^{n} a_{j, p} \boldsymbol{e}_{j, i} a_{q, i}+\sum_{j=1}^{n} \boldsymbol{e}_{j, p} \delta_{j, q} \\
& +\sum_{j=m}^{n} a_{j, p} \boldsymbol{f}_{j, q}-\sum_{k, j=1}^{n} a_{j, p} \boldsymbol{f}_{j, k} \delta_{k, q} \\
= & -\sum_{j=m}^{n} \sum_{i=1}^{n-1} a_{j, p} a_{j, i} \boldsymbol{e}_{q, i}+\sum_{j=1}^{m-1} \sum_{i=1}^{n} a_{j, p} \boldsymbol{e}_{j, i} a_{q, i} \\
& +\boldsymbol{e}_{q, p}-\sum_{j=1}^{m-1} a_{j, p} \boldsymbol{f}_{j, q}
\end{aligned}
$$

zu 3.:

$$
\begin{aligned}
& \sum_{i=m}^{n} a_{p, i} \underline{a_{n, i} \boldsymbol{e}_{n, q}} \\
\mid \widetilde{W_{i, q}} & \sum_{i=m}^{n} a_{p, i}\left(-\sum_{j=1}^{n-1} a_{j, i} \boldsymbol{e}_{j, q}-\sum_{j=1}^{n} \boldsymbol{e}_{j, i} a_{j, q}+\sum_{k, j=1}^{n} a_{j, i} \boldsymbol{f}_{j, k} a_{k, q}\right) \\
= & -\sum_{i=m}^{n} \sum_{j=1}^{n-1} a_{p, i} a_{j, i} \boldsymbol{e}_{j, q}-\sum_{i=m}^{n-1} \sum_{j=1}^{n} a_{p, i} \boldsymbol{e}_{j, i} a_{j, q} \\
\widetilde{V_{p, j}} & -\sum_{j=1}^{n} \frac{a_{p, n} \boldsymbol{e}_{j, n} a_{j, q}+\sum_{i=m}^{n} \sum_{k, j=1}^{n} a_{p, i} a_{j, i} \boldsymbol{f}_{j, k} a_{k, q}}{\longrightarrow}-\sum_{i=m}^{n} \sum_{j=1}^{n-1} a_{p, i} a_{j, i} \boldsymbol{e}_{j, q}-\sum_{i=m}^{n-1} \sum_{j=1}^{n} a_{p, i} \boldsymbol{e}_{j, i} a_{j, q} \\
& -\sum_{j=1}^{n}\left(-\sum_{i=1}^{n-1} a_{p, i} \boldsymbol{e}_{j, i}-\sum_{i=1}^{n} \boldsymbol{e}_{p, i} a_{j, i}+\boldsymbol{f}_{p, j}\right) a_{j, q} \\
& +\sum_{i=m}^{n} \sum_{k, j=1}^{n} a_{p, i} a_{j, i} \boldsymbol{f}_{j, k} a_{k, q}
\end{aligned}
$$




$$
\begin{aligned}
= & -\sum_{i=m}^{n} \sum_{j=1}^{n-1} a_{p, i} a_{j, i} \boldsymbol{e}_{j, q}+\sum_{i=1}^{m-1} \sum_{j=1}^{n} a_{p, i} \boldsymbol{e}_{j, i} a_{j, q}+\sum_{i=1}^{n} \boldsymbol{e}_{p, i} \sum_{j=1}^{n} a_{j, i} a_{j, q} \\
& -\sum_{j=1}^{n} \boldsymbol{f}_{p, j} a_{j, q}+\sum_{i=m}^{n} \sum_{k, j=1}^{n} a_{p, i} a_{j, i} \boldsymbol{f}_{j, k} a_{k, q} \\
\underline{\underline{3.3 .4} \longrightarrow}- & \sum_{i=m}^{n} \sum_{j=1}^{n-1} a_{p, i} a_{j, i} \boldsymbol{e}_{j, q}+\sum_{i=1}^{m-1} \sum_{j=1}^{n} a_{p, i} \boldsymbol{e}_{j, i} a_{j, q}+\sum_{i=1}^{n} \boldsymbol{e}_{p, i} \delta_{i, q} \\
& -\sum_{j=1}^{n} \boldsymbol{f}_{p, j} a_{j, q}+\sum_{i=m}^{n} \sum_{k, j=1}^{n} a_{p, i} a_{j, i} \boldsymbol{f}_{j, k} a_{k, q} \\
= & -\sum_{i=m}^{n} \sum_{j=1}^{n-1} a_{p, i} a_{j, i} \boldsymbol{e}_{j, q}+\sum_{i=1}^{m-1} \sum_{j=1}^{n} a_{p, i} \boldsymbol{e}_{j, i} a_{j, q}+\boldsymbol{e}_{p, q} \\
& -\sum_{j=1}^{n} \boldsymbol{f}_{p, j} a_{j, q}+\sum_{i=m}^{n} \sum_{k, j=1}^{n} a_{p, i} a_{j, i} \boldsymbol{f}_{j, k} a_{k, q}
\end{aligned}
$$


3.3.5 Reduktionswege für $a_{1, p} a_{1, n} e_{q, n}$

Beginnend mit $\left(a_{1, p} a_{1, n} \boldsymbol{e}_{q, n}, S_{p, n} \boldsymbol{e}_{q, n}\right)$ :

$$
\begin{aligned}
\stackrel{\widetilde{S}_{p, n}}{\longrightarrow} & -\sum_{j=2}^{n} a_{j, p} a_{j, n} \boldsymbol{e}_{q, n}+\delta_{p, n} \boldsymbol{e}_{q, n} \\
\stackrel{\text { 3.3.4 }}{\longrightarrow} & \sum_{j=2}^{n} \sum_{i=1}^{n-1} a_{j, p} a_{j, i} \boldsymbol{e}_{q, i}-\sum_{i=1}^{n} a_{1, p} \boldsymbol{e}_{1, i} a_{q, i}-\boldsymbol{e}_{q, p} \\
& +a_{1, p} \boldsymbol{f}_{1, q}+\delta_{p, n} \boldsymbol{e}_{q, n}
\end{aligned}
$$

Beginnend mit $\left(a_{1, p} a_{1, n} \boldsymbol{e}_{q, n}, a_{1, p} V_{1, q}\right)$ :

$$
\begin{aligned}
\mid \stackrel{\widetilde{V}_{1, q}}{\longrightarrow} & -\sum_{i=1}^{a_{1, p}} \underline{a_{1, n} \boldsymbol{e}_{q, n}} \underline{a_{1, p} a_{1, i}} \boldsymbol{e}_{q, i}-\sum_{i=1}^{n} a_{1, p} \boldsymbol{e}_{1, i} a_{q, i}+a_{1, p} \boldsymbol{f}_{1, q} \\
\mid \stackrel{\widetilde{S}_{p, i}}{\longrightarrow} & -\sum_{i=1}^{n-1}\left(-\sum_{j=2}^{n} a_{j, p} a_{j, i}+\delta_{p, i}\right) \boldsymbol{e}_{q, i}-\sum_{i=1}^{n} a_{1, p} \boldsymbol{e}_{1, i} a_{q, i}+a_{1, p} \boldsymbol{f}_{1, q} \\
= & \sum_{i=1}^{n-1} \sum_{j=2}^{n} a_{j, p} a_{j, i} \boldsymbol{e}_{q, i}-\left(1-\delta_{p, n}\right) \boldsymbol{e}_{q, p}-\sum_{i=1}^{n} a_{1, p} \boldsymbol{e}_{1, i} a_{q, i}+a_{1, p} \boldsymbol{f}_{1, q}
\end{aligned}
$$




\subsubsection{Reduktionswege für $a_{1, p} a_{2, q} a_{2, n} \boldsymbol{e}_{r, n}$}

Beginnend mit $\left(a_{1, p} a_{2, q} a_{2, n} \boldsymbol{e}_{r, n},\left(S_{p, q, n}-S_{p, q} a_{1, n}\right) \boldsymbol{e}_{r, n}\right)$ :

$$
\begin{aligned}
\stackrel{\widetilde{S}_{p, q, n}}{\longrightarrow}-a_{1, p} \sum_{j=3}^{n} a_{j, q} a_{j, n} \boldsymbol{e}_{r, n}+a_{1, p} \delta_{q, n} \boldsymbol{e}_{r, n}+\left(\sum_{j=2}^{n} a_{j, p} a_{j, q}-\delta_{p, q}\right) \underline{a_{1, n} \boldsymbol{e}_{r, n}} \\
\underline{\boldsymbol{a}_{2, q} a_{2, n}} \boldsymbol{e}_{r, n}
\end{aligned}
$$

$$
\begin{aligned}
& \hline 3.3 .4 . \widetilde{V}_{1, r} \rightarrow-a_{1, p}\left(-\sum_{j=3}^{n} \sum_{i=1}^{n-1} a_{j, q} a_{j, i} \boldsymbol{e}_{r, i}+\sum_{j=1}^{2} \sum_{i=1}^{n} a_{j, q} \boldsymbol{e}_{j, i} a_{r, i}+\boldsymbol{e}_{r, q}-\sum_{j=1}^{2} a_{j, q} \boldsymbol{f}_{j, r}\right) \\
&+a_{1, p} \delta_{q, n} \boldsymbol{e}_{r, n} \\
&+\left(\sum_{j=2}^{n} a_{j, p} a_{j, q}-\delta_{p, q}\right)\left(-\sum_{i=1}^{n-1} a_{1, i} \boldsymbol{e}_{r, i}-\sum_{i=1}^{n} \boldsymbol{e}_{1, i} a_{r, i}+f_{1, r}\right) \\
&= \sum_{i=1}^{n-1}\left(\sum_{j=3}^{n} a_{1, p} a_{j, q} a_{j, i}-\sum_{j=2}^{n} a_{j, p} a_{j, q} a_{1, i}+\delta_{p, q} a_{1, i}\right) \boldsymbol{e}_{r, i} \\
&-\sum_{i=1}^{n} a_{1, p} a_{2, q} \boldsymbol{e}_{2, i} a_{r, i}-a_{1, p} \boldsymbol{e}_{r, q}+a_{1, p} a_{2, q} \boldsymbol{f}_{2, r}+\delta_{q, n} a_{1, p} \boldsymbol{e}_{r, n} \\
&-\sum_{i, j=1}^{n} \frac{a_{j, p} a_{j, q}}{\boldsymbol{e}_{1, i} a_{r, i}+\sum_{j=1}^{n} a_{j, p} a_{j, q} f_{1, r}+\delta_{p, q} \sum_{i=1}^{n} \boldsymbol{e}_{1, i} a_{r, i}-\delta_{p, q} f_{1, r}} \\
&-\sum_{i=1}^{n} \delta_{p, q} \boldsymbol{e}_{1, i} a_{r, i}+\delta_{p, q} f_{1, r}+\delta_{p, q} \sum_{i=1}^{n} \boldsymbol{e}_{1, i} a_{r, i}-\delta_{p, q} f_{1, r} \\
&-\sum_{i=1}^{n} a_{1, p} a_{2, q} \boldsymbol{e}_{2, i} a_{r, i}-a_{1, p} \boldsymbol{e}_{r, q}+a_{1, p} a_{2, q} \boldsymbol{f}_{2, r}+\delta_{q, n} a_{1, p} \boldsymbol{e}_{r, n} \\
& 3.3 .4 \\
& \sum_{i=1}^{n-1}\left(\sum_{j=3}^{n} a_{1, p} a_{j, q} a_{j, i}-\sum_{j=2}^{n} a_{j, p} a_{j, q} a_{1, i}+\delta_{p, q} a_{1, i}\right)
\end{aligned}
$$


Beginnend mit $\left(a_{1, p} a_{2, q} a_{2, n} \boldsymbol{e}_{r, n}, a_{1, p} a_{2, q} V_{n, q}\right)$ :

$$
\begin{aligned}
& a_{1, p} a_{2, q} a_{2, n} \boldsymbol{e}_{r, n} \\
& \stackrel{\widetilde{V}_{2, q}}{\longrightarrow} a_{1, p} a_{2, q}\left(-\sum_{i=1}^{n-1} a_{2, i} \boldsymbol{e}_{r, i}-\sum_{i=1}^{n} \boldsymbol{e}_{2, i} a_{q, i}+f_{n, q}\right) \\
& =-\sum_{i=1}^{n-1} \underline{a_{1, p} a_{2, q} a_{2, i}} \boldsymbol{e}_{r, i}-\sum_{i=1}^{n} a_{1, p} a_{2, q} \boldsymbol{e}_{2, i} a_{q, i}+a_{1, p} a_{2, q} f_{2, q} \\
& \mid \stackrel{\widetilde{S}_{p, q, i}}{\longrightarrow}-\sum_{i=1}^{n-1}\left(-\sum_{j=3}^{n} a_{1, p} a_{j, q} a_{j, i}+a_{1, p} \delta_{q, i}+\sum_{j=2}^{n} a_{j, p} a_{j, q} a_{1, i}-\delta_{p, q} a_{1, i}\right) \boldsymbol{e}_{r, i} \\
& -\sum_{i=1}^{n} a_{1, p} a_{2, q} \boldsymbol{e}_{2, i} a_{r, i}+a_{1, p} a_{2, q} f_{2, r}
\end{aligned}
$$




\subsubsection{Reduktionswege für $a_{p, 1} a_{n, 1} \boldsymbol{e}_{n, q}$}

Beginnend mit $\left(a_{p, 1} a_{n, 1} \boldsymbol{e}_{n, q}, Z_{p, n} \boldsymbol{e}_{n, q}\right)$ :

$$
\begin{aligned}
\mid \widetilde{\widetilde{Z}_{p, n}} \rightarrow & -\sum_{i=2}^{n} a_{p, i} a_{n, i} \boldsymbol{e}_{n, q}+\delta_{p, n} \boldsymbol{e}_{n, q} \\
\widetilde{\widetilde{W_{i, q}}} & +\sum_{i=2}^{n} \sum_{j=1}^{n-1} a_{p, i} a_{j, i} \boldsymbol{e}_{j, q}-\sum_{j=1}^{n} a_{p, 1} \boldsymbol{e}_{j, 1} a_{j, q}-\boldsymbol{e}_{p, q} \\
& +\sum_{j=1}^{n} \boldsymbol{f}_{p, j} a_{j, q}-\sum_{i=2}^{n} \sum_{k, j=1}^{n} a_{p, i} a_{j, i} \boldsymbol{f}_{j, k} a_{k, q}+\delta_{p, n} \boldsymbol{e}_{n, q}
\end{aligned}
$$

Beginnend mit $\left(a_{p, 1} a_{n, 1} \boldsymbol{e}_{n, q}, a_{p, 1} W_{1, q}\right)$ :

$$
\begin{aligned}
\widetilde{W}_{1, q} & a_{p, 1} \underline{a_{n, 1} \boldsymbol{e}_{n, q}} \\
\hline & -\sum_{j=1}^{n-1} \underline{a_{p, 1} a_{j, 1}} \boldsymbol{e}_{j, q}-\sum_{j=1}^{n} a_{p, 1} \boldsymbol{e}_{j, 1} a_{j, q} \\
& +\sum_{k, j=1}^{n} \underline{a}_{p, 1} a_{j, 1} \boldsymbol{f}_{j, k} a_{k, q} \\
\hline \widetilde{Z}_{p, j}, \widetilde{Z}_{p, j} \longrightarrow & \sum_{j=1}^{n-1} \sum_{i=2}^{n} a_{p, i} a_{j, i} \boldsymbol{e}_{j, q}-\sum_{j=1}^{n-1} \delta_{p, j} \boldsymbol{e}_{j, q}-\sum_{j=1}^{n} a_{p, 1} \boldsymbol{e}_{j, 1} a_{j, q} \\
& -\sum_{k, j=1}^{n} \sum_{i=2}^{n} a_{p, i} a_{j, i} \boldsymbol{f}_{j, k} a_{k, q}+\sum_{k, j=1}^{n} \delta_{p, j} \boldsymbol{f}_{j, k} a_{k, q} \\
= & \sum_{j=1}^{n-1} \sum_{i=2}^{n} a_{p, i} a_{j, i} \boldsymbol{e}_{j, q}-\left(1-\delta_{p, n}\right) \boldsymbol{e}_{p, q}-\sum_{j=1}^{n} a_{p, 1} \boldsymbol{e}_{j, 1} a_{j, q} \\
& -\sum_{k, j=1}^{n} \sum_{i=2}^{n} a_{p, i} a_{j, i} \boldsymbol{f}_{j, k} a_{k, q}+\sum_{k=1}^{n} \boldsymbol{f}_{p, k} a_{k, q}
\end{aligned}
$$


3.3.8 Reduktionswege für $a_{p, 1} a_{q, 2} a_{n, 2} \boldsymbol{e}_{n, r}$

Beginnend mit $\left(a_{n, n} \boldsymbol{e}_{n, n}, Z_{p, q, n} \boldsymbol{e}_{n, r}-Z_{p, q} a_{n, 1} \boldsymbol{e}_{n, r}\right)$ :

$\mid \stackrel{\widetilde{Z}_{p, q, n}}{\longrightarrow}-a_{p, 1} \sum_{i=3}^{n} a_{q, i} a_{n, i} \boldsymbol{e}_{n, r}+a_{p, 1} \delta_{q, n} \boldsymbol{e}_{n, r}+\left(\sum_{i=2}^{n} a_{p, i} a_{q, i}-\delta_{p, q}\right) \underline{a_{n, 1} \boldsymbol{e}_{n, r}}$

3.3.4. $\widetilde{W_{1, r}}$

$$
\left.+\sum_{j=1}^{n} a_{q, 2} \boldsymbol{e}_{j, 2} a_{j, r}+\boldsymbol{e}_{q, r}-\sum_{j=1}^{n} \boldsymbol{f}_{q, j} a_{j, r}+\sum_{i=3}^{n} \sum_{k, j=1}^{n} a_{q, i} a_{j, i} \boldsymbol{f}_{j, k} a_{k, r}\right)
$$

$+a_{p, 1} \delta_{q, n} \boldsymbol{e}_{n, r}+\left(\sum_{i=2}^{n} a_{p, i} a_{q, i}-\delta_{p, q}\right)\left(-\sum_{j=1}^{n-1} a_{j, 1} \boldsymbol{e}_{j, r}\right.$

$\left.-\sum_{j=1}^{n} \boldsymbol{e}_{j, 1} a_{j, r}+\sum_{k, j=1}^{n} a_{j, 1} \boldsymbol{f}_{j, k} a_{k, r}\right)$

$=\sum_{i=3}^{n} \sum_{j=1}^{n-1} a_{p, 1} a_{q, i} a_{j, i} \boldsymbol{e}_{j, r}-\sum_{j=1}^{n} \underline{a_{p, 1} a_{q, 1}} \boldsymbol{e}_{j, 1} a_{j, r}$

$-\sum_{j=1}^{n} a_{p, 1} a_{q, 2} \boldsymbol{e}_{j, 2} a_{j, r}-a_{p, 1} \boldsymbol{e}_{q, r}+\sum_{j=1}^{n} a_{p, 1} \boldsymbol{f}_{q, j} a_{j, r}$

$-\sum_{i=3}^{n} \sum_{k, j=1}^{n} a_{p, 1} a_{q, i} a_{j, i} \boldsymbol{f}_{j, k} a_{k, r}+a_{p, 1} \delta_{q, n} \boldsymbol{e}_{n, r}$

$-\sum_{i=2}^{n} \sum_{j=1}^{n-1} a_{p, i} a_{q, i} a_{j, 1} \boldsymbol{e}_{j, r}-\sum_{i=2}^{n} \sum_{j=1}^{n} a_{p, i} a_{q, i} \boldsymbol{e}_{j, 1} a_{j, r}$

$+\sum_{i=2}^{n} \sum_{k, j=1}^{n} a_{p, i} a_{q, i} a_{j, 1} \boldsymbol{f}_{j, k} a_{k, r}$

$+\delta_{p, q}\left(\sum_{j=1}^{n-1} a_{j, 1} \boldsymbol{e}_{j, r}+\sum_{j=1}^{n} \boldsymbol{e}_{j, 1} a_{j, r}-\sum_{k, j=1}^{n} a_{j, 1} \boldsymbol{f}_{j, k} a_{k, r}\right)$ 


$$
\begin{aligned}
\widetilde{Z}_{p, q} & \sum_{i=3}^{n} \sum_{j=1}^{n-1} a_{p, 1} a_{q, i} a_{j, i} \boldsymbol{e}_{j, r} \\
& +\sum_{j=1}^{n} \sum_{i=2}^{n} a_{p, i} a_{q, i} \boldsymbol{e}_{j, 1} a_{j, r}-\sum_{j=1}^{n} \delta_{p, q} \boldsymbol{e}_{j, 1} a_{j, r} \\
& -\sum_{j=1}^{n} a_{p, 1} a_{q, 2} \boldsymbol{e}_{j, 2} a_{j, r}-a_{p, 1} \boldsymbol{e}_{q, r}+\sum_{j=1}^{n} a_{p, 1} \boldsymbol{f}_{q, j} a_{j, r} \\
& -\sum_{i=3}^{n} \sum_{k, j=1}^{n} a_{p, 1} a_{q, i} a_{j, i} \boldsymbol{f}_{j, k} a_{k, r}+a_{p, 1} \delta_{q, n} \boldsymbol{e}_{n, r} \\
& -\sum_{i=2}^{n} \sum_{j=1}^{n-1} a_{p, i} a_{q, i} a_{j, 1} \boldsymbol{e}_{j, r}-\sum_{i=2}^{n} \sum_{j=1}^{n} a_{p, i} a_{q, i} \boldsymbol{e}_{j, 1} a_{j, r} \\
& +\sum_{i=2}^{n} \sum_{k, j=1}^{n} a_{p, i} a_{q, i} a_{j, 1} \boldsymbol{f}_{j, k} a_{k, r} \\
& +\sum_{j=1}^{n} a_{p, q, j} \boldsymbol{f}_{j, k} a_{k, r}-\sum_{k, j=1}^{n} a_{q, 2} \boldsymbol{e}_{j, 2} a_{j, r} \\
& +\sum_{p, q}\left(\sum_{j=1}^{n-1} a_{j, 1} \boldsymbol{e}_{j, r}+\sum_{j=1}^{n} \boldsymbol{e}_{j, 1} a_{j, r} a_{k, r}-\sum_{k, j=1}^{n} a_{j, 1} \boldsymbol{f}_{j, k} a_{k, r}\right. \\
& +\sum_{j=1}^{n} a_{p, 1} \boldsymbol{f}_{q, j} a_{j, r} \boldsymbol{e}_{j, r}+\sum_{k, j=1}^{n} Z_{p, q} a_{j, 1} \boldsymbol{e}_{j, r} \\
& -\sum_{i=3}^{n} \sum_{k, j=1}^{n} a_{p, 1} a_{q, i} a_{j, i} \boldsymbol{f}_{j, k} a_{k, r} \\
& +\sum_{i=}^{n} \sum_{j=1}^{n} a_{p, 1} a_{q, i} a_{j, i} \boldsymbol{e}_{j, r}-a_{p, 1}\left(1-\delta_{q, n}\right) \boldsymbol{e}_{q, r} \\
& \left.+a_{j=1} a_{p, i} a_{q, i}-\delta_{p, q}\right) a_{j, 1} \boldsymbol{e}_{j, r}-\sum_{j=1}^{n} a_{p, 1} a_{q, 2} \boldsymbol{e}_{j, 2} a_{j, r} a_{k, r}
\end{aligned}
$$


Beginnend mit $\left(a_{p, 1} a_{q, 2} a_{n, 2} \boldsymbol{e}_{n, r}, a_{p, 1} a_{q, 2} W_{2, r}\right)$ :

$$
\begin{aligned}
\widetilde{W}_{2, r} & a_{p, 1} a_{q, 2} \\
\longrightarrow & -\sum_{n, 2}^{n-1} \boldsymbol{e}_{n, r} \\
a_{p, 1} a_{q, 2} a_{j, 2} & \boldsymbol{e}_{j, r}-\sum_{j=1}^{n} a_{p, 1} a_{q, 2} \boldsymbol{e}_{j, 2} a_{j, r}+\sum_{k, j=1}^{n} \frac{a_{p, 1} a_{q, 2} a_{j, 2}}{\boldsymbol{f}_{j, k}} a_{k, r} \\
= & -\sum_{j=1}^{n-1} Z_{p, q, j} \boldsymbol{e}_{j, r}+\sum_{j=1}^{n-1} Z_{p, q} a_{j, 1} \boldsymbol{e}_{j, r} \\
& -\sum_{j=1}^{n} a_{p, 1} a_{q, 2} \boldsymbol{e}_{j, 2} a_{j, r} \\
& +\sum_{k, j=1}^{n} Z_{p, q, j} \boldsymbol{f}_{j, k} a_{k, r}-\sum_{k, j=1}^{n} Z_{p, q} a_{j, 1} \boldsymbol{f}_{j, k} a_{k, r}
\end{aligned}
$$




\subsubsection{Reduktionswege für $a_{n, n} \boldsymbol{e}_{n, n}$}

Beginnend mit $\left(a_{n, n} \boldsymbol{e}_{n, n}, W_{n, n}\right)$ :

$$
\begin{aligned}
& \underline{a_{n, n} \boldsymbol{e}_{n, n}} \\
& \mid \stackrel{\widetilde{W}_{n, n}}{\longrightarrow}-\sum_{j=1}^{n-1} \underline{a_{j, n} \boldsymbol{e}_{j, n}}-\sum_{j=1}^{n} \boldsymbol{e}_{j, n} a_{j, n}+\sum_{k, j=1}^{n} a_{j, n} \boldsymbol{f}_{j, k} a_{k, n} \\
& \stackrel{\tilde{V}_{j, j}}{\longrightarrow}-\sum_{j=1}^{n-1}\left(-\sum_{i=1}^{n-1} a_{j, i} \boldsymbol{e}_{j, i}-\sum_{i=1}^{n} \boldsymbol{e}_{j, i} a_{j, i}+\boldsymbol{f}_{j, j}\right) \\
& -\sum_{j=1}^{n} \boldsymbol{e}_{j, n} a_{j, n}+\sum_{k, j=1}^{n} a_{j, n} \boldsymbol{f}_{j, k} a_{k, n} \\
& =\sum_{i, j=1}^{n-1} a_{j, i} \boldsymbol{e}_{j, i}+\sum_{i, j=1}^{n-1} \boldsymbol{e}_{j, i} a_{j, i}-\boldsymbol{e}_{n, n} a_{n, n}-\sum_{j=1}^{n-1} \boldsymbol{f}_{j, j} \\
& +\sum_{k, j=1}^{n} a_{j, n} \boldsymbol{f}_{j, k} a_{k, n}
\end{aligned}
$$


Beginnend mit $\left(a_{n, n} \boldsymbol{e}_{n, n}, V_{n, n}\right)$ :

$$
\begin{aligned}
& \underline{a_{n, n} \boldsymbol{e}_{n, n}} \\
& \mid \stackrel{\widetilde{V}_{n, n}}{\longrightarrow}-\sum_{i=1}^{n-1} \frac{a_{n, i} \boldsymbol{e}_{n, i}}{\longrightarrow}-\sum_{i=1}^{n} \boldsymbol{e}_{n, i} a_{n, i}+\boldsymbol{f}_{n, n} \\
& \mid \stackrel{\widetilde{W}_{i, i}}{\longrightarrow}-\sum_{i=1}^{n-1}\left(-\sum_{j=1}^{n-1} a_{j, i} \boldsymbol{e}_{j, i}-\sum_{j=1}^{n} \boldsymbol{e}_{j, i} a_{j, i}+\sum_{k, j=1}^{n} a_{j, i} \boldsymbol{f}_{j, k} a_{k, i}\right) \\
& -\sum_{i=1}^{n} \boldsymbol{e}_{n, i} a_{n, i}+\boldsymbol{f}_{n, n} \\
& =\sum_{i, j=1}^{n-1} a_{j, i} \boldsymbol{e}_{j, i}+\sum_{i, j=1}^{n-1} \boldsymbol{e}_{j, i} a_{j, i}-\boldsymbol{e}_{n, n} a_{n, n}+\boldsymbol{f}_{n, n} \\
& -\sum_{i=2}^{n-1} \sum_{k, j=1}^{n} a_{j, i} \boldsymbol{f}_{j, k} a_{k, i}-\sum_{\substack{k, j=1 \\
(k, j) \neq(n, n)}}^{n} a_{j, 1} \boldsymbol{f}_{j, k} a_{k, 1} \\
& -\underline{a_{n, 1} \boldsymbol{f}_{n, n} a_{n, 1}} \\
& \stackrel{\widetilde{K}}{\longrightarrow} \sum_{i, j=1}^{n-1} a_{j, i} \boldsymbol{e}_{j, i}+\sum_{i, j=1}^{n-1} \boldsymbol{e}_{j, i} a_{j, i}-\boldsymbol{e}_{n, n} a_{n, n}+\boldsymbol{f}_{n, n} \\
& -\sum_{i=2}^{n-1} \sum_{k, j=1}^{n} a_{j, i} \boldsymbol{f}_{j, k} a_{k, i}-\sum_{\substack{k, j=1 \\
k, j) \neq(n, n)}}^{n} a_{j, 1} \boldsymbol{f}_{j, k} a_{k, 1} \\
& +\sum_{\substack{i, j, k=1 \\
(i, j, k) \neq(1, n, n)}}^{n} a_{j, i} \boldsymbol{f}_{j, k} a_{k, i}-\sum_{i=1}^{n} \boldsymbol{f}_{i, i} \\
& =\sum_{i, j=1}^{n-1} a_{j, i} \boldsymbol{e}_{j, i}+\sum_{i, j=1}^{n-1} \boldsymbol{e}_{j, i} a_{j, i}-\boldsymbol{e}_{n, n} a_{n, n}-\sum_{i=1}^{n-1} \boldsymbol{f}_{i, i} \\
& +\sum_{k, j=1}^{n} a_{j, n} \boldsymbol{f}_{j, k} a_{k, n}
\end{aligned}
$$




\section{$3.4 \Phi_{3}$}

\subsection{1 Übersichtstabelle für $\Phi_{3}$}

Um die Lesbarkeit zu erhöhen setzen wir:

$$
Q:=\sum_{i=3}^{n} \sum_{j, k=1}^{n} a_{j, i} \boldsymbol{f}_{j, k} a_{k, i}-\sum_{i=1}^{n} \boldsymbol{f}_{i, i}-\boldsymbol{f} .
$$

\begin{tabular}{|c|c|}
\hline & $\Phi_{3}: \mathcal{A}^{e}(n) \rightarrow \mathcal{A}^{e}(n)^{n^{2}}$ \\
\hline Matrix & $\boldsymbol{f} \mapsto-\operatorname{tr}\left(A^{t} \boldsymbol{F} A\right)+\operatorname{tr}(\boldsymbol{F})$ \\
\hline $\begin{array}{l}\text { Kompo- } \\
\text { nenten }\end{array}$ & $\boldsymbol{f} \mapsto-\sum_{i, j, k=1}^{n} a_{j, i} \boldsymbol{f}_{j, k} a_{k, i}+\sum_{i=1}^{n} \boldsymbol{f}_{i, i}$ \\
\hline$r_{\Phi_{3}}$ & $\widetilde{V}=\left(a_{n, 1} \boldsymbol{f}_{n, n} a_{n, 1},-Q-\sum_{j, k=1}^{n} a_{j, 2} \boldsymbol{f}_{j, k} a_{k, 2}-\sum_{\substack{j, k=1 \\
(j, k) \neq(n, n)}}^{n} a_{j, 1} \boldsymbol{f}_{j, k} a_{k, 1}\right.$ \\
\hline$r_{e s}$ & 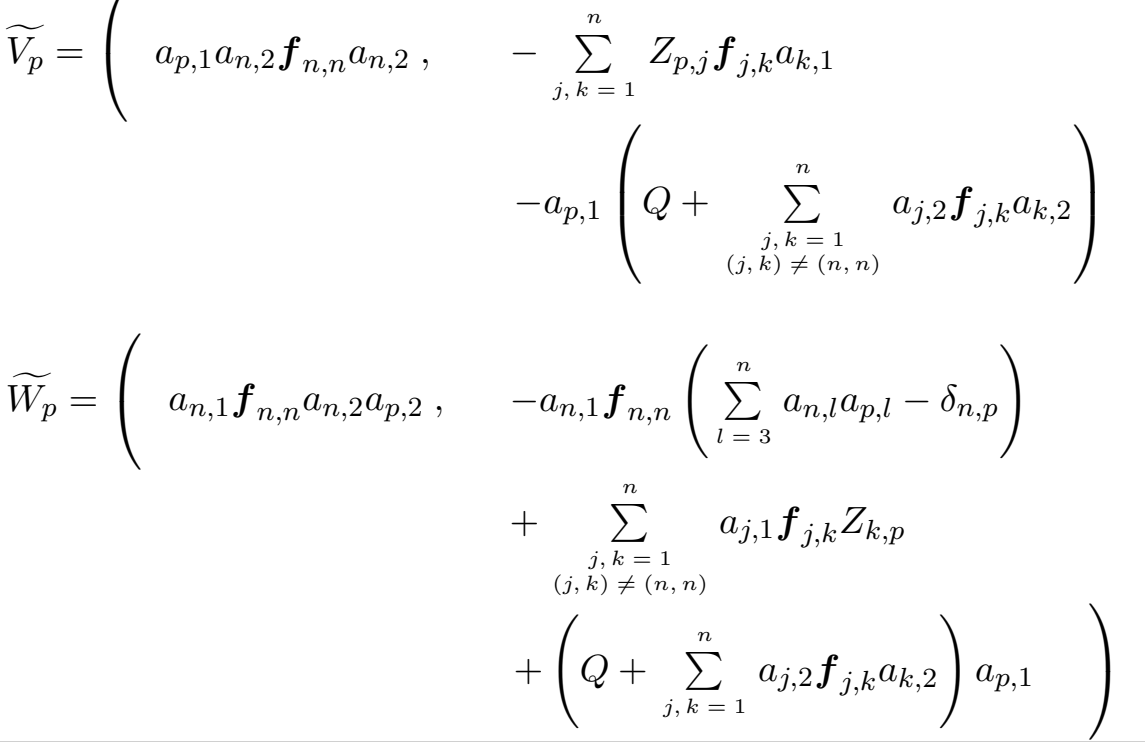 \\
\hline \multicolumn{2}{|l|}{$r_{\text {kern }}$} \\
\hline Konflikte & siehe Tabelle 3.4 .3 \\
\hline
\end{tabular}




\subsection{2 $r_{e s}$}

Wir werden zeigen, dass $S_{e s} \subset\left\langle S_{\Phi_{3}}\right\rangle$. Dazu betrachten wir $S_{\Phi_{3}}$ :

$$
a_{n, 1} \boldsymbol{f}_{n, n} a_{n, 1}-\left(-Q-\sum_{j, k=1}^{n} a_{j, 2} \boldsymbol{f}_{j, k} a_{k, 2}-\sum_{\substack{j, k=1 \\ j, k) \neq(n, n)}}^{n} a_{j, 1} \boldsymbol{f}_{j, k} a_{k, 1}\right) .
$$

Wenn wir von links mit $a_{p, 1}$ multiplizieren, erhalten wir:

$$
\begin{gathered}
\underline{a_{p, 1} a_{n, 1} \boldsymbol{f}_{n, n} a_{n, 1}+a_{p, 1} Q+a_{p, 1}} \sum_{j, k=1}^{n} a_{j, 2} \boldsymbol{f}_{j, k} a_{k, 2}+\sum_{\substack{j, k=1 \\
(j, k) \neq(n, n)}}^{n} a_{p, 1} a_{j, 1} \boldsymbol{f}_{j, k} a_{k, 1} \\
=a_{p, 1} a_{n, 2} \boldsymbol{f}_{n, n} a_{n, 2}+a_{p, 1} Q+a_{p, 1} \sum_{\substack{j, k=1 \\
(j, k) \neq(n, n)}}^{n} a_{j, 2} \boldsymbol{f}_{j, k} a_{k, 2}+\sum_{j, k=1}^{n} Z_{p, j} \boldsymbol{f}_{j, k} a_{k, 1} .
\end{gathered}
$$

Das entspricht genau dem Teil von $S_{e s}$, der von $\widetilde{V}_{p}$ induziert ist.

Wenn wir von rechts mit $a_{p, 1}$ multiplizieren, erhalten wir:

$$
\begin{aligned}
& a_{n, 1} \boldsymbol{f}_{n, n} \underline{a_{n, 1} a_{p, 1}}+Q a_{p, 1}+\sum_{j, k=1}^{n} a_{j, 2} \boldsymbol{f}_{j, k} a_{k, 2} a_{p, 1}+\sum_{\substack{j, k=1 \\
(j, k) \neq(n, n)}}^{n} a_{j, 1} \boldsymbol{f}_{j, k} \underline{a_{k, 1} a_{p, 1}} \\
= & a_{n, 1} \boldsymbol{f}_{n, n} a_{n, 2} a_{p, 2}+a_{n, 1} \boldsymbol{f}_{n, n}\left(\sum_{l=3}^{n} a_{n, l} a_{p, l}+\delta_{n, p}\right) \\
& -\left(Q+\sum_{j, k=1}^{n} a_{j, 2} \boldsymbol{f}_{j, k} a_{k, 2}\right) a_{p, 1}-\sum_{\substack{j, k=1 \\
(j, k) \neq(n, n)}}^{n} a_{j, 1} \boldsymbol{f}_{j, k} Z_{k, p} .
\end{aligned}
$$

Das entspricht genau dem Teil von $S_{e s}$, der von $\widetilde{W}_{p}$ induziert ist.

Zusammen folgt: $S_{e s} \subset\left\langle S_{\Phi_{3}}\right\rangle$.

\subsubsection{Konflikte:}

Wir wollen nun eine Tabelle aller möglichen Konflikte betrachten. Dazu überlegen wir uns zunächst, dass es keine Konflikte innerhalb von $r_{e}$ gibt. Dazu müsste es eine Überschneidung geben, so dass der Erzeuger des Moduls $\boldsymbol{f}_{n, n}$ in dem Wort nur einmal vorkommt. Dies ist aber durch die festgesetzten Indizes nicht möglich.

Zwischen den Regeln aus $r_{\boldsymbol{A}}$ und denen aus $r_{e}$ kann es Überschneidungen geben. Anders als in den vorhergehenden Fällen können die Regeln aus $r_{\boldsymbol{A}}$ sogar rechts von denen aus $r_{e}$ vorkommen. Es kommen Überschneidungen mit einem oder mit zwei Buchstaben vor, in den meisten Fällen ist jedoch durch die festgesetzten Indizes keine Überschneidung möglich. In der folgenden Tabelle stehen alle minimalen Überschneidungen, wobei oberhalb des Striches die Regeln aus $r_{\boldsymbol{A}}$ von links und unterhalb von rechts angewendet werden. Wenn es Überschneidungen mit mehr als einem Buchstaben gibt, stehen beide Fälle übereinander. 


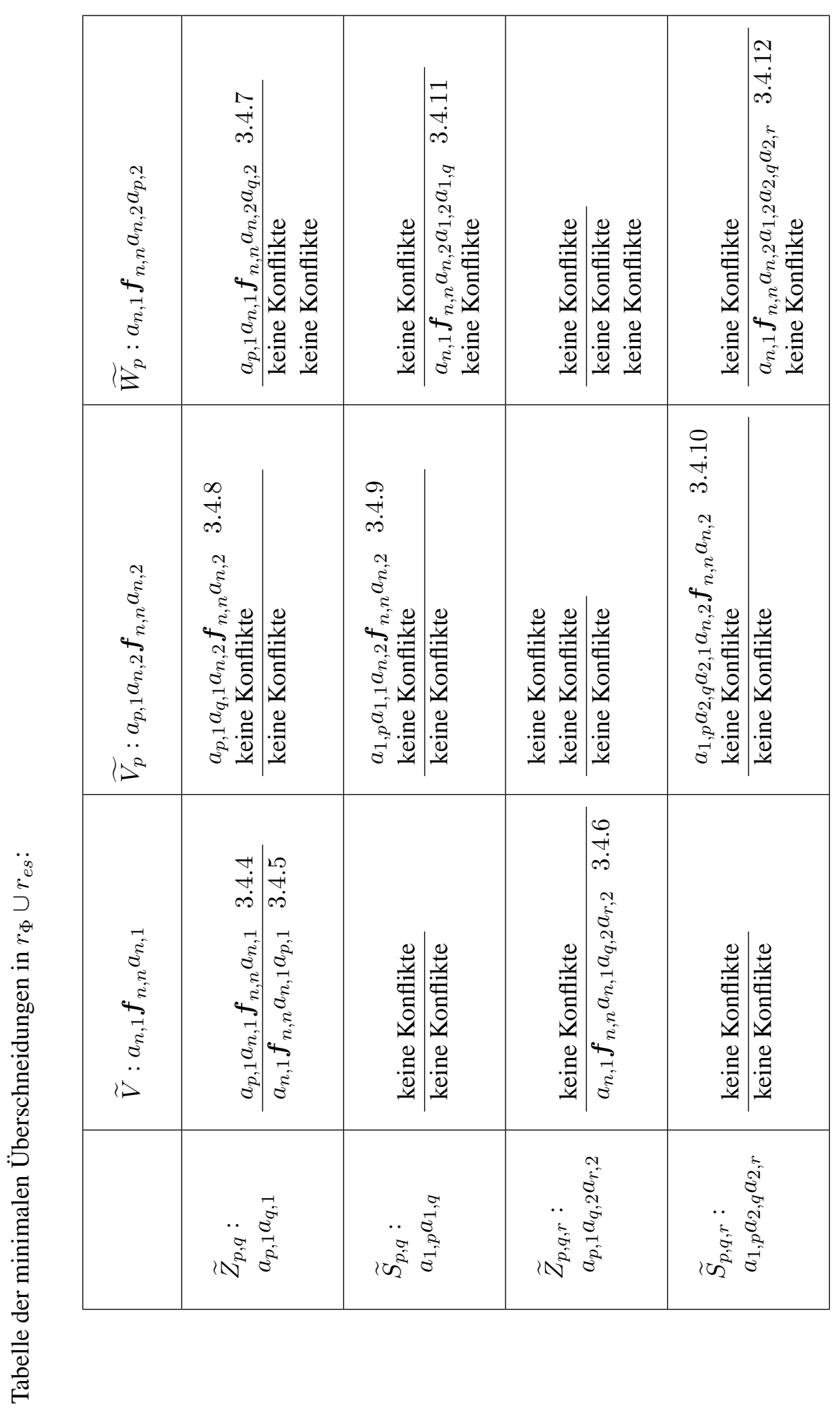


3.4.4 Reduktionswege für $a_{p, 1} a_{n, 1} \boldsymbol{f}_{n, n} a_{n, 1}$

Beginnend mit $\left(a_{p, 1} a_{n, 1} \boldsymbol{f}_{n, n} a_{n, 1}, a_{p, 1} V\right)$ :

$$
\begin{aligned}
& a_{p, 1} \underline{a_{n, 1} \boldsymbol{f}_{n, n} a_{n, 1}} \\
& \mid \stackrel{\widetilde{V}}{\longrightarrow}-a_{p, 1} Q-\sum_{j, k=1}^{n} a_{p, 1} a_{j, 2} \boldsymbol{f}_{j, k} a_{k, 2}-\sum_{\substack{j, k=1 \\
j, k \neq \neq(n, n)}}^{n} \underline{a_{p, 1} a_{j, 1}} \boldsymbol{f}_{j, k} a_{k, 1} \\
& \mid \stackrel{\widetilde{Z}_{p, j}}{\longrightarrow}-a_{p, 1} Q-\sum_{j, k=1}^{n} a_{p, 1} a_{j, 2} \boldsymbol{f}_{j, k} a_{k, 2}-\sum_{\substack{j, k=1 \\
j, k) \neq(n, n)}}^{n} Z_{p, j} \boldsymbol{f}_{j, k} a_{k, 1} \\
& =-a_{p, 1} Q-\sum_{\substack{j, k=1 \\
(j, k) \neq(n, n)}}^{n} a_{p, 1} a_{j, 2} \boldsymbol{f}_{j, k} a_{k, 2}-\underline{a_{p, 1} a_{n, 2} \boldsymbol{f}_{n, n} a_{n, 2}} \\
& -\sum_{\substack{j, k=1 \\
(j, k) \neq(n, n)}}^{n} Z_{p, j} \boldsymbol{f}_{j, k} a_{k, 1} \\
& \mid \stackrel{\widetilde{V}_{p}}{\longrightarrow}-a_{p, 1} Q-\sum_{\substack{j, k=1 \\
(j, k) \neq(n, n)}}^{n} a_{p, 1} a_{j, 2} \boldsymbol{f}_{j, k} a_{k, 2} \\
& -\left(-\sum_{j, k=1}^{n} Z_{p, j} \boldsymbol{f}_{j, k} a_{k, 1}-a_{p, 1}\left(Q+\sum_{\substack{j, k=1 \\
(j, k) \neq(n, n)}}^{n} a_{j, 2} \boldsymbol{f}_{j, k} a_{k, 2}\right)\right) \\
& -\sum_{\substack{j, k=1 \\
(j, k) \neq(n, n)}}^{n} Z_{p, j} \boldsymbol{f}_{j, k} a_{k, 1} \\
& =Z_{p, n} \boldsymbol{f}_{n, n} a_{n, 1}
\end{aligned}
$$

Beginnend mit $\left(a_{p, 1} a_{n, 1} \boldsymbol{f}_{n, n} a_{n, 1}, Z_{p, n} \boldsymbol{f}_{n, n} a_{n, 1}\right)$ :

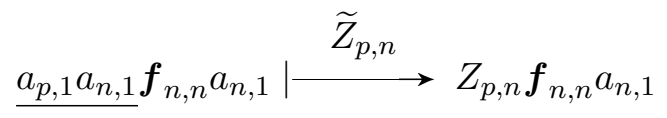




\subsubsection{Reduktionswege für $a_{n, 1} \boldsymbol{f}_{n, n} a_{n, 1} a_{q, 1}$}

Beginnend mit $\left(a_{n, 1} \boldsymbol{f}_{n, n} a_{n, 1} a_{q, 1}, V a_{q, 1}\right)$ :

$$
\begin{gathered}
\stackrel{\widetilde{V}}{\longrightarrow}-Q a_{q, 1}-\sum_{j, k=1}^{n} a_{j, 2} \boldsymbol{f}_{j, k} a_{k, 2} a_{q, 1}-\sum_{\substack{j, k=1 \\
(j, k) \neq(n, n)}}^{n} a_{j, 1} \boldsymbol{f}_{j, k} a_{k, 1} a_{q, 1} \\
\stackrel{\boldsymbol{f}_{n, n} a_{n, 1} a_{q, 1}}{\longrightarrow}-Q a_{q, 1}-\sum_{j, k=1}^{n} a_{j, 2} \boldsymbol{f}_{j, k} a_{k, 2} a_{q, 1}-\sum_{\substack{j, k=1 \\
j, k \neq \neq(n, n)}}^{n} a_{j, 1} \boldsymbol{f}_{j, k} Z_{k, q}
\end{gathered}
$$

Beginnend mit $\left(a_{n, 1} \boldsymbol{f}_{n, n} a_{n, 1} a_{q, 1}, a_{n, 1} \boldsymbol{f}_{n, n}\left(Z_{n, q}\right)\right)$ :

$$
\begin{aligned}
& a_{n, 1} \boldsymbol{f}_{n, n} \underline{a_{n, 1} a_{q, 1}} \\
\hline \widetilde{Z}_{n, q} \longrightarrow & a_{n, 1} \boldsymbol{f}_{n, n} Z_{n, q} \\
= & -\frac{a_{n, 1} \boldsymbol{f}_{n, n} a_{n, 2} a_{q, 2}}{\longrightarrow} \sum_{i=3}^{n} a_{n, 1} \boldsymbol{f}_{n, n} a_{n, i} a_{q, i}+\delta_{n, q} a_{n, 1} \boldsymbol{f}_{n, n} \\
\widetilde{W}_{q} \longrightarrow & a_{n, 1} \boldsymbol{f}_{n, n}\left(\sum_{l=3}^{n} a_{n, l} a_{q, l}-\delta_{n, q}\right)-\sum_{\substack{j, k=1 \\
(j, k) \neq(n, n)}}^{n} a_{j, 1} \boldsymbol{f}_{j, k} Z_{k, q} \\
& -\left(\sum_{j, k=1}+\sum_{j, 2}^{n} \boldsymbol{f}_{j, k} a_{k, 2}\right) a_{q, 1} \sum_{\substack{j=3 \\
n}}^{n} a_{n, 1} \boldsymbol{f}_{n, n} a_{n, i} a_{q, i}+\delta_{n, q} a_{n, 1} \boldsymbol{f}_{n, n} \\
= & -\sum_{\substack{j, k=1 \\
(j, k) \neq(n, n)}}^{n} a_{j, 1} \boldsymbol{f}_{j, k} Z_{k, q}-Q a_{q, 1}-\sum_{j, k=1}^{n} a_{j, 2} \boldsymbol{f}_{j, k} a_{k, 2} a_{q, 1}
\end{aligned}
$$


3.4.6 Reduktionswege für $a_{n, 1} \boldsymbol{f}_{n, n} a_{n, 1} a_{q, 2} a_{r, 2}$

Beginnend mit $\left(a_{n, 1} \boldsymbol{f}_{n, n} a_{n, 1} a_{q, 2} a_{r, 2}, V a_{q, 2} a_{r, 2}\right)$ :

$$
\begin{aligned}
& \mid \stackrel{\widetilde{V}}{\longrightarrow}-Q a_{q, 2} a_{r, 2}-\sum_{j, k=1}^{n} a_{j, 2} \boldsymbol{f}_{j, k} a_{k, 2} a_{q, 2} a_{r, 2} \\
&-\sum_{\substack{j, k=1 \\
j, k) \neq(n, n)}}^{n} a_{j, 1} \boldsymbol{f}_{j, k} \underline{a_{k, 1} a_{q, 2} a_{r, 2}} \\
& \hline \widetilde{Z}_{k, q, r} a_{n, 1} a_{q, 2} a_{r, 2} \\
& \longrightarrow-Q a_{q, 2} a_{r, 2}-\sum_{j, k=1}^{n} a_{j, 2} \boldsymbol{f}_{j, k} a_{k, 2} a_{q, 2} a_{r, 2} \\
&-\sum_{\substack{j, k=1 \\
(j, k) \neq(n, n)}}^{n} a_{j, 1} \boldsymbol{f}_{j, k}\left(Z_{k, q, r}-Z_{k, q} a_{r, 1}\right)
\end{aligned}
$$

Beginnend mit $\left(a_{n, 1} \boldsymbol{f}_{n, n} a_{n, 1} a_{q, 2} a_{r, 2}, a_{n, 1} \boldsymbol{f}_{n, n}\left(Z_{n, q, r}-Z_{n, q} a_{r, 1}\right)\right)$ :

$$
\begin{aligned}
& a_{n, 1} \boldsymbol{f}_{n, n} \underline{a_{n, 1} a_{q, 2} a_{r, 2}} \\
& \mid \stackrel{\widetilde{Z}_{n, q, r}}{\longrightarrow}-\underline{a_{n, 1} \boldsymbol{f}_{n, n} a_{n, 1}}\left(\sum_{l=3}^{n} a_{q, i} a_{r, i}-\delta_{q, r}\right) \\
& +a_{n, 1} \boldsymbol{f}_{n, n}\left(\sum_{i=2}^{n} a_{n, i} a_{q, i}-\delta_{n, q}\right) a_{r, 1} \\
& \stackrel{\widetilde{V}}{\longrightarrow}\left(Q+\sum_{j, k=1}^{n} a_{j, 2} \boldsymbol{f}_{j, k} a_{k, 2}\right. \\
& \left.+\sum_{\substack{j, k=1 \\
(j, k) \neq(n, n)}}^{n} a_{j, 1} \boldsymbol{f}_{j, k} a_{k, 1}\right)\left(\sum_{l=3}^{n} a_{q, l} a_{r, l}-\delta_{q, r}\right) \\
& +\underline{a_{n, 1} \boldsymbol{f}_{n, n} a_{n, 2} a_{q, 2}} a_{r, 1}+a_{n, 1} \boldsymbol{f}_{n, n}\left(\sum_{i=3}^{n} a_{n, i} a_{q, i}-\delta_{n, q}\right) a_{r, 1}
\end{aligned}
$$




$$
\begin{aligned}
& \stackrel{\widetilde{W}_{q}}{\longrightarrow}\left(Q+\sum_{j, k=1}^{n} a_{j, 2} \boldsymbol{f}_{j, k} a_{k, 2}\right. \\
& \left.+\sum_{\substack{j, k=1 \\
(j, k) \neq(n, n)}}^{n} a_{j, 1} \boldsymbol{f}_{j, k} a_{k, 1}\right)\left(\sum_{l=3}^{n} a_{q, l} a_{r, l}-\delta_{q, r}\right) \\
& -a_{n, 1} \boldsymbol{f}_{n, n}\left(\sum_{l=3}^{n} a_{n, l} a_{q, l}-\delta_{n, q}\right) a_{r, 1} \\
& +\sum_{\substack{j, k=1 \\
(j, k) \neq(n, n)}}^{n} a_{j, 1} \boldsymbol{f}_{j, k} Z_{k, q} a_{r, 1} \\
& +\left(Q+\sum_{j, k=1}^{n} a_{j, 2} \boldsymbol{f}_{j, k} a_{k, 2}\right) \underline{a_{q, 1} a_{r, 1}} \\
& +a_{n, 1} \boldsymbol{f}_{n, n}\left(\sum_{i=3}^{n} a_{n, i} a_{q, i}-\delta_{n, q}\right) a_{r, 1} \\
& \stackrel{\widetilde{Z}_{q, r}}{\longrightarrow}\left(Q+\sum_{j, k=1}^{n} a_{j, 2} \boldsymbol{f}_{j, k} a_{k, 2}\right)\left(\sum_{l=3}^{n} a_{q, l} a_{r, l}-\delta_{q, r}\right) \\
& +\sum_{\substack{j, k=1 \\
(j, k) \neq(n, n)}}^{n} a_{j, 1} \boldsymbol{f}_{j, k} a_{k, 1}\left(\sum_{l=3}^{n} a_{q, l} a_{r, l}-\delta_{q, r}\right) \\
& +\sum_{\substack{j, k=1 \\
(j, k) \neq(n, n)}}^{n} a_{j, 1} \boldsymbol{f}_{j, k} Z_{k, q} a_{r, 1} \\
& +\left(Q+\sum_{j, k=1}^{n} a_{j, 2} \boldsymbol{f}_{j, k} a_{k, 2}\right) Z_{q, r} \\
& =-\left(Q+\sum_{j, k=1}^{n} a_{j, 2} \boldsymbol{f}_{j, k} a_{k, 2}\right) a_{q, 2} a_{r, 2} \\
& \text { - } \sum_{\substack{j, k=1 \\
(j, k) \neq(n, n)}}^{n} a_{j, 1} \boldsymbol{f}_{j, k}\left(Z_{k, q, r}-Z_{k, q} a_{r, 1}\right)
\end{aligned}
$$


3.4.7 Reduktionswege für $a_{p, 1} a_{n, 1} \boldsymbol{f}_{n, n} a_{n, 2} a_{q, 2}$

Beginnend mit $\left(a_{p, 1} a_{n, 1} \boldsymbol{f}_{n, n} a_{n, 2} a_{q, 2}, a_{p, 1} W_{q}\right)$ :

$$
\begin{aligned}
& a_{p, 1} \underline{a_{n, 1} \boldsymbol{f}_{n, n} a_{n, 2} a_{q, 2}} \\
& \mid \stackrel{\widetilde{W}_{q}}{\longrightarrow}-\underline{a_{p, 1} a_{n, 1}} \boldsymbol{f}_{n, n}\left(\sum_{l=3}^{n} a_{n, l} a_{q, l}-\delta_{n, q}\right) \\
& +\sum_{\substack{j, k=1 \\
(j, k) \neq(n, n)}}^{n} \underline{a_{p, 1} a_{j, 1}} \boldsymbol{f}_{j, k} Z_{k, q} \\
& +a_{p, 1}\left(Q+\sum_{j, k=1}^{n} a_{j, 2} \boldsymbol{f}_{j, k} a_{k, 2}\right) a_{q, 1} \\
& \mid \stackrel{\widetilde{Z}_{p, n}, \widetilde{Z}_{p, j}}{\longrightarrow}-Z_{p, n} \boldsymbol{f}_{n, n}\left(\sum_{l=3}^{n} a_{n, l} a_{q, l}-\delta_{n, q}\right) \\
& +\sum_{\substack{j, k=1 \\
(j, k) \neq(n, n)}}^{n} Z_{p, j} \boldsymbol{f}_{j, k} Z_{k, q} \\
& +a_{p, 1}\left(Q+\sum_{\substack{j, k=1 \\
(j, k) \neq(n, n)}}^{n} a_{j, 2} \boldsymbol{f}_{j, k} a_{k, 2}\right) a_{q, 1} \\
& +\underline{a_{p, 1} a_{n, 2} \boldsymbol{f}_{n, n} a_{n, 2}} a_{q, 1} \\
& \mid \stackrel{\widetilde{V}_{p}}{\longrightarrow}+\sum_{j, k=1}^{n} Z_{p, j} \boldsymbol{f}_{j, k} Z_{k, q}+Z_{p, n} \boldsymbol{f}_{n, n} a_{n, 2} a_{q, 2} \\
& +a_{p, 1}\left(Q+\sum_{\substack{j, k=1 \\
(j, k) \neq(n, n)}}^{n} a_{j, 2} \boldsymbol{f}_{j, k} a_{k, 2}\right) a_{q, 1} \\
& -\sum_{j, k=1}^{n} Z_{p, j} \boldsymbol{f}_{j, k} \underline{a_{k, 1} a_{q, 1}} \\
& -a_{p, 1}\left(Q+\sum_{\substack{j, k=1 \\
(j, k) \neq(n, n)}}^{n} a_{j, 2} \boldsymbol{f}_{j, k} a_{k, 2}\right) a_{q, 1}
\end{aligned}
$$




$$
\begin{aligned}
\mid \stackrel{\widetilde{Z}_{k, q}}{\longrightarrow} & +\sum_{j, k=1}^{n} Z_{p, j} \boldsymbol{f}_{j, k} Z_{k, q}+Z_{p, n} \boldsymbol{f}_{n, n} a_{n, 2} a_{q, 2} \\
& -\sum_{j, k=1}^{n} Z_{p, j} \boldsymbol{f}_{j, k} Z_{k, q} \\
= & Z_{p, n} \boldsymbol{f}_{n, n} a_{n, 2} a_{q, 2}
\end{aligned}
$$

Beginnend mit $\left(a_{p, 1} a_{n, 1} \boldsymbol{f}_{n, n} a_{n, 2} a_{q, 2}, Z_{p, n} \boldsymbol{f}_{n, n} a_{n, 2} a_{q, 2}\right)$ :

$$
\underline{a_{p, 1} a_{n, 1}} \boldsymbol{f}_{n, n} a_{n, 2} a_{q, 2} \mid \stackrel{\widetilde{Z}_{p, n}}{\longrightarrow} Z_{p, n} \boldsymbol{f}_{n, n} a_{n, 2} a_{q, 2}
$$


3.4.8 Reduktionswege für $a_{p, 1} a_{q, 1} a_{n, 2} \boldsymbol{f}_{n, n} a_{n, 2}$

Beginnend mit $\left(a_{p, 1} a_{q, 1} a_{n, 2} \boldsymbol{f}_{n, n} a_{n, 2}, a_{p, 1} V_{q}\right)$ :

$$
\begin{aligned}
& a_{p, 1} \underline{a_{q, 1} a_{n, 2} \boldsymbol{f}_{n, n} a_{n, 2}} \\
& \mid \stackrel{\widetilde{V}_{q}}{\longrightarrow}-\sum_{j, k=1}^{n} a_{p, 1} Z_{q, j} \boldsymbol{f}_{j, k} a_{k, 1} \\
& -\underline{a_{p, 1} a_{q, 1}}\left(Q+\sum_{\substack{j, k=1 \\
j, k) \neq(n, n)}}^{n} a_{j, 2} \boldsymbol{f}_{j, k} a_{k, 2}\right) \\
& \mid \stackrel{\widetilde{Z}_{p, q}}{\longrightarrow}-\sum_{j, k=1}^{n} a_{p, 1} Z_{q, j} \boldsymbol{f}_{j, k} a_{k, 1} \\
& -Z_{p, q}\left(Q+\sum_{\substack{j, k=1 \\
(j, k) \neq(n, n)}}^{n} a_{j, 2} \boldsymbol{f}_{j, k} a_{k, 2}\right) \\
& =\sum_{j, k=1}^{n}\left(\underline{a_{p, 1} a_{q, 2} a_{j, 2}}-Z_{p, q, j}\right) \boldsymbol{f}_{j, k} a_{k, 1} \\
& -Z_{p, q}\left(Q+\sum_{\substack{j, k=1 \\
(j, k) \neq(n, n)}}^{n} a_{j, 2} \boldsymbol{f}_{j, k} a_{k, 2}\right) \\
& \stackrel{\widetilde{Z}_{p, q, j}}{\longrightarrow} \sum_{j, k=1}^{n}\left(Z_{p, q, j}-Z_{p, q} a_{j, 1}-Z_{p, q, j}\right) \boldsymbol{f}_{j, k} a_{k, 1} \\
& -Z_{p, q}\left(Q+\sum_{\substack{j, k=1 \\
(j, k) \neq(n, n)}}^{n} a_{j, 2} \boldsymbol{f}_{j, k} a_{k, 2}\right) \\
& =Z_{p, q}\left(\underline{a_{n, 1} \boldsymbol{f}_{n, n} a_{n, 1}}+\sum_{\substack{j, k=1 \\
(j, k) \neq(n, n)}}^{n} a_{j, 1} \boldsymbol{f}_{j, k} a_{k, 1}\right. \\
& \left.+Q+\sum_{\substack{j, k=1 \\
j, k) \neq(n, n)}}^{n} a_{j, 2} \boldsymbol{f}_{j, k} a_{k, 2}\right)
\end{aligned}
$$




$$
\begin{aligned}
\stackrel{\widetilde{V}}{\longrightarrow} & Z_{p, q}\left(-Q-\sum_{j, k=1}^{n} a_{j, 2} \boldsymbol{f}_{j, k} a_{k, 2}-\sum_{\substack{j, k=1 \\
(j, k) \neq(n, n)}}^{n} a_{j, 1} \boldsymbol{f}_{j, k} a_{k, 1}\right. \\
& \left.+\sum_{\substack{j, k=1 \\
(j, k) \neq(n, n)}}^{n} a_{j, 1} \boldsymbol{f}_{j, k} a_{k, 1}+Q+\sum_{\substack{j, k=1 \\
(j, k) \neq(n, n)}}^{n} a_{j, 2} \boldsymbol{f}_{j, k} a_{k, 2}\right) \\
= & Z_{p, q} a_{n, 2} \boldsymbol{f}_{n, n} a_{n, 2}
\end{aligned}
$$

Beginnend mit $\left(a_{p, 1} a_{q, 1} a_{n, 2} \boldsymbol{f}_{n, n} a_{n, 2}, Z_{p, q} a_{n, 2} \boldsymbol{f}_{n, n} a_{n, 2}\right)$ :

$$
\begin{aligned}
\widetilde{Z}_{p, q} & \stackrel{a_{p, 1} a_{q, 1} a_{n, 2} \boldsymbol{f}_{n, n} a_{n, 2}}{\longrightarrow} Z_{p, q} a_{n, 2} \boldsymbol{f}_{n, n} a_{n, 2}
\end{aligned}
$$


3.4.9 Reduktionswege für $a_{1, p} a_{1,1} a_{n, 2} \boldsymbol{f}_{n, n} a_{n, 2}$

Beginnend mit $\left(a_{1, p} a_{1,1} a_{n, 2} \boldsymbol{f}_{n, n} a_{n, 2}, a_{1, p} V_{1}\right)$ :

$$
\begin{aligned}
& a_{1, p} a_{1,1} a_{n, 2} \boldsymbol{f}_{n, n} a_{n, 2} \\
& \stackrel{\widetilde{V}_{1}}{\longrightarrow}-\sum_{j, k=1}^{n} a_{1, p} Z_{1, j} \boldsymbol{f}_{j, k} a_{k, 1} \\
& -\underline{a_{1, p} a_{1,1}}\left(Q+\sum_{\substack{j, k=1 \\
(j, k) \neq(n, n)}}^{n} a_{j, 2} \boldsymbol{f}_{j, k} a_{k, 2}\right) \\
& \stackrel{\widetilde{S}_{p, 1}}{\longrightarrow} \sum_{j, k=1}^{n} \sum_{l=2}^{n} \underline{a_{1, p} a_{1, l}} a_{j, l} \boldsymbol{f}_{j, k} a_{k, 1}-\sum_{j, k=1}^{n} a_{1, p} \delta_{1, j} \boldsymbol{f}_{j, k} a_{k, 1} \\
& -S_{p, 1}\left(Q+\sum_{\substack{j, k=1 \\
(j, k) \neq(n, n)}}^{n} a_{j, 2} \boldsymbol{f}_{j, k} a_{k, 2}\right) \\
& \stackrel{\widetilde{S}_{p, l}}{\longrightarrow} \sum_{j, k=1}^{n} \sum_{l=2}^{n} S_{p, 1} a_{j, l} \boldsymbol{f}_{j, k} a_{k, 1}-\sum_{j, k=1}^{n} a_{1, p} \delta_{1, j} \boldsymbol{f}_{j, k} a_{k, 1} \\
& -S_{p, 1}\left(Q+\sum_{\substack{j, k=1 \\
j, k) \neq(n, n)}}^{n} a_{j, 2} \boldsymbol{f}_{j, k} a_{k, 2}\right) \\
& \stackrel{\text { 2.2.1 }}{=} \sum_{j, k=1}^{n}\left(\sum_{l=2}^{n} a_{l, p} Z_{l, j}+\left(\delta_{j, 1}-\delta_{1, p}\right) a_{j, p}\right) \boldsymbol{f}_{j, k} a_{k, 1} \\
& -\sum_{k=1}^{n} a_{1, p} \boldsymbol{f}_{1, k} a_{k, 1}-S_{p, 1}\left(Q+\sum_{\substack{j, k=1 \\
(j, k) \neq(n, n)}}^{n} a_{j, 2} \boldsymbol{f}_{j, k} a_{k, 2}\right) \\
& =\sum_{j, k=1}^{n} \sum_{l=2}^{n} a_{l, p} Z_{l, j} \boldsymbol{f}_{j, k} a_{k, 1}-\sum_{\substack{j, k=1 \\
(j, k) \neq(n, n)}}^{n} \delta_{1, p} a_{j, 1} \boldsymbol{f}_{j, k} a_{k, 1} \\
& -\delta_{1, p} a_{n, 1} \boldsymbol{f}_{n, n} a_{n, 1} \\
& -S_{p, 1}\left(Q+\sum_{\substack{j, k=1 \\
j, k \neq \neq(n, n)}}^{n} a_{j, 2} \boldsymbol{f}_{j, k} a_{k, 2}\right)
\end{aligned}
$$




$$
\begin{aligned}
& \stackrel{\widetilde{V}}{\longrightarrow} \sum_{j, k=1}^{n} \sum_{l=2}^{n} a_{l, p} Z_{l, j} \boldsymbol{f}_{j, k} a_{k, 1}-\sum_{\substack{j, k=1 \\
j, k) \neq(n, n)}}^{n} \delta_{1, p} a_{j, 1} \boldsymbol{f}_{j, k} a_{k, 1} \\
& +\delta_{1, p}\left(Q+\sum_{j, k=1}^{n} a_{j, 2} \boldsymbol{f}_{j, k} a_{k, 2}+\sum_{\substack{j, k=1 \\
j, k) \neq(n, n)}}^{n} a_{j, 1} \boldsymbol{f}_{j, k} a_{k, 1}\right) \\
& -S_{p, 1}\left(Q+\sum_{\substack{j, k=1 \\
(j, k) \neq(n, n)}}^{n} a_{j, 2} \boldsymbol{f}_{j, k} a_{k, 2}\right) \\
& =\sum_{j, k=1}^{n} \sum_{l=2}^{n} a_{l, p} Z_{l, j} \boldsymbol{f}_{j, k} a_{k, 1}+\delta_{1, p}\left(Q+\sum_{j, k=1}^{n} a_{j, 2} \boldsymbol{f}_{j, k} a_{k, 2}\right) \\
& -S_{p, 1}\left(Q+\sum_{\substack{j, k=1 \\
j, k) \neq(n, n)}}^{n} a_{j, 2} \boldsymbol{f}_{j, k} a_{k, 2}\right)
\end{aligned}
$$

Beginnend mit $\left(a_{1, p} a_{1,1} a_{n, 2} \boldsymbol{f}_{n, n} a_{n, 2}, S_{p, 1} a_{n, 2} \boldsymbol{f}_{n, n} a_{n, 2}\right)$ :

$$
\begin{aligned}
& \underline{a_{1, p} a_{1,1}} a_{n, 2} \boldsymbol{f}_{n, n} a_{n, 2} \\
& \mid \stackrel{\widetilde{S}_{p, 1}}{\longrightarrow}-\sum_{l=2}^{n} a_{l, p} \underline{a_{l, 1} a_{n, 2} \boldsymbol{f}_{n, n} a_{n, 2}}+\delta_{p, 1} a_{n, 2} \boldsymbol{f}_{n, n} a_{n, 2} \\
& \stackrel{\widetilde{V}_{l}}{\longrightarrow}+\sum_{l=2}^{n} a_{l, p} \sum_{j, k=1}^{n} Z_{l, j} \boldsymbol{f}_{j, k} a_{k, 1} \\
& +\sum_{l=2}^{n} a_{l, p} a_{l, 1}\left(Q+\sum_{\substack{j, k=1 \\
(j, k) \neq(n, n)}}^{n} a_{j, 2} \boldsymbol{f}_{j, k} a_{k, 2}\right) \\
& +\delta_{p, 1} a_{n, 2} \boldsymbol{f}_{n, n} a_{n, 2} \\
& =+\sum_{l=2}^{n} a_{l, p} \sum_{j, k=1}^{n} Z_{l, j} \boldsymbol{f}_{j, k} a_{k, 1} \\
& -\left(S_{p, 1}-\delta_{p, 1}\right)\left(Q+\sum_{\substack{j, k=1 \\
j, k) \neq(n, n)}}^{n} a_{j, 2} \boldsymbol{f}_{j, k} a_{k, 2}\right) \\
& +\delta_{p, 1} a_{n, 2} \boldsymbol{f}_{n, n} a_{n, 2}
\end{aligned}
$$


3.4.10 Reduktionswege für $a_{1, p} a_{2, q} a_{2,1} a_{n, 2} \boldsymbol{f}_{n, n} a_{n, 2}$

Beginnend mit $\left(a_{1, p} a_{2, q} a_{2,1} a_{n, 2} \boldsymbol{f}_{n, n} a_{n, 2}, a_{1, p} a_{2, q} V_{2}\right)$ :

$$
\begin{aligned}
& a_{1, p} a_{2, q} \underline{a_{2,1} a_{n, 2} \boldsymbol{f}_{n, n} a_{n, 2}} \\
& \stackrel{\widetilde{V}_{2}}{\longrightarrow}-\sum_{j, k=1}^{n} a_{1, p} a_{2, q} Z_{2, j} \boldsymbol{f}_{j, k} a_{k, 1} \\
& -\underline{a_{1, p} a_{2, q} a_{2,1}}\left(Q+\sum_{\substack{j, k=1 \\
(j, k) \neq(n, n)}}^{n} a_{j, 2} \boldsymbol{f}_{j, k} a_{k, 2}\right) \\
& \mid \stackrel{\widetilde{S}_{p, q, 1}}{\longrightarrow} \sum_{j, k=1}^{n} \sum_{i=2}^{n} \underline{a_{1, p} a_{2, q} a_{2, i}} a_{j, i} \boldsymbol{f}_{j, k} a_{k, 1} \\
& -\sum_{j, k=1}^{n} a_{1, p} a_{2, q} \delta_{2, j} \boldsymbol{f}_{j, k} a_{k, 1} \\
& -\left(S_{p, q, 1}-S_{p, q} a_{1,1}\right)\left(Q+\sum_{\substack{j, k=1 \\
(j, k) \neq(n, n)}}^{n} a_{j, 2} \boldsymbol{f}_{j, k} a_{k, 2}\right) \\
& \stackrel{\widetilde{S}_{p, q, i}}{\longrightarrow} \sum_{j, k=1}^{n} \sum_{i=2}^{n} S_{p, q, i} a_{j, i} \boldsymbol{f}_{j, k} a_{k, 1} \\
& -\sum_{j, k=1}^{n} \sum_{i=2}^{n} S_{p, q} a_{1, i} a_{j, i} \boldsymbol{f}_{j, k} a_{k, 1} \\
& -\sum_{k=1}^{n} a_{1, p} a_{2, q} \boldsymbol{f}_{2, k} a_{k, 1} \\
& -\left(S_{p, q, 1}-S_{p, q} a_{1,1}\right)\left(Q+\sum_{\substack{j, k=1 \\
j, k \neq \neq(n, n)}}^{n} a_{j, 2} \boldsymbol{f}_{j, k} a_{k, 2}\right)
\end{aligned}
$$




$$
\begin{aligned}
& =\sum_{j, k=1}^{n} \sum_{i=2}^{n} S_{p, q, i} a_{j, i} \boldsymbol{f}_{j, k} a_{k, 1} \\
& +\sum_{j, k=1}^{n} S_{p, q} Z_{1, j} \boldsymbol{f}_{j, k} a_{k, 1}-\sum_{j, k=1}^{n} S_{p, q} \delta_{1, j} \boldsymbol{f}_{j, k} a_{k, 1} \\
& -\sum_{k=1}^{n} a_{1, p} a_{2, q} \boldsymbol{f}_{2, k} a_{k, 1} \\
& -\left(S_{p, q, 1}-S_{p, q} a_{1,1}\right)\left(Q+\sum_{\substack{j, k=1 \\
j, k \neq \neq(n, n)}}^{n} a_{j, 2} \boldsymbol{f}_{j, k} a_{k, 2}\right) \\
& \stackrel{2.2 .2}{=} \sum_{j, k=1}^{n}\left(\sum_{i=3}^{n} a_{1, p} a_{i, q} Z_{i, j}+\delta_{j, 1} \underline{a_{1, p} a_{1, q}}\right. \\
& \left.+\delta_{j, 2} a_{1, p} a_{2, q}-\delta_{q, 1} a_{1, p} a_{j, 1}\right) \boldsymbol{f}_{j, k} a_{k, 1} \\
& +\sum_{j, k=1}^{n} S_{p, q} Z_{1, j} \boldsymbol{f}_{j, k} a_{k, 1}-\sum_{k=1}^{n} S_{p, q} \boldsymbol{f}_{1, k} a_{k, 1} \\
& -\sum_{k=1}^{n} a_{1, p} a_{2, q} \boldsymbol{f}_{2, k} a_{k, 1} \\
& -\left(S_{p, q, 1}-S_{p, q} a_{1,1}\right)\left(Q+\sum_{\substack{j, k=1 \\
j, k) \neq(n, n)}}^{n} a_{j, 2} \boldsymbol{f}_{j, k} a_{k, 2}\right) \\
& \mid \stackrel{\widetilde{S}_{p, q}}{\longrightarrow} \sum_{j, k=1}^{n} \sum_{i=3}^{n} a_{1, p} a_{i, q} Z_{i, j} \boldsymbol{f}_{j, k} a_{k, 1}+\sum_{k=1}^{n} S_{p, q} \boldsymbol{f}_{1, k} a_{k, 1} \\
& -\delta_{q, 1} a_{1, p}\left(\sum_{\substack{j, k=1 \\
(j, k) \neq(n, n)}}^{n} a_{j, 1} \boldsymbol{f}_{j, k} a_{k, 1}+\underline{a_{n, 1} \boldsymbol{f}_{n, n} a_{n, 1}}\right) \\
& +\sum_{j, k=1}^{n} S_{p, q} Z_{1, j} \boldsymbol{f}_{j, k} a_{k, 1}-\sum_{k=1}^{n} S_{p, q} \boldsymbol{f}_{1, k} a_{k, 1} \\
& -\left(S_{p, q, 1}-S_{p, q} a_{1,1}\right)\left(Q+\sum_{\substack{j, k=1 \\
j, k) \neq(n, n)}}^{n} a_{j, 2} \boldsymbol{f}_{j, k} a_{k, 2}\right)
\end{aligned}
$$




$$
\begin{aligned}
& \stackrel{\widetilde{V}}{\longrightarrow} \sum_{j, k=1}^{n} \sum_{i=3}^{n} a_{1, p} a_{i, q} Z_{i, j} \boldsymbol{f}_{j, k} a_{k, 1} \\
& -\delta_{q, 1} a_{1, p}\left(\sum_{\substack{j, k=1 \\
(j, k) \neq(n, n)}}^{n} a_{j, 1} \boldsymbol{f}_{j, k} a_{k, 1}-Q-\sum_{j, k=1}^{n} a_{j, 2} \boldsymbol{f}_{j, k} a_{k, 2}\right. \\
& \left.-\sum_{\substack{j, k=1 \\
(j, k) \neq(n, n)}}^{n} a_{j, 1} \boldsymbol{f}_{j, k} a_{k, 1}\right) \\
& +\sum_{j, k=1}^{n} S_{p, q} Z_{1, j} \boldsymbol{f}_{j, k} a_{k, 1} \\
& -\left(S_{p, q, 1}-S_{p, q} a_{1,1}\right)\left(Q+\sum_{\substack{j, k=1 \\
(j, k) \neq(n, n)}}^{n} a_{j, 2} \boldsymbol{f}_{j, k} a_{k, 2}\right) \\
& =\sum_{j, k=1}^{n} \sum_{i=3}^{n} a_{1, p} a_{i, q} Z_{i, j} \boldsymbol{f}_{j, k} a_{k, 1}+\sum_{j, k=1}^{n} S_{p, q} Z_{1, j} \boldsymbol{f}_{j, k} a_{k, 1} \\
& +\delta_{q, 1} a_{1, p}\left(Q+\sum_{j, k=1}^{n} a_{j, 2} \boldsymbol{f}_{j, k} a_{k, 2}\right) \\
& -\left(S_{p, q, 1}-S_{p, q} a_{1,1}\right)\left(Q+\sum_{\substack{j, k=1 \\
(j, k) \neq(n, n)}}^{n} a_{j, 2} \boldsymbol{f}_{j, k} a_{k, 2}\right)
\end{aligned}
$$


Beginnend mit $\left(a_{1, p} a_{2, q} a_{2,1} a_{n, 2} \boldsymbol{f}_{n, n} a_{n, 2},\left(S_{p, q, 1}-S_{p, q} a_{1,1}\right) a_{n, 2} \boldsymbol{f}_{n, n} a_{n, 2}\right)$ :

$$
\begin{aligned}
& \underline{a_{1, p} a_{2, q} a_{2,1}} a_{n, 2} \boldsymbol{f}_{n, n} a_{n, 2} \\
& \mid \stackrel{\widetilde{S}_{p, q, 1}}{\longrightarrow}-\sum_{i=3}^{n} a_{1, p} a_{i, q} \underline{a_{i, 1} a_{n, 2} \boldsymbol{f}_{n, n} a_{n, 2}}+\delta_{q, 1} a_{1, p} a_{n, 2} \boldsymbol{f}_{n, n} a_{n, 2} \\
& -S_{p, q} a_{1,1} a_{n, 2} \boldsymbol{f}_{n, n} a_{n, 2} \\
& \stackrel{\tilde{V}_{i}, \widetilde{V}_{1}}{\longrightarrow} \sum_{j, k=1}^{n} \sum_{i=3}^{n} a_{1, p} a_{i, q} Z_{i, j} \boldsymbol{f}_{j, k} a_{k, 1} \\
& +\sum_{i=3}^{n} a_{1, p} a_{i, q} a_{i, 1}\left(Q+\sum_{\substack{j, k=1 \\
j, k) \neq(n, n)}}^{n} a_{j, 2} \boldsymbol{f}_{j, k} a_{k, 2}\right) \\
& +\delta_{q, 1} a_{1, p} a_{n, 2} \boldsymbol{f}_{n, n} a_{n, 2} \\
& +\sum_{j, k=1}^{n} S_{p, q} Z_{1, j} \boldsymbol{f}_{j, k} a_{k, 1} \\
& +S_{p, q} a_{1,1}\left(Q+\sum_{\substack{j, k=1 \\
(j, k) \neq(n, n)}}^{n} a_{j, 2} \boldsymbol{f}_{j, k} a_{k, 2}\right) \\
& =\sum_{j, k=1}^{n} \sum_{i=3}^{n} a_{1, p} a_{i, q} Z_{i, j} \boldsymbol{f}_{j, k} a_{k, 1}+\sum_{j, k=1}^{n} S_{p, q} Z_{1, j} \boldsymbol{f}_{j, k} a_{k, 1} \\
& +\delta_{q, 1} a_{1, p}\left(Q+\sum_{j, k=1}^{n} a_{j, 2} \boldsymbol{f}_{j, k} a_{k, 2}\right) \\
& +\left(S_{p, q} a_{1,1}-S_{p, q, 1}\right)\left(Q+\sum_{\substack{j, k=1 \\
(j, k) \neq(n, n)}}^{n} a_{j, 2} \boldsymbol{f}_{j, k} a_{k, 2}\right)
\end{aligned}
$$


3.4.11 Reduktionswege für $a_{n, 1} \boldsymbol{f}_{n, n} a_{n, 2} a_{1,2} a_{1, q}$

Beginnend mit $\left(a_{n, 1} \boldsymbol{f}_{n, n} a_{n, 2} a_{1,2} a_{1, q}, a_{n, 1} \boldsymbol{f}_{n, n} a_{n, 2}\left(S_{2, q}\right)\right)$ :

$$
\begin{aligned}
& a_{n, 1} \boldsymbol{f}_{n, n} a_{n, 2} a_{1,2} a_{1, q} \\
& \mid \stackrel{\widetilde{S}_{2, q}}{\longrightarrow}-a_{n, 1} \boldsymbol{f}_{n, n} a_{n, 2}\left(\sum_{i=2}^{n} a_{i, 2} a_{i, q}-\delta_{2, q}\right) \\
& =-\sum_{i=2}^{n} \underline{a_{n, 1} \boldsymbol{f}_{n, n} a_{n, 2} a_{i, 2}} a_{i, q}+a_{n, 1} \boldsymbol{f}_{n, n} a_{n, 2} \delta_{2, q} \\
& \stackrel{\widetilde{W}_{i}}{\longrightarrow}-\sum_{i=2}^{n}\left(-a_{n, 1} \boldsymbol{f}_{n, n}\left(\sum_{l=3}^{n} a_{n, l} a_{i, l}-\delta_{n, i}\right)\right. \\
& \left.+\sum_{\substack{j, k=1 \\
(j, k) \neq(n, n)}}^{n} a_{j, 1} \boldsymbol{f}_{j, k} Z_{k, i}+\left(Q+\sum_{j, k=1}^{n} a_{j, 2} \boldsymbol{f}_{j, k} a_{k, 2}\right) a_{i, 1}\right) a_{i, q} \\
& +a_{n, 1} \boldsymbol{f}_{n, n} a_{n, 2} \delta_{2, q} \\
& =\sum_{i=2}^{n} a_{n, 1} \boldsymbol{f}_{n, n}\left(\sum_{l=3}^{n} a_{n, l} a_{i, l}\right) a_{i, q}-\sum_{i=2}^{n} a_{n, 1} \boldsymbol{f}_{n, n}\left(\delta_{n, i}\right) a_{i, q} \\
& \text { - } \sum_{\substack{j, k=1 \\
(j, k) \neq(n, n)}}^{n} a_{j, 1} \boldsymbol{f}_{j, k} \sum_{\underline{i=2}}^{n} Z_{k, i} a_{i, q} \\
& -\left(Q+\sum_{j, k=1}^{n} a_{j, 2} \boldsymbol{f}_{j, k} a_{k, 2}\right) \sum_{i=2}^{n} a_{i, 1} a_{i, q}+a_{n, 1} \boldsymbol{f}_{n, n} a_{n, 2} \delta_{2, q} \\
& \stackrel{2.2 .2}{=} \sum_{l=3}^{n} a_{n, 1} \boldsymbol{f}_{n, n} a_{n, l} \sum_{i=2}^{n} a_{i, l} a_{i, q}-a_{n, 1} \boldsymbol{f}_{n, n} a_{n, q} \\
& -\sum_{\substack{j, k=1 \\
(j, k) \neq(n, n)}}^{n} a_{j, 1} \boldsymbol{f}_{j, k}\left(\sum_{i=2}^{n} a_{k, i} S_{i, q}+\left(\delta_{1, q}-\delta_{k, 1}\right) a_{k, q}\right) \\
& +\left(Q+\sum_{j, k=1}^{n} a_{j, 2} \boldsymbol{f}_{j, k} a_{k, 2}\right)\left(S_{1, q}-\delta_{1, q}\right)+a_{n, 1} \boldsymbol{f}_{n, n} a_{n, 2} \delta_{2, q}
\end{aligned}
$$




$$
\begin{aligned}
& -\sum_{l=3}^{n} a_{n, 1} \boldsymbol{f}_{n, n} a_{n, l}\left(S_{l, q}-\delta_{l, q}\right)-a_{n, 1} \boldsymbol{f}_{n, n} a_{n, q} \\
& -\sum_{\substack{j, k=1 \\
(j, k) \neq(n, n)}}^{n} a_{j, 1} \boldsymbol{f}_{j, k}\left(\sum_{i=2}^{n} a_{k, i} S_{i, q}+\left(\delta_{1, q}-\delta_{k, 1}\right) a_{k, q}\right) \\
& +\left(Q+\sum_{j, k=1}^{n} a_{j, 2} \boldsymbol{f}_{j, k} a_{k, 2}\right)\left(S_{1, q}-\delta_{1, q}\right)+a_{n, 1} \boldsymbol{f}_{n, n} a_{n, 2} \delta_{2, q} \\
& =-\sum_{l=3}^{n} a_{n, 1} \boldsymbol{f}_{n, n} a_{n, l}\left(S_{l, q}\right) \\
& -\sum_{\substack{j, k=1 \\
(j, k) \neq(n, n)}}^{n} a_{j, 1} \boldsymbol{f}_{j, k}\left(\sum_{i=2}^{n} a_{k, i} S_{i, q}-\delta_{k, 1} a_{k, q}\right) \\
& +\left(Q+\sum_{j, k=1}^{n} a_{j, 2} \boldsymbol{f}_{j, k} a_{k, 2}\right)\left(S_{1, q}\right) \\
& +\sum_{l=3}^{n} a_{n, 1} \boldsymbol{f}_{n, n} a_{n, l}\left(\delta_{l, q}\right)-a_{n, 1} \boldsymbol{f}_{n, n} a_{n, q} \\
& -\sum_{\substack{j, k=1 \\
(j, k) \neq(n, n)}}^{n} a_{j, 1} \boldsymbol{f}_{j, k}\left(\delta_{1, q} a_{k, q}\right) \\
& +\left(Q+\sum_{j, k=1}^{n} a_{j, 2} \boldsymbol{f}_{j, k} a_{k, 2}\right)\left(-\delta_{1, q}\right)+a_{n, 1} \boldsymbol{f}_{n, n} a_{n, 2} \delta_{2, q} \\
& =-\sum_{l=3}^{n} a_{n, 1} \boldsymbol{f}_{n, n} a_{n, l} S_{l, q} \\
& -\sum_{\substack{j, k=1 \\
(j, k) \neq(n, n)}}^{n} a_{j, 1} \boldsymbol{f}_{j, k}\left(\sum_{i=2}^{n} a_{k, i} S_{i, q}-\delta_{k, 1} a_{k, q}\right) \\
& +\left(Q+\sum_{j, k=1}^{n} a_{j, 2} \boldsymbol{f}_{j, k} a_{k, 2}\right)\left(S_{1, q}\right) \\
& -\delta_{1, q}\left(\underline{a_{n, 1} \boldsymbol{f}_{n, n} a_{n, 1}}\right. \\
& \left.+\sum_{\substack{j, k=1 \\
(j, k) \neq(n, n)}}^{n} a_{j, 1} \boldsymbol{f}_{j, k} a_{k, 1}+\left(Q+\sum_{j, k=1}^{n} a_{j, 2} \boldsymbol{f}_{j, k} a_{k, 2}\right)\right)
\end{aligned}
$$




$$
\begin{aligned}
& \stackrel{\widetilde{V}}{\longrightarrow}-\sum_{l=3}^{n} a_{n, 1} \boldsymbol{f}_{n, n} a_{n, l} S_{l, q} \\
& -\sum_{\substack{j, k=1 \\
(j, k) \neq(n, n)}}^{n} a_{j, 1} \boldsymbol{f}_{j, k}\left(\sum_{i=2}^{n} a_{k, i} S_{i, q}-\delta_{k, 1} a_{k, q}\right) \\
& +\left(Q+\sum_{j, k=1}^{n} a_{j, 2} \boldsymbol{f}_{j, k} a_{k, 2}\right)\left(S_{1, q}\right) \\
& -\delta_{1, q}\left(-\left(Q+\sum_{j, k=1}^{n} a_{j, 2} \boldsymbol{f}_{j, k} a_{k, 2}+\sum_{\substack{j, k=1 \\
(j, k) \neq(n, n)}}^{n} a_{j, 1} \boldsymbol{f}_{j, k} a_{k, 1}\right)\right. \\
& \left.+\sum_{\substack{j, k=1 \\
(j, k) \neq(n, n)}}^{n} a_{j, 1} \boldsymbol{f}_{j, k} a_{k, 1}+\left(Q+\sum_{j, k=1}^{n} a_{j, 2} \boldsymbol{f}_{j, k} a_{k, 2}\right)\right) \\
& =-\sum_{l=3}^{n} a_{n, 1} \boldsymbol{f}_{n, n} a_{n, l} S_{l, q} \\
& -\sum_{\substack{j, k=1 \\
(j, k) \neq(n, n)}}^{n} a_{j, 1} \boldsymbol{f}_{j, k}\left(\sum_{i=2}^{n} a_{k, i} S_{i, q}-\delta_{k, 1} a_{k, q}\right) \\
& +\left(Q+\sum_{j, k=1}^{n} a_{j, 2} \boldsymbol{f}_{j, k} a_{k, 2}\right)\left(S_{1, q}\right)
\end{aligned}
$$


Beginnend mit $\left(a_{n, 1} \boldsymbol{f}_{n, n} a_{n, 2} a_{1,2} a_{1, q}, W_{1} a_{1, q}\right)$ :

$$
\begin{aligned}
& \mid \stackrel{\widetilde{W}_{1}}{\longrightarrow}-a_{n, 1} \boldsymbol{f}_{n, n}\left(\sum_{l=3}^{n} a_{n, l} a_{1, l}-\delta_{n, 1}\right) a_{1, q} \\
& +\sum_{\substack{j, k=1 \\
(j, k) \neq(n, n)}}^{n} a_{j, 1} \boldsymbol{f}_{j, k} Z_{k, 1} a_{1, q} \\
& +\left(Q+\sum_{j, k=1}^{n} a_{j, 2} \boldsymbol{f}_{j, k} a_{k, 2}\right) a_{1,1} a_{1, q} \\
& =-a_{n, 1} \boldsymbol{f}_{n, n} \sum_{l=3}^{n} a_{n, l} \underline{a_{1, l} a_{1, q}} \\
& -\sum_{\substack{j, k=1 \\
(j, k) \neq(n, n)}}^{n} a_{j, 1} \boldsymbol{f}_{j, k}\left(\sum_{i=2}^{n} a_{k, i} \underline{a_{1, i} a_{1, q}}-\delta_{k, 1} a_{1, q}\right) \\
& +\left(Q+\sum_{j, k=1}^{n} a_{j, 2} \boldsymbol{f}_{j, k} a_{k, 2}\right) \underline{a_{1,1} a_{1, q}} \\
& \mid \stackrel{\widetilde{S}_{l, q}, \widetilde{S}_{i, q}, \widetilde{S}_{1, q}}{\rightarrow}-a_{n, 1} \boldsymbol{f}_{n, n} \sum_{l=3}^{n} a_{n, l} S_{l, q} \\
& -\sum_{\substack{j, k=1 \\
(j, k) \neq(n, n)}}^{n} a_{j, 1} \boldsymbol{f}_{j, k}\left(\sum_{i=2}^{n} a_{k, i} S_{i, q}-\delta_{k, 1} a_{1, q}\right) \\
& +\left(Q+\sum_{j, k=1}^{n} a_{j, 2} \boldsymbol{f}_{j, k} a_{k, 2}\right) S_{1, q}
\end{aligned}
$$


3.4.12 Reduktionswege für $a_{n, 1} \boldsymbol{f}_{n, n} a_{n, 2} a_{1,2} a_{2, q} a_{2, r}$

Um die Lesbarkeit zu erhöhen setzen wir:

$$
\begin{aligned}
\boldsymbol{X}:= & -\sum_{l=3}^{n} a_{n, 1} \boldsymbol{f}_{n, n} a_{n, l}\left(S_{l, q, r}-S_{1, q} a_{1, r}\right) \\
& -\sum_{\substack{j, k=1 \\
(j, k) \neq(n, n)}}^{n} a_{j, 1} \boldsymbol{f}_{j, k} Z_{k, 1}\left(\sum_{i=3}^{n} a_{i, q} a_{i, r}-\delta_{q, r}\right) \\
& +\left(Q+\sum_{j, k=1}^{n} a_{j, 2} \boldsymbol{f}_{j, k} a_{k, 2}\right)\left(S_{1, q, r}-S_{1, q} a_{1, r}\right) .
\end{aligned}
$$

Beginnend mit $\left(a_{n, 1} \boldsymbol{f}_{n, n} a_{n, 2} a_{1,2} a_{2, q} a_{2, r}, a_{n, 1} \boldsymbol{f}_{n, n} a_{n, 2}\left(S_{2, q, r}-S_{2, q} a_{1, r}\right)\right)$ :

$$
\begin{aligned}
& a_{n, 1} \boldsymbol{f}_{n, n} a_{n, 2} \underline{a_{1,2} a_{2, q} a_{2, r}} \\
\hline \widetilde{S}_{2, q, r} & -a_{n, 1} \boldsymbol{f}_{n, n} a_{n, 2} a_{1,2}\left(\sum_{i=3}^{n} a_{i, q} a_{i, r}-\delta_{q, r}\right) \\
& +\sum_{i=2}^{n} \frac{a_{n, 1} \boldsymbol{f}_{n, n} a_{n, 2} a_{i, 2} a_{i, q} a_{1, r}}{\longrightarrow} \\
& -a_{n, 1} \boldsymbol{f}_{n, n} a_{n, 2} \delta_{2, q} a_{1, r} \\
\widetilde{W}_{1}, \widetilde{W}_{i} & \left(\begin{array}{l}
a_{n, 1} \boldsymbol{f}_{n, n}\left(\sum_{l=3}^{n} a_{n, l} a_{1, l}-\delta_{n, 1}\right) \\
\longrightarrow
\end{array}\right. \\
& -\left(\sum_{\substack{j, k=1 \\
(j, k) \neq(n, n)}}^{n} a_{j, 1} \boldsymbol{f}_{j, k} Z_{k, 1}\right. \\
& -\left(\begin{array}{l}
\left.Q+\sum_{j, k=1}^{n} a_{j, 2} \boldsymbol{f}_{j, k} a_{k, 2}\right) \\
Q
\end{array}\right)\left(\sum_{i, 1}^{n} a_{i, q} a_{i, r}-\delta_{q, r}\right)
\end{aligned}
$$




$$
\begin{aligned}
& -\sum_{i=2}^{n} a_{n, 1} \boldsymbol{f}_{n, n}\left(\sum_{l=3}^{n} a_{n, l} a_{i, l}-\delta_{n, i}\right) a_{i, q} a_{1, r} \\
& +\sum_{i=2}^{n} \sum_{\substack{j, k=1 \\
(j, k) \neq(n, n)}}^{n} a_{j, 1} \boldsymbol{f}_{j, k} Z_{k, i} a_{i, q} a_{1, r} \\
& +\sum_{i=2}^{n}\left(Q+\sum_{j, k=1}^{n} a_{j, 2} \boldsymbol{f}_{j, k} a_{k, 2}\right) a_{i, 1} a_{i, q} a_{1, r} \\
& -a_{n, 1} \boldsymbol{f}_{n, n} a_{n, 2} \delta_{2, q} a_{1, r} \\
& =-\sum_{l=3}^{n} a_{n, 1} \boldsymbol{f}_{n, n} a_{n, l} S_{l, q, r} \\
& \text { - } \sum_{\substack{j, k=1 \\
(j, k) \neq(n, n)}}^{n} a_{j, 1} \boldsymbol{f}_{j, k} Z_{k, 1}\left(\sum_{i=3}^{n} a_{i, q} a_{i, r}-\delta_{q, r}\right) \\
& +\left(Q+\sum_{j, k=1}^{n} a_{j, 2} \boldsymbol{f}_{j, k} a_{k, 2}\right) S_{1, q, r} \\
& -a_{n, 1} \boldsymbol{f}_{n, n} \sum_{l=3}^{n} a_{n, l}\left(\sum_{i=2}^{n} a_{i, l} a_{i, q}\right) a_{1, r} \\
& +\sum_{i=2}^{n} a_{n, 1} \boldsymbol{f}_{n, n}\left(\delta_{n, i}\right) a_{i, q} a_{1, r} \\
& +\sum_{i=2}^{n} \sum_{\substack{j, k=1 \\
j, k) \neq(n, n)}}^{n} a_{j, 1} \boldsymbol{f}_{j, k} Z_{k, i} a_{i, q} a_{1, r} \\
& +\left(Q+\sum_{j, k=1}^{n} a_{j, 2} \boldsymbol{f}_{j, k} a_{k, 2}\right)\left(\sum_{i=2}^{n} a_{i, 1} a_{i, q}\right) a_{1, r} \\
& -a_{n, 1} \boldsymbol{f}_{n, n} a_{n, 2} \delta_{2, q} a_{1, r} \\
& =\boldsymbol{X}-\sum_{l=3}^{n} a_{n, 1} \boldsymbol{f}_{n, n} a_{n, l} \delta_{l, q} a_{1, r}-a_{n, 1} \boldsymbol{f}_{n, n} a_{n, 2} \delta_{2, q} a_{1, r} \\
& +\sum_{i=2}^{n} a_{n, 1} \boldsymbol{f}_{n, n}\left(\delta_{n, i}\right) a_{i, q} a_{1, r} \\
& +\sum_{\substack{j, k=1 \\
(j, k) \neq(n, n)}}^{n} a_{j, 1} \boldsymbol{f}_{j, k} \sum_{\underline{i=2}}^{n} Z_{k, i} a_{i, q} a_{1, r} \\
& +\left(Q+\sum_{j, k=1}^{n} a_{j, 2} \boldsymbol{f}_{j, k} a_{k, 2}\right)\left(\delta_{1, q}\right) a_{1, r}
\end{aligned}
$$




$$
\begin{aligned}
& \stackrel{2.2 .2}{=} \boldsymbol{X}+\delta_{q, 1} a_{n, 1} \boldsymbol{f}_{n, n} a_{n, 1} a_{1, r} \\
& +\sum_{\substack{j, k=1 \\
j, k) \neq(n, n)}}^{n} a_{j, 1} \boldsymbol{f}_{j, k}\left(\sum_{i=2}^{n} a_{k, i} S_{i, q}+\left(\delta_{1, q}-\delta_{k, 1}\right) a_{k, q}\right) a_{1, r} \\
& +\delta_{1, q}\left(Q+\sum_{j, k=1}^{n} a_{j, 2} \boldsymbol{f}_{j, k} a_{k, 2}\right) a_{1, r} \\
& \stackrel{\widetilde{V}}{\longrightarrow} \boldsymbol{X}-\delta_{q, 1}\left(Q+\sum_{j, k=1}^{n} a_{j, 2} \boldsymbol{f}_{j, k} a_{k, 2}\right. \\
& \left.+\sum_{\substack{j, k=1 \\
(j, k) \neq(n, n)}}^{n} a_{j, 1} \boldsymbol{f}_{j, k} a_{k, 1}\right) a_{1, r} \\
& +\sum_{\substack{j, k=1 \\
(j, k) \neq(n, n)}}^{n} a_{j, 1} \boldsymbol{f}_{j, k}\left(\sum_{i=2}^{n} a_{k, i} S_{i, q} a_{1, r}-\delta_{k, 1} \underline{a_{1, q} a_{1, r}}\right) \\
& +\delta_{1, q}\left(\sum_{\substack{j, k=1 \\
(j, k) \neq(n, n)}}^{n} a_{j, 1} \boldsymbol{f}_{j, k} a_{k, 1}\right. \\
& \left.+Q+\sum_{j, k=1}^{n} a_{j, 2} \boldsymbol{f}_{j, k} a_{k, 2}\right) a_{1, r} \\
& \mid \stackrel{\widetilde{Z}_{q, r}}{\longrightarrow} \boldsymbol{X}+\sum_{\substack{j, k=1 \\
(j, k) \neq(n, n)}}^{n} a_{j, 1} \boldsymbol{f}_{j, k}\left(\sum_{i=2}^{n} a_{k, i} S_{i, q} a_{1, r}-\delta_{k, 1} Z_{q, r}\right)
\end{aligned}
$$


Beginnend mit $\left(a_{n, 1} \boldsymbol{f}_{n, n} a_{n, 2} a_{1,2} a_{2, q} a_{2, r},{ }\right)$ :

$$
\begin{aligned}
& a_{n, 1} \boldsymbol{f}_{n, n} a_{n, 2} a_{1,2} a_{2, q} a_{2, r} \\
& \stackrel{\widetilde{W}_{1}}{\longrightarrow}-a_{n, 1} \boldsymbol{f}_{n, n}\left(\sum_{l=3}^{n} a_{n, l} a_{1, l}-\delta_{n, 1}\right) a_{2, q} a_{2, r} \\
& +\sum_{\substack{j, k=1 \\
(j, k) \neq(n, n)}}^{n} a_{j, 1} \boldsymbol{f}_{j, k} Z_{k, 1} a_{2, q} a_{2, r} \\
& +\left(Q+\sum_{j, k=1}^{n} a_{j, 2} \boldsymbol{f}_{j, k} a_{k, 2}\right) \underline{a_{1,1} a_{2, q} a_{2, r}} \\
& \mid \stackrel{\widetilde{S}_{1, q, r}}{\longrightarrow}-a_{n, 1} \boldsymbol{f}_{n, n} \sum_{l=3}^{n} a_{n, l} \underline{a_{1, l} a_{2, q} a_{2, r}} \\
& +\sum_{\substack{j, k=1 \\
(j, k) \neq(n, n)}}^{n} a_{j, 1} \boldsymbol{f}_{j, k} Z_{k, 1} a_{2, q} a_{2, r} \\
& +\left(Q+\sum_{j, k=1}^{n} a_{j, 2} \boldsymbol{f}_{j, k} a_{k, 2}\right)\left(S_{1, q, r}-S_{1, q} a_{1, r}\right) \\
& \mid \stackrel{\widetilde{S}_{l, q, r}}{\longrightarrow}-a_{n, 1} \boldsymbol{f}_{n, n} \sum_{l=3}^{n} a_{n, l}\left(S_{l, q, r}-S_{l, q} a_{1, r}\right) \\
& +\sum_{\substack{j, k=1 \\
(j, k) \neq(n, n)}}^{n} a_{j, 1} \boldsymbol{f}_{j, k} \underline{Z_{k, 1} a_{2, q} a_{2, r}} \\
& +\left(Q+\sum_{j, k=1}^{n} a_{j, 2} \boldsymbol{f}_{j, k} a_{k, 2}\right)\left(S_{1, q, r}-S_{1, q} a_{1, r}\right) \\
& \stackrel{2.2 .9}{=}-a_{n, 1} \boldsymbol{f}_{n, n} \sum_{l=3}^{n} a_{n, l}\left(S_{l, q, r}-S_{l, q} a_{1, r}\right) \\
& +\sum_{\substack{j, k=1 \\
(j, k) \neq(n, n)}}^{n} a_{j, 1} \boldsymbol{f}_{j, k}\left(\sum_{i=2}^{n} \sum_{l=3}^{n} a_{k, i} a_{1, i} a_{l, q} a_{l, r}+Z_{k, 1} \delta_{q, r}\right. \\
& \left.+\sum_{i=2}^{n} a_{k, i} S_{i, q} a_{1, r}-\delta_{k, 1}\left(\delta_{q, r}-a_{2, q} a_{2, r}\right)\right) \\
& +\left(Q+\sum_{j, k=1}^{n} a_{j, 2} \boldsymbol{f}_{j, k} a_{k, 2}\right)\left(S_{1, q, r}-S_{1, q} a_{1, r}\right)
\end{aligned}
$$




$$
\begin{aligned}
& =-a_{n, 1} \boldsymbol{f}_{n, n} \sum_{l=3}^{n} a_{n, l}\left(S_{l, q, r}-S_{l, q} a_{1, r}\right) \\
& +\sum_{\substack{j, k=1 \\
(j, k) \neq(n, n)}}^{n} a_{j, 1} \boldsymbol{f}_{j, k}\left(\sum_{i=2}^{n} \sum_{l=3}^{n} a_{k, i} a_{1, i} a_{l, q} a_{l, r}+Z_{k, 1} \delta_{q, r}\right. \\
& \left.+\sum_{i=2}^{n} a_{k, i} S_{i, q} a_{1, r}-\delta_{k, 1}\left(\delta_{q, r}-a_{2, q} a_{2, r}\right)\right) \\
& +\left(Q+\sum_{j, k=1}^{n} a_{j, 2} \boldsymbol{f}_{j, k} a_{k, 2}\right)\left(S_{1, q, r}-S_{1, q} a_{1, r}\right) \\
& =-a_{n, 1} \boldsymbol{f}_{n, n} \sum_{l=3}^{n} a_{n, l}\left(S_{l, q, r}-S_{l, q} a_{1, r}\right) \\
& +\left(Q+\sum_{j, k=1}^{n} a_{j, 2} \boldsymbol{f}_{j, k} a_{k, 2}\right)\left(S_{1, q, r}-S_{1, q} a_{1, r}\right) \\
& -\sum_{\substack{j, k=1 \\
(j, k) \neq(n, n)}}^{n} a_{j, 1} \boldsymbol{f}_{j, k}\left(\sum_{l=3}^{n}\left(Z_{k, 1}-\delta_{k, 1}\right) a_{l, q} a_{l, r}-Z_{k, 1} \delta_{q, r}\right) \\
& +\sum_{\substack{j, k=1 \\
(j, k) \neq(n, n)}}^{n} a_{j, 1} \boldsymbol{f}_{j, k} \sum_{i=2}^{n} a_{k, i} S_{i, q} a_{1, r} \\
& -\sum_{\substack{j, k=1 \\
(j, k) \neq(n, n)}}^{n} a_{j, 1} \boldsymbol{f}_{j, k} \delta_{k, 1}\left(\delta_{q, r}-a_{2, q} a_{2, r}\right) \\
& =\boldsymbol{X}+\sum_{\substack{j, k=1 \\
(j, k) \neq(n, n)}}^{n} a_{j, 1} \boldsymbol{f}_{j, k} \delta_{k, 1}\left(\sum_{l=3}^{n} a_{l, q} a_{l, r}-\left(\delta_{q, r}-a_{2, q} a_{2, r}\right)\right) \\
& +\sum_{\substack{j, k=1 \\
(j, k) \neq(n, n)}}^{n} a_{j, 1} \boldsymbol{f}_{j, k} \sum_{i=2}^{n} a_{k, i} S_{i, q} a_{1, r} \\
& =\boldsymbol{X}-\sum_{\substack{j, k=1 \\
(j, k) \neq(n, n)}}^{n} a_{j, 1} \boldsymbol{f}_{j, k}\left(\delta_{k, 1} Z_{q, r}-\sum_{i=2}^{n} a_{k, i} S_{i, q} a_{1, r}\right)
\end{aligned}
$$




\section{Kapitel 4}

\section{(Ko)-Homologie}

Wir wollen im Folgenden 1-dimensionale Moduln über der orthogonalen freien Quantengruppe $\mathcal{A}$ betrachten.

Sei $\mathcal{A}(n)$ die in Abschnitt 2.1 definierte freie Quantengruppe. Zur Erinnerung: Wir können die Erzeuger von $\mathcal{A}$ schreiben als $n \times n$-Matrix $A:=\left(a_{p, q}\right)_{p, q=1, \ldots, n}$ und die Relationen als $A A^{t}=A^{t} A=$ id.

Definition 4.0.1 (1-dimensionaler $\mathcal{A}$-Modul $\mathbb{K}_{\Lambda}$ )

Ein $\mathcal{A}$-Modul, zu dem es eine 1-dimensionale Darstellung gibt, die gegeben ist durch einen Algebra-Homomorphismus :

$$
\begin{aligned}
\mathcal{A} & \rightarrow \mathbb{K} \\
a_{j, i} & \mapsto \lambda_{j, i},
\end{aligned}
$$

heißt 1-dimensionaler Modul. Die Modulstruktur ist gegeben durch:

$$
\begin{array}{ccc}
\mathcal{A} \times \mathbb{K} & \rightarrow & \mathbb{K} \\
a_{j, i}, x & \mapsto & \lambda_{j, i} \cdot x .
\end{array}
$$

Diesen Modul bezeichnen wir mit $\mathbb{K}_{\Lambda}$. Da die Multiplikation in $\mathbb{K}$ kommutiert, ist jeder 1-dimensionale Modul immer auch ein Bimodul.

Die Bilder der Erzeuger schreiben wir wieder als Matrix: $\Lambda:=\left(\lambda_{p, q}\right)_{p, q=1, \ldots, n}$. Für diese Matrix geltenähnliche Relationen wie für die Erzeuger-Matrix $A$ :

$$
\Lambda \Lambda^{t}=\Lambda^{t} \Lambda=\mathrm{id}
$$

sie ist also orthogonal.

Umgekehrt entspricht jede orthogonale Matrix $M$ einem 1-dimensionalen $\mathcal{A}$-Modul $\mathbb{K}_{M}$. 


\subsubsection{Spezialfall $n=1$}

Falls $n=1$, dann hat die Algebra $\mathcal{A}(1)$ nur einen Erzeuger $a_{1,1}$ und nur eine Relation $a_{1,1} a_{1,1}=1$. Diese Algebra ist isomorph zu der Algebra $\mathbb{K}[z] /\left(z^{2}-1\right)$.

Wir wollen uns zunächst überlegen, warum diese Algebra halbeinfach ist, falls $\operatorname{char}(\mathbb{K}) \neq$ 2 .

Seien $e_{1}=\frac{z+1}{2}$ und $e_{2}=\frac{z-1}{2}$, dann sind $e_{1}$ und $e_{2}$ orthogonale Idempotenten, so dass $e_{1}+e_{2}=1$.

Insbesondere

$$
\mathcal{A}(1) \cong \mathbb{K}<e_{1}>\oplus \mathbb{K}<e_{2}>.
$$

Wir bemerken weiterhin, dass es zu $\mathcal{A}(1)$ nur zwei eindimensionale Moduln gibt: Einen Modul $\mathbb{K}_{1}$ zu $e_{1}$ und einen Modul $\mathbb{K}_{2}$ zu $e_{2}$. Diese Moduln sind beide projektiv als direkte Summanden des freien Moduls $\mathcal{A}(1)$.

Da sie projektiv sind, verschwinden alle höheren Ext- und Torgruppen zwischen ihnen.

Falls $\operatorname{char}(\mathbb{K})=2$, dann gibt es nur einen eindimensionalen Modul $\mathbb{K}$, der durch folgende eindimensionale Darstellung gegeben ist:

$$
\begin{aligned}
& \phi: \quad \mathbb{K}[z] /\left(z^{2}-1\right) \rightarrow \mathbb{K} \\
& z \quad \mapsto 1 \\
& 1 \longmapsto 1 .
\end{aligned}
$$

Dieser Modul hat eine unendliche freie Auflösung

$$
0 \leftarrow \mathbb{K} \leftarrow \mathcal{A}(1) \leftarrow \cdots \leftarrow \mathcal{A}(1) \leftarrow \cdots,
$$

wobei jedes Differential durch Multiplikation mit $z+1$ gegeben ist.

Für die Tor- und Extgruppen gilt also:

$$
\begin{aligned}
& \operatorname{Ext}^{k}(\mathbb{K}, \mathbb{K}) \cong \mathbb{K} \text { für alle } k \geq 0, \\
& \operatorname{Tor}_{k}(\mathbb{K}, \mathbb{K}) \cong \mathbb{K} \text { für alle } k \geq 0 .
\end{aligned}
$$




\subsubsection{Auflösung für $n \geq 3$}

Als Reduktionssystem $r_{\boldsymbol{A}}$ für die Algebra $\mathcal{A}(n)$ nehmen wir das in Satz 2.1.3 definierte:

$$
\begin{aligned}
\widetilde{Z}_{p, q} & :=\left(a_{p, 1} a_{q, 1},-\sum_{i=2}^{n} a_{p, i} a_{q, i}+\delta_{p, q}\right), \\
\widetilde{S}_{p, q} & :=\left(a_{1, p} a_{1, q},-\sum_{i=2}^{n} a_{i, p} a_{i, q}+\delta_{p, q}\right), \\
\widetilde{Z}_{p, q, r} & :=\left(a_{p, 1} a_{q, 2} a_{r, 2},-a_{p, 1} \sum_{i=3}^{n} a_{q, i} a_{r, i}+a_{p, 1} \delta_{q, r}-\left(-\sum_{i=2}^{n} a_{p, i} a_{q, i}+\delta_{p, q}\right) a_{r, 1}\right), \\
\widetilde{S}_{p, q, r} & :=\left(a_{1, p} a_{2, q} a_{2, r},-a_{1, p} \sum_{i=3}^{n} a_{i, q} a_{i, r}+a_{1, p} \delta_{q, r}-\left(-\sum_{i=2}^{n} a_{i, p} a_{i, q}+\delta_{p, q}\right) a_{1, r}\right) .
\end{aligned}
$$

Alle Moduln in der Bimodulauflösung in Abschnitt 3.1 sind freie $\mathcal{A}$-Moduln. Wenn wir also die Bimodulauflösung von rechts mit $\otimes_{\mathcal{A}} \mathbb{K}_{\lambda}$ multiplizieren, ist die resultierende Sequenz wieder exakt. Mit $\mathcal{A} \otimes_{\mathcal{A}} \mathbb{K}_{\lambda} \cong \mathbb{K}_{\lambda}$ erhalten wir die folgende Sequenz, bzw. die Auflösung von $\mathbb{K}_{\lambda}$ :

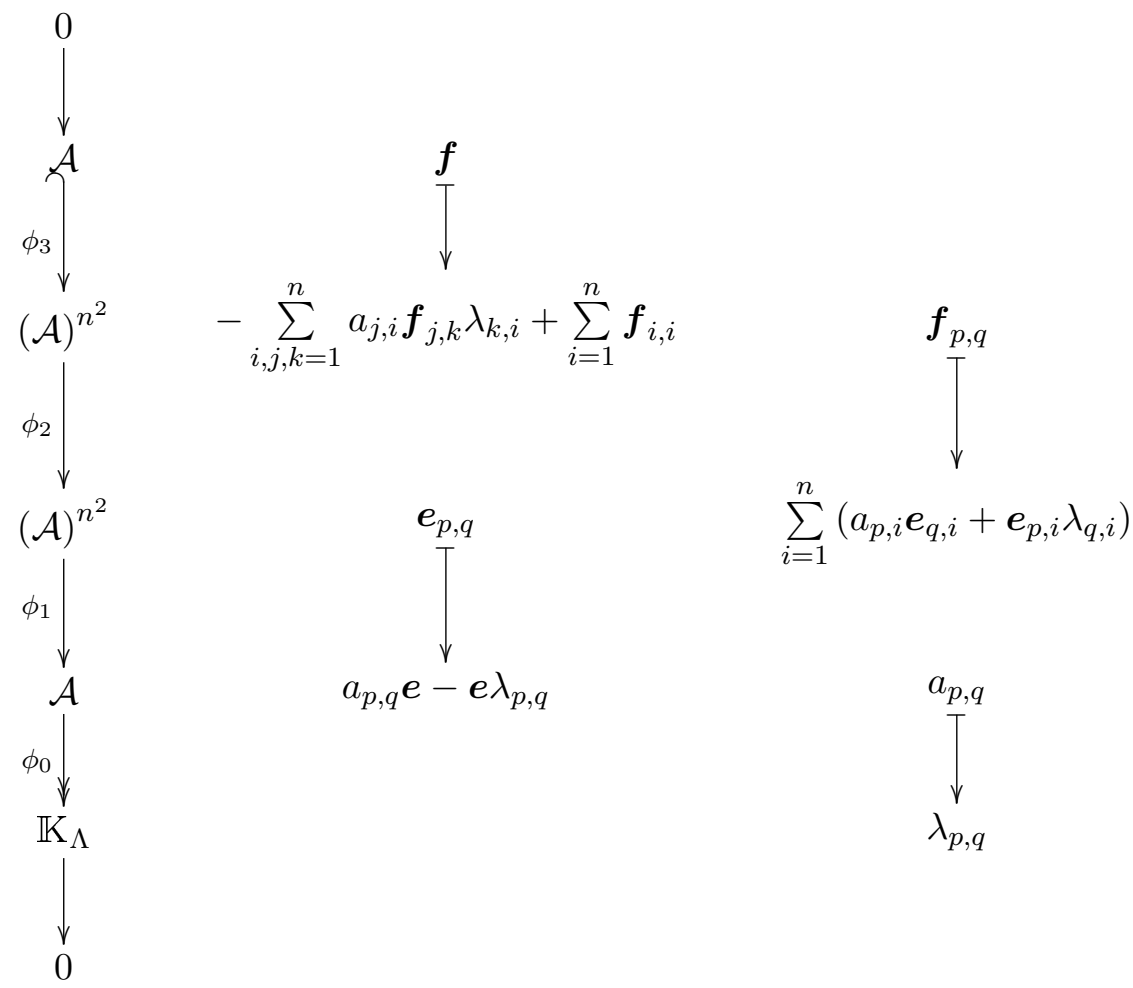

Die fett geschriebenen Buchstaben $\boldsymbol{e}, \boldsymbol{e}_{p, q}, \boldsymbol{f}_{p, q}$ und $\boldsymbol{f}$ stehen für die Erzeuger der Moduln. Die Elemente aus $\mathbb{K}_{\Lambda}$ kommutieren mit denen aus $\mathcal{A}$, daher können wir sie vorziehen.

Zur besseren Übersicht schreiben wir die Auflösung auch in Matrizen-Schreibweise. Mit den Buchstaben $\boldsymbol{E}$ und $\boldsymbol{F}$ bezeichnen wir die Matrizen der entsprechenden Erzeu- 
ger, also $\boldsymbol{E}:=\left(\boldsymbol{e}_{p, q}\right)_{p, q=1, \ldots, n}$ und $\boldsymbol{F}:=\left(\boldsymbol{f}_{p, q}\right)_{p, q=1, \ldots, n}$.

\begin{tabular}{|l|l|}
\hline Koeffizienten vorne & Koeffizienten hinten \\
\hline$\phi_{3}: \boldsymbol{f} \mapsto-\operatorname{tr}\left(\Lambda A^{t} \boldsymbol{F}\right)+\operatorname{tr}(\boldsymbol{F})$ & $\phi_{3}: \boldsymbol{f} \mapsto-\operatorname{tr}\left(A^{t} \boldsymbol{F} \Lambda\right)+\operatorname{tr}(\boldsymbol{F})$ \\
\hline$\phi_{2}: \boldsymbol{F} \mapsto A \boldsymbol{E}^{t}+\left(\Lambda \boldsymbol{E}^{t}\right)^{t}$ & $\phi_{2}: \boldsymbol{F} \mapsto A \boldsymbol{E}^{t}+\boldsymbol{E} \Lambda^{t}$ \\
\hline$\phi_{1}: \boldsymbol{E} \mapsto(A-\Lambda) \boldsymbol{e}$ & $\phi_{1}: \boldsymbol{E} \mapsto A \boldsymbol{e}-\boldsymbol{e} \Lambda$ \\
\hline$\phi_{0}: a_{i, j} \boldsymbol{e} \mapsto \lambda_{i, j}$ & $\phi_{0}: a_{i, j} \boldsymbol{e} \mapsto \lambda_{i, j}$ \\
\hline
\end{tabular}

Hierbei beachten wir, dass das Transponieren nicht wie üblich mit der Multiplikation verträglich ist:

$$
(A B)_{p, q}^{t}=\sum_{i=1}^{n} a_{q, i} b_{i, p} \text { aber }\left(B^{t} A^{t}\right)_{p, q}=\sum_{i=1}^{n} b_{i, p} a_{q, i} .
$$

Im Spezialfall $\Lambda=$ id erhalten wir, dass die in [TC06] vorgestellte Sequenz exakt ist:

$$
\begin{aligned}
& 0 \longleftarrow \mathbb{K}_{\mathrm{id}} \ll \quad \mathcal{A} \leftarrow \mathcal{A}^{n^{2} \leftarrow} \quad \mathcal{A}^{n^{2} \leftarrow} \quad \mathcal{A}_{\mathcal{A}}<0 \\
& \delta_{p, q} \longleftarrow a_{p, q} \quad \sum_{i=1}^{n} a_{p, i} \boldsymbol{e}_{q, i}+\boldsymbol{e}_{p, q} \longleftarrow \boldsymbol{f} \\
& a_{p, q}-\delta_{p, q} \longleftarrow \boldsymbol{e}_{p, q} \quad-\sum_{j, k=1}^{n} a_{j, k} \boldsymbol{f}_{j, k}+\sum_{i=1}^{n} \boldsymbol{f}_{i, i} \longleftarrow \boldsymbol{f}
\end{aligned}
$$

Um die Dimension der Homologie bzw. Kohomologie zu berechnen betrachten wir die Auflösung von $\mathbb{K}_{\Lambda}$, ohne die erste nicht triviale Stelle unter dem Funktor $\left(\mathbb{K}_{\Omega} \otimes_{\mathcal{A}} \bullet\right)$ bzw. dem Funktor $\operatorname{HOM}_{\mathcal{A}}\left(\bullet, \mathbb{K}_{\Omega}\right)$. Mit anderen Worten, wir berechnen $\operatorname{Tor}^{\mathcal{A}}\left(\mathbb{K}_{\Omega}, \mathbb{K}_{\Lambda}\right)$ bzw. $\operatorname{Ext}_{\mathcal{A}}\left(\mathbb{K}_{\Omega}, \mathbb{K}_{\Lambda}\right)$. 


\subsection{Tor}

In diesem Kapitel wollen wir die Dimensionen der Homologie über die Tor-Gruppen berechnen.

\section{Satz 4.1.1 (Tor)}

Sei $\mathcal{H} \mathcal{H}_{i}$ die Homologie des eindimensionalen Moduls $\mathbb{K}_{\Lambda}$, wobei $i=1,2$, 3. Dann gelten für die Dimensionen folgende Werte:

\begin{tabular}{|c|c|c|c|}
\hline & $\Omega=\Lambda$ & $\Omega=-\Lambda$ & sonst \\
\hline $\operatorname{Dim}\left(\mathcal{H H}_{0}\right)$ & 1 & 0 & 0 \\
\hline $\operatorname{Dim}\left(\mathcal{H H}_{1}\right)$ & $\frac{n^{2}-n}{2}$ & $\frac{n^{2}+n-2}{2}$ & $k_{-1}+k_{\Lambda}-1$ \\
\hline $\operatorname{Dim}\left(\mathcal{H H}_{2}\right)$ & $\frac{n^{2}-n}{2}$ & $\frac{n^{2}+n-2}{2}$ & $k_{-1}+k_{\Lambda}-1$ \\
\hline $\operatorname{Dim}\left(\mathcal{H H}_{3}\right)$ & 1 & 0 & 0 \\
\hline
\end{tabular}

mit $k_{-1}:=\#\left\{i \mid r_{i}=-1\right\}$ und $k_{\Lambda}:=\#\left\{(i, j) \mid i>j, r_{i} r_{j}=1\right\}$, wobei $r_{i}$ die Vielfachheit der Nullstellen des Charakteristischenpolynoms von $\Omega \Lambda^{t}$ sind.

Den Komplex erhalten wir durch Anwenden des Funktors $\left(\mathbb{K}_{\Omega} \otimes_{\mathcal{A}} \bullet\right)$ auf die in 4.0.14 gegebene Auflösung. Sie sieht wegen $\mathbb{K}_{\Omega} \otimes_{\mathcal{A}} \mathcal{A} \cong \mathbb{K}$ wie folgt aus:

\begin{tabular}{|l|}
\hline \\
$0 \leftarrow \mathbb{K} \stackrel{\phi_{1 *}}{\longleftarrow} \mathbb{K}^{n^{2} \stackrel{\phi_{2 *}}{\longleftarrow}} \mathbb{K}^{n^{2} \stackrel{\phi_{3 *}}{\longleftarrow}} \mathbb{K} \leftarrow 0$ \\
$\phi_{1 *}: \boldsymbol{E} \mapsto(\Omega-\Lambda) \boldsymbol{e}$ \\
$\phi_{2 *}: \boldsymbol{F} \mapsto \Omega \boldsymbol{E}^{t}+\left(\Lambda \boldsymbol{E}^{t}\right)^{t}$ \\
$\phi_{3 *}: \boldsymbol{f} \mapsto-\operatorname{tr}\left(\Lambda \Omega^{t} \boldsymbol{F}\right)+\operatorname{tr}(\boldsymbol{F})$ \\
\hline
\end{tabular}

Die Homologie bekommen wir aus dem Komplex durch:

$$
\mathcal{H} \mathcal{H}_{i}:=\operatorname{Kern}\left(\Phi_{i *}\right) / \operatorname{Bild}\left(\Phi_{i+1 *}\right) .
$$

Wir berechnen die Dimensionen über:

$$
\operatorname{Dim}\left(\operatorname{Kern}\left(\Phi_{i *}\right) / \operatorname{Bild}\left(\Phi_{i+1 *}\right)\right)=\operatorname{Dim}\left(\operatorname{Kern}\left(\Phi_{i *}\right)\right)-\operatorname{Rang}\left(\Phi_{i+1 *}\right)
$$


4.1.1 $\Phi_{1 *}: E \mapsto \Omega e-\Lambda e$

\begin{tabular}{|c|c|c|c|}
\hline \multicolumn{2}{|c|}{$\operatorname{Dim}\left(\operatorname{Kern}\left(\Phi_{1 *}\right)\right)$} & \multicolumn{2}{|c|}{$\operatorname{Rang}\left(\Phi_{1 *}\right)$} \\
\hline$\Omega=\Lambda$ & sonst & $\Omega=\Lambda$ & sonst \\
\hline$n^{2}$ & $n^{2}-1$ & 0 & 1 \\
\hline
\end{tabular}

Sei zunächst $\Omega=\Lambda$, dann ist $\operatorname{Bild}\left(\Phi_{1 *}\right)=0$ und der $\operatorname{Kern}\left(\Phi_{1 *}\right)$ ist das gesamte Urbild, also ist $\operatorname{Rang}\left(\Phi_{1 *}\right)=0$ und $\operatorname{Dim}\left(\operatorname{Kern}\left(\Phi_{1 *}\right)\right)=n^{2}$.

Falls $\Omega \neq \Lambda$, dann existiert wenigstens ein $(p, q)$ mit $\omega_{p, q} \neq \lambda_{p, q}$. Dann ist $\Phi_{1 *}\left(e_{p, q}\right) \neq$ 0 , also gilt $\operatorname{Rang}\left(\Phi_{1 *}\right)>0$. Da der Bildraum nur eindimensional ist, gilt $\operatorname{Rang}\left(\Phi_{1 *}\right)=$ 1 und mit $\operatorname{Dim}\left(\operatorname{Kern}\left(\Phi_{1 *}\right)\right)=\operatorname{Dim}($ Urbild $)-\operatorname{Dim}($ Bild $)=n^{2}-1$. 
4.1.2 $\Phi_{2 *}: \boldsymbol{F} \mapsto \Omega \boldsymbol{E}^{t}+\left(\Lambda \boldsymbol{E}^{t}\right)^{t}$

\begin{tabular}{|c|c|c|c|c|c|}
\hline \multicolumn{3}{|c|}{$\operatorname{Dim}\left(\operatorname{Kern}\left(\Phi_{2 *}\right)\right)$} & \multicolumn{3}{|c|}{$\operatorname{Rang}\left(\Phi_{2 *}\right)$} \\
\hline$\Omega=\Lambda$ & $\Omega=-\Lambda$ & sonst & $\Omega=\Lambda$ & $\Omega=-\Lambda$ & sonst \\
\hline$\frac{n^{2}-n}{2}$ & $\frac{n^{2}+n}{2}$ & $k_{-1}+k_{\wedge}$ & $\frac{n^{2}+n}{2}$ & $\frac{n^{2}-n}{2}$ & $n^{2}-\left(k_{-1}+k_{\bigwedge}\right)$ \\
\hline
\end{tabular}

Mit $k_{-1}:=\#\left\{i \mid r_{i}=-1\right\}$ und $k_{\wedge}:=\#\left\{(i, j) \mid i>j, r_{i} r_{j}=1\right\}$, die Vielfachheit der Nullstellen des Charakteristischenpolynoms von $\Omega \Lambda^{t}$ sind.

Wir werden nun $\Phi_{2 *}$ als $n^{2} \times n^{2}$-Matrix schreiben. Da wir von links multiplizieren, steht in der Zeile $(p-1) n+q$ das Bild von $f_{p, q}$ bezüglich der Basis des Bildraumes:

$$
\Phi_{2 *}\left(f_{p, q}\right)=\sum_{i=1}^{n}\left(\omega_{p, i} e_{q, i}+\lambda_{q, i} e_{p, i}\right) .
$$

Um uns Schreibarbeit zu sparen schreiben wir $\lambda_{p}$. bzw. $\omega_{p}$, für den Zeilenvektor $\left(\lambda_{p, 1}, \lambda_{p, 2}, \ldots, \lambda_{p, n}\right)$ bzw. $\left(\omega_{p, 1}, \omega_{p, 2}, \ldots, \omega_{p, n}\right)$. Den Nullvektor schreiben wir als $0_{n}:=(\underbrace{0, \ldots, 0}_{n})$.

Die Matrix $\Lambda$ können wir dann schreiben als:

$$
\Lambda=\left(\begin{array}{c}
\lambda_{1, \cdot} \\
\lambda_{2, \cdot} \\
\vdots
\end{array}\right)
$$

Die Matrix $\Phi_{2 *}$ ist dann:

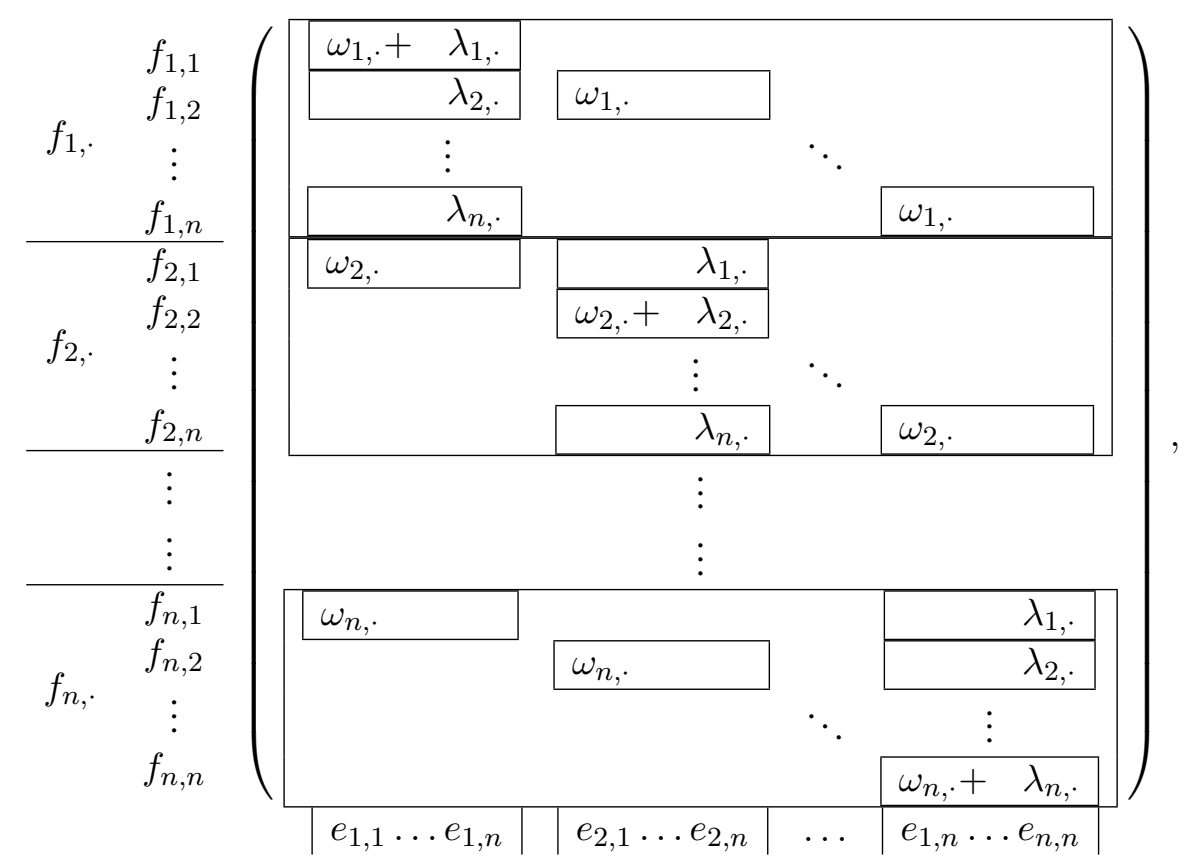


wobei die leeren Stellen jeweils mit Nullen gefüllt sein sollen. Alternativ lässt sich die Matrix schreiben als:

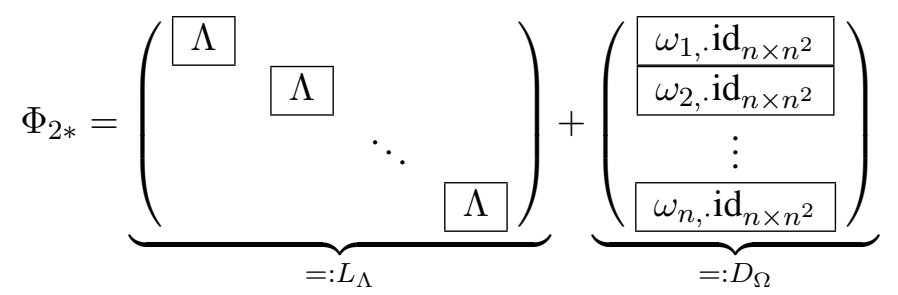

Da $\Lambda$ mit $\Lambda^{t}$ invertierbar, ist die Matrix $L_{\Lambda}$ mit $L_{\Lambda^{t}}$ invertierbar. Das Multiplizieren mit einer invertierbaren Matrix ändert den Rang einer Matrix nicht, daher können wir anstelle von $\Phi_{2 *}$ auch folgende Matrix betrachten:

$$
\Phi_{2 *} L_{\Lambda^{t}}=\mathrm{id}+D_{\Omega} L_{\Lambda^{t}}=\mathrm{id}+D_{\Omega \Lambda^{t}},
$$

da für das Produkt aus den Matrizen $D_{\Omega}$ und $L_{\Lambda^{t}}$ gilt:

$$
D_{\Omega} L_{\Lambda^{t}}=D_{\Omega \Lambda^{t}} .
$$

4.1.3 1. Fall: $\mathbb{K}=\mathbb{R}$ und $n=2$

Dann sind $\Lambda$ und $\Omega$ Drehmatrizen oder Spiegelungsmatrizen. Also gibt es ein $\psi$ mit:

$$
\Omega \Lambda^{t}=\left(\begin{array}{cc}
\cos \psi & -\sin \psi \\
\sin \psi & \cos \psi
\end{array}\right) \text { oder } \Omega \Lambda^{t}=\left(\begin{array}{cc}
\cos \psi & \sin \psi \\
\sin \psi & -\cos \psi
\end{array}\right) .
$$

Die Matrix id $+D_{\Omega \Lambda^{t}}$ ist dann:

$$
\left(\begin{array}{cccc}
\cos \psi+1 & -\sin \psi & 0 & 0 \\
0 & 1 & \cos \psi & -\sin \psi \\
\sin \psi & \cos \psi & 1 & 0 \\
0 & 0 & \sin \psi & \cos \psi+1
\end{array}\right) \operatorname{oder}\left(\begin{array}{cccc}
\cos \psi+1 & \sin \psi & 0 & 0 \\
0 & 1 & \cos \psi & \sin \psi \\
\sin \psi & -\cos \psi & 1 & 0 \\
0 & 0 & \sin \psi & -\cos \psi+1
\end{array}\right) \text {. }
$$

Für den Spezialfall $\cos \psi=-1$ vereinfacht sich die Matrix zu:

$$
\left(\begin{array}{cccc}
0 & 0 & 0 & 0 \\
0 & 1 & -1 & 0 \\
0 & -1 & 1 & 0 \\
0 & 0 & 0 & 0
\end{array}\right) \text { oder }\left(\begin{array}{cccc}
0 & 0 & 0 & 0 \\
0 & 1 & -1 & 0 \\
0 & 1 & 1 & 0 \\
0 & 0 & 0 & 2
\end{array}\right)
$$

Der Rang ist also 1 oder 3. Dieser Spezialfall (für $\Omega \Lambda^{t}$ ist eine Drehmatrix) tritt genau dann ein, wenn $\Lambda=-\Omega$.

Falls $\cos \psi \neq-1$, kann man die Matrizen mittels des Gaussalgorithmus in folgende Form bringen:

$$
\left(\begin{array}{cccc}
\sin \psi & -\frac{\sin ^{2} \psi}{\cos \psi+1} & 0 & 0 \\
0 & 1 & \cos \psi & -\sin \psi \\
0 & 0 & 0 & 0 \\
0 & 0 & \frac{\sin ^{2} \psi}{\cos \psi+1} & \sin \psi
\end{array}\right) \text { oder }\left(\begin{array}{cccc}
\sin \psi & \frac{\sin ^{2} \psi}{\cos \psi+1} & 0 & 0 \\
0 & 1 & \cos \psi & \sin \psi \\
0 & 0 & \sin \psi & \frac{\sin ^{2} \psi}{\cos \psi+1} \\
0 & 0 & 0 & 0
\end{array}\right)
$$

Der Rang ist also 3. 


\section{Fall $\Omega \Lambda^{t}$ ist eine Drehmatrix:}

$$
\left(\begin{array}{cccc}
\sin \psi & -\frac{\sin ^{2} \psi}{\cos \psi+1} & 0 & 0 \\
0 & 1 & \cos \psi & -\sin \psi \\
\sin \psi & \cos \psi & 1 & 0 \\
0 & 0 & \sin \psi & \cos \psi+1
\end{array}\right) \rightarrow\left(\begin{array}{cccc}
\sin \psi & -\frac{\sin ^{2} \psi}{\cos \psi+1} & 0 & 0 \\
0 & 1 & \cos \psi & -\sin \psi \\
0 & \cos \psi+\frac{\sin ^{2} \psi}{\cos \psi+1} & 1 & 0 \\
0 & 0 & \sin \psi & \cos \psi+1
\end{array}\right)
$$

mit $\cos \psi+\frac{\sin ^{2} \psi}{\cos \psi+1}=\frac{\cos ^{2} \psi+\cos \psi+\sin ^{2} \psi}{\cos \psi+1}=1$

$$
\begin{aligned}
\left(\begin{array}{cccc}
\sin \psi & -\frac{\sin ^{2} \psi}{\cos \psi+1} & 0 & 0 \\
0 & 1 & \cos \psi & -\sin \psi \\
0 & 1 & 1 & 0 \\
0 & 0 & \frac{\sin \psi}{\cos \psi+1} & 1
\end{array}\right) \rightarrow\left(\begin{array}{cccc}
\sin \psi & -\frac{\sin ^{2} \psi}{\cos \psi+1} & 0 & 0 \\
0 & 1 & \cos \psi & -\sin \psi \\
0 & 0 & 1-\cos \psi & \sin \psi \\
0 & 0 & \frac{\sin ^{2} \psi}{\cos \psi+1} & \sin \psi
\end{array}\right) \\
\operatorname{mit} \frac{\sin ^{2} \psi}{\cos \psi+1}-(1-\cos \psi)=\frac{\sin ^{2} \psi-\left(1-\cos ^{2} \psi\right)}{\cos \psi+1}=0 \\
\rightarrow\left(\begin{array}{cccc}
\sin \psi & -\frac{\sin ^{2} \psi}{\cos \psi+1} & 0 & 0 \\
0 & 1 & \cos \psi & -\sin \psi \\
0 & 0 & 0 & 0 \\
0 & 0 & \frac{\sin ^{2} \psi}{\cos \psi+1} & \sin \psi
\end{array}\right)
\end{aligned}
$$

\section{Fall $\Omega \Lambda^{t}$ ist eine Spiegelungsmatrix:}

$$
\begin{aligned}
& \left(\begin{array}{cccc}
\cos \psi+1 & \sin \psi & 0 & 0 \\
0 & 1 & \cos \psi & \sin \psi \\
\sin \psi & -\cos \psi & 1 & 0 \\
0 & 0 & \sin \psi & -\cos \psi+1
\end{array}\right) \rightarrow\left(\begin{array}{cccc}
\sin \psi & \frac{\sin ^{2} \psi}{\cos \psi+1} & 0 & 0 \\
0 & 1 & \cos \psi & \sin \psi \\
\sin \psi & -\cos \psi & 1 & 0 \\
0 & 0 & \sin \psi & -\cos \psi+1
\end{array}\right) \\
& \rightarrow\left(\begin{array}{cccc}
\sin \psi & \frac{\sin ^{2} \psi}{\cos \psi+1} & 0 & 0 \\
0 & 1 & \cos \psi & \sin \psi \\
0 & -\cos \psi-\frac{\sin ^{2} \psi}{\cos \psi+1} & 1 & 0 \\
0 & 0 & \sin \psi & -\cos \psi+1
\end{array}\right) \\
& \text { mit }-\cos \psi-\frac{\sin ^{2} \psi}{\cos \psi+1}=-\frac{\cos ^{2} \psi+\cos \psi+\sin ^{2} \psi}{\cos \psi+1}=-1 \\
& \rightarrow\left(\begin{array}{cccc}
\sin \psi & \frac{\sin ^{2} \psi}{\cos \psi+1} & 0 & 0 \\
0 & 1 & \cos \psi & \sin \psi \\
0 & -1 & 1 & 0 \\
0 & 0 & \sin \psi & -\cos \psi+1
\end{array}\right) \rightarrow\left(\begin{array}{cccc}
\sin \psi & \frac{\sin ^{2} \psi}{\cos \psi+1} & 0 & 0 \\
0 & 1 & \cos \psi & \sin \psi \\
0 & 0 & 1+\cos \psi & \sin \psi \\
0 & 0 & \sin \psi & -\cos \psi+1
\end{array}\right) \\
& \text { mit } \frac{\sin ^{2} \psi}{\cos \psi+1}-(1-\cos \psi)=\frac{\sin ^{2} \psi-\left(1-\cos ^{2} \psi\right)}{\cos \psi+1}=0 \\
& \rightarrow\left(\begin{array}{cccc}
\sin \psi & \frac{\sin ^{2} \psi}{\cos \psi+1} & 0 & 0 \\
0 & 1 & \cos \psi & \sin \psi \\
0 & 0 & \sin \psi & \frac{\sin ^{2} \psi}{\cos \psi+1} \\
0 & 0 & 0 & 0
\end{array}\right)
\end{aligned}
$$


4.1.4 2. Fall: $\Omega=\Lambda$, also $\Omega \Lambda^{t}=\mathrm{id}$

Die Matrix id $+D_{\text {id }}$ ist in diesem Fall:

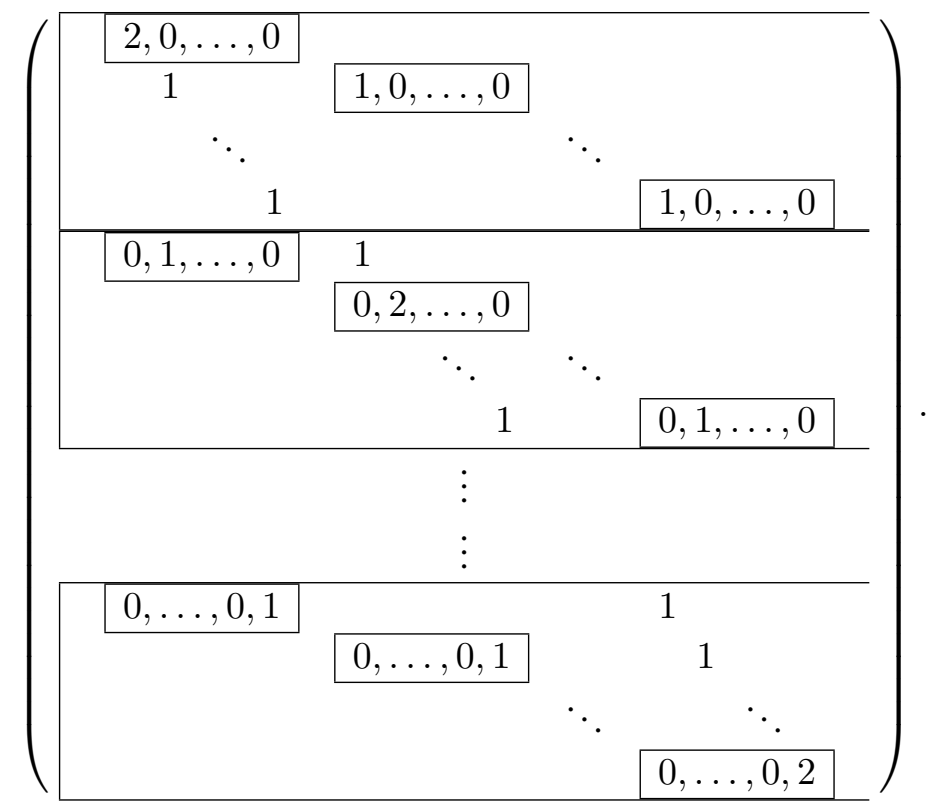

Die Zeilen zu den Basisvektoren $f_{p, q}$ und $f_{q, p}$ stimmen für jedes Paar $(p, q)$ überein. Alle anderen Zeilen sind linear unabhängig. Der Rang ist daher $\sum_{i=1}^{n} i=\frac{n^{2}+n}{2}$. 
4.1.5 3. Fall: $\Omega=-\Lambda$, also $\Omega \Lambda^{t}=-\mathrm{id}$

Die Matrix id $+D_{- \text {id }}$ ist in diesem Fall:

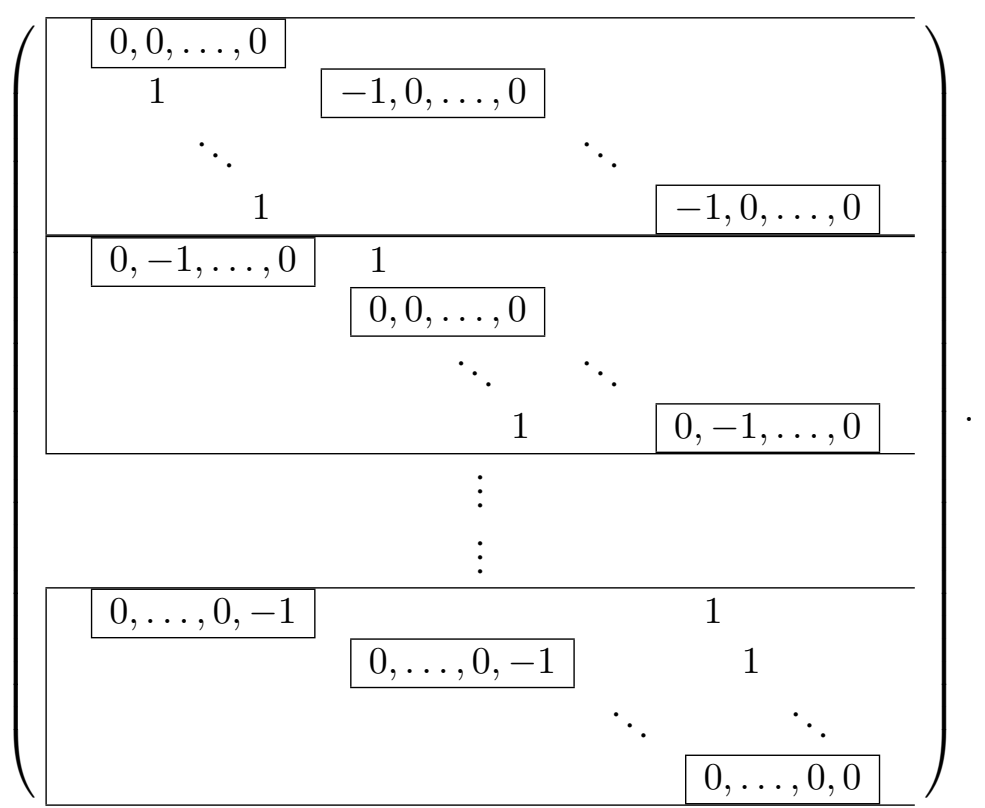

Die Zeilen zu den Basisvektoren $f_{i, i}$ bestehen für jedes $i$ nur aus Nullen. Die Zeilen zu den Basisvektoren $f_{p, q}$ und $f_{q, p}$ stimmen für jedes Paar $(p, q)$ bis auf das Vorzeichen überein. Alle anderen Zeilen sind linear unabhängig. Der Rang ist daher $\sum_{i=1}^{n} i-n=$ $\frac{n^{2}-n}{2}$. 


\subsubsection{Allgemeiner Fall}

In diesem Abschnitt wollen wir den Rang von id $+D_{\Omega \Lambda^{t}}$ für eine beliebige orthogonale Matrix $\Omega \Lambda^{t}$ berechnen.

Zuerst stellen wir fest, dass wir den Rang von id $+D_{\Omega \Lambda^{t}}$ über die Vielfachheit der Nullstellen des Charakteristischen Polynoms an der Stelle -1 berechnen können:

$$
\operatorname{Rang}\left(\Phi_{\mathrm{id}+D_{\Omega \Lambda^{t}}}\right)=n^{2}-\mathrm{NS}_{-1}\left(\operatorname{Det}\left(x \mathrm{id}-D_{\Omega \Lambda^{t}}\right)\right) .
$$

Wir werden nun das Charakteristische Polynom von $D_{\Psi}$ für eine beliebige orthogonale Matrix $\Psi$ berechnen.

Dazu wollen wir uns überlegen, warum wir annehmen können, dass die Matrix $\Psi$ in Jordanform vorliegt.

Falls $\mathbb{K}$ nicht algebraisch abgeschlossen ist, verändert sich das Charakteristische Polynom von $D_{\Psi}$ über einem Abschluss von $\mathbb{K}$ nicht. Wir können also ohne Beschränkung der Allgemeinheit annehmen, dass $\mathbb{K}$ algebraisch abgeschlossen ist.

Für eine Matrix $\Psi$ gibt es in einem algebraisch abgeschlossenen Körper $\mathbb{K}$ eine Jordanform. Es gibt also eine Matrix $N$ und eine Jordan-Matrix $J_{\Psi}$, so dass gilt: $\Psi N=N J_{\Psi}$.

\section{Lemma 4.1.2 (Konjugieren ist verträglich mit $D$ )}

Seien $M, N$ und $X$ quadratische Matrizen, die $M X=X N$ erfüllen. Dann gilt:

$$
D_{M}(X \otimes X)=(X \otimes X) D_{N} .
$$

Beweis: [Lemma 4.1.2]

Um Schreibarbeit zu sparen bezeichnen wir die $p$-te Zeile der Matrix $M$ bzw. $N$ mit

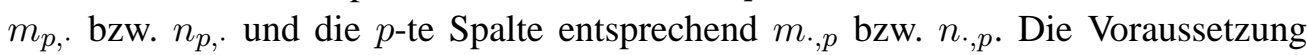
$M X=X N$ lässt sich dann schreiben als:

$$
\sum_{i=1}^{n} m_{p, i} x_{i, q}=\sum_{i=1}^{n} x_{p, i} n_{i, q} \quad \forall(p, q)
$$

oder alternativ:

$$
\left\langle m_{p, \cdot}, x_{\cdot, q}\right\rangle=\left\langle x_{p, \cdot}, n_{\cdot, q}\right\rangle \quad \forall(p, q) .
$$

Die Matrix $(X \otimes X)$ ist definiert durch:

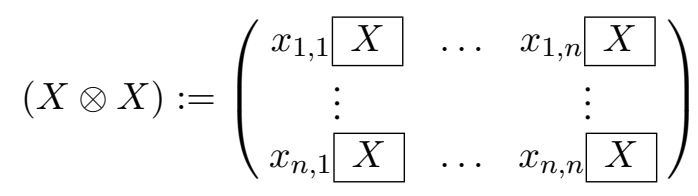

Für eine $n^{2} \times n^{2}$-Matrix $Y$ bezeichnen wir die Spalten und Zeilen wie zuvor nach den zugehörigen Basisvektoren. An der Stelle $((p, q),(i, j))$, also im $p$-ten Zeilenblock im $i$-ten Spaltenblock an der $(q, j)$-Stelle, steht der Anteil des $(i, j)$-ten Basisvektor des 
Bildes zum Basisvektor $e_{p, q}$.

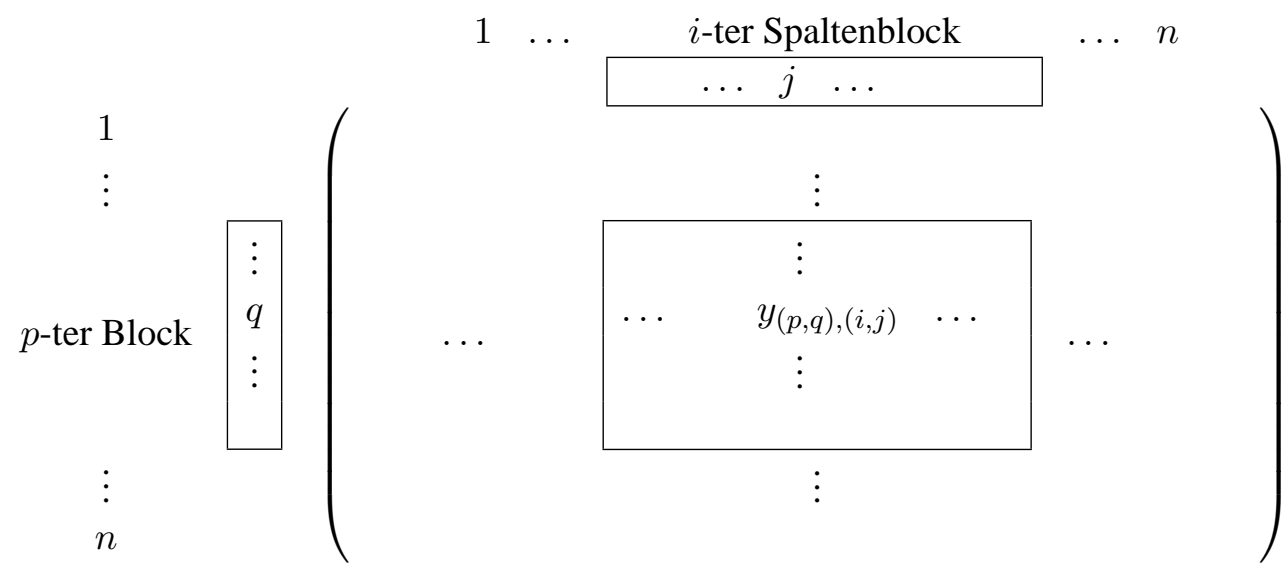

Da in jeder Zeile von $D_{M}$ nur ein $n$-Tupel steht, lässt sich das Produkt $D_{M}(X \otimes X)$ leicht berechnen:

$$
\left(D_{M}(X \otimes X)\right)_{(p, q),(i, j)}:=\left\langle m_{p, \cdot}, x_{i, q} x_{\cdot, j}\right\rangle \quad \forall((p, q),(i, j)) .
$$

In jeder Zeile von $D_{N}$ stehen wiederum nur wenige Einträge, nämlich ein Spaltenvektor auf verschiedene Blöcke verteilt. Daher gilt:

$$
\left((X \otimes X) D_{N}\right)_{(p, q),(i, j)}:=\left\langle x_{p, \cdot} x_{i, q}, m_{\cdot, j}\right\rangle \quad \forall((p, q),(i, j)) .
$$

Mit der Voraussetzung $\left\langle m_{p, \cdot}, x_{,, q}\right\rangle=\left\langle x_{p, \cdot}, n_{\cdot, q}\right\rangle \quad \forall(p, q)$ erhalten wir für jedes $(p, q),(i, j)$ :

$$
\begin{aligned}
\left((X \otimes X) D_{N}\right)_{(p, q),(i, j)}: & =\left\langle x_{p, \cdot} \cdot x_{i, q}, n_{\cdot, j}\right\rangle \\
& =x_{i, q}\left\langle x_{p, \cdot}, n \cdot, j\right\rangle \\
& =x_{i, q}\left\langle m_{p, \cdot}, x_{\cdot, j}\right\rangle \\
& =\left\langle m_{p, \cdot}, x_{i, q} x_{\cdot, j}\right\rangle \\
& =:\left(D_{M}(X \otimes X)\right)_{(p, q),(i, j)} .
\end{aligned}
$$

Als Folgerung daraus erhalten wir, dass das Charakteristische Polynom von $D_{\Psi}$ mit dem Charakteristischen Polynom von $D_{J_{\Psi}}$ übereinstimmt.

Satz 4.1.3 (Charakteristisches Polynom von $D_{J}$ )

Sei J eine Jordan-Matrix, dann gilt für das Charakteristische Polynom:

$$
\chi_{D_{J}}(x)=\chi_{J}(x) \cdot \chi_{\bigwedge^{2} J}\left(x^{2}\right) .
$$

Beweis: [Satz 4.1.3

Sei

$$
J:=\left(\begin{array}{cccc}
r_{1} & \epsilon_{1} & & \\
& \ddots & \ddots & \\
& & r_{n-1} & \epsilon_{n-1} \\
& & & r_{n}
\end{array}\right),
$$


wobei die leeren Stellen mit Nullen gefüllt sein sollen. Dann ist die Matrix $D_{J}$ ein Endomorphismus auf einem $n^{2}$-dimensionalen Vektorraum. Sie ist von folgender Form:

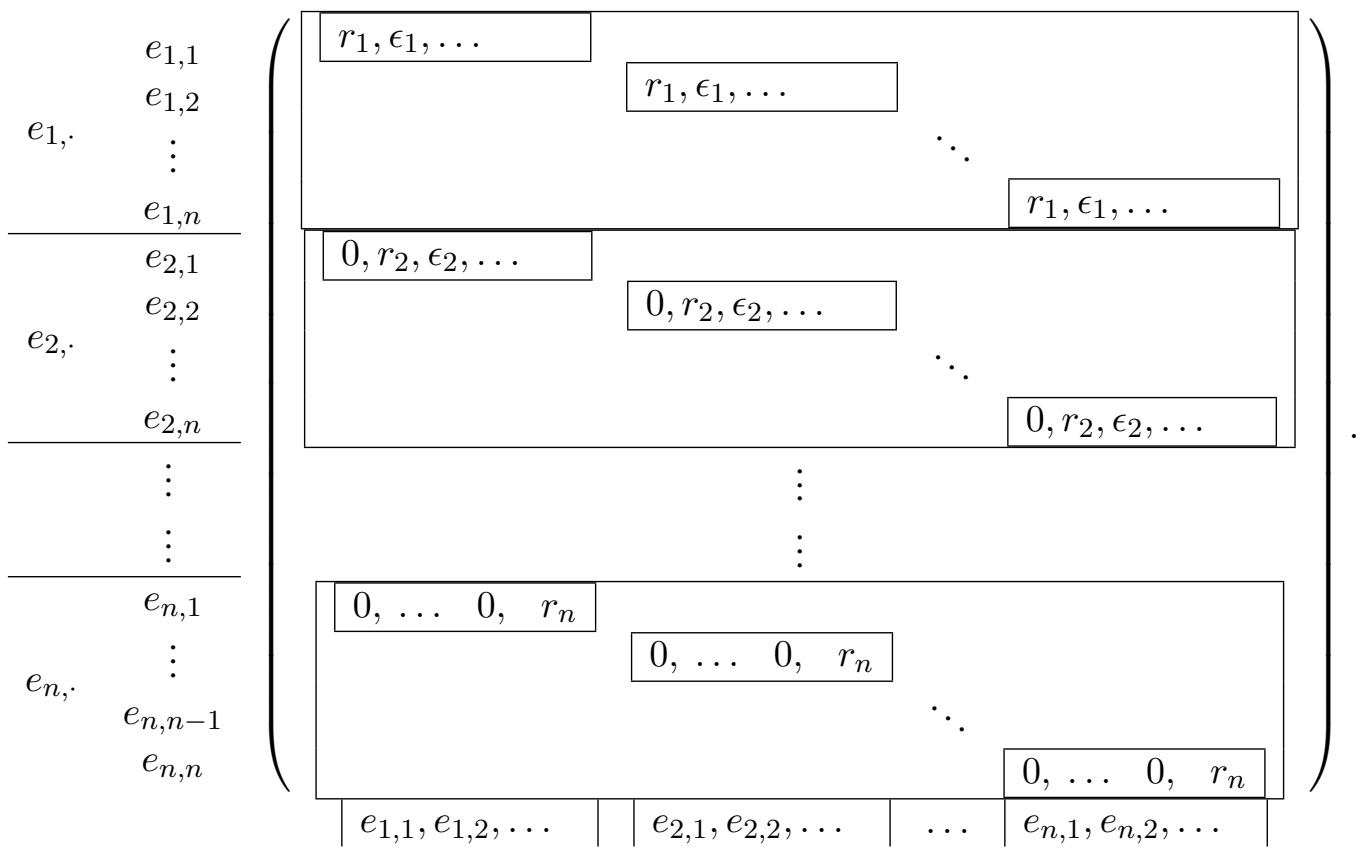

In den Beispielen 4.1.4 und 4.1.5 haben wir gesehen, dass die Zeilen zu den Basisvektoren $e_{p, p}, e_{p, q}$ und $e_{q, p}$ eine besondere Rolle spielen. Daher sortieren wir die Basisvektoren $e_{p, q}$ nach der Summe aus $p$ und $q$ um. Falls die Summe gleich ist, sortieren wir nach dem zweiten Index. Auf diese Weise folgen die Paare $e_{p, q}$ und $e_{q, p}$ aufeinander, gefolgt von $e_{p, p}$.

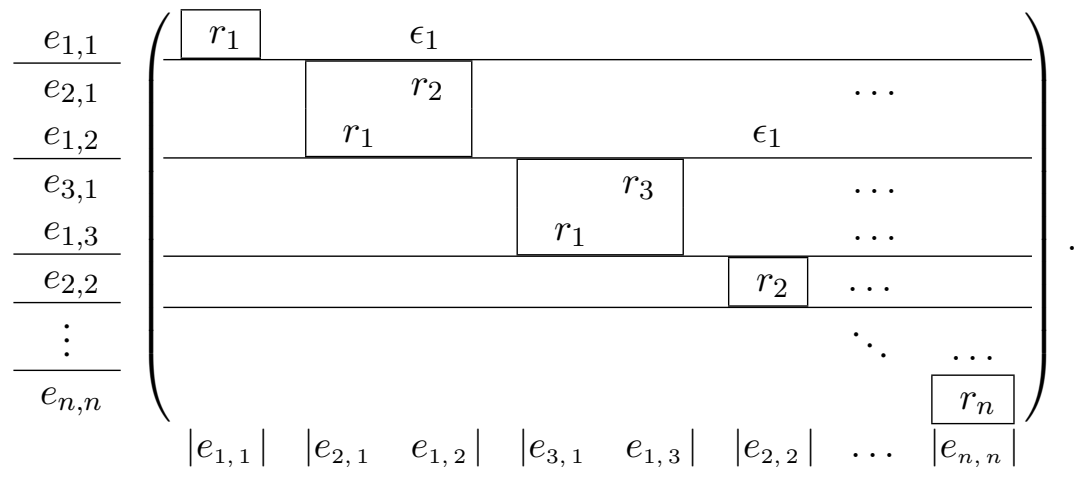

In der Zeile zum Basisvektor $e_{p, q}$ kommen nur $r_{p}$ und $\epsilon_{p}$ vor, wobei $\epsilon_{p}$ weiter links steht. Die Matrix $D_{J}$ sieht fast wie eine obere Dreiecksmatrix aus. Auf der Diagonalen stehen $1 \times 1$-und $2 \times 2$-Blöcke und in der oberen rechten Ecke steht an einigen Stellen ein $\epsilon$. 
Das Charakteristische Polynom berechnen wir über $\operatorname{Det}\left(x \mathrm{id}-D_{J}\right)$, also:

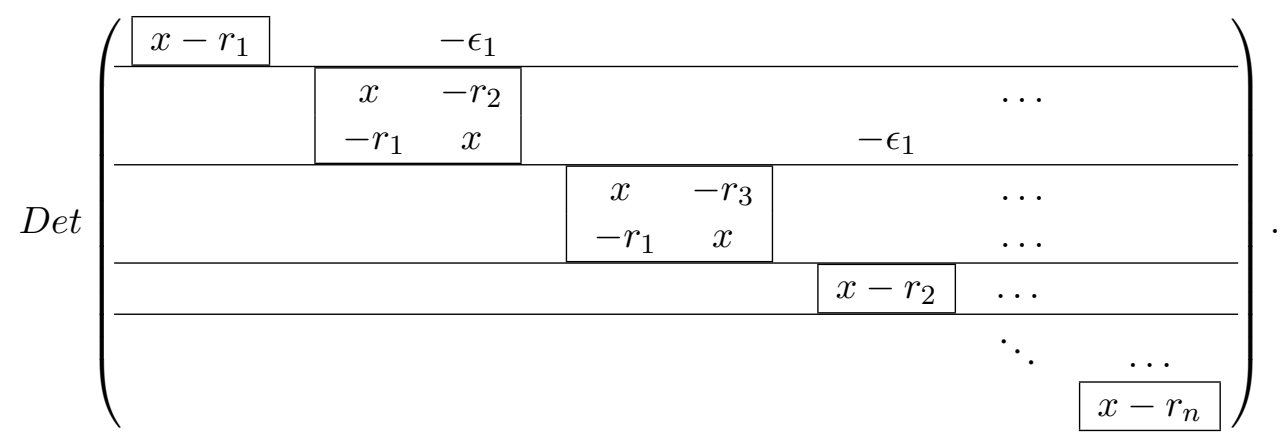

Für die Determinante von Matrizen, die sich in Blockmatrizen zerlegen lassen, wobei der untere Block leer ist, ist die Determinante gleich dem Produkt der Determinanten auf der Diagonalen:

$\operatorname{Det}\left(x \mathrm{id}-D_{J}\right)=\left(x-r_{1}\right) \operatorname{Det}\left(\begin{array}{cc}x & -r_{2} \\ -r_{1} & x\end{array}\right) \operatorname{Det}\left(\begin{array}{cc}x & -r_{3} \\ -r_{1} & x\end{array}\right)\left(x-r_{2}\right) \cdots\left(x-r_{n}\right)$.

$\operatorname{Da} \operatorname{Det}\left(\begin{array}{cc}x & -r_{p} \\ -r_{q} & x\end{array}\right)=x^{2}-r_{p} r_{q}$, können wir das Charakteristische Polynom von $D_{J}$ schreiben als:

$$
\chi_{D_{J}}(x)=\left(\prod_{i=1}\left(x-r_{i}\right)\right)\left(\prod_{i>j}\left(x^{2}-r_{i} r_{j}\right)\right) .
$$

Dies entspricht gerade der Behauptung: $\chi_{D_{J}}(x)=\chi_{J}(x) \cdot \chi_{\Lambda^{2} J}\left(x^{2}\right)$.

Zusammen mit den Vorüberlegungen erhalten wir, dass Rang $\left(\Phi_{\Phi_{2 *}}\right)$ nur von Eigenwerten $r_{i}$ der Matrix $\Omega \Lambda^{t}$ abhängt. Sei dazu

$$
\begin{aligned}
k_{-1} & :=\#\left\{i \mid r_{i}=-1\right\} \\
k_{\wedge} & :=\#\left\{(i, j) \mid i>j, r_{i} r_{j}=1\right\} .
\end{aligned}
$$

Der Rang ist dann:

$$
\operatorname{Rang}\left(\Phi_{\Phi_{2 *}}\right)=n^{2}-\left(k_{-1}+k_{\bigwedge}\right)
$$

und der Kern:

$$
\operatorname{Kern}\left(\Phi_{2 *}\right)=\left(k_{-1}+k_{\bigwedge}\right) .
$$




\subsection{7 $\Phi_{3 *}: \boldsymbol{f} \mapsto-\operatorname{tr}\left(\Lambda \Omega^{t} \boldsymbol{F}\right)+\operatorname{tr}(\boldsymbol{F})$}

\begin{tabular}{|c|c|c|c|}
\hline \multicolumn{2}{|c|}{$\operatorname{Dim}\left(\operatorname{Kern}\left(\Phi_{3 *}\right)\right)$} & \multicolumn{2}{|c|}{$\operatorname{Rang}\left(\Phi_{3 *}\right)$} \\
\hline$\Omega=\Lambda$ & sonst & $\Omega=\Lambda$ & sonst \\
\hline 1 & 0 & 0 & 1 \\
\hline
\end{tabular}

Sei zunächst $\Omega=\Lambda$, dann ist $\Lambda^{t} \Omega=$ id und der $\operatorname{Kern}\left(\Phi_{3 *}\right)$ das gesamte Urbild. Da der Urbildraum eindimensional ist, gilt Rang $\left(\Phi_{3 *}\right)=0$ und $\operatorname{Dim}\left(\operatorname{Kern}\left(\Phi_{3 *}\right)\right)=1$.

Falls $\Omega \neq \Lambda$, dann existiert wenigstens ein $(p, q)$ mit $\sum_{i=1}^{n} \lambda_{q, i} \omega_{p, i}+\delta_{p, q} \neq 0$; also gilt $\operatorname{Rang}\left(\Phi_{3 *}\right)>0$. Da der Urbildraum nur eindimensional ist, gilt $\operatorname{Rang}\left(\Phi_{3 *}\right)=1$ und mit $\operatorname{Dim}\left(\operatorname{Kern}\left(\Phi_{1 *}\right)\right)=\operatorname{Dim}($ Urbild $)-\operatorname{Dim}($ Bild $)=1-1=0$.

\subsection{Ext}

Die Extgruppen sind isomorph zu den Torgruppen. Hier erhält man die selben Matrizen, nur transponiert.

Wir betrachten, ähnlich wie in Kapitel 4.1, die induzierte, an der ersten Stelle nicht exakte, Sequenz:

$$
0 \leftarrow \boldsymbol{A} \stackrel{\phi_{1}}{\leftarrow} \boldsymbol{A}^{n^{2}} \stackrel{\phi_{2}}{\longleftarrow} \boldsymbol{A}^{n^{2}} \stackrel{\phi_{3}}{\leftarrow} \boldsymbol{A} \leftarrow 0
$$

Wir untersuchen zunächst den Vektorraum Isomorphismus $\operatorname{HOM}_{A}\left(A^{n}, \mathbb{K}_{\Omega}\right) \cong \mathbb{K}^{n}$. Sei $\boldsymbol{e}_{1}, \ldots, \boldsymbol{e}_{n}$ eine Basis des Moduls $\boldsymbol{A}^{n}$, dann ist $\overline{\boldsymbol{e}}_{1}, \ldots, \overline{\boldsymbol{e}}_{1}$ eine Basis von $\operatorname{HOM}_{\boldsymbol{A}}\left(\boldsymbol{A}^{n}, \mathbb{K}_{\Omega}\right)$ mit $\overline{\boldsymbol{e}}_{j}\left(\boldsymbol{e}_{i}\right)=\delta_{i, j}$. Lineares Fortsetzen liefert für $a_{i} \in \boldsymbol{A}$ :

$$
\overline{\boldsymbol{e}}_{j}\left(\sum_{i=1}^{n} a_{i} \boldsymbol{e}_{i}\right)=a_{j} .
$$

So erhalten wir aus der induzierten Sequenz unter dem kontravarianten Funktor $\operatorname{HOM}_{\boldsymbol{A}}\left(\cdot, \mathbb{K}_{\Omega}\right)$ :

$$
0 \rightarrow \mathbb{K}_{\Omega} \stackrel{\phi_{1}^{*}}{\rightarrow} \mathbb{K}^{n^{2}} \stackrel{\phi_{2}^{*}}{\rightarrow} \mathbb{K}^{n^{2}} \stackrel{\phi_{3}^{*}}{\rightarrow} \mathbb{K} \rightarrow 0
$$

Sei $\bar{e}$ der Basisvektor von $\mathbb{K}_{\Omega}$, dann sind die Abbildungen folgendermaßen definiert:

- $\phi_{1}^{*}: \overline{\boldsymbol{e}} \mapsto(\Omega-\Lambda) \overline{\boldsymbol{E}}$, wobei $\overline{\boldsymbol{E}}:=\left(\overline{\boldsymbol{e}}_{i, j}\right)$ die Matrix der Basisvektoren des Bildraumes ist. Die Abbildung erhalten wir durch:

$$
\overline{\boldsymbol{e}} \mapsto\left(\boldsymbol{e}_{p, q} \stackrel{\Phi_{1}}{\mapsto}\left(a_{p, q}-\lambda_{p, q}\right) \boldsymbol{e} \stackrel{\bar{e}}{\mapsto} \omega_{p, q}-\lambda_{p, q}\right)
$$

Diese Abbildung stimmt mit $\phi_{1 *}$ überein. 
- $\phi_{2}^{*}: \overline{\boldsymbol{E}} \mapsto\left(\Omega^{t} \overline{\boldsymbol{F}}\right)^{t}+\left(\Lambda^{t} \overline{\boldsymbol{F}}^{t}\right)^{t}$, wobei $\overline{\boldsymbol{F}}:=\left(\overline{\boldsymbol{f}}_{i, j}\right)$ die Matrix der Basisvektoren des Bildraumes ist. Die Abbildung erhalten wir durch:

$$
\overline{\boldsymbol{e}}_{p, q} \mapsto\left(\boldsymbol{f}_{x, y} \stackrel{\Phi_{2}}{\mapsto} \sum_{i=1}^{n}\left(a_{x, i} \boldsymbol{e}_{y, i}+\lambda_{y, i} \boldsymbol{e}_{x, i}\right) \stackrel{\bar{e}_{p, q}}{\sum_{i=1}^{n}\left(\omega_{x, i} \delta_{p, y} \delta_{q, i}+\lambda_{y, i} \delta_{p, x} \delta_{q, i}\right)}\right)
$$

also:

$$
\overline{\boldsymbol{e}}_{p, q} \mapsto \sum_{x, y=1}^{n}\left(\omega_{x, q} \delta_{p, y}+\lambda_{y, q} \delta_{p, x}\right) \overline{\boldsymbol{f}}_{x, y}=\sum_{x=1}^{n} \omega_{x, q} \overline{\boldsymbol{f}}_{x, p}+\sum_{y=1}^{n} \lambda_{y, q} \overline{\boldsymbol{f}}_{p, y}
$$

- $\phi_{3}^{*}: \overline{\boldsymbol{F}} \mapsto\left(\left(\Lambda \Omega^{t}\right)^{t}+\mathrm{id}\right) \overline{\boldsymbol{f}}$, wobei $\overline{\boldsymbol{f}}$ der Basisvektor des Bildraumes ist. Die Abbildung erhalten wir durch:

$$
\overline{\boldsymbol{f}}_{p, q} \mapsto\left(\boldsymbol{f} \stackrel{\Phi_{3}}{\mapsto} \sum_{i, j, k=1}^{n} \lambda_{k, i} a_{j, i} \boldsymbol{f}_{j, k}+\sum_{i=1}^{n} \boldsymbol{f}_{i, i} \stackrel{\overline{\boldsymbol{f}}_{p, q}}{\underbrace{\sum_{i, j, k=1}^{n} \lambda_{k, i} \omega_{j, i} \delta_{p, j} \delta_{q, k}+\sum_{i=1}^{n} \delta_{p, i} \delta_{q, i}}}\right)
$$

also:

$$
\overline{\boldsymbol{f}}_{p, q} \mapsto\left(\sum_{i=1}^{n} \lambda_{q, i} \omega_{p, i}+\delta_{p, q}\right) \overline{\boldsymbol{f}}
$$

Betrachten wir als Nächstes den Rang dieser Abbildungen.

\subsection{1 $\phi_{1}^{*}: \overline{\boldsymbol{e}} \mapsto(\Omega-\Lambda) \overline{\boldsymbol{E}}$}

Die Abbildung $\phi_{1}^{*}$ hat höchstens Rang 1, da der Urbildraum eindimensional ist. Sie hat Rang 0, wenn das Bild nur die 0 ist. Dies ist der Fall wenn:

$$
\Omega-\Lambda=0,
$$

also gilt:

$$
\operatorname{Rang}\left(\Phi_{1}^{*}\right)=\left\{\begin{array}{l}
0 \text { für } \Omega=\Lambda \\
1 \text { sonst }
\end{array}\right.
$$


4.2.2 $\phi_{2}^{*}: \overline{\boldsymbol{E}} \mapsto\left(\Omega^{t} \overline{\boldsymbol{F}}\right)^{t}+\left(\Lambda^{t} \overline{\boldsymbol{F}}^{t}\right)^{t}$

Hier beobachten wir, dass $\phi_{2}^{*}=\phi_{2 *}^{t}$ :

$$
\phi_{2}^{*}: \overline{\boldsymbol{E}} \mapsto\left(\Omega^{t} \overline{\boldsymbol{F}}\right)^{t}+\left(\Lambda^{t} \overline{\boldsymbol{F}}^{t}\right)^{t}=\left(\Omega^{t} \overline{\boldsymbol{F}}\right)^{t}+\left(\Lambda \overline{\boldsymbol{F}}^{t}\right)^{t}=\phi_{2 *}^{t}
$$

Wir betrachten die Abbildung in Matrizenschreibweise, wozu wir das Bild des Basisvektors $\overline{\boldsymbol{e}}_{p, q}$ in der Basis $\overline{\boldsymbol{f}}_{x, y}$ untersuchen:

$$
\begin{aligned}
\overline{\boldsymbol{e}}_{(p, q)}(x, y) & =\delta_{p, y} \omega_{x, q}+\delta_{p, x} \lambda_{y, q} \\
& =\sum_{p=1}^{n} \sum_{q=1}^{n}\left(\delta_{p, y} \omega_{x, q}+\delta_{p, x} \lambda_{y, q}\right) \boldsymbol{e}_{p, q} \\
& =\sum_{q=1}^{n} \omega_{x, y} \boldsymbol{e}_{y, q}+\sum_{p=1}^{n} \lambda_{y, q} \boldsymbol{e}_{x, q} \\
& =\phi_{2 *} .
\end{aligned}
$$

Also gilt :

$$
\operatorname{Rang}\left(\Phi_{2 *}\right)=\operatorname{Rang}\left(\Phi_{2}^{*}\right)
$$

4.2.3 $\phi_{3}^{*}: \overline{\boldsymbol{F}} \mapsto\left(\left(\Lambda \Omega^{t}\right)^{t}+\mathrm{id}\right) \overline{\boldsymbol{f}}$

Die Abbildung $\phi_{3}^{*}$ hat höchstens Rang 1, da der Bildraum eindimensional ist. Sie hat Rang 0, wenn das Bild nur die 0 ist. Dies ist nur dann der Fall, wenn:

$$
\left(\Lambda \Omega^{t}\right)^{t}=-\mathrm{id}
$$

also gilt:

$$
\operatorname{Rang}\left(\Phi_{1}^{*}\right)=\left\{\begin{array}{l}
0 \text { für } \Omega=\Lambda \\
1 \text { sonst }
\end{array} .\right.
$$




\section{Literaturverzeichnis}

[AL94] William W. Adams and Philippe Loustaunau. An introduction to Gröbner bases, volume 3 of Graduate Studies in Mathematics. American Mathematical Society, Providence, RI, 1994.

[Art91] Michael Artin. Algebra. Prentice Hall Inc., Englewood Cliffs, NJ, 1991.

[Ave95] Jürgen Avenhaus. Reduktionssysteme : Rechnen und Schließen in gleichungsdefinierten Strukturen. Springer-Lehrbuch. Springer-Verlag, Berlin [u.a.], 1995.

[BC07a] Teodor Banica and Benoît Collins. Integration over compact quantum groups. Publ. Res. Inst. Math. Sci., 43(2):277-302, 2007.

[BC07b] Teodor Banica and Benoît Collins. Integration over quantum permutation groups. J. Funct. Anal., 242(2):641-657, 2007.

[BCP97] Wieb Bosma, John Cannon, and Catherine Playoust. The Magma algebra system I: The user language. J. Symbolic Comput., 24(3-4):235-265, 1997. Computational algebra and number theory (London, 1993).

[Ber78a] George M. Bergman. The diamond lemma for ring theory. Adv. in Math., 29(2):178-218, 1978.

[Ber78b] George M. Bergman. The diamond lemma for ring theory. Adv. in Math., 29(2):178-218, 1978.

[BG94] Leo Bachmair and Harald Ganzinger. Buchberger's algorithm: a constraintbased completion procedure. In Constraints in computational logics (Munich, 1994), volume 845 of Lecture Notes in Comput. Sci., pages 285-301. Springer, Berlin, 1994.

[BO84] G. Bauer and F. Otto. Finite complete rewriting systems and the complexity of the word problem. Acta Inform., 21(5):521-540, 1984.

[Buc76] B. Buchberger. A theoretical basis for the reduction of polynomials to canonical forms. ACM SIGSAM Bull., 10(3):19-29, 1976.

[Buc01] Bruno Buchberger. Gröbner bases and systems theory. Multidimens. Systems Signal Process., 12(3-4):223-251, 2001. Special issue: Applications of Gröbner bases to multidimensional systems and signal processing. 
[Buc06] Bruno Buchberger. An algorithm for finding the basis elements of the residue class ring of a zero dimensional polynomial ideal. J. Symbolic Comput., 41(3-4):475-511, 2006. Translated from the 1965 German original by Michael P. Abramson.

[BW93] Thomas Becker and Volker Weispfenning. Gröbner bases, volume 141 of Graduate Texts in Mathematics. Springer-Verlag, New York, 1993. A computational approach to commutative algebra, in cooperation with Heinz Kredel.

[CLO92] David Cox, John Little, and Donal O'Shea. Ideals, varieties, and algorithms. Undergraduate Texts in Mathematics. Springer-Verlag, New York, 1992. An introduction to computational algebraic geometry and commutative algebra.

[Con85] Alain Connes. Noncommutative differential geometry. Inst. Hautes Études Sci. Publ. Math., (62):257-360, 1985.

[Deh11] M. Dehn. Über unendliche diskontinuierliche Gruppen. Math. Ann., 71(1):116-144, 1911.

[Der89] Nachum Dershowitz. Completion and its applications. In Resolution of equations in algebraic structures, Vol. 2, pages 31-85. Academic Press, Boston, MA, 1989.

[DKM90] Jeremy Dick, John Kalmus, and Ursula Martin. Automating the KnuthBendix ordering. Acta Inform., 28(2):95-119, 1990.

[Dri86] V. G. Drinfel'd. Quantum groups. Zap. Nauchn. Sem. Leningrad. Otdel. Mat. Inst. Steklov. (LOMI), 155(Differentsialnaya Geometriya, Gruppy Li i Mekh. VIII):18-49, 193, 1986.

[Eva51] Trevor Evans. The word problem for abstract algebras. J. London Math. Soc., 26:64-71, 1951.

[FFG93] Daniel R. Farkas, C. D. Feustel, and Edward L. Green. Synergy in the theories of Gröbner bases and path algebras. Canad. J. Math., 45(4):727$739,1993$.

[GAP06] The GAP Group. GAP - Groups, Algorithms, and Programming, Version 4.4.9, 2006.

[Gil79] Robert H. Gilman. Presentations of groups and monoids. J. Algebra, 57(2):544-554, 1979.

[GLS03] G.-M. Greuel, V. Levandovskyy, and H. Schönemann. SinguLAR::PluRAL 2.1. A Computer Algebra System for Noncommutative Polynomial Algebras, Centre for Computer Algebra, University of Kaiserslautern, 2003. http://www. singular.uni-kl.de/plural. 
[GPS05] G.-M. Greuel, G. Pfister, and H. Schönemann. Singular 3.0. A Computer Algebra System for Polynomial Computations, Centre for Computer Algebra, University of Kaiserslautern, 2005. http://www.singular.uni-kl.de.

[Gre99] Edward L. Green. Noncommutative Gröbner bases, and projective resolutions. In Computational methods for representations of groups and algebras (Essen, 1997), volume 173 of Progr. Math., pages 29-60. Birkhäuser, Basel, 1999.

[Gre03] David J. Green. Gröbner bases and the computation of group cohomology, volume 1828 of Lecture Notes in Mathematics. Springer-Verlag, Berlin, 2003.

[Hir64] Heisuke Hironaka. Resolution of singularities of an algebraic variety over a field of characteristic zero. I, II. Ann. of Math. (2) 79 (1964), 109-203; ibid. (2), 79:205-326, 1964.

[Jim85] Michio Jimbo. A $q$-difference analogue of $U(g)$ and the Yang-Baxter equation. Lett. Math. Phys., 10(1):63-69, 1985.

[Kac63] G. I. Kac. Ring groups and the duality principle. Trudy Moskov. Mat. Obšč., 12:259-301, 1963.

[KB70] Donald E. Knuth and Peter B. Bendix. Simple word problems in universal algebras. In Computational Problems in Abstract Algebra (Proc. Conf., Oxford, 1967), pages 263-297. Pergamon, Oxford, 1970.

[Lüc02] Wolfgang Lück. $L^{2}$-invariants: theory and applications to geometry and $K$-theory, volume 44 of Ergebnisse der Mathematik und ihrer Grenzgebiete. 3. Folge. A Series of Modern Surveys in Mathematics [Results in Mathematics and Related Areas. 3rd Series. A Series of Modern Surveys in Mathematics]. Springer-Verlag, Berlin, 2002.

[New42] M. H. A. Newman. On theories with a combinatorial definition of "equivalence.". Ann. of Math. (2), 43:223-243, 1942.

[Pie82] Richard S. Pierce. Associative algebras, volume 88 of Graduate Texts in Mathematics. Springer-Verlag, New York, 1982. , Studies in the History of Modern Science, 9.

[Ros87] Marc Rosso. Comparaison des groupes SU(2) quantiques de Drinfel'd et de Woronowicz. C. R. Acad. Sci. Paris Sér. I Math., 304(12):323-326, 1987.

[Rud91] Walter Rudin. Functional analysis. International Series in Pure and Applied Mathematics. McGraw-Hill Inc., New York, second edition, 1991.

[TC06] Andreas Thom and Benoit Collins. Homology of Free Quantum Groups. preprint, 2006. 
[Ufn89] V. A. Ufnarovskil. On the use of graphs for calculating the basis, growth and Hilbert series of associative algebras. Mat. Sb., 180(11):1548-1560, 1584, 1989.

[VDW96] Alfons Van Daele and Shuzhou Wang. Universal quantum groups. Internat. J. Math., 7(2):255-263, 1996.

[Wan95] Shuzhou Wang. Free products of compact quantum groups. Comm. Math. Phys., 167(3):671-692, 1995.

[Wor87] S. L. Woronowicz. Twisted SU(2) group. An example of a noncommutative differential calculus. Publ. Res. Inst. Math. Sci., 23(1):117-181, 1987.

[Wor88] S. L. Woronowicz. Tannaka-Kren̆n duality for compact matrix pseudogroups. Twisted $\mathrm{SU}(N)$ groups. Invent. Math., 93(1):35-76, 1988. 